

\section{Algebra \& Number Theory}

msp.berkeley.edu/ant

\section{EDITORS}

MANAGING EDITOR

Bjorn Poonen

Massachusetts Institute of Technology

Cambridge, USA

\author{
EDITORIAL BOARD CHAIR \\ David Eisenbud \\ University of California \\ Berkeley, USA
}

\section{BOARD OF EDITORS}

Georgia Benkart

Dave Benson

Richard E. Borcherds

John H. Coates

J-L. Colliot-Thélène

Brian D. Conrad

Hélène Esnault

Hubert Flenner

Edward Frenkel

Andrew Granville

Joseph Gubeladze

Ehud Hrushovski

Craig Huneke

Mikhail Kapranov

Yujiro Kawamata

János Kollár

Yuri Manin

Barry Mazur

Philippe Michel

Susan Montgomery
University of Wisconsin, Madison, USA

University of Aberdeen, Scotland

University of California, Berkeley, USA

University of Cambridge, UK

CNRS, Université Paris-Sud, France

University of Michigan, USA

Universität Duisburg-Essen, Germany

Ruhr-Universität, Germany

University of California, Berkeley, USA

Université de Montréal, Canada

San Francisco State University, USA

Hebrew University, Israel

University of Kansas, USA

Yale University, USA

University of Tokyo, Japan

Princeton University, USA

Northwestern University, USA

Harvard University, USA

École Polytechnique Fédérale de Lausanne

University of Southern California, USA
Shigefumi Mori

Raman Parimala

Jonathan Pila

Victor Reiner

Karl Rubin

Peter Sarnak

Joseph H. Silverman

Michael Singer

Ronald Solomon

Vasudevan Srinivas

J. Toby Stafford

Bernd Sturmfels

Richard Taylor

Ravi Vakil

Michel van den Bergh

Marie-France Vignéras

Kei-Ichi Watanabe

Andrei Zelevinsky

Efim Zelmanov
RIMS, Kyoto University, Japan

Emory University, USA

University of Oxford, UK

University of Minnesota, USA

University of California, Irvine, USA

Princeton University, USA

Brown University, USA

North Carolina State University, USA

Ohio State University, USA

Tata Inst. of Fund. Research, India

University of Michigan, USA

University of California, Berkeley, USA

Harvard University, USA

Stanford University, USA

Hasselt University, Belgium

Université Paris VII, France

Nihon University, Japan

Northeastern University, USA

University of California, San Diego, USA

\section{PRODUCTION}

contact@msp.org

Silvio Levy, Scientific Editor

See inside back cover or www.jant.org for submission instructions.

The subscription price for 2011 is US \$150/year for the electronic version, and \$210/year (+\$35 shipping outside the US) for print and electronic. Subscriptions, requests for back issues from the last three years and changes of subscribers address should be sent to Mathematical Sciences Publishers, Department of Mathematics, University of California, Berkeley, CA 94720-3840, USA.

Algebra \& Number Theory (ISSN 1937-0652) at Mathematical Sciences Publishers, Department of Mathematics, University of California, Berkeley, CA 94720-3840 is published continuously online. Periodical rate postage paid at Berkeley, CA 94704, and additional mailing offices.

ANT peer review and production are managed by EditFLOW ${ }^{\circledR}$ from Mathematical Sciences Publishers.

PUBLISHED BY

mathematical sciences publishers

http://msp.org/

A NON-PROFIT CORPORATION

Typeset in IAT $_{\mathrm{E}} \mathrm{X}$

Copyright @2011 by Mathematical Sciences Publishers 


\title{
Higher direct images of the structure sheaf in positive characteristic
}

\author{
Andre Chatzistamatiou and Kay Rülling
}

\begin{abstract}
We prove vanishing of the higher direct images of the structure (and the canonical) sheaf for a proper birational morphism with source a smooth variety and target the quotient of a smooth variety by a finite group of order prime to the characteristic of the ground field. We also show that for smooth projective varieties the cohomology of the structure sheaf is a birational invariant. These results are well known in characteristic zero.
\end{abstract}

Introduction

1. Chow groups with support 697

2. Hodge cohomology with support 718

3. Cycle class map to Hodge cohomology and applications 741

4. Generalization to tame quotients 752

$\begin{array}{ll}\text { Appendix } & 764\end{array}$

Acknowledgments $\quad 774$

$\begin{array}{ll}\text { References } & 774\end{array}$

\section{Introduction}

In characteristic zero it is a well-known and frequently used fact that the higher direct images $R^{i} f_{*} \mathrm{O}_{X}$ of a projective birational morphism $f: X \rightarrow Y$ between smooth schemes vanish for $i>0$. This statement was proved as a corollary of Hironaka's resolution of singularities by resolving the indeterminacies of $f^{-1}$ by successively blowing up smooth subvarieties of $Y$. In this article we consider the situation over an arbitrary field $k$ and prove this and related results.

In the following all schemes are assumed to be separated and of finite type over $k$, and all morphisms are assumed to be $k$-morphisms. The two main results of this paper are as follows.

This work has been supported by the SFB/TR 45 "Periods, moduli spaces and arithmetic of algebraic varieties".

MSC2000: primary 14E05; secondary 14C17.

Keywords: birational geometry, rational singularities. 
Theorem 1. Assume $k$ is perfect. Let $S$ be an arbitrary scheme and let $X$ and $Y$ be integral $S$-schemes. Assume that $X$ and $Y$ are smooth over $k$ and properly birational over $S$, that is, there exists an integral scheme $Z$ and a commutative diagram

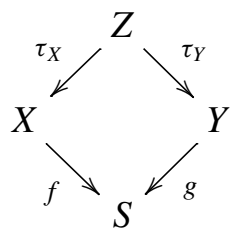

such that $\tau_{X}$ and $\tau_{Y}$ are proper and birational ( $f$ and $g$ being the fixed morphisms to $S$ ). Then for all $i$, there are isomorphisms of $\mathrm{O}_{S}$-modules

$$
R^{i} f_{*} \mathrm{O}_{X} \cong R^{i} g_{*} \mathrm{O}_{Y} \quad \text { and } \quad R^{i} f_{*} \omega_{X} \cong R^{i} g_{*} \omega_{Y} .
$$

Theorem 2. Consider a diagram

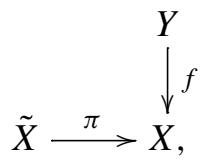

where $Y$ and $\tilde{X}$ are connected smooth schemes, $X$ is integral and normal, $f$ is surjective and finite such that $\operatorname{deg}(f) \in k^{*}$, and $\pi$ is birational and proper. Then $X$ is Cohen-Macaulay and

$$
R \pi_{*} 0_{\tilde{X}}=0_{X} \text { and } R \pi_{*} \omega_{\tilde{X}}=\omega_{X},
$$

where $\omega_{X}$ is the dualizing sheaf of $X$.

By duality, the two identities in Theorem 2 imply each other.

Both theorems are known in characteristic zero: Theorem 1 follows from Hironaka's resolution of singularities; for Theorem 2, see [Viehweg 1977] (which also uses resolution of singularities). If resolution of singularities is available in positive characteristic then it easily yields Theorem 1 .

Recall from [Kempf et al. 1973, I, Section 3, page 50] that a rational resolution of an integral normal scheme $X$ is a resolution (that is, a proper birational morphism $g: \tilde{X} \rightarrow X$ with $\tilde{X}$ smooth) that satisfies $R^{i} g_{*}\left(\mathscr{O}_{\tilde{X}}\right)=0=R^{i} g_{*}\left(\omega_{\tilde{X}}\right)$ for all $i>0$. Thus Theorem 1 implies that if an integral normal scheme over a perfect field has a rational resolution, then any resolution of $X$ is a rational resolution. For a smooth scheme $X$ we obtain $R^{i} g_{*}\left(\mathscr{O}_{\tilde{X}}\right)=0=R^{i} g_{*}\left(\omega_{\tilde{X}}\right)$ for $i>0$ and any resolution $g$ (Corollary 3.2.10). Theorem 2 asserts that $\pi: \tilde{X} \rightarrow X$ is a rational resolution; this includes the important special case where $X$ is the quotient of $Y$ by a finite group of order prime to the characteristic of $k$.

Since resolution of singularities is not yet available in positive characteristic, we develop a different approach based on algebraic correspondences. To get an idea 
of the methods involved, let us sketch the proof of Theorem 1 for $S=\operatorname{Spec} k$ and $X$ and $Y$ projective (see Section 3 for the details and a rigorous proof).

For a scheme $X$ we write $H(X)=\bigoplus_{i, j} H^{i}\left(X, \Omega_{X}^{j}\right)$ and $\mathrm{CH}(X)=\bigoplus_{i} \mathrm{CH}^{i}(X)$, with $\mathrm{CH}^{i}(X)$ the Chow group of codimension $i$ cycles on $X$. Given smooth projective schemes $X, Y, Z$, there is a composition of correspondences:

$$
\begin{gathered}
\mathrm{CH}(X \times Y) \otimes_{\mathbb{Z}} \mathrm{CH}(Y \times Z) \rightarrow \mathrm{CH}(X \times Z), \\
H(X \times Y) \otimes_{k} H(Y \times Z) \rightarrow H(X \times Z) .
\end{gathered}
$$

Moreover $a \in \mathrm{CH}(X \times Y)$ and $\in H(X \times Y)$ define maps on the cohomologies $\mathrm{CH}(X) \rightarrow \mathrm{CH}(Y)$ and $H(X) \rightarrow H(Y)$, respectively, by $c \mapsto \operatorname{pr}_{2 *}\left(\operatorname{pr}_{1}^{*}(c) \cup a\right)$, and composition of correspondences corresponds to the composition of maps. Furthermore, there is a cycle map $c l: \mathrm{CH} \rightarrow H$, which is compatible with composition.

Now, the proof proceeds as follows. By assumption there exists a closed integral subscheme $Z \subset X \times Y$ projecting birational to $X$ and $Y$. Let $Z^{\prime} \subset Y \times X$ be its transpose. By using the refined intersection product of Fulton we will see that

$$
\left[Z^{\prime}\right] \circ[Z]=\mathrm{id}_{X}+E \quad \text { in } \mathrm{CH}\left(\operatorname{pr}_{13}\left((Z \times X) \cap\left(X \times Z^{\prime}\right)\right)\right),
$$

with $E$ a cycle on $X \times X$ that projects on both sides to subsets of codimension at least one in $X$. We will show that the map defined by $c l(E) \in H(X \times X)$ acts as zero on $H^{*}\left(X, O_{X}\right) \oplus H^{*}\left(X, \omega_{X}\right)$. A similar argument applies for $[Z] \circ\left[Z^{\prime}\right]$. Thus the maps defined by $\operatorname{cl}([Z])$ and $c l\left(\left[Z^{\prime}\right]\right)$ are inverse to each other (when restricted to $\left.H^{*}\left(X, \mathscr{O}_{X}\right) \oplus H^{*}\left(X, \omega_{X}\right)\right)$. This proves Theorem 1 in the case $S=\operatorname{Spec} k$ and $X$ and $Y$ projective.

It is not hard to deduce the general statement of Theorem 1 once we know it in the case $S=\operatorname{Spec} k$. Therefore we have to generalize the argument above to the case of smooth but not necessarily proper $k$-schemes. The problem is that in general a push-forward on $\mathrm{CH}$ or $H$ does not exist. However, the variety $Z \subset X \times Y$ is proper over $X$ and $Y$, and by working with cohomology (or Chow groups) with support we can conclude as outlined above.

One of the main points in this paper is the construction of a cycle map, or natural transformation between cohomology theories with support, $\mathrm{CH} \rightarrow H$. For this, we first give a definition for (weak) cohomology theories with support. We introduce two categories $V^{*}$ and $V_{*}$. The objects in both categories are $(X, \Phi)$, where $X$ is smooth and $\Phi$ is a family of supports on $X$ (see Definition 1.1.1 for the definition of a family with supports). A morphism $f: X \rightarrow Y$ induces a morphism $(X, \Phi) \rightarrow(Y, \Psi)$ in $V_{*}$ if and only if $\left.f\right|_{\Phi}$ is proper and $f(\Phi) \subset \Psi ; f$ induces a morphism in $V^{*}$ if and only if $f^{-1}(\Psi) \subset \Phi$.

Then we consider the data $\left(F_{*}, F^{*}, T, e\right)$, where

$$
F_{*}: V_{*} \rightarrow \mathbf{G r A b} \text { and } F^{*}:\left(V^{*}\right)^{\mathrm{op}} \rightarrow \mathbf{G r A b},
$$


are functors to graded abelian groups with $F_{*}(X, \Phi)=F^{*}(X, \Phi)=: F(X, \Phi)$ as abelian groups, $T$ gives for all $(X, \Phi)$ and $(Y, \Psi)$ a morphism of abelian groups

$$
T_{(X, \Phi),(Y, \Psi)}: F(X, \Phi) \otimes_{\mathbb{Z}} F(Y, \Psi) \rightarrow F(X \times Y, \Phi \times \Psi),
$$

and $e: \mathbb{Z} \rightarrow F(\operatorname{Spec} k)$ is a morphism of abelian groups.

These data define a weak cohomology theory with support if the following conditions are satisfied.

(1) The map $T$ is functorial for $F_{*}$ and $F^{*}$.

(2) For all diagrams

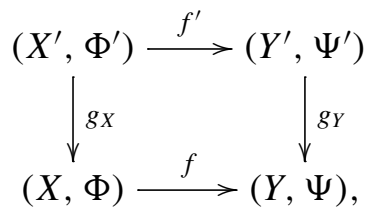

in which the underlying diagram of schemes is cartesian and transversal, with $g_{X}, g_{Y} \in V^{*}$ and $f, f^{\prime} \in V_{*}$, we have

$$
F^{*}\left(g_{Y}\right) \circ F_{*}(f)=F_{*}\left(f^{\prime}\right) \circ F^{*}\left(g_{X}\right) .
$$

(3) Some more (very natural) conditions.

The conditions allow us to obtain a calculus with correspondences. One key example of a weak cohomology theory with supports is the Chow group

$$
\mathrm{CH}(X, \Phi):=\underset{W \in \Phi}{\underset{W}{\lim }} \mathrm{CH}(W),
$$

with $\mathrm{CH}_{*}$ the (proper) push forward for cycles and $\mathrm{CH}^{*}$ the refined Gysin homomorphism. Another example is the Hodge cohomology

$$
H(X, \Phi):=\bigoplus_{i, j} H_{\Phi}^{i}\left(X, \Omega_{X}^{j}\right) .
$$

Here, the definition of $H^{*}$ is straightforward, but for $H_{*}$ we use Grothendieck duality for singular schemes since smooth compactifications are not available in characteristic $p$. That the Hodge cohomology defines a (weak) cohomology theory with supports is a nontrivial fact, the proof of which occupies Section 2.

In Theorem 1.2.3 we give necessary and sufficient conditions for a (weak) cohomology theory with supports $F$ to be target of a morphism from $\mathrm{CH}$. Unfortunately, we can do this only with an additional semipurity assumption on $F$ (see Definition 1.2.1). As an application we prove the existence of a cycle map $\mathrm{CH} \rightarrow H$. We hope that Theorem 1.2.3 will turn out to be useful for proving similar results for the Witt vector cohomology.

Let us give a short overview of the content of each section. 
In Section 1 we define weak cohomology theories with supports and prove basic properties. We show that $\mathrm{CH}$ is an example and prove Theorem 1.2.3. Moreover, we explain the calculus of correspondences attached to a cohomology theory.

In Section 2 we show that the Hodge cohomology is another example for a cohomology theory with supports. The hard part is the definition of push-forward maps. We use Grothendieck's duality theory for singular schemes as developed in [Hartshorne 1966; Conrad 2000], and make extensive use of the results given in these references. There are also other approaches to duality theory that are more elegant (see for example [Lipman and Hashimoto 2009]). But since we use at several places the explicit description of duality theory as developed by Grothendieck and since it is not clear to the authors how this classical approach compares to the one, for example, in [Lipman and Hashimoto 2009], we will solely stick to the references [Hartshorne 1966; Conrad 2000].

In Section 3 we show the existence of a cycle map $\mathrm{CH} \rightarrow H$. We also prove a vanishing statement Proposition 3.2.2, enabling us to prove Theorem 1 and 2.

In Section 4 we generalize Theorem 1 to the case where $X$ and $Y$ are tame quotients (see Theorem 4.3.1). This theorem also implies Theorem 2.

We finish with some open questions.

In the appendix we describe the trace morphism for closed embeddings between smooth schemes and for finite and surjective morphisms between smooth schemes; this is well known but needed in Section 2.

\section{Chow groups with support}

1.1. Cohomology theories with support. Let $k$ be a field. We assume all schemes are of finite type and separated over $k$. We begin by recalling basic definitions and notation concerning families of supports.

Definition 1.1.1. A family of supports $\Phi$ on $X$ is a nonempty set of closed subsets of $X$ such that the following holds:

(i) The union of two elements in $\Phi$ is contained in $\Phi$.

(ii) Every closed subset of an element in $\Phi$ is contained in $\Phi$.

Let $A$ be any set of closed subsets of $X$. The smallest family of supports $\Phi_{A}$ that contains $A$ is given by

$$
\Phi_{A}:=\left\{\bigcup_{i=1}^{n} Z_{i}^{\prime} ; Z_{i}^{\prime} \underset{\text { closed }}{\subset} Z_{i} \in A\right\} .
$$

For a closed subset $Z \subset X$ we write $\Phi_{Z}$ for $\Phi_{\{Z\}}$.

Notation 1.1.3. Let $f: X \rightarrow Y$ be a morphism of schemes and let $\Phi$ and $\Psi$ be families of supports of $X$ and $Y$, respectively. 
(1) We denote by $f^{-1}(\Psi)$ the smallest family of supports on $X$ that contains $\left\{f^{-1}(Z): Z \in \Psi\right\}$.

(2) We say that $\left.f\right|_{\Phi}$ is proper if $\left.f\right|_{Z}$ is proper for every $Z \in \Phi$. If $\left.f\right|_{\Phi}$ is proper, then $f(\Phi)$ is a family of supports on $Y$.

(3) If $\Phi_{1}$ and $\Phi_{2}$ are two families of supports, then $\Phi_{1} \cap \Phi_{2}$ is a family of supports.

(4) If $\Phi$ and $\Psi$ are families of supports of $X$ and $Y$, respectively, then we denote by $\Phi \times \Psi$ the smallest family of supports on $X \times_{k} Y$ that contains

$$
\left\{Z_{1} \times Z_{2} ; Z_{1} \in \Phi, Z_{2} \in \Psi\right\}
$$

When working with cohomology theories with support, it is convenient to define the following two categories $V_{*}$ and $V^{*}$, where for the morphisms in $V_{*}$ a "pushforward" map can be expected and for the morphisms in $V^{*}$ a "pull-back" map can be expected.

Definition 1.1.4. We denote by $V_{*}$ the category with objects $(X, \Phi)$, where $X$ is a smooth scheme and $\Phi$ is a family of supports of $X$, and morphisms

$$
\operatorname{Hom}_{V_{*}}((X, \Phi),(Y, \Psi))=\left\{f \in \operatorname{Hom}_{k}(X, Y) ;\left.f\right|_{\Phi} \text { is proper and } f(\Phi) \subset \Psi\right\} \text {. }
$$

We denote by $V^{*}$ the category with objects $(X, \Phi)$, where $X$ is a smooth scheme and $\Phi$ is a family of supports of $X\left(\mathrm{ob}\left(V_{*}\right)=\mathrm{ob}\left(V^{*}\right)\right)$, and morphisms

$$
\operatorname{Hom}_{V^{*}}((X, \Phi),(Y, \Psi))=\left\{f \in \operatorname{Hom}_{k}(X, Y) ; f^{-1}(\Psi) \subset \Phi\right\} .
$$

The composition and the identity comes in both cases from the category of schemes (over $k$ ).

1.1.5. Let $X$ be a smooth scheme. For a closed subscheme $W \subset X$ we write $(X, W):=\left(X, \Phi_{W}\right)$ in $V^{*}$ and $V_{*}$, respectively. We simply write $X$ for $(X, X)$.

We respectively have forgetful functors $V_{*} \rightarrow \mathbf{S c h}_{k}$ and $V^{*} \rightarrow \mathbf{S c h}_{k}$ to the category of schemes, and we often denote the morphism of schemes induced by a morphism in $V_{*}$ and $V^{*}$, respectively, by the same letter.

For a morphism $f$ in $V_{*}$ we will say that $f$ is an immersion, flat, ..., if the corresponding morphism of schemes has this property, and similarly for morphisms in $V^{*}$. We say that a diagram

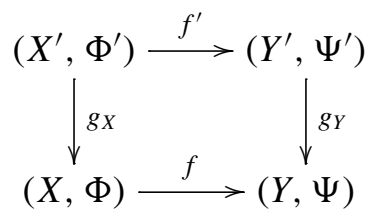

is cartesian if the diagram of the corresponding schemes is cartesian. 
1.1.7. Coproducts and "products". For both categories $V^{*}$ and $V_{*}$, finite coproducts exist:

$$
(X, \Phi) \bigsqcup(Y, \Psi)=(X \bigsqcup Y, \Phi \cup \Psi) .
$$

For $(X, \Phi)$, let $X=\bigsqcup_{i} X_{i}$ be the decomposition into connected components; then

$$
(X, \Phi)=\coprod_{i}\left(X_{i}, \Phi \cap \Phi_{X_{i}}\right) .
$$

In general products don't exist, and we define

$$
(X, \Phi) \otimes(Y, \Psi):=(X \times Y, \Phi \times \Psi),
$$

which together with the unit object $\mathbf{1}=\operatorname{Spec}(k)$ and the obvious isomorphism $(X, \Phi) \otimes(Y, \Psi) \rightarrow(Y, \Psi) \otimes(X, \Phi)$ makes $V_{*}$ and $V^{*}$ into a symmetric monoidal category; see [Mac Lane 1998, VII.1].

1.1.8. Consider the following data $\left(F_{*}, F^{*}, T, e\right)$ :

(1) Two functors $F_{*}: V_{*} \rightarrow \mathbf{G r A b}$ and $F^{*}:\left(V^{*}\right)^{\text {op }} \rightarrow \mathbf{G r A b}$ to the symmetric monoidal category of graded abelian groups, such that $F_{*}(X)=F^{*}(X)$ as ungraded groups for every object $X \in \mathrm{ob}\left(V_{*}\right)=\mathrm{ob}\left(V^{*}\right)$. We will simply write $F(X):=F_{*}(X)=F^{*}(X)$. We use lower indexes for the grading on $F_{*}(X)$, that is, $F_{*}(X)=\oplus_{i} F_{i}(X)$, and upper indexes for $F^{*}(X)$.

(2) For every two objects $X, Y \in \mathrm{ob}\left(V_{*}\right)=\mathrm{ob}\left(V^{*}\right)$, a morphism of graded abelian groups (for both gradings):

$$
T_{X, Y}: F(X) \otimes_{\mathbb{Z}} F(Y) \rightarrow F(X \otimes Y) .
$$

(3) A morphism of abelian groups $e: \mathbb{Z} \rightarrow F(\operatorname{Spec}(k))$. For all smooth schemes $\pi: X \rightarrow \operatorname{Spec}(k)$ we denote by $1_{X}$ the image of $1 \in \mathbb{Z}$ via the map

$$
\mathbb{Z} \stackrel{e}{\rightarrow} F^{*}(\operatorname{Spec}(k)) \stackrel{F^{*}(\pi)}{\longrightarrow} F^{*}(X) .
$$

1.1.9. The data $\left(F_{*}, F^{*}, T, e\right)$ is called a weak cohomology theory with supports if the following conditions are satisfied:

(1) The functor $F_{*}$ preserves coproducts and $F^{*}$ maps coproducts to products, and for $\left(X, \Phi_{1}\right),\left(X, \Phi_{2}\right) \in \mathrm{ob}\left(V_{*}\right)$ with $\Phi_{1} \cap \Phi_{2}=\{\varnothing\}$, the map

$$
F^{*}\left(J_{1}\right)+F^{*}\left(J_{2}\right): F^{*}\left(X, \Phi_{1}\right) \oplus F^{*}\left(X, \Phi_{2}\right) \rightarrow F^{*}\left(X, \Phi_{1} \cup \Phi_{2}\right),
$$

with $J_{1}:\left(X, \Phi_{1} \cup \Phi_{2}\right) \rightarrow\left(X, \Phi_{1}\right)$ and $J_{2}:\left(X, \Phi_{1} \cup \Phi_{2}\right) \rightarrow\left(X, \Phi_{2}\right)$ in $V^{*}$, is an isomorphism.

(2) The data $\left(F_{*}, T, e\right)$ and $\left(F^{*}, T, e\right)$ respectively define a (right-lax) symmetric monoidal functor (see below). 
(3) Grading: For $(X, \Phi)$ such that $X$ is connected, we have

$$
F_{i}(X, \Phi)=F^{2 \operatorname{dim} X-i}(X, \Phi) \text { for all } i .
$$

(4) For all cartesian diagrams (1.1.6) with $g_{X}, g_{Y} \in V^{*}$ and $f, f^{\prime} \in V_{*}$ such that either $g_{Y}$ is smooth or $g_{Y}$ is a closed immersion and $f$ is transversal to $g_{Y}$, we have

$$
F^{*}\left(g_{Y}\right) \circ F_{*}(f)=F_{*}\left(f^{\prime}\right) \circ F^{*}\left(g_{X}\right) .
$$

Recall that $f$ is transversal to $g_{Y}$ if $\left(f^{\prime}\right)^{*} N_{Y^{\prime} / Y}=N_{X^{\prime} / X}$, where $N$ denotes the normal bundle. The case $X^{\prime}=\varnothing$ is also admissible; in this case the equality 1.1.9(4) reads $F^{*}\left(g_{Y}\right) \circ F_{*}(f)=0$. The condition 1.1.9(4) implies the projection formula (see Proposition 1.1.16) and will be needed for a calculus with correspondences.

Recall that $\left(F_{*}, T, e\right)$ is called a right-lax symmetric monoidal functor if

- $T$ is associative, that is, for $X, Y, Z \in \mathrm{ob}\left(V_{*}\right)$, the diagram

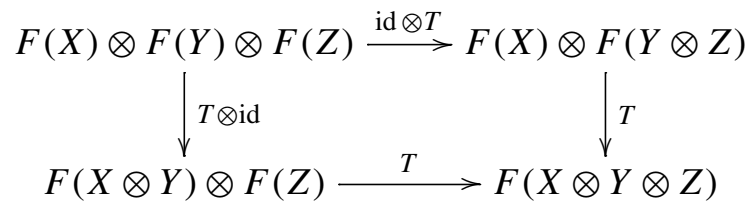

is commutative;

- $T$ is commutative, that is, for $X, Y \in \mathrm{ob}\left(V_{*}\right)$, the diagram

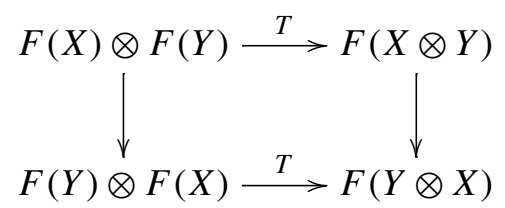

is commutative. Here, for two graded abelian groups $A$ and $B$, the morphism $A \otimes B \rightarrow B \otimes A$ maps $a \otimes b \mapsto(-1)^{\operatorname{deg}(a) \operatorname{deg}(b)} b \otimes a ;$

- the map $e: \mathbb{Z} \rightarrow F(\operatorname{Spec}(k))$ renders commutative the diagrams

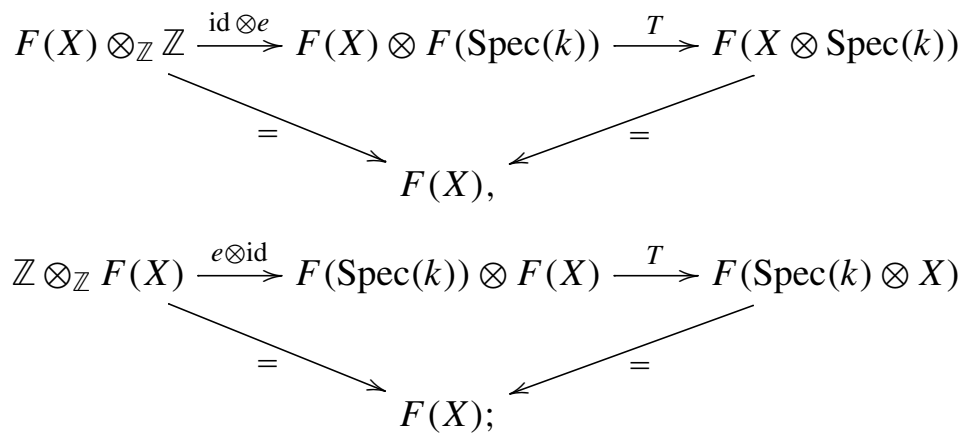


- $T$ is a natural transformation

$$
T:\left(V_{*} \times V_{*} \stackrel{F_{*} \times F_{*}}{\rightarrow} \mathbf{G r A b} \times \mathbf{G r A b} \stackrel{\otimes}{\rightarrow} \mathbf{G r A b}\right) \rightarrow\left(V_{*} \times V_{*} \stackrel{\otimes}{\rightarrow} V_{*} \stackrel{F_{*}}{\rightarrow} \mathbf{G r A b}\right) .
$$

Example 1.1.10. The Chow group

$$
(X, \Phi) \mapsto \lim _{W \in \Phi} \mathrm{CH}_{*}(W)
$$

satisfies these conditions (see Proposition 1.1.34). The push-forward $V_{*} \rightarrow \mathbf{G r A b}$ is defined in the usual way. To define the pull-back $\left(V^{*}\right)^{\mathrm{op}} \rightarrow \mathbf{G r A b}$, we use Fulton's refined Gysin homomorphism. However, in order to get a symmetric functor we have to put the Chow group $\mathrm{CH}_{d}(W)$ in degree $=2 d$.

It will be shown in Section 2 that the Hodge cohomology with support

$$
(X, \Phi) \mapsto \bigoplus_{i, j} H_{\Phi}^{i}\left(X, \Omega_{X}^{j}\right)
$$

is another example. The push-forward is an application of Grothendieck's duality theory.

An example not further considered in this paper is the étale cohomology with support

$$
(X, \Phi) \mapsto H_{\Phi}^{*}\left(X \times \bar{k}, \mathbb{Q}_{\ell}\right) .
$$

Definition 1.1.11. Let $\left(F_{*}, F^{*}, T, e\right)$ and $\left(G_{*}, G^{*}, U, \epsilon\right)$ be as in 1.1 .8 and satisfy the conditions 1.1.9. By a morphism

$$
\left(F_{*}, F^{*}, T, e\right) \rightarrow\left(G_{*}, G^{*}, U, \epsilon\right),
$$

we mean a morphism of graded abelian groups (for both gradings)

$$
\phi: F(X) \rightarrow G(X) \text { for every } X \in \mathrm{ob}\left(V_{*}\right)=\mathrm{ob}\left(V^{*}\right),
$$

such that $\phi$ induces a natural transformation of (right-lax) symmetric monoidal functors

$$
\phi:\left(F_{*}, T, e\right) \rightarrow\left(G_{*}, U, \epsilon\right) \quad \text { and } \quad \phi:\left(F^{*}, T, e\right) \rightarrow\left(G^{*}, U, \epsilon\right) \text {, }
$$

that is, $\phi$ induces natural transformations $F_{*} \rightarrow G_{*}, F^{*} \rightarrow G^{*}$, and

$$
\phi \circ T=U \circ(\phi \otimes \phi), \quad \phi \circ e=\epsilon .
$$

We denote by $\mathbf{T}$ the category of weak cohomology theories with supports, that is, it is the category consisting of objects $\left(F_{*}, F^{*}, T, e\right)$ as in 1.1 .8 , and satisfying the properties 1.1.9, together with morphisms (1.1.12). 
1.1.14. Cup product. Let $\left(F_{*}, F^{*}, T, e\right) \in \mathbf{T}$. For all $(X, \Phi) \in \mathrm{ob}\left(V^{*}\right)$ we obtain a cup product

$\cup: F\left(X, \Phi_{1}\right) \otimes F\left(X, \Phi_{2}\right) \stackrel{T}{\rightarrow} F\left(X \times X, \Phi_{1} \times \Phi_{2}\right) \stackrel{F^{*}\left(\Delta_{X}\right)}{\longrightarrow} F\left(X, \Phi_{1} \cap \Phi_{2}\right)$, where $\Delta_{X}:\left(X, \Phi_{1} \cap \Phi_{2}\right) \rightarrow\left(X \times X, \Phi_{1} \times \Phi_{2}\right)$ is induced by the diagonal immersion. The cup product is associative and graded commutative.

By functoriality we obtain

$$
F^{*}\left(f_{1}\right)(a) \cup F^{*}\left(f_{2}\right)(b)=F^{*}\left(f_{3}\right)(a \cup b)
$$

for all morphisms $f_{1}:\left(X^{\prime}, \Phi_{1}^{\prime}\right) \rightarrow\left(X, \Phi_{1}\right), f_{2}:\left(X^{\prime}, \Phi_{2}^{\prime}\right) \rightarrow\left(X, \Phi_{2}\right)$ in $V^{*}$ with $f_{1}=f_{2}:=f$ as morphisms of schemes; $f_{3}:\left(X^{\prime}, \Phi_{1}^{\prime} \cap \Phi_{2}^{\prime}\right) \rightarrow\left(X, \Phi_{1} \cap \Phi_{2}\right)$ in $V^{*}$ is induced by $f$.

Proposition 1.1.16 (projection formula). Let $\left(F_{*}, F^{*}, T, e\right) \in \mathbf{T}$ and let $f: X \rightarrow Y$ be a morphism between smooth schemes, inducing morphisms

$$
\begin{aligned}
& f_{1}:\left(X, \Phi_{1}\right) \rightarrow\left(Y, \Phi_{2}\right) \text { in } V_{*}, \\
& f_{2}:\left(X, f^{-1}(\Psi)\right) \rightarrow(Y, \Psi) \quad \text { in } V^{*} .
\end{aligned}
$$

Then $f$ also induces a morphism

$$
f_{3}:\left(X, \Phi_{1} \cap f^{-1}(\Psi)\right) \rightarrow\left(Y, \Phi_{2} \cap \Psi\right) \quad \text { in } V_{*}
$$

and for all $a \in F\left(X, \Phi_{1}\right)$ and $b \in F(Y, \Psi)$ we have in $F\left(Y, \Phi_{2} \cap \Psi\right)$

$$
\begin{aligned}
& F_{*}\left(f_{3}\right)\left(a \cup F^{*}\left(f_{2}\right)(b)\right)=F_{*}\left(f_{1}\right)(a) \cup b, \\
& F_{*}\left(f_{3}\right)\left(F^{*}\left(f_{2}\right)(b) \cup a\right)=b \cup F_{*}\left(f_{1}\right)(a) .
\end{aligned}
$$

Proof. We prove the first equality of the statement; the second is proved in the same way. The diagram

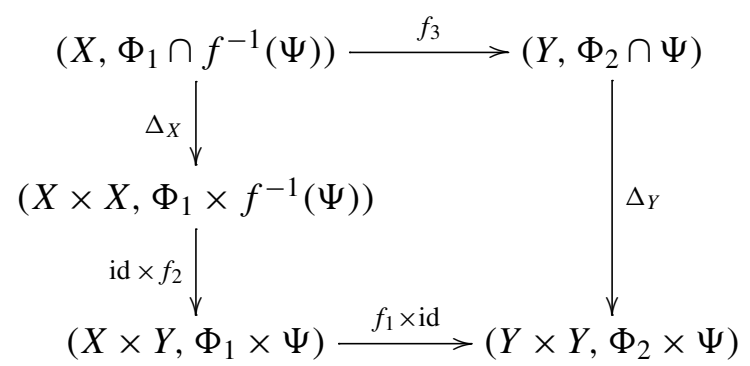

is cartesian and $f \times$ id is transversal to $\Delta_{Y}$. Thus by 1.1.9(4) we get

$$
\begin{aligned}
F_{*}\left(f_{3}\right)\left(a \cup F^{*}\left(f_{2}\right)(b)\right) & =F_{*}\left(f_{3}\right) F^{*}\left(\Delta_{X}\right) F^{*}\left(\mathrm{id} \times f_{2}\right)(T(a \otimes b)) \\
& =F^{*}\left(\Delta_{Y}\right) F_{*}\left(f_{1} \times \mathrm{id}\right)(T(a \otimes b))=F_{*}\left(f_{1}\right)(a) \cup b .
\end{aligned}
$$

The proof of the following lemma is straightforward. 
Lemma 1.1.17. (1) For all $(X, \Phi)$ and $a \in F(X, \Phi)$ the equality

$$
1_{X} \cup a=a=a \cup 1_{X}
$$

holds. In particular $F^{*}(X)$ is a (graded) ring.

(2) For smooth schemes $X$ and $Y$, we have

$$
T\left(1_{X} \otimes 1_{Y}\right)=1_{X \times Y} .
$$

1.1.18. Definition of Chow groups with support. In the following we define a first example of an object $\left(\mathrm{CH}_{*}, \mathrm{CH}^{*}, \times, e\right) \in \mathbf{T}$.

Definition 1.1.19 (Chow groups with support). Let $\Phi$ be a family of supports on $X$. We define

$Z_{\Phi}(X):=$ abelian group freely generated by irreducible closed subsets $Z \in \Phi$;

$\operatorname{Rat}_{\Phi}(X):=$ subgroup of $Z_{\Phi}(X)$ generated by $\operatorname{div}(f)$, where $f \in k(W)^{*}$ is a nonzero rational function and $W \in \Phi$ is irreducible;

$\mathrm{CH}(X, \Phi):=Z_{\Phi}(X) / \operatorname{Rat} \Phi(X)$.

For $(X, \Phi)$ and $(Y, \Psi)$ we obtain

$$
\mathrm{CH}((X \bigsqcup Y, \Phi \cup \Psi))=\mathrm{CH}(X, \Phi) \oplus \mathrm{CH}(Y, \Psi) .
$$

1.1.21. Grading. The groups $Z_{\Phi}(X)$ and $\operatorname{Rat} \Phi(X)$ can be graded by dimension:

$$
\mathrm{CH}_{*}(X, \Phi)=\bigoplus_{d \geq 0} \mathrm{CH}_{d}(X, \Phi)[2 d],
$$

where the bracket $[2 d]$ means that $\mathrm{CH}_{d}(X, \Phi)$ is considered to be in degree $2 d$.

There is also a grading by codimension. Let $X=\bigsqcup_{i} X_{i}$ be the decomposition into connected components. Then $\mathrm{CH}^{*}(X, \Phi)=\bigoplus_{i} \mathrm{CH}^{*}\left(X_{i}, \Phi \cap \Phi_{X_{i}}\right)$ and

$$
\mathrm{CH}^{*}\left(X_{i}, \Phi \cap \Phi_{X_{i}}\right)=\bigoplus_{d \geq 0} \mathrm{CH}^{d}\left(X_{i}, \Phi \cap \Phi_{X_{i}}\right)[2 d],
$$

where $\mathrm{CH}^{d}\left(X_{i}, \Phi\right)$ is generated by cycles [ $\left.Z\right]$ with $Z \in \Phi \cap \Phi_{X}, \quad Z$ irreducible, and $\operatorname{codim}_{X_{i}}(Z)=d$.

1.1.22. Examples. If $W \subset X$ is a closed subset, then we get

$$
\mathrm{CH}\left(X, \Phi_{W}\right)=\mathrm{CH}(X, W)=\mathrm{CH}(W),
$$

the usual Chow group of $W$.

If $X$ is proper, $U$ is affine, and $\Phi:=\left\{Z^{\prime} ; Z^{\prime} \subset U\right\}$, then

$\mathrm{CH}(X, \Phi)=Z_{\Phi}(X)=$ freely generated by closed points of $U$. 
1.1.23. Push forward for Chow groups. Let $\Phi$ be a family of supports on $X$ as in Definition 1.1.1. If $W \subset X$ is a closed subscheme with $W \in \Phi$, then $Z(W)=$ $Z_{\Phi_{W}}(X) \subset Z_{\Phi}(X)$, Rat $(W)=\operatorname{Rat} \Phi_{W}(X) \subset \operatorname{Rat} \Phi(X)$ (with $\Phi_{W}$ as defined in (1.1.2)), and we obtain a map

$$
\mathrm{CH}(W)=\mathrm{CH}(X, W) \rightarrow \mathrm{CH}(X, \Phi) .
$$

Obviously, $\mathrm{CH}(X, \Phi)$ is the largest quotient of $Z_{\Phi}(X)$ such that there are pushforward maps (1.1.24) for every $W \in \Phi$.

1.1.25. In general, let $f:(X, \Phi) \rightarrow(Y, \Psi)$ be a morphism in $V_{*}$. There is a push forward of cycles

$$
f_{*}: Z_{\Phi}(X) \rightarrow Z_{\Psi}(Y) \quad \text { and } \quad f_{*}([Z])=\operatorname{deg}(Z / f(Z)) \cdot[f(Z)],
$$

for $Z \in \Phi$ irreducible $(\operatorname{deg}(Z / f(Z))=0$ if $\operatorname{dim}(f(Z))<\operatorname{dim}(Z))$. Push forward is functorial [Fulton 1998, Section 1.4].

Lemma 1.1.26. With the assumption of 1.1.25, we get $f_{*}(\operatorname{Rat} \Phi(X)) \subset \operatorname{Rat} \Psi(Y)$.

Proof. Indeed, Rat $\Phi(X)$ is generated by the images of Rat $(W)$ where $W \in \Phi$. The restriction $\left.f\right|_{W}$ is proper and [Fulton 1998, Proposition 1.4] yields

$$
f_{*}(\operatorname{Rat}(W)) \subset \operatorname{Rat}(f(W)) .
$$

Thus we get an induced map

$$
f_{*}: \mathrm{CH}(X, \Phi) \rightarrow \mathrm{CH}(Y, \Psi)
$$

and a functor

$$
\mathrm{CH}_{*}: V_{*} \rightarrow \mathbf{G r A b}, \quad \mathrm{CH}_{*}(X, \Phi):=\mathrm{CH}(X, \Phi), \quad \mathrm{CH}_{*}(f):=f_{*} .
$$

Proposition 1.1.29. Let $\Phi$ be a family of supports of $X$. The map

$$
\lim _{W \in \Phi} \mathrm{CH}(X, W) \rightarrow \mathrm{CH}(X, \Phi)
$$

is an isomorphism.

Proof. This is straightforward.

1.1.30. Pull-back for Chow groups. To define a functor

$$
\mathrm{CH}^{*}:\left(V^{*}\right)^{\mathrm{op}} \rightarrow \mathbf{G r A b}
$$

we recall Fulton's work on refined Gysin morphisms [Fulton 1998, Section 6.6].

Let $f: X \rightarrow Y$ be a morphism between smooth schemes and let $V \subset Y$ be a closed subscheme. There is a morphism

$$
f^{!}: \mathrm{CH}(V) \rightarrow \mathrm{CH}\left(f^{-1}(V)\right)
$$


of abelian groups (where $f^{-1}(V)=X \times_{Y} V$ ) with the following properties:

(1) For a closed subscheme $V^{\prime} \subset Y$ with $V \subset V^{\prime}$ (denote the immersion by $\imath$ and the immersion $f^{-1}(V) \subset f^{-1}\left(V^{\prime}\right)$ by $\left.\mathrm{J}\right)$, we have the equality

$$
f^{!} l_{*}=J_{*} f^{!}
$$

(as maps $\mathrm{CH}(V) \rightarrow \mathrm{CH}\left(f^{-1}\left(V^{\prime}\right)\right)$ ).

(2) If $g: Y \rightarrow Z$ is another morphism between smooth schemes and $S \subset Z$ a closed subscheme, then

$$
f^{!} \circ g^{!}=(g \circ f)^{!}
$$

as maps $\mathrm{CH}(S) \rightarrow \mathrm{CH}\left((g \circ f)^{-1}(S)\right)$.

(3) If $f: X \rightarrow Y$ is flat, then $f^{!}=f^{*}$ where $f^{*}$ is the usual pull-back map for flat morphisms.

(4) Let

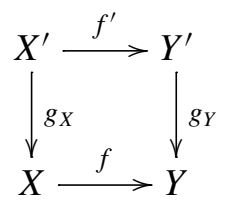

be a cartesian diagram of smooth schemes and $W \subset X$ a closed subscheme such that $\left.f\right|_{W}$ is proper. Assume that either $g_{Y}$ is flat or $g_{Y}$ is a closed immersion and $f$ is transversal to $g_{Y}$. Then

$$
g_{Y}^{!} f_{*}=f_{*}^{\prime} g_{X}^{!}
$$

as maps $\mathrm{CH}(W) \rightarrow \mathrm{CH}\left(f^{\prime}\left(g_{X}^{-1} W\right)\right)=\mathrm{CH}\left(g_{Y}^{-1} f(W)\right)$. This statement is proved in [Fulton 1998, Proposition 1.7] for flat morphisms and in [Fulton 1998, Theorem 6.2(a),(c)] for the case of a closed immersion.

Remark 1.1.31. Note that $\mathrm{CH}(W)=\mathrm{CH}\left(W_{\text {red }}\right)$ for every scheme $W$.

1.1.32. Definition of the pull-back map. Let $f: X \rightarrow Y$ be a morphism between smooth schemes and let $V \subset Y$ be a closed subscheme; thus $f:\left(X, f^{-1}(V)\right) \rightarrow$ $(Y, V)$ is a morphism in $V^{*}$. We define

$$
\mathrm{CH}^{*}(f):=f^{!}: \mathrm{CH}(Y, V)=\mathrm{CH}(V) \rightarrow \mathrm{CH}\left(f^{-1}(V)\right)=\mathrm{CH}\left(X, f^{-1}(V)\right) .
$$

For the general case, let $f:(X, \Phi) \rightarrow(Y, \Psi)$ be any morphism in $V^{*}$. For every $V \in \Psi$ the map $f$ induces $\left(X, f^{-1}(V)\right) \rightarrow(Y, V)$ in $V^{*}$. Because of 1.1.30(1) and Proposition 1.1.29, we obtain

$$
\mathrm{CH}^{*}(f): \mathrm{CH}(Y, \Psi)=\varliminf_{V \in \Psi} \mathrm{CH}(Y, V) \rightarrow \underline{\lim }_{W \in \Phi} \mathrm{CH}(X, W)=\mathrm{CH}(X, \Phi) .
$$


The assignment

$\mathrm{CH}^{*}:\left(V^{*}\right)^{\mathrm{op}} \rightarrow \mathbf{G r A b}, \quad \mathrm{CH}^{*}(X, \Phi)=\mathrm{CH}(X, \Phi), \quad f \mapsto \mathrm{CH}^{*}(f)$,

defines a functor by 1.1.30(1) and (2).

Proposition 1.1.34. With the exterior product $\times$ (see [Fulton 1998, Section 1.10]) and the obvious unit $1: \mathbb{Z} \rightarrow \mathrm{CH}(\operatorname{Spec}(k))$, we obtain an object

$$
\left(\mathrm{CH}_{*}, \mathrm{CH}^{*}, \times, e\right) \in \mathbf{T} \text {. }
$$

Proof. The formula 1.1.9(4) follows from 1.1.30(1) and (4).

1.2. Chow groups with support as initial object. Given $\left(\mathrm{CH}_{*}, \mathrm{CH}^{*}, \times, 1\right)$ we are interested in objects $\left(F_{*}, F^{*}, T, e\right) \in \mathbf{T}$ that admit a morphism $\left(\mathrm{CH}_{*}, \mathrm{CH}^{*}, \times, 1\right) \rightarrow$ $\left(F_{*}, F^{*}, T, e\right)$. Such morphisms should be viewed as a kind of cycle map, which is compatible with push-forward and pull-back. Unfortunately, we can only give a satisfactory answer under an additional hypothesis on $\left(F_{*}, F^{*}, T, e\right)$, which we call semipurity.

Definition 1.2.1 (semipurity). We say that $\left(F_{*}, F^{*}, T, e\right)$ satisfies the semipurity condition if the following holds:

- For all smooth schemes $X$ and irreducible closed subsets $W \subset X$, the groups $F_{i}(X, W)$ vanish if $i>2 \operatorname{dim} W$.

- For all smooth schemes $X$, closed subsets $W \subset X$, and open sets $U \subset X$ such that $U$ contains the generic point of every irreducible component of $W$, we require the map

$$
F^{*}(\jmath): F_{2 \operatorname{dim} W}(X, W) \rightarrow F_{2 \operatorname{dim} W}(U, W \cap U),
$$

induced by $\mathrm{J}:(U, W \cap U) \rightarrow(X, W)$ in $V^{*}$, to be injective.

Remark 1.2.2. For $\left(\mathrm{CH}_{*}, \mathrm{CH}^{*}, \times, 1\right)$ the condition is satisfied since

$\mathrm{CH}_{2 \operatorname{dim} W}(X, W)=\mathbb{Z} \cdot[W]$ and $\mathrm{CH}_{i}(X, W)=0$ for $i>2 \operatorname{dim} W$.

Let $c$ be the codimension of $W$ in $X$, so that $F_{2 \operatorname{dim} W}=F^{2 c}$. Whenever there are exact sequences

$$
F^{2 c}(X, W \backslash U) \rightarrow F^{2 c}(X, W) \rightarrow F^{2 c}(U, U \cap W),
$$

the conditions in 1.2.1 follow from $F^{i}(X, W)=0$ for $i<2 c$ (and all pairs $(X, W)$ ); this is known as semipurity in the literature.

Theorem 1.2.3. Suppose $k$ is a perfect field and assume $\left(F_{*}, F^{*}, T, e\right) \in \mathbf{T}$ satisfies the semipurity condition 1.2.1. Then $\operatorname{Hom}_{\mathbf{T}}\left(\left(\mathrm{CH}_{*}, \mathrm{CH}^{*}, \times, 1\right),\left(F_{*}, F^{*}, T, e\right)\right)$ is either empty or contains only one element; it is nonempty if and only if the following conditions hold: 
(1) If $f: X \rightarrow Y$ is a finite morphism between smooth connected schemes of equal dimension, then

$$
F_{*}(f)\left(1_{X}\right)=\operatorname{deg}(f) \cdot 1_{Y} .
$$

(2) For the 0-point $\iota_{0}: \operatorname{Spec}(k) \rightarrow \mathbb{P}^{1}$ and the $\infty$-point $\iota_{\infty}: \operatorname{Spec}(k) \rightarrow \mathbb{P}^{1}$ the following equality holds:

$$
F_{*}\left(l_{0}\right) \circ e=F_{*}\left(l_{\infty}\right) \circ e .
$$

(3) For a closed immersion $\imath: X \rightarrow Y$ between smooth schemes and an effective smooth divisor $D \subset Y$ such that

- D meets $X$ properly and thus $D \cap X:=D \times_{Y} X$ is a divisor on $X$,

- $D^{\prime}:=(D \cap X)_{\text {red }}$ is smooth and connected and thus $D \cap X=n \cdot D^{\prime}$ as divisors (for some $n \in \mathbb{Z}, n \geq 1$ ),

we denote by $\iota_{X}: X \rightarrow(Y, X)$ and $\iota_{D^{\prime}}: D^{\prime} \rightarrow\left(D, D^{\prime}\right)$ the morphisms in $V_{*}$ induced by $\iota$, and we define $g:\left(D, D^{\prime}\right) \rightarrow(Y, X)$ in $V^{*}$ by the inclusion $D \subset Y$. Then the following equality is required to hold:

$$
F^{*}(g)\left(F_{*}\left(l_{X}\right)\left(1_{X}\right)\right)=n \cdot F_{*}\left(l_{D^{\prime}}\right)\left(1_{D^{\prime}}\right) .
$$

(4) If $W \subset X$ is an irreducible closed subset, then there is an element $c l_{(X, W)} \in$ $F_{2 \operatorname{dim} W}(X, W)$ with

$$
F^{*}(J)\left(c l_{(X, W)}\right)=F_{*}(\iota)\left(1_{U \cap W}\right)
$$

for all open sets $U \subset X$ such that $U \cap W \neq \varnothing$ is smooth, and where $\mathrm{J}$ : $(U, W \cap U) \rightarrow(X, W)$ in $V^{*}$ and $\iota: W \cap U \rightarrow(U, W \cap U)$ in $V_{*}$.

We will give the proof after the proof of the following proposition.

Proposition 1.2.4. Let $k$ be a perfect field and let $F:=\left(F_{*}, F^{*}, T, e\right) \in \mathbf{T}$ satisfy the semipurity condition 1.2.1. We also assume that the conditions (1)-(4) of Theorem 1.2.3 hold for $F$. Then there is a unique natural transformation of (right-lax) symmetric monoidal functors

$$
\phi:\left(\mathrm{CH}_{*}, \times, 1\right) \rightarrow\left(F_{*}, T, e\right)
$$

such that $\phi\left(1_{X}\right)=1_{X}$ for every smooth scheme $X$.

Proof. Uniqueness: In view of the semipurity condition 1.2.1,

$$
\phi([W])=c l_{(X, W)}
$$

is the only choice for an irreducible closed subset $W$ of $X,[W] \in \mathrm{CH}_{*}(X, W)$. For a general family of supports $\Phi$ of $X$, the group $\mathrm{CH}_{*}(X, \Phi)$ is generated by the images of $[W]$ via $\mathrm{CH}_{*}(X, W) \rightarrow \mathrm{CH}_{*}(X, \Phi)$, where $W$ runs through all irreducible closed subsets $W \in \Phi$. 
Existence: For every smooth scheme $X$ and a family of supports $\Phi$ of $X$, we define a homomorphism of abelian groups

$$
\phi_{(X, \Phi)}^{\prime}: Z_{\Phi}(X) \rightarrow F(X, \Phi)
$$

by $\phi_{(X, \Phi)}^{\prime}([W])=F_{*}\left(l_{W}\right)\left(c l_{(X, W)}\right)$ for every irreducible closed subset $W \in \Phi$ and $\iota_{W}:(X, W) \rightarrow(X, \Phi)$ in $V_{*}$ induced by $\operatorname{id}_{X}$.

1st step: For every morphism $f:(X, \Phi) \rightarrow(Y, \Psi)$ in $V_{*}$ the push-forward $f_{*}$ : $Z_{\Phi}(X) \rightarrow Z_{\Psi}(Y)$ is well defined by 1.1.25. We claim that

$$
\phi_{(Y, \Psi)}^{\prime} \circ f_{*}=F_{*}(f) \circ \phi_{(X, \Phi)}^{\prime}
$$

for every $f:(X, \Phi) \rightarrow(Y, \Psi)$ in $V_{*}$.

Let $W \in \Phi$ be irreducible. If $\operatorname{dim}(f(W))<\operatorname{dim}(W)$, then $F_{2 \operatorname{dim} W}(Y, f(W))=0$ by semipurity 1.2 .1 ; thus $(1.2 .7)$ holds in this case.

In the case $\operatorname{dim} f(W)=\operatorname{dim} W=: d$, the map $W \rightarrow f(W)$ is generically finite, so that we may find an open $U \subset Y$ such that $U \cap f(W) \neq \varnothing, U \cap f(W)$ is smooth, $f^{-1}(U) \cap W$ is smooth, and $f^{\prime}: f^{-1}(U) \cap W \rightarrow U \cap f(W)$ induced by $f$ is finite. Consider the commutative diagram

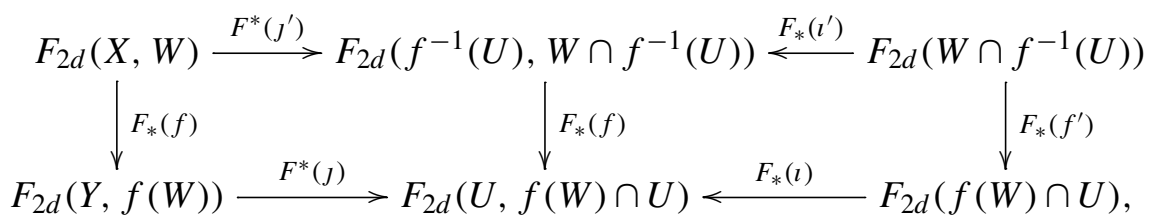

where $J:(U, f(W) \cap U) \rightarrow(Y, f(W))$ and $J^{\prime}:\left(f^{-1}(U), W \cap f^{-1}(U)\right) \rightarrow(X, W)$ in $V^{*}$ are induced by the obvious open immersions; the obvious closed immersions respectively induce $\imath: f(W) \cap U \rightarrow(U, f(W) \cap U)$ and $\iota^{\prime}: W \cap f^{-1}(U) \rightarrow$ $\left(f^{-1}(U), W \cap f^{-1}(U)\right)$ in $V_{*}$. From the diagram and condition 1.2.3(1), we obtain

$$
F^{*}(J) F_{*}(f)\left(c l_{(X, W)}\right)=\operatorname{deg}(W / f(W)) \cdot F_{*}(l)\left(1_{f(W) \cap U}\right) .
$$

Now, semipurity 1.2.1 implies

$$
F_{*}(f)\left(c l_{(X, W)}\right)=\operatorname{deg}(W / f(W)) \cdot c l_{(Y, f(W))},
$$

which proves the claim (1.2.7).

2nd step: Let $X$ be a smooth scheme, $W \subset X$ an irreducible closed subset, and $D \subset X$ a smooth divisor intersecting $W$ properly, so that $W \cap D:=W \times_{X} D$ is an effective Cartier divisor on $W$. We denote by $[W \cap D]$ the associated Weil divisor and claim that

$$
F^{*}\left(l_{D}\right)\left(\phi_{(X, W)}^{\prime}([W])\right)=\phi_{(D, W \cap D)}^{\prime}([W \cap D]),
$$

where $\imath_{D}:(D, W \cap D) \rightarrow(X, W)$ is induced by $D \subset X$. 
Note that by semipurity we may replace $X$ by an open subset that contains the generic points of $(W \cap D)_{\text {red }}$. In particular, we may assume that the irreducible components of $(W \cap D)_{\text {red }}$ are disjoint. Letting $V_{1}, \ldots, V_{r}$ be the irreducible components of $(W \cap D)_{\text {red }}$, we obtain

$$
\bigoplus_{i=1}^{r} F\left(D, V_{i}\right) \stackrel{\cong}{\longrightarrow} F(D, W \cap D)
$$

from 1.1.9(1); thus we may assume that $r=1$. If $W$ is regular (that is, smooth) in codimension one (for example, $W$ is normal), then we can find an open $U \subset X$ such that $W \cap U$ and $V_{1} \cap U \neq \varnothing$ is smooth; thus (1.2.8) follows from 1.2.3(3).

Now, let $W$ be not necessarily normal. Since we may assume that $X$ is affine we can find a closed immersion $\tilde{W} \rightarrow W \times \mathbb{P}^{n}$ (over $W$ ) of the normalization $\tilde{W}$ of $W$. Setting

$$
\tilde{X}:=X \times \mathbb{P}^{n}, \quad \tilde{D}:=D \times \mathbb{P}^{n}, \quad \tilde{\imath}:(\tilde{D}, \tilde{V} \cap \tilde{D}) \rightarrow(\tilde{X}, \tilde{V}),
$$

we obtain

$$
\begin{aligned}
& F^{*}(\imath)\left(\phi_{(X, W)}^{\prime}[W]\right)=F^{*}(\imath) F_{*}\left(\operatorname{pr}_{1}\right)\left(\phi_{(\tilde{X}, \tilde{W})}^{\prime}([\tilde{W}])\right) \quad \text { by }(1.2 .7) \\
& =F_{*}\left(\left.\operatorname{pr}_{1}\right|_{\tilde{D}}\right) F^{*}(\tilde{\imath})\left(\phi_{(\tilde{X}, \tilde{W})}^{\prime}([\tilde{W}])\right) \quad \text { by 1.1.9(4) } \\
& =F_{*}\left(\left.\operatorname{pr}_{1}\right|_{\tilde{D}}\right)\left(\phi_{(\tilde{D}, \tilde{W} \cap \tilde{D})}^{\prime}([\tilde{W} \cap \tilde{D}])\right) \\
& \left.=\phi_{(D, W \cap D)}^{\prime}\left(\operatorname{pr}_{1 *}([\tilde{W} \cap \tilde{D}])\right)\right) \\
& =\phi_{(D, W \cap D)}^{\prime}([W \cap D]) \text {. }
\end{aligned}
$$

3rd step: For all $(X, \Phi)$ we claim that the map $\phi_{(X, \Phi)}^{\prime}$ satisfies

$$
\phi_{(X, \Phi)}^{\prime}\left(\operatorname{Rat}_{\Phi}(X)\right)=0 ;
$$

and thus induces a natural transformation $\phi: \mathrm{CH}_{*} \rightarrow F_{*}$.

Let $W \subset X \times \mathbb{P}^{1}$ be irreducible such that $\operatorname{pr}_{1}(W) \in \Phi$ and $W \rightarrow \mathbb{P}^{1}$ is dominant. By using the 2nd step's (1.2.8), we obtain

$$
F^{*}\left(l_{\epsilon}\right)\left(\phi_{\left(X \times \mathbb{P}^{1}, W\right)}^{\prime}([W])\right)=\phi_{\left(X, \mathrm{pr}_{1}(W)\right)}^{\prime}([W \cap(X \times\{\epsilon\})])
$$

for $\epsilon \in\{0, \infty\}, l_{\epsilon}:\left(X \times\{\epsilon\}, \operatorname{pr}_{1}(W)\right) \rightarrow\left(X \times \mathbb{P}^{1}, W\right)$.

Thus $F^{*}\left(l_{0}\right)=F^{*}\left(l_{\infty}\right)$ will prove the claim (1.2.9). It is not difficult to see that this follows from the projection formula and

$$
F_{*}\left(l_{0}^{\prime}\right)\left(1_{X}\right)=F_{*}\left(l_{\infty}^{\prime}\right)\left(1_{X}\right)
$$

in $F\left(X \times \mathbb{P}^{1}\right)$, where $\imath_{\epsilon}^{\prime}: X \times\{\epsilon\} \stackrel{\subset}{\rightarrow} X \times \mathbb{P}^{1}$.

In view of 1.1.9(4) the equality (1.2.10) is implied by 1.2.3(2). 
4th step: The only assertion left to prove is

$$
\phi \circ \times=T \circ(\phi \otimes \phi), \quad \phi \circ 1=e .
$$

The second equality holds by definition. For the first it suffices to show

$$
\phi_{(X \times Y, W \times V)}^{\prime}([W] \times[V])=T\left(\phi_{(X, W)}^{\prime}([W]) \otimes \phi_{(Y, V)}^{\prime}([V])\right)
$$

for smooth schemes $X, Y$ and irreducible closed subsets $W \subset X, V \subset Y$. Again by semipurity we may assume that $W$ and $V$ are smooth, in which case the statement follows from Lemma 1.1.17.

Proof of Theorem 1.2.3. Set $\mathrm{CH}:=\left(\mathrm{CH}_{*}, \mathrm{CH}^{*}, \times, 1\right)$ and $F:=\left(F_{*}, F^{*}, T, e\right)$. For $\phi \in \operatorname{Hom}_{\mathbf{T}}(\mathrm{CH}, F)$, we get

$$
\phi\left(1_{X}\right)=1_{X}
$$

for all smooth schemes $X$; thus Proposition 1.2.4 implies that $\operatorname{Hom}_{\mathbf{T}}(\mathrm{CH}, F)$ is either empty or contains only one element.

Obviously the conditions (1)-(4) of 1.2.3 are necessary for $\operatorname{Hom}_{\mathbf{T}}(\mathrm{CH}, F)$ to be nonempty. So let us assume that the conditions are satisfied. Proposition 1.2.4 yields a natural transformation of right-lax symmetric monoidal functors

$$
\phi:\left(\mathrm{CH}_{*}, \times, 1\right) \rightarrow\left(F_{*}, T, e\right) .
$$

We need to prove that $\phi$ induces a natural transformation $\phi: \mathrm{CH}^{*} \rightarrow F^{*}$. 1st step: Assume that $f:(X, \Phi) \rightarrow(Y, \Psi)$ in $V^{*}$ is smooth. We claim diagram

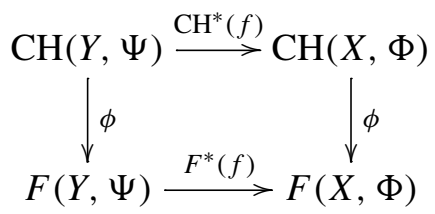

commutes. It suffices to prove

$$
F^{*}(f)\left(\phi_{(Y, V)}([V])\right)=\phi_{\left(X, f^{-1}(V)\right)}\left(f^{*}[V]\right)
$$

for all irreducible closed subsets $V \subset Y$. By using semipurity we may replace $Y$ by an open set and thus assume that $V$ is smooth. We obtain

$$
\begin{aligned}
F^{*}(f)\left(\phi_{(Y, V)}([V])\right) & =F^{*}(f) F_{*}\left(l_{V}\right)\left(1_{V}\right) \\
& =F_{*}\left(l_{f^{-1}(V)}\right) F^{*}\left(\left.f\right|_{f^{-1}(V)}\right)\left(1_{V}\right) \quad \text { by 1.1.9(4) } \\
& =F_{*}\left(l_{f^{-1}(V)}\right)\left(1_{f^{-1}(V)}\right) \\
& =\phi_{\left(X, f^{-1}(V)\right)}\left(\left[f^{-1}(V)\right]\right),
\end{aligned}
$$

where $\iota_{V}: V \rightarrow(Y, V)$ and $\iota_{f^{-1}(V)}: f^{-1}(V) \rightarrow\left(X, f^{-1}(V)\right)$. 
2nd step: Let $p: E \rightarrow X$ be a vector bundle and let $s: X \rightarrow E$ be the zero section. We claim that for every closed subscheme $W \subset X$ the following diagram is commutative:

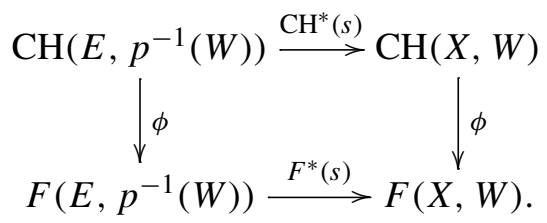

Indeed, by homotopy invariance we may write any $a \in \mathrm{CH}\left(p^{-1}(W)\right)$ as $a=$ $\mathrm{CH}^{*}(p)(b)$ with $b \in \mathrm{CH}(W)$. Thus by the 1 st step,

$$
F^{*}(s)(\phi(a))=F^{*}(s) F^{*}(p)(\phi(b))=\phi(b)=\phi\left(\mathrm{CH}^{*}(s)(a)\right) .
$$

3rd step: For every closed subscheme $W \subset X$, denote by

$$
\iota_{0}:(X, W) \rightarrow\left(X \times \mathbb{P}^{1}, W \times \mathbb{P}^{1}\right) \quad \text { and } \quad \iota_{\infty}:(X, W) \rightarrow\left(X \times \mathbb{P}^{1}, W \times \mathbb{P}^{1}\right),
$$

the morphisms in $V^{*}$ and $V_{*}$ induced by the inclusions $X \times\{0\} \subset X \times \mathbb{P}^{1}$ and $X \times\{\infty\} \subset X \times \mathbb{P}^{1}$, respectively. We claim that

$$
F^{*}\left(l_{0}\right)=F^{*}\left(l_{\infty}\right) .
$$

Indeed, if $p:\left(X \times \mathbb{P}^{1}, W \times \mathbb{P}^{1}\right) \rightarrow(X, W)$ is the first projection, then

$$
F^{*}\left(l_{\epsilon}\right)(a)=F_{*}(p) F_{*}\left(l_{\epsilon}\right)\left(\phi([X]) \cup F^{*}\left(l_{\epsilon}\right)(a)\right)=F_{*}(p)\left(F_{*}\left(l_{\epsilon}\right)(\phi([X])) \cup a\right)
$$

for $\epsilon \in\{0, \infty\}$. Since $F_{*}\left(l_{\epsilon}\right)(\phi([X]))=\phi([X \times\{\epsilon\}])$ the claim follows from $[X \times\{0\}]=[X \times\{\infty\}]$ in $\mathrm{CH}^{1}\left(X \times \mathbb{P}^{1}\right)$.

4th step: Let $f: X \rightarrow Y$ be a closed immersion and $V \subset Y$ a closed subscheme; set $W:=f^{-1}(V)=V \times_{Y} X$. Then $f$ induces $f:(X, W) \rightarrow(Y, V)$ in $V^{*}$ and we claim that

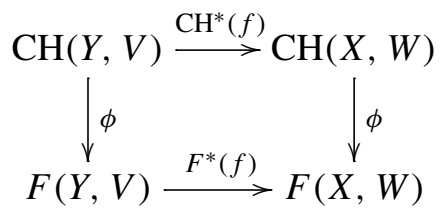

is a commutative diagram.

Again, it is sufficient to prove

$$
F^{*}(f)(\phi([V]))=\phi\left(\mathrm{CH}^{*}(f)\right)
$$

for $V$ integral. 
For the proof we use deformation to the normal cone [Fulton 1998, Section 5]. Let

$$
\begin{aligned}
& M^{0}:=\mathrm{Bl}_{X \times\{\infty\}}\left(Y \times \mathbb{P}^{1}\right) \backslash \mathrm{B}_{X \times\{\infty\}}(Y \times\{\infty\}), \\
& \tilde{M}^{0}:=\mathrm{Bl}_{W \times\{\infty\}}\left(V \times \mathbb{P}^{1}\right) \backslash \mathrm{B}_{W \times\{\infty\}}(V \times\{\infty\}) ;
\end{aligned}
$$

then $\tilde{M}^{0} \subset M^{0}$ is closed, and $M^{0}$ and $\tilde{M}^{0}$ are flat over $\mathbb{P}^{1}$. We have closed immersions $\iota_{X}: X \times \mathbb{P}^{1} \rightarrow M^{0}$ and $\iota_{W}: W \times \mathbb{P}^{1} \rightarrow \tilde{M}^{0}$ that deform the immersions $X \subset Y$ and $W \subset V$, respectively, over $\mathbb{P}^{1} \backslash\{\infty\}$ to the zero section of the normal cone over $\infty$.

Since $W \times \mathbb{P}^{1}=\tilde{M}^{0} \cap\left(X \times \mathbb{P}^{1}\right)$ we obtain morphisms

$$
l_{\epsilon}:(X \times\{\epsilon\}, W \times\{\epsilon\}) \rightarrow\left(M^{0}, \tilde{M}^{0}\right)
$$

in $V^{*}$ for $\epsilon \in\{0, \infty\}$. By the 3rd step we know that $F^{*}\left(l_{0}\right)=F^{*}\left(l_{\infty}\right)$.

Consider the projection $p:\left(Y \times\left(\mathbb{P}^{1} \backslash\{\infty\}\right), V \times\left(\mathbb{P}^{1} \backslash\{\infty\}\right)\right) \rightarrow(Y, V)$ in $V^{*}$. Note that $\tilde{M}^{0}$ is the closure of $V \times\left(\mathbb{P}^{1} \backslash\{\infty\}\right)$ in $M^{0}$, and thus

$$
\mathrm{CH}^{*}(p)([V])=\mathrm{CH}^{*}(J)\left(\left[\tilde{M}^{0}\right]\right)
$$

with $J:\left(Y \times\left(\mathbb{P}^{1} \backslash\{\infty\}\right), V \times\left(\mathbb{P}^{1} \backslash\{\infty\}\right)\right) \rightarrow\left(M^{0}, \tilde{M}^{0}\right)$ the open immersion. By using the 1st step we get $F^{*}(p)(\phi([V]))=F^{*}(\jmath)\left(\phi\left(\left[\tilde{M}^{0}\right]\right)\right)$ and thus

$$
F^{*}(f)(\phi([V]))=F^{*}\left(l_{0}\right)\left(\phi\left(\left[\tilde{M}^{0}\right]\right)\right)=F^{*}\left(l_{\infty}\right)\left(\phi\left(\left[\tilde{M}^{0}\right]\right)\right) .
$$

Now, let us compute $F^{*}\left(l_{\infty}\right)$. The morphism $l_{\infty}$ has a factorization

$$
l_{\infty}:(X, W) \stackrel{s}{\rightarrow}\left(N_{Y / X}, C_{V / W}\right) \stackrel{t}{\rightarrow}\left(M^{0}, \tilde{M}^{0}\right),
$$

where $N_{Y / X}$ is the normal bundle and $C_{V / W}$ is the normal cone. Note that $N_{Y / X}$ is a smooth divisor in $M^{0}$, which intersects $\tilde{M}^{0}$ properly (being the fiber of $M^{0} \rightarrow \mathbb{P}^{1}$ over $\infty$ ), so that we may apply (1.2.8) to $t$. Moreover $s$ is the zero section of the normal bundle. The zero section also induces a morphism

$$
s^{\prime}:(X, W) \rightarrow\left(N_{Y / X}, N_{Y / X} \times_{X} W\right) \text { in } V^{*} ;
$$

denote by $\tau:\left(N_{Y / X}, C_{V / W}\right) \rightarrow\left(N_{Y / X}, N_{Y / X} \times_{X} W\right)$ the morphism in $V_{*}$ induced by the identity map. Then 1.1.9(4) yields

$$
F^{*}(s)=F^{*}\left(s^{\prime}\right) \circ F_{*}(\tau) .
$$

Thus we get

$$
\begin{array}{rlrl}
F^{*}\left(l_{\infty}\right)\left(\phi\left(\left[\tilde{M}^{0}\right]\right)\right) & =F^{*}\left(s^{\prime}\right) F_{*}(\tau) F^{*}(t)\left(\phi\left(\left[\tilde{M}^{0}\right]\right)\right) & \\
& =F^{*}\left(s^{\prime}\right) F_{*}(\tau)\left(\phi\left(\mathrm{CH}^{*}(t)\left(\left[\tilde{M}^{0}\right]\right)\right)\right) & & \text { by }(1.2 .8) \\
& =\phi\left(\mathrm{CH}^{*}\left(l_{\infty}\right)\left(\left[\tilde{M}^{0}\right]\right)\right) & & \text { by the 2nd step } \\
& =\phi\left(\mathrm{CH}^{*}\left(l_{0}\right)\left(\left[\tilde{M}^{0}\right]\right)\right)=\phi\left(\mathrm{CH}^{*}(f)([V])\right) .
\end{array}
$$


5th step: Let $f:(X, \Phi) \rightarrow(Y, \Psi)$ be any morphism in $V^{*}$. We have to prove that

$$
\phi \circ \mathrm{CH}^{*}(f)=F^{*}(f) \circ \phi .
$$

Indeed, $f$ factors through

$$
f:(X, \Phi) \stackrel{(\mathrm{id}, f)}{\longrightarrow}\left(X \times Y, \mathrm{pr}_{2}^{-1}(\Psi)\right) \stackrel{\mathrm{pr}_{2}}{\longrightarrow}(Y, \Psi) .
$$

By the 1st step we may reduce to the case of the closed immersion (id, $f$ ), and by using Proposition 1.1.29 the statement follows from the 4th step.

1.3. Correspondences. Let $\left(F_{*}, F^{*}, T, e\right) \in \mathbf{T}$. Let $X_{i}$ for $i=1,2,3$ be smooth varieties and $\Phi_{i j}$ for $i j=12,23,13$ be families of supports on $X_{i} \times X_{j}$. Denote by $p_{i j}: X_{1} \times X_{2} \times X_{3} \rightarrow X_{i} \times X_{j}$ the projection. Suppose that

$$
\left\{\begin{array}{l}
\left.p_{13}\right|_{p_{12}^{-1}\left(\Phi_{12}\right) \cap p_{23}^{-1}\left(\Phi_{23}\right)} \text { is proper, } \\
p_{13}\left(p_{12}^{-1}\left(\Phi_{12}\right) \cap p_{23}^{-1}\left(\Phi_{23}\right)\right) \subset \Phi_{13}
\end{array}\right.
$$

Then we define

$$
F\left(X_{1} \times X_{2}, \Phi_{12}\right) \otimes F\left(X_{2} \times X_{3}, \Phi_{23}\right) \rightarrow F\left(X_{1} \times X_{3}, \Phi_{13}\right), \quad a \otimes b \mapsto b \circ a
$$

to be the composition

$$
\begin{aligned}
F & \left(X_{1} \times X_{2}, \Phi_{12}\right) \otimes F\left(X_{2} \times X_{3}, \Phi_{23}\right) \\
& \stackrel{F^{*}\left(p_{12}\right) \otimes F^{*}\left(p_{23}\right)}{\longrightarrow} F\left(X_{1} \times X_{2} \times X_{3}, p_{12}^{-1}\left(\Phi_{12}\right)\right) \otimes F\left(X_{1} \times X_{2} \times X_{3}, p_{23}^{-1}\left(\Phi_{23}\right)\right) \\
& \stackrel{\cup}{\longrightarrow} F\left(X_{1} \times X_{2} \times X_{3}, p_{12}^{-1}\left(\Phi_{12}\right) \cap p_{23}^{-1}\left(\Phi_{23}\right)\right) \\
& \stackrel{F_{*}\left(p_{13}\right)}{\longrightarrow} F\left(X_{1} \times X_{3}, \Phi_{13}\right) .
\end{aligned}
$$

1.3.3. Let $\Phi_{i j}^{\prime}$ for $i j=12,23,13$ be families of supports on $X_{i} \times X_{j}$. Suppose that

$$
\left\{\begin{array}{l}
\left.p_{13}\right|_{p_{12}^{-1}\left(\Phi_{12}^{\prime}\right) \cap p_{23}^{-1}\left(\Phi_{23}^{\prime}\right)} \text { is proper, } \\
p_{13}\left(p_{12}^{-1}\left(\Phi_{12}^{\prime}\right) \cap p_{23}^{-1}\left(\Phi_{23}^{\prime}\right)\right) \subset \Phi_{13}^{\prime},
\end{array}\right.
$$

and $\Phi_{i j}^{\prime} \subset \Phi_{i j}$ for $i j=12,23,13$. Obviously, the diagram

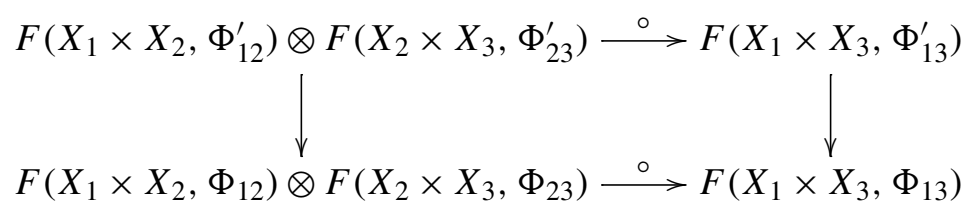

is commutative.

The most important case for us will be $\left(\mathrm{CH}_{*}, \mathrm{CH}^{*}, \times, 1\right)$. For later use we record the following particular case of the discussion above. 
Lemma 1.3.4. Let $X_{i}$ for $i=1,2,3$ be smooth schemes and $\Phi_{i j}$ for $i j=12,23,13$ be families of supports on $X_{i} \times X_{j}$ that satisfy (1.3.1). For $a \in Z_{\Phi_{12}}\left(X_{1} \times X_{2}\right)$ and $b \in Z_{\Phi_{23}}\left(X_{2} \times X_{3}\right)$, we define

$$
\operatorname{supp}(a, b):=p_{13}\left(p_{12}^{-1}(\operatorname{supp}(a)) \cap p_{23}^{-1}(\operatorname{supp}(b))\right),
$$

which is a closed subset contained in $\Phi_{13}$. The families of supports

$$
\Phi_{12}^{\prime}=\Phi_{\operatorname{supp}(a)}, \quad \Phi_{23}^{\prime}=\Phi_{\operatorname{supp}(b)}, \quad \Phi_{13}^{\prime}=\Phi_{\operatorname{supp}(a, b)}
$$

satisfy (1.3.1). The cycles $a$ and $b$ define in the obvious way classes

$$
\begin{array}{lll}
\tilde{a} \in \mathrm{CH}(\operatorname{supp}(a)), & \tilde{b} \in \mathrm{CH}(\operatorname{supp}(b)), \\
a \in \mathrm{CH}\left(X_{1} \times X_{2}, \Phi_{12}\right), & b \in \mathrm{CH}\left(X_{2} \times X_{3}, \Phi_{23}\right) .
\end{array}
$$

Then $b \circ a$ is the image of $\tilde{b} \circ \tilde{a}$ via the map $\mathrm{CH}(\operatorname{supp}(a, b)) \rightarrow \mathrm{CH}\left(X_{1} \times X_{3}, \Phi_{13}\right)$.

Lemma 1.3.4 helps to understand the composition of two cycles $a$ and $b$ via the purely set-theoretic computation of $\operatorname{supp}(a, b)$. Frequently we are able to compute the composition over suitable good open subsets; this is the motivation for the next lemma.

Lemma 1.3.6. Let $X_{i}$ for $i=1,2,3$ be smooth schemes. Let $a \in Z\left(X_{1} \times X_{2}\right)$ and $b \in Z\left(X_{2} \times X_{3}\right)$ be algebraic cycles such that

$$
\left.p_{13}\right|_{p_{12}^{-1} \operatorname{supp}(a) \cap p_{23}^{-1} \operatorname{supp}(b)} \text { is proper. }
$$

Let $X_{1}^{\prime} \subset X_{1}, X_{3}^{\prime} \subset X_{3}$ be open subsets; define $a^{\prime} \in Z\left(X_{1}^{\prime} \times X_{2}\right), b^{\prime} \in Z\left(X_{2} \times X_{3}^{\prime}\right)$ as the restrictions of $a, b$. We denote by $p_{i j}^{\prime}$ the projections from $X_{1}^{\prime} \times X_{2} \times X_{3}^{\prime}$.

(1) The restriction of $p_{13}^{\prime}$ to $p_{12}^{\prime-1} \operatorname{supp}\left(a^{\prime}\right) \cap p_{23}^{\prime-1} \operatorname{supp}\left(b^{\prime}\right)$ is proper.

(2) The equality

$$
\operatorname{supp}\left(a^{\prime}, b^{\prime}\right)=\operatorname{supp}(a, b) \cap\left(X_{1}^{\prime} \times X_{3}^{\prime}\right)
$$

holds, where $\operatorname{supp}(a, b)$ is defined in (1.3.5).

(3) The composition $b^{\prime} \circ a^{\prime}$ is the image of $b \circ a$ via the localization map

$$
\mathrm{CH}(\operatorname{supp}(a, b)) \rightarrow \mathrm{CH}\left(\operatorname{supp}\left(a^{\prime}, b^{\prime}\right)\right) .
$$

$\left(\right.$ Here $\operatorname{supp}\left(a^{\prime}, b^{\prime}\right) \subset \operatorname{supp}(a, b)$ is an open subset by $\left.(2)\right)$.

Proof. By definition we obtain

$$
\operatorname{supp}\left(a^{\prime}\right)=\operatorname{supp}(a) \cap\left(X_{1}^{\prime} \times X_{2}\right), \quad \operatorname{supp}\left(b^{\prime}\right)=\operatorname{supp}(b) \cap\left(X_{2} \times X_{3}^{\prime}\right) .
$$

For (1): Let $Z_{12} \subset X_{1} \times X_{2}, Z_{23} \subset X_{2} \times X_{3}$ be closed subsets such that

$$
\left.p_{13}\right|_{p_{12}^{-1} Z_{12} \cap p_{23}^{-1} Z_{23}} \text { is proper. }
$$


Set $Z_{12}^{\prime}=Z_{12} \cap\left(X_{1}^{\prime} \times X_{2}\right)$ and $Z_{23}^{\prime}=Z_{23} \cap\left(X_{2} \times X_{3}^{\prime}\right)$. Obviously,

$$
p_{12}^{\prime-1} Z_{12}^{\prime} \cap p_{23}^{\prime-1} Z_{23}^{\prime}=\left(p_{12}^{-1} Z_{12} \cap p_{23}^{-1} Z_{23}\right) \cap\left(X_{1}^{\prime} \times X_{2} \times X_{3}^{\prime}\right) .
$$

Thus, if $p_{12}^{-1} Z_{12} \cap p_{23}^{-1} Z_{23}$ is proper over $X_{1} \times X_{3}$, then $p_{12}^{\prime-1} Z_{12}^{\prime} \cap p_{23}^{\prime-1} Z_{23}^{\prime}$ is proper over $X_{1}^{\prime} \times X_{3}^{\prime}$.

Statement (2) is a straightforward computation. For (3): By using the definition of $\circ$ in (1.3.2) it is straightforward to show that the diagram

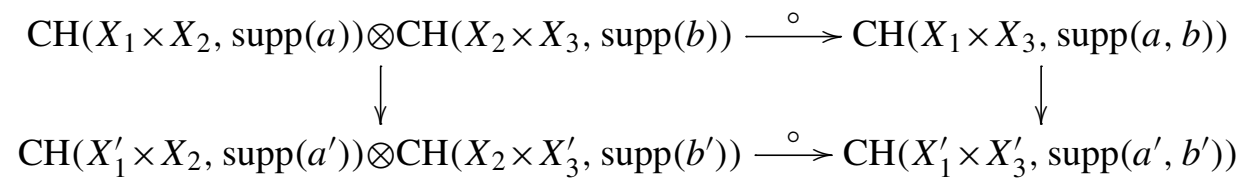

is commutative.

1.3.7. For two smooth schemes $X$ and $Y$ and families $\Phi$ and $\Psi$ of supports of $X$ and $Y$, respectively, we define a family of supports $P(\Phi, \Psi)$ on the product by $P(\Phi, \Psi):=\left\{Z \subset X \times Y ; Z\right.$ is closed, $\mathrm{pr}_{2} \mid Z$ is proper,

$$
\left.Z \cap \operatorname{pr}_{1}^{-1}(W) \in \operatorname{pr}_{2}^{-1}(\Psi) \text { for every } W \in \Phi\right\} .
$$

Let $X_{i}$ for $i=1,2,3$ be smooth schemes and let $\Phi_{i}$ be a family of supports on $X_{i}$ for $i=1,2,3$. It is easy to see that $\Phi_{i j}:=P\left(\Phi_{i}, \Phi_{j}\right)$ satisfy the condition (1.3.1) and therefore

$$
\begin{aligned}
F\left(X_{1} \times X_{2}, P\left(\Phi_{1}, \Phi_{2}\right)\right) \otimes F\left(X_{2} \times X_{3}, P\left(\Phi_{2}, \Phi_{3}\right)\right) & \\
& \rightarrow F\left(X_{1} \times X_{3}, P\left(\Phi_{1}, \Phi_{3}\right)\right),
\end{aligned}
$$

where $a \otimes b \mapsto b \circ a$, is well defined.

Proposition 1.3.10. (1) Let $X_{i}$ for $i=1, \ldots, 4$ be a smooth scheme and let $\Phi_{i}$ for $i=1, \ldots, 4$ be a family of supports of $X_{i}$. We have

$$
a_{34} \circ\left(a_{23} \circ a_{12}\right)=\left(a_{34} \circ a_{23}\right) \circ a_{12} \quad \text { for all } a_{i j} \in F\left(X_{i} \times X_{j}, P\left(\Phi_{i}, \Phi_{j}\right)\right) \text {. }
$$

(2) For any $(X, \Phi)$, the diagonal immersion induces a morphism

$$
\imath: X \rightarrow(X \times X, P(\Phi, \Phi)) \text { in } V_{*} .
$$

We set $\Delta_{(X, \Phi)}:=F_{*}(l)\left(1_{X}\right)$. The equality $\Delta_{(X, \Phi)} \circ g=g$ holds for all $(Y, \Psi)$ and $g \in F(Y \times X, P(\Psi, \Phi))$, and $g \circ \Delta_{(X, \Phi)}=g$ holds for all $(Y, \Psi)$ and $g \in F(X \times Y, P(\Phi, \Psi))$.

Proof. The proof of the first statement is as in [Fulton 1998, Proposition 16.1.1] but one has to keep track of the supports, which is straightforward.

The second statement is an easy computation. 
1.3.11. Grading. For $(X, \Phi)$ and $(Y, \Psi), \quad F_{*}$ and $F^{*}$ give rise to two different gradings on $F(X \times Y, P(\Phi, \Psi))$. Unfortunately, neither are compatible with the $\circ$ from (1.3.9). We define a new grading by

$$
F(X \times Y, P(\Phi, \Psi))^{i}=\bigoplus_{X^{\prime}} F^{2 \operatorname{dim}\left(X^{\prime}\right)+i}\left(X^{\prime} \times Y, P(\Phi, \Psi)\right),
$$

where $X^{\prime}$ runs through the connected components of $X$. With this grading, o becomes a morphism of graded abelian groups.

By the definition of the grading there are choices. We could also define a grading

$$
F(X \times Y, P(\Phi, \Psi))_{i}=\bigoplus_{X^{\prime}} F_{2 \operatorname{dim}\left(X^{\prime}\right)+i}\left(X^{\prime} \times Y, P(\Phi, \Psi)\right) .
$$

Definition 1.3.12. To an object $F=\left(F_{*}, F^{*}, T, e\right) \in \mathbf{T}$, we attach the graded additive symmetric monoidal category $\operatorname{Cor}_{F}$ with objects $\mathrm{ob}\left(\operatorname{Cor}_{F}\right)=\mathrm{ob}\left(V_{*}\right)=$ $\mathrm{ob}\left(V^{*}\right)$ and morphisms

$$
\operatorname{Hom}_{\mathrm{Cor}_{F}}((X, \Phi),(Y, \Psi))=F(X \times Y, P(\Phi, \Psi))
$$

with composition law $a \otimes b \mapsto b \circ a$ (1.3.9). The identity is $\Delta_{(X, \Phi)}$.

The product $\otimes$ on $\operatorname{Cor}_{F}$ is defined by

$$
(X, \Phi) \otimes(Y, \Psi):=(X \times Y, \Phi \times \Psi),
$$

and for two morphisms $f \in F\left(X \times X^{\prime}, P\left(\Phi, \Phi^{\prime}\right)\right)$ and $g \in F\left(Y \times Y^{\prime}, P\left(\Psi, \Psi^{\prime}\right)\right)$, we define

$$
\begin{aligned}
& f \otimes g \in \operatorname{Hom}_{\mathrm{Cor}_{F}}\left((X, \Phi) \otimes(Y, \Psi),\left(X^{\prime}, \Phi^{\prime}\right) \otimes\left(Y^{\prime}, \Psi^{\prime}\right)\right), \\
& f \otimes g:=F_{*}\left(\operatorname{id}_{X} \times \mu_{X^{\prime}, Y} \times \operatorname{id}_{Y^{\prime}}\right)(T(f \otimes g)),
\end{aligned}
$$

where $\mu_{X^{\prime}, Y}$ is the permutation of the factors $\left(X^{\prime}, \Phi^{\prime}\right)$ and $(Y, \Psi)$.

1.3.13. Given two objects $F, G \in \mathbf{T}$ and a morphism $\phi: F \rightarrow G$ in $\mathbf{T}$, we obtain a functor of graded additive symmetric monoidal categories

$$
\operatorname{Cor}(\phi): \operatorname{Cor}_{F} \rightarrow \operatorname{Cor}_{G}
$$

that is given by

$$
\phi: F(X \times Y, P(\Phi, \Psi)) \rightarrow G(X \times Y, P(\Phi, \Psi))
$$

for all $(X, \Phi)$ and $(Y, \Psi)$. This provides a functor

$$
\text { Cor : T } \rightarrow \mathbf{C a t}_{\mathbf{G r A b}, \otimes}, \quad F \mapsto \operatorname{Cor}_{F}, \quad \phi \mapsto \operatorname{Cor}(\phi) .
$$

Here, $\mathbf{C a t}_{\mathbf{G r A b}, \otimes}$ is the category of graded additive symmetric monoidal categories. 
1.3.14. In order to state the properties of Cor, it is convenient to introduce the category $V$ with objects $\mathrm{ob}(V)=\mathrm{ob}\left(V_{*}\right)=\mathrm{ob}\left(V^{*}\right)$ and only morphisms the identity $\operatorname{id}_{X}$ (for every $X \in \mathrm{ob}(V)$ ). There are obvious functors $V \rightarrow V_{*}, V \rightarrow V^{*}$, and $V \rightarrow \operatorname{Cor}_{F}$ for all $F \in \mathbf{T}$. We define $\mathbf{C a t}_{V / \mathbf{G r A b}, \otimes}$ to be the category with functors $V \rightarrow X$ as objects $\left(X \in \mathbf{C a t}_{\mathbf{G r A b}, \otimes}\right)$ and commutative diagrams

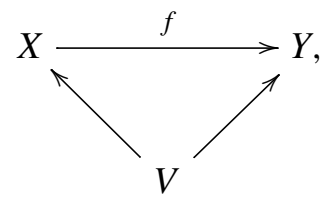

with $f \in \operatorname{Hom}_{\mathbf{C a t}_{\mathbf{G r A b}, \otimes}}(X, Y)$, as morphisms. In general a functor $f$ is said to be under $V$ if the diagram (1.3.15) is commutative.

Proposition 1.3.16. The functor Cor : $\mathbf{T} \rightarrow \mathbf{C a t}_{V / \mathbf{G r A b}, \otimes}$ is fully faithful.

Proof. Given $F, G \in \mathbf{T}$ and $\phi: F \rightarrow G$, we can recover $\phi: F(X) \rightarrow G(X)$ for $X \in \mathrm{ob}(V)$ from the map $\operatorname{Cor}(\phi)$ :

$$
\operatorname{Hom}_{\operatorname{Cor}_{F}}(\operatorname{Spec}(k), X) \rightarrow \operatorname{Hom}_{\operatorname{Cor}_{G}}(\operatorname{Spec}(k), X) .
$$

On the other hand, given $\psi: \operatorname{Cor}_{F} \rightarrow \operatorname{Cor}_{G}$ in $\mathbf{C a t}_{V / \mathbf{G r A b}, \otimes}$, the map (1.3.17) defines a morphism $F \rightarrow G$ in $\mathbf{T}$.

1.3.18. For all $F \in \mathbf{T}$, there is a functor

$$
\rho_{F}: \operatorname{Cor}_{F} \rightarrow \mathbf{G r A b}
$$

defined by

$$
\begin{aligned}
\rho_{F}(X, \Phi) & =F(X, \Phi) \\
\rho_{F}(\gamma) & =\left(a \mapsto F_{*}\left(\operatorname{pr}_{2}\right)\left(F^{*}\left(\operatorname{pr}_{1}\right)(a) \cup \gamma\right)\right) \quad \text { for } \gamma \in F(X \times Y, P(\Phi, \Psi)) .
\end{aligned}
$$

The map $\rho_{F}(\gamma): F(X, \Phi) \rightarrow F(Y, \Psi)$ is well defined since $\mathrm{pr}_{2}$ restricted to $\operatorname{pr}_{1}^{-1}(\Phi) \cap P(\Phi, \Psi)$ is proper and $\operatorname{pr}_{1}^{-1}(\Phi) \cap P(\Phi, \Psi) \subset \operatorname{pr}_{2}^{-1}(\Psi)$ by definition of $P(\Phi, \Psi)$. Functoriality is again a straightforward computation.

Moreover, there are functors

$$
\tau_{*}^{F}: V_{*} \rightarrow \operatorname{Cor}_{F} \quad \text { and } \quad \tau_{F}^{*}:\left(V^{*}\right)^{\mathrm{op}} \rightarrow \operatorname{Cor}_{F},
$$

(under $V$ ) such that

$$
\rho_{F} \circ \tau_{*}^{F}=F_{*} \quad \text { and } \quad \rho_{F} \circ \tau_{F}^{*}=F^{*} .
$$

The functor $\tau_{*}^{F}: V_{*} \rightarrow \operatorname{Cor}_{F}$ is defined by mapping a morphism $f:(X, \Phi) \rightarrow(Y, \Psi)$ to $F_{*}(\mathrm{id}, f)\left(1_{X}\right)$, where (id, $\left.f\right): X \rightarrow(X \times Y, P(\Phi, \Psi))$ is in $V_{*}$. Similarly, the functor $\tau_{F}^{*}: V^{*} \rightarrow \operatorname{Cor}_{F}$ is defined by mapping a morphism $f:(X, \Phi) \rightarrow(Y, \Psi)$ 
to $F_{*}(f$, id $)\left(1_{X}\right)$ with $(f$, id $): X \rightarrow(Y \times X, P(\Psi, \Phi))$ in $V_{*}$. Then the equalities $\rho_{F} \circ \tau_{*}^{F}=F_{*}$ and $\rho_{F} \circ \tau_{F}^{*}=F^{*}$ follow easily from the projection formula.

Lemma 1.3.19. If $\phi: F \rightarrow G$ is a morphism in $\mathbf{T}$, then

$$
\operatorname{Cor}(\phi) \circ \tau_{*}^{F}=\tau_{*}^{G} \quad \text { and } \quad \operatorname{Cor}(\phi) \circ \tau_{F}^{*}=\tau_{G}^{*} .
$$

Proof. For the first equality, let $f:(X, \Phi) \rightarrow(Y, \Psi)$ be a morphism in $V_{*}$. We get

$$
\begin{aligned}
\operatorname{Cor}(\phi)\left(\tau_{*}^{F}(f)\right) & =\operatorname{Cor}(\phi)\left(F_{*}(\mathrm{id}, f)\left(1_{X}\right)\right)=\phi\left(F_{*}(\mathrm{id}, f)\left(1_{X}\right)\right) \\
& =G_{*}(\mathrm{id}, f)\left(\phi\left(1_{X}\right)\right)=G_{*}(\mathrm{id}, f)\left(1_{X}\right)=\tau_{*}^{G}(f) .
\end{aligned}
$$

The second equality is proved in the same way.

\section{Hodge cohomology with support}

For a smooth scheme $X$ and a family of supports $\Phi$ of $X$, we define

$$
H(X, \Phi):=\bigoplus_{i, j} H_{\Phi}^{i}\left(X, \Omega_{X}^{j}\right)
$$

and call this $k$-vector space the Hodge cohomology of $X$ with support in $\Phi$. We denote by $H^{*}(X, \Phi)$ the graded abelian group, which in degree $n$ equals

$$
H^{n}(X, \Phi)=\bigoplus_{i+j=n} H_{\Phi}^{i}\left(X, \Omega_{X}^{j}\right)
$$

We denote by $H_{*}(X, \Phi)$ the graded abelian group, which in degree $n$ equals

$$
H_{n}(X, \Phi)=\bigoplus_{r} H^{2 \operatorname{dim} X_{r}-n}\left(X_{r}, \Phi\right),
$$

where $X=\bigsqcup_{r} X_{r}$ is the decomposition into connected components. We define

$$
e: \mathbb{Z} \rightarrow H(\operatorname{Spec} k)=k
$$

to be the natural map sending 1 to 1 .

The goal of this section is to provide the object functions $H_{*}$ and $H^{*}$ with the structure of functors

$$
H_{*}: V_{*} \rightarrow \mathbf{G r A b} \text { and } H^{*}:\left(V^{*}\right)^{\mathrm{op}} \rightarrow \mathbf{G r A b}
$$

and to define for each $(X, \Phi),(Y, \Psi) \in \mathrm{ob}\left(V_{*}\right)=\mathrm{ob}\left(V^{*}\right)$ a morphism

$$
T_{(X, \Phi),(Y, \Psi)}: H(X, \Phi) \otimes H(Y, \Psi) \rightarrow H(X \times Y, \Phi \times \Psi)
$$

of graded abelian groups (for both gradings) such that $\left(H_{*}, H^{*}, T, e\right)$ is an object in $\mathbf{T}$, that is, it is a datum as in 1.1.8 and satisfies the properties 1.1.9.

\subsection{Pullback.}


2.1.1. We work in the bounded derived category of quasicoherent sheaves $D^{b}(X)$ on a scheme $X$. (The bounded derived category of coherent sheaves will be denoted by $D_{c}^{b}(X)$.) Let $f: X \rightarrow Y$ be a morphism of schemes; let $\Phi$ and $\Psi$ be families of supports of $X$ and $Y$, respectively. There is an isomorphism of functors

$$
R \Gamma_{f^{-1}(\Psi)} \stackrel{\cong}{\rightrightarrows} R \Gamma_{\Psi} R f_{*} .
$$

If $\Psi \subset \Psi^{\prime}$ for another family of supports $\Psi^{\prime}$, then the diagram

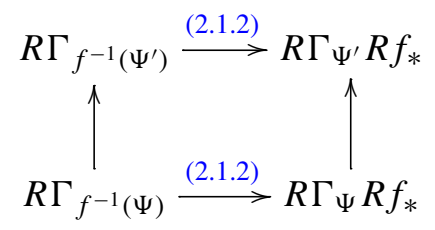

is commutative. Moreover, if $g: Z \rightarrow X$ is another morphism of schemes then the following diagram is commutative:

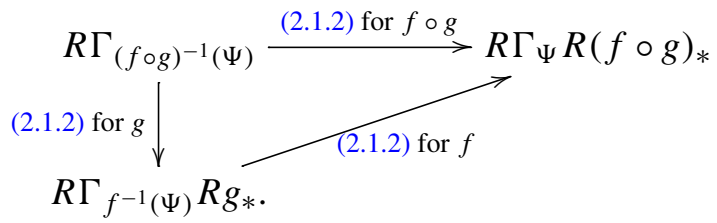

2.1.5. For a morphism $f: X \rightarrow Y$ of schemes, we have

$$
\mathrm{id} \rightarrow R f_{*} L f^{*}
$$

and thus we obtain a morphism of functors

$$
R \Gamma_{\Psi} \rightarrow R \Gamma_{f^{-1}(\Psi)} L f^{*}
$$

it easily follows from (2.1.3) that the diagram

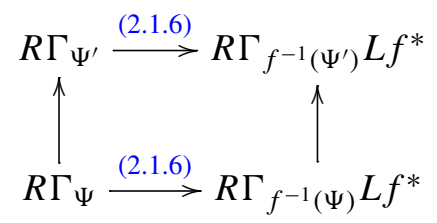

commutes for $\Psi \subset \Psi^{\prime}$. From (2.1.7) and (2.1.4) it follows that for another morphism $g: Z \rightarrow X$ of schemes the following diagram is commutative:

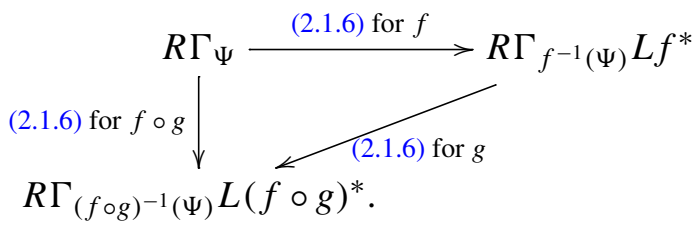


For a morphism $f:(X, \Phi) \rightarrow(Y, \Psi)$ in $V^{*}$ (that is, $\left.f^{-1}(\Psi) \subset \Phi\right)$, the morphism

$$
R \Gamma_{\Psi} \Omega_{Y}^{d} \rightarrow R \Gamma_{f^{-1}(\Psi)} L f^{*} \Omega_{Y}^{d}=R \Gamma_{f^{-1}(\Psi)} f^{*} \Omega_{Y}^{d} \rightarrow R \Gamma_{f^{-1}(\Psi)} \Omega_{X}^{d} \rightarrow R \Gamma_{\Phi} \Omega_{X}^{d}
$$

(for $d \geq 0$ ) gives a morphism

$$
H^{*}(f): H(Y, \Psi) \rightarrow H(X, \Phi) .
$$

By a straightforward computation, $f \mapsto H^{*}(f)$ defines a functor $\left(V^{*}\right)^{\mathrm{op}} \rightarrow \mathbf{G r A b}$.

2.2. Push-forward in the derived category. We recall the following notations from duality theory [Hartshorne 1966; Conrad 2000]: Let $X$ be a separated $k$-scheme of finite type with structure map $\pi: X \rightarrow$ Spec $k$. We have $\pi^{!} k \in D_{c}^{b}(X)$. (In fact if $X$ has dimension $d$, then $\pi ! k$ has nonzero cohomology only in the interval $[-d, 0]$. This follows from [Hartshorne 1966, Chapter V, Proposition 7.3 and its proof] and [Conrad 2000, (3.1.25)].) We denote

$$
D_{X}:=R \operatorname{Hom}_{X}\left(\cdot, \pi^{!} k\right): D_{c}^{b}(X) \rightarrow D_{c}^{b}(X) .
$$

If $f: X \rightarrow Y$ is a proper morphism between $k$-schemes, we have the trace map

$$
\operatorname{Tr}_{f}: R f_{*} f^{!} \rightarrow \mathrm{id}
$$

which is a natural transformation of functors on $D_{c}^{+}(Y)$. For maps $f: X \rightarrow Y$ and $g: Y \rightarrow Z$, we have the canonical isomorphisms

$$
c_{f, g}:(g f)^{!} \stackrel{\simeq}{\longrightarrow} f^{!} g^{!} \quad \text { in } D_{c}^{+}(X) .
$$

Notation 2.2.4. Given a bounded complex $C$ in $D(X)$ and a morphism of complexes $\varphi: A \rightarrow B$ in $D(X)$, we will often denote the morphism $R \mathscr{H} \operatorname{om}_{X}(C, \varphi)$ : $R \mathscr{H}_{X}(C, A) \rightarrow R \mathscr{H} \operatorname{om}_{X}(C, B)$ simply by $\varphi$ and the morphism $R \mathscr{H}_{X}(\varphi, C):$ $R \mathscr{H} \operatorname{om}_{X}(B, C) \rightarrow R \mathscr{H} \operatorname{om}_{X}(A, C)$ by $\varphi^{\vee}$. It will always be clear from the context what $C$ is in the particular situation.

Definition 2.2.5. Let $f: X \rightarrow Y$ be a proper $k$-morphism. Let $\pi_{X}$ and $\pi_{Y}$ denote the structure maps of $X$ and $Y$ respectively. Then we define

$$
f_{*}: R f_{*} D_{X}\left(\Omega_{X}^{q}\right) \rightarrow D_{Y}\left(\Omega_{Y}^{q}\right) \text { for } q \geq 0,
$$

to be the composition

$$
\begin{aligned}
& R f_{*} R \operatorname{Hom}_{X}\left(\Omega_{X}^{q}, \pi_{X}^{!} k\right) \stackrel{c_{f, \pi_{Y}}}{\longrightarrow} R f_{*} R \operatorname{Hom}_{X}\left(\Omega_{X}^{q}, f^{!} \pi_{Y}^{!} k\right) \\
& \stackrel{\text { nat. }}{\longrightarrow} R \mathcal{H} \operatorname{om}_{Y}\left(R f_{*} \Omega_{X}^{q}, R f_{*} f^{!} \pi_{Y}^{!} k\right) \stackrel{\operatorname{Tr}_{f}}{\longrightarrow} R \mathcal{H} \operatorname{om}_{Y}\left(R f_{*} \Omega_{X}^{q}, \pi_{Y}^{!} k\right) \\
& \stackrel{\left(f^{*}\right)^{\vee}}{\longrightarrow} R \operatorname{Hom}_{Y}\left(\Omega_{Y}^{q}, \pi_{Y}^{!} k\right) .
\end{aligned}
$$


Remark 2.2.6. (1) Notice that the composition of the middle two arrows in the composition above is just the standard Grothendieck duality isomorphism (see for example [Conrad 2000, (3.4.10)])

$$
R f_{*} R \operatorname{Hom}_{X}\left(\cdot, f^{!}(\cdot)\right) \stackrel{\simeq}{\rightarrow} R \operatorname{Hom}_{Y}\left(R f_{*}(\cdot), \cdot\right) .
$$

(2) It is straightforward to check that the push-forward above also equals the composition

$$
\begin{aligned}
R f_{*} D_{X}\left(\Omega_{X}^{q}\right) \stackrel{\left({ }^{a} f^{*}\right)^{\vee}}{\longrightarrow} R f_{*} D_{X}\left(L f^{*} \Omega_{Y}^{q}\right) & \stackrel{c_{f, \pi_{Y}}}{\longrightarrow} R f_{*} R \mathcal{H} \operatorname{om}_{X}\left(L f^{*} \Omega_{Y}^{q}, f^{!} \pi_{Y}^{!} k\right) \\
& \stackrel{\text { adj. }}{\longrightarrow} R \mathcal{H} \operatorname{om}_{Y}\left(\Omega_{Y}^{q}, R f_{*} f^{!} \pi_{Y}^{!} k\right) \stackrel{\operatorname{Tr}_{f}}{\longrightarrow} D_{Y}\left(\Omega_{Y}^{q}\right) .
\end{aligned}
$$

Here adj. denotes the isomorphism

$$
R f_{*} R \operatorname{Hom}_{X}\left(L f^{*}(\cdot), \cdot\right) \cong R \operatorname{Hom}_{Y}\left(\cdot, R f_{*}(\cdot)\right) \quad \text { on } D_{\mathrm{c}}^{-}(Y) \times D^{+}(X)
$$

(see [Hartshorne 1966, Chapter II, Proposition 5.10]) and ${ }^{a} f^{*}: L f^{*} \Omega_{Y}^{q} \rightarrow \Omega_{X}^{q}$ is the morphism corresponding to $\Omega_{Y}^{q} \rightarrow R f_{*} \Omega_{X}^{q}$ under $H^{0}(Y, \cdot)$ applied to the isomorphism above.

Proposition 2.2.7. (1) $\mathrm{id}_{*}=\mathrm{id}$.

(2) Let $f: X \rightarrow Y$ and $g: Y \rightarrow Z$ be two proper maps with $X$ and $Y$ of pure dimension $d_{X}$ and $d_{Y}$, respectively. Then

$$
(g \circ f)_{*}=g_{*} \circ R g_{*}\left(f_{*}\right): R g_{*} R f_{*} D_{X}\left(\Omega_{X}^{q}\right) \rightarrow D_{Z}\left(\Omega_{Z}^{q}\right) .
$$

(3) Let

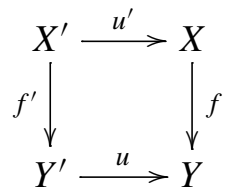

be a cartesian diagram with $f$ proper, $u$ étale and $X$ of pure dimension $d$. Then the diagram

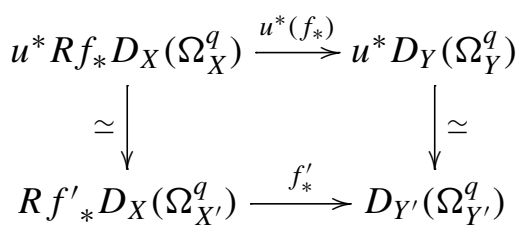

commutes, where the vertical maps are the natural isomorphisms (in the proof we will make these isomorphisms precise). 
Proof. (1) is clear. By [Conrad 2000, Lemma 3.4.3, (TRA1) and p. 139, (VAR1)], we have

$$
\operatorname{Tr}_{g \circ f}=\operatorname{Tr}_{g} \circ R g_{*}\left(\operatorname{Tr}_{f}\right) \circ R(g \circ f)_{*}\left(c_{f, g}\right): R g_{*} R f_{*}(g \circ f)^{!} \stackrel{\simeq}{\rightarrow} \mathrm{id} .
$$

and

$$
c_{f, g} \circ c_{g \circ f, h}=f^{!}\left(c_{g, h}\right) \circ c_{f, h \circ g}:(h \circ g \circ f)^{!} \rightarrow f^{!} g^{!} h^{!},
$$

where $h: Z \rightarrow W$ is a third map. This implies (2).

Now to make the vertical maps in (3) precise we need some further notation: Let

$$
\begin{aligned}
& \alpha: u^{*} R f_{*} \stackrel{\simeq}{\rightarrow} R f_{*} u^{\prime *}, \quad e_{u}: u^{*} \stackrel{\simeq}{\rightarrow} u^{!}, \\
& \beta_{u}: u^{*} R \mathscr{H o m}(\cdot, \cdot) \stackrel{\simeq}{\rightarrow} R \mathscr{H o m}\left(u^{*}(\cdot), u^{*}(\cdot)\right)
\end{aligned}
$$

be the natural isomorphisms. Then the vertical map on the left of (2.2.8) is given by $c_{u^{\prime}, \pi_{X}}^{-1} \circ e_{u^{\prime}} \circ \beta_{u^{\prime}} \circ \alpha$ and the vertical map on the right of (2.2.8) is given by $c_{u, \pi_{Y}}^{-1} \circ e_{u} \circ \beta_{u}$. Thus we have to prove

$$
c_{u, \pi_{Y}}^{-1} \circ e_{u} \circ \beta_{u} \circ u^{*}\left(f_{*}\right)=f_{*}^{\prime} \circ c_{u^{\prime}, \pi_{X}}^{-1} \circ e_{u^{\prime}} \circ \beta_{u^{\prime}} \circ \alpha .
$$

Denote by $b_{u, f}: u^{\prime *} f^{!} \stackrel{\simeq}{\rightarrow} f^{\prime !} u^{*}$ the isomorphism of [Hartshorne 1966, Chapter VII, Corollary 3.4(a)(5)]; see also [Conrad 2000, (3.3.24)]. Then it is easy (but tedious) to check that (2.2.11) follows from

$$
u^{*}\left(\operatorname{Tr}_{f}\right)=\operatorname{Tr}_{f^{\prime}} \circ R f^{\prime}{ }_{*}\left(b_{u, f}\right) \circ \alpha: u^{*} R f_{*} f^{!} \rightarrow R f^{\prime}{ }_{*} f^{\prime !} u^{*}
$$

(see [Conrad 2000, Lemma 3.4.3, (TRA4)]) and the following lemma.

Lemma 2.2.12. Let

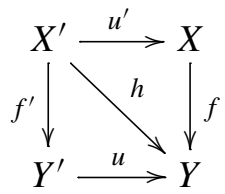

be a cartesian diagram with $u$ étale. Then the following diagram commutes (with notation as above):

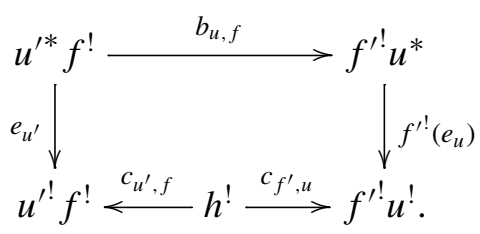

Proof. We extensively use the notation of [Hartshorne 1966; Conrad 2000]. All maps and functors involved in the statement are defined, for example, in [Conrad 2000, (3.3.6), (3.3.15), (3.3.21), (3.3.25)]. Using these definitions for the residual 
complex $K=\pi_{Y}^{\Delta} k$ on $Y$ together with the identity $u^{*} K=u^{\Delta} K$ and the commutativity of

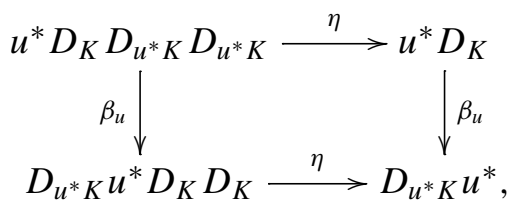

one checks that one is reduced to proving the commutativity of the diagram

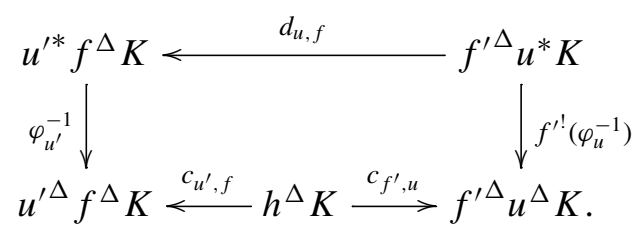

Here the maps are the analogs in the category of residual complexes of the maps in the lemma; see [Hartshorne 1966, Chapter IV, Theorems 3.1 and 5.5]. Since we work with actual complexes now, the commutativity of the diagram above is a local question. Thus take $U \subset X$ open such that $\left.f\right|_{U}$ factors as $U \stackrel{i}{\rightarrow} P \stackrel{p}{\rightarrow} Y$ with $i$ a closed immersion and $p$ smooth. Then $\left.f^{\prime}\right|_{U^{\prime}}$ also factors as $U^{\prime} \stackrel{i^{\prime}}{\rightarrow} P^{\prime} \stackrel{p^{\prime}}{\rightarrow} Y^{\prime}$. By the construction of $f^{\Delta}$ in the proof of [Hartshorne 1966, Chapter VI, Theorem 3.1(a)] we have $f^{\Delta}=i^{y} p^{z}$ and also $f^{\prime \Delta}=i^{\prime y} p^{\prime z}$. Now by [Hartshorne 1966, Chapter VI, Theorem 5.5(2)], we have

$$
c_{p, i} \circ d_{u, f}=d_{u_{P}, i} \circ i^{\prime \Delta}\left(d_{u, p}\right) \circ c_{p^{\prime}, i^{\prime}},
$$

with $u_{P}$ being the base change of $u$ by $p: P \rightarrow Y$. This equality implies that it sufficient to prove the commutativity of (2.2.13) for $i$ and $p$ separately. Thus we are reduced to consider the two cases $f$ is finite or $f$ is smooth. The latter case smooth is immediate, while the first follows from [Conrad 2000, Theorems 3.3.1, 2.(VAR4)].

Remark 2.2.14. (1) Let $\pi: X \rightarrow$ Spec $k$ be smooth of pure dimension $d$. Then there is a canonical isomorphism $\pi^{!} k \cong \Omega_{X}^{d}[d]=: \omega_{X}[d]$. More generally, for any $j \geq 0$ and $n \in \mathbb{Z}$ we have the isomorphism

$$
\Omega_{X}^{j}[n] \stackrel{\simeq}{\rightarrow} D_{X}\left(\Omega_{X}^{d-j}\right)[n-d],
$$

which is defined to be the composition of

$$
\Omega_{X}^{j}[n] \stackrel{\simeq}{\longrightarrow} \operatorname{Hom}_{X}\left(\Omega_{X}^{d-j}, \Omega_{X}^{d}\right)[n], \quad \alpha \mapsto(\beta \mapsto \alpha \wedge \beta)
$$

(notice that we make the choice of a sign here) with $\operatorname{Hom}_{X}\left(\Omega_{X}^{d-j}, \Omega_{X}^{d}\right)[n]=\mathscr{H}_{\mathrm{om}}^{\bullet}\left(\Omega_{X}^{d-j}, \Omega_{X}^{d}[d]\right)[n-d] \cong R \mathscr{H o m}\left(\Omega_{X}^{d-j}, \pi^{!} k\right)[n-d]$. 
(2) Let $X$ be a $k$-scheme of pure dimension $d$ and $U \subset X$ a smooth open subscheme; then we have for all $j \geq 0$

$$
\left.\Omega_{U}^{j} \simeq \operatorname{Hom}_{U}^{\bullet}\left(\Omega_{U}^{d-j}, \Omega_{U}^{d}[d]\right)[-d] \simeq D_{X}\left(\Omega_{X}^{d-j}\right)\right|_{U}[-d],
$$

where the first isomorphism is as in (1) and the second is given by restriction (or to be more precise, first use the isomorphism $\Omega_{U}^{d}[d] \simeq \pi_{U}^{!} k$ and then the vertical isomorphism on the right in (2.2.8) with $U \hookrightarrow X$ instead of $u: Y^{\prime} \rightarrow Y$ ).

Lemma 2.2.16. Let $\pi_{X}: X \rightarrow$ Spec $k$ be proper of pure dimension $d_{X}$ and let $\pi_{Y}: Y \rightarrow$ Spec $k$ be smooth of pure dimension $d_{Y}$. We denote by $\mathrm{pr}_{2}: X \times Y \rightarrow Y$ the projection (it is proper) and set $d:=\operatorname{dim}(X \times Y)$. Then for all $j \geq 0$, there is a morphism in $D_{\mathrm{c}}^{+}(X \times Y)$

$$
\gamma: \operatorname{pr}_{2}^{!}\left(\mathscr{O}_{Y}\right) \otimes\left(\operatorname{pr}_{2}^{*} \Omega_{Y}^{j-d_{X}}\left[d_{Y}\right]\right) \rightarrow D_{X \times Y}\left(\operatorname{pr}_{2}^{*} \Omega_{Y}^{d-j}\right)
$$

satisfying the following conditions:

(1) For $U \subset X$ open and smooth denote by $p_{2}: U \times X \rightarrow Y$ the restriction of $\mathrm{pr}_{2}$. Then $\left.\gamma\right|_{U \times Y}$ is the composition $\left.\left(\operatorname{pr}_{2}^{!}\left(\mathscr{O}_{Y}\right) \otimes \operatorname{pr}_{2}^{*} \Omega_{Y}^{j-d_{X}}\left[d_{Y}\right]\right)\right|_{U \times Y} \stackrel{\simeq}{\rightarrow} \Omega_{U \times Y / Y}^{d_{X}}\left[d_{X}\right] \otimes p_{2}^{*} \Omega_{Y}^{j-d_{X}}\left[d_{Y}\right]$

$$
\stackrel{\simeq}{\longrightarrow} \Omega_{U \times Y / Y}^{d_{X}}\left[d_{X}\right] \otimes p_{2}^{*} R \mathcal{H o m}_{Y}\left(\Omega_{Y}^{d-j}, \Omega_{Y}^{d_{Y}}\left[d_{Y}\right]\right) \stackrel{\simeq \text { nat. }}{\longrightarrow} D_{U \times Y}\left(p_{2}^{*} \Omega_{Y}^{d-j}\right) .
$$

Here the last isomorphism is induced by the composition of the canonical isomorphisms

$$
\Omega_{U \times Y / Y}^{d_{X}}\left[d_{X}\right] \otimes p_{2}^{*} \Omega_{Y}^{d_{Y}}\left[d_{Y}\right] \cong \Omega_{U \times Y}^{d}[d] \cong \pi_{U \times Y}^{!}(k) .
$$

(2) The following diagram commutes:

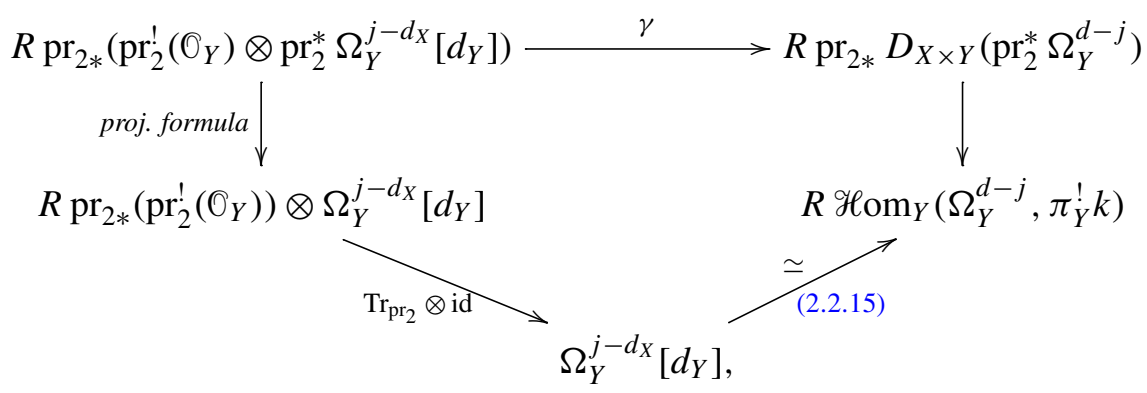

where the vertical map on the right is $\operatorname{Tr}_{\mathrm{pr}_{2}} \circ$ adjunction $\circ c_{\mathrm{pr}_{2}, \pi_{Y}}$.

Proof. Conrad [2000, (4.3.12)] defines a map

$$
e_{\mathrm{pr}_{2}}: \mathrm{pr}_{2}^{!}\left(\mathrm{O}_{Y}\right) \otimes \operatorname{pr}_{2}^{*} \pi_{Y}^{!} k \longrightarrow \mathrm{pr}_{2}^{!} \pi_{Y}^{!} k
$$


such that

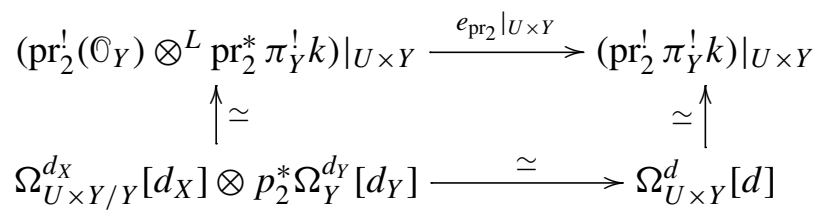

commutes, where the vertical map on the left is the composition of the canonical isomorphism $\Omega_{U \times Y}^{d}[d] \cong \pi_{U \times Y}^{!}(k)$ with $c_{p_{2}, \pi_{Y}}: \pi_{U \times Y}^{!} \cong p_{2}^{!} \pi_{Y}^{!}$. Furthermore by [Conrad 2000, Theorem 4.4.1] the following diagram commutes:

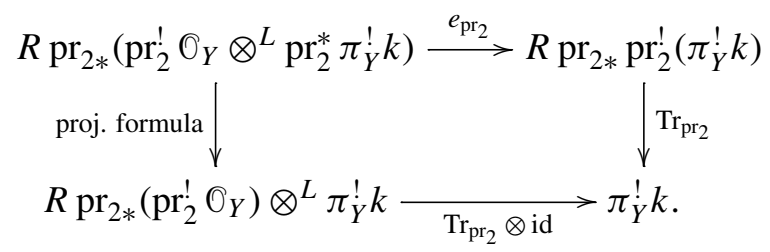

We define $\gamma$ to be the composition

$$
\begin{aligned}
& \operatorname{pr}_{2}^{!}\left(O_{Y}\right) \otimes \operatorname{pr}_{2}^{*} \Omega_{Y}^{j-d_{X}}\left[d_{Y}\right] \stackrel{\operatorname{id} \otimes(2.2 .15)}{\longrightarrow} \operatorname{pr}_{2}^{!}\left(O_{Y}\right) \otimes \operatorname{pr}_{2}^{*} R \mathscr{H o m}{ }_{Y}\left(\Omega_{Y}^{d-j}, \pi_{Y}^{!} k\right) \\
& \stackrel{\text { nat. }}{\longrightarrow} R \mathscr{H o m}\left(\mathrm{pr}_{2}^{*} \Omega_{Y}^{d-j}, \mathrm{pr}_{2}^{!}\left(\mathrm{O}_{Y}\right) \otimes^{L} \mathrm{pr}_{2}^{*} \pi_{Y}^{!} k\right) \\
& \stackrel{c_{\mathrm{pr}_{2}, \pi_{Y}}^{-1} \circ e_{\mathrm{pr}_{2}}^{\longrightarrow}}{\longrightarrow} D_{X \times Y}\left(\mathrm{pr}_{2}^{*} \Omega_{Y}^{d-j}\right) .
\end{aligned}
$$

It follows from (2.2.17) and (2.2.18) that $\gamma$ satisfies (1) and (2).

Proposition 2.2.19. Let $i: X \hookrightarrow Y$ be a closed immersion of pure codimension c between smooth $k$-schemes of pure dimension $d_{X}$ and $d_{Y}$, respectively. Then

$$
R \Gamma_{X} \Omega_{Y}^{q}[c] \cong \mathscr{H}_{X}^{c}\left(\Omega_{Y}^{q}\right) \text { in } D_{\mathrm{qc}}^{b}\left(\mathcal{O}_{Y}\right) \text { for all } q \geq 0
$$

Suppose further the ideal sheaf of $X$ in $0_{Y}$ is generated by a sequence $t=t_{1}, \ldots, t_{c}$ of global sections of $\mathrm{O}_{Y}$. Define a morphism $\imath_{X}^{q}$ by

$$
\imath_{X}^{q}: i_{*} \Omega_{X}^{q} \rightarrow \mathscr{H}_{X}^{c}\left(\Omega_{Y}^{c+q}\right), \quad \alpha \mapsto(-1)^{c}\left[\begin{array}{c}
d t \tilde{\alpha} \\
t
\end{array}\right]
$$


where $\tilde{\alpha} \in \Omega_{Y}^{q}$ is any lift of $\alpha$ and $d t=d t_{1} \wedge \cdots \wedge d t_{c}$. (Here we use the notation of Section A.1.) Then the following diagram commutes in $D_{\mathrm{qc}}^{b}\left(\mathrm{O}_{Y}\right)$ :

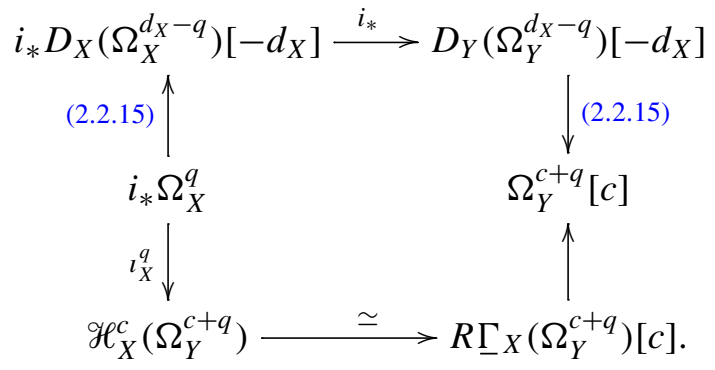

Proof. The first statement is well known; see also Lemma A.2.5. It remains to prove the commutativity of (2.2.20). Let $\pi_{X}: X \rightarrow \operatorname{Spec} k$ and $\pi_{Y}: Y \rightarrow$ Spec $k$ be the structure maps. By Definition 2.2.5, the top row in (2.2.20) is given by the following composition in $D_{\mathrm{qc}}^{b}\left(\mathrm{O}_{Y}\right)$ :

$$
\begin{aligned}
i_{*} \Omega_{X}^{q} & \stackrel{(2.2 .15)}{\longrightarrow} i_{*} R \mathscr{H o m}\left(\Omega_{X}^{d_{X}-q}, \pi_{X}^{!} k\right)\left[-d_{X}\right] \\
& \stackrel{c_{i, \pi_{Y}}}{\longrightarrow} i_{*} R \mathscr{H o m}\left(\Omega_{X}^{d_{X}-q}, i^{!} \pi_{Y}^{!} k\right)\left[-d_{X}\right] \\
& \stackrel{\text { nat. }}{\longrightarrow} R \mathscr{H o m}\left(i_{*} \Omega_{X}^{d_{X}-q}, i_{*} i^{!} \pi_{Y}^{!} k\right)\left[-d_{X}\right] \\
& \stackrel{\mathrm{Tr}_{i}}{\longrightarrow} R \mathscr{H o m}\left(i_{*} \Omega_{X}^{d_{X}-q}, \pi_{Y}^{!} k\right)\left[-d_{X}\right] \\
& \stackrel{\left(i^{*}\right)^{\vee}}{\longrightarrow} R \mathscr{H o m}\left(\Omega_{Y}^{d_{X}-q}, \pi_{Y}^{!} k\right)\left[-d_{X}\right] \stackrel{(2.2 .15)^{-1}}{\longrightarrow} \Omega_{Y}^{c+q}[c] .
\end{aligned}
$$

We set $\iota_{X}:=l_{X}^{d_{X}}$. Then it follows from Lemma A.2.12 and the definition of (2.2.15) that the composition above equals

$$
\begin{aligned}
i_{*} \Omega_{X}^{q} & \stackrel{\text { multipl. }}{\longrightarrow} i_{*} \operatorname{Hoom}\left(\Omega_{X}^{d_{X}-q}, \Omega_{X}^{d_{X}}\right) \\
& \stackrel{\text { nat. }}{\longrightarrow} \mathscr{H o m}\left(i_{*} \Omega_{X}^{d_{X}-q}, i_{*} \Omega_{X}^{d_{X}}\right) \\
& \stackrel{l_{X}}{\longrightarrow} \mathscr{H o m}\left(i_{*} \Omega_{X}^{d_{X}-q}, \mathscr{H}_{X}^{c}\left(\Omega_{Y}^{d_{Y}}\right)\right) \\
& \stackrel{\left(i^{*}\right)^{\vee}}{\longrightarrow} \mathscr{H o m}\left(\Omega_{Y}^{d_{X}-q}, \mathscr{H}_{X}^{c}\left(\Omega_{Y}^{d_{Y}}\right)\right) \\
& \stackrel{(*)}{\longrightarrow} \operatorname{Hom}^{\bullet}\left(\Omega_{Y}^{d_{X}-q}, \Omega_{Y}^{d_{Y}}[c]\right) \stackrel{\text { multipl. }{ }^{-1}}{\longrightarrow} \Omega_{Y}^{c+q}[c],
\end{aligned}
$$

where $(*)$ is induced by $\mathscr{H}_{X}^{c}\left(\Omega_{Y}^{d_{Y}}\right) \cong R \Gamma_{X}\left(\Omega_{Y}^{d_{Y}}\right)[c] \rightarrow \Omega_{Y}^{d_{Y}}[c]$. There is a natural isomorphism

$$
\varphi: \mathscr{H}_{X}^{c}\left(\Omega_{Y}^{c+q}\right) \stackrel{\simeq}{\rightarrow} \operatorname{Hom}\left(\Omega_{Y}^{d_{X}-q}, \mathscr{H}_{X}^{c}\left(\Omega_{Y}^{d_{Y}}\right)\right)
$$


coming from the isomorphisms

$$
\begin{aligned}
\mathscr{H o m}\left(\Omega_{Y}^{d_{X}-q}, \mathscr{H}_{X}^{c}\left(\Omega_{Y}^{d_{Y}}\right)\right) & \cong R \mathscr{H o m}\left(\Omega_{Y}^{d_{X}-q}, R \underline{\Gamma}_{X}\left(\Omega_{Y}^{d_{Y}}\right)[c]\right) \\
& \cong R \underline{\Gamma}_{X}\left(R \mathscr{H} \operatorname{om}\left(\Omega_{Y}^{d_{X}-q}, \Omega_{Y}^{d_{Y}}\right)\right)[c] \cong R \underline{\Gamma}_{X}\left(\Omega_{Y}^{c+q}\right)[c] \\
& \cong \mathscr{H}_{X}^{c}\left(\Omega_{Y}^{c+q}\right) .
\end{aligned}
$$

This isomorphism is explicitly given by

$$
\varphi: \mathscr{H}_{X}^{c}\left(\Omega_{Y}^{c+q}\right) \stackrel{\simeq}{\longrightarrow} \mathscr{H o m}\left(\Omega_{Y}^{d_{X}-q}, \mathscr{H}_{X}^{c}\left(\Omega_{Y}^{d_{Y}}\right)\right), \quad\left[\begin{array}{c}
\alpha \\
t^{n}
\end{array}\right] \mapsto\left(\beta \mapsto\left[\begin{array}{c}
\alpha \beta \\
t^{n}
\end{array}\right]\right) .
$$

The composition (2.2.21) equals

$$
i_{*} \Omega_{X}^{q} \stackrel{\varphi^{-1} \circ\left(i^{*}\right)^{\vee} \circ l_{X} \circ \text { (nat.)o(multipl.) }}{\longrightarrow} \mathcal{H}_{X}^{c}\left(\Omega_{Y}^{c+q}\right) \cong R \underline{\Gamma}{ }_{X}\left(\Omega_{Y}^{c+q}\right)[c] \rightarrow \Omega_{Y}^{c+q}[c] .
$$

It is straightforward to check that $\imath_{X}^{q}=\varphi^{-1} \circ\left(i^{*}\right)^{\vee} \circ l_{X} \circ$ (nat.) $\circ$ (multipl.), and this implies the commutativity of (2.2.20).

Corollary 2.2.22. Assume we have a cartesian square

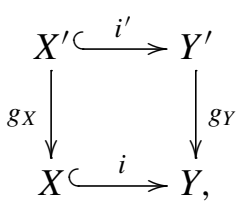

in which $X, X^{\prime}, Y, Y^{\prime}$ are smooth of pure dimension $d_{X}, d_{X^{\prime}}, d_{Y}, d_{Y^{\prime}}$, i is a closed immersion, and $c:=d_{Y}-d_{X}=d_{Y^{\prime}}-d_{X^{\prime}}$. Then for all $q \geq 0$ the following diagram commutes in $D_{\mathrm{qc}}^{b}(Y)$ :

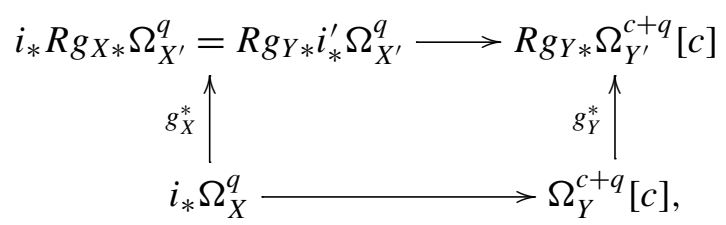

where the lower horizontal morphism is given by the composition

$$
i_{*} \Omega_{X}^{q} \stackrel{(2.2 .15)}{\longrightarrow} i_{*} D_{X}\left(\Omega_{X}^{d_{X}-q}\right)\left[-d_{X}\right] \stackrel{i_{*}}{\rightarrow} D_{Y}\left(\Omega_{Y}^{d_{X}-q}\right)\left[-d_{X}\right] \stackrel{(2.2 .15)}{\longrightarrow} \Omega_{Y}^{c+q}[c]
$$

and the upper horizontal morphism by $\operatorname{Rg}_{Y *}$ applied to the analogous map for $i^{\prime}$. Proof. Since $R \underline{\Gamma}_{X} R g_{Y *}=R g_{Y *} R \underline{\Gamma}_{X^{\prime}}$, we naturally have a commutative diagram

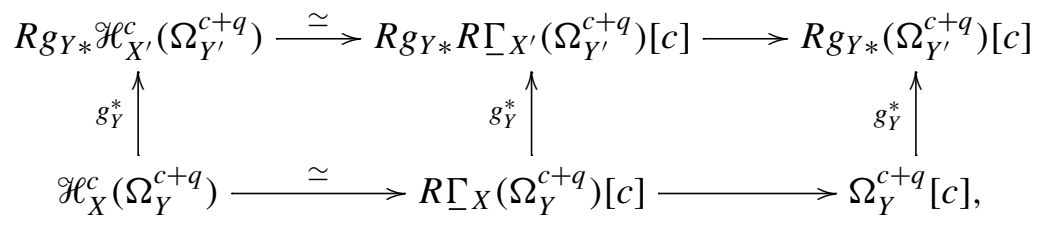


where the $g_{Y}^{*}$ on the very left is defined in such a way that the left square commutes. By Proposition 2.2.19 it thus suffices to prove the commutativity of

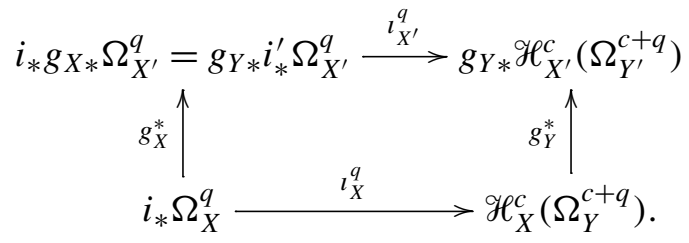

This is a local question, we may therefore assume that the ideal of $X$ in $Y$ is generated by a sequence $t_{1}, \ldots, t_{c}$ of global sections of $\mathscr{O}_{Y}$. Then $g_{Y}^{*} t_{1}, \ldots, g_{Y}^{*} t_{c}$ is a sequence of global sections of $\mathrm{O}_{Y^{\prime}}$, which generate the ideal sheaf of $X^{\prime}$ in $Y^{\prime}$. Hence the assumption follows from the explicit description of $\imath_{X}^{q}$ and $\imath_{X^{\prime}}^{q}$ in Proposition 2.2.19.

Proposition 2.2.23. Let $f: X \rightarrow Y$ be a finite and surjective morphism between smooth schemes, which are both of pure dimension $n$. We denote by

$$
\tau_{f}: \bigoplus_{q} f_{*} \Omega_{X}^{q} \rightarrow \bigoplus_{q} \Omega_{Y}^{q}
$$

the composition

$$
\bigoplus_{q} f_{*} \Omega_{X}^{q} \stackrel{(2.2 .15)}{\longrightarrow} \bigoplus_{q} f_{*} D_{X}\left(\Omega_{X}^{n-q}\right) \stackrel{f_{*}}{\rightarrow} \bigoplus_{q} D_{Y}\left(\Omega_{Y}^{n-q}\right) \stackrel{(2.2 .15)}{\longrightarrow} \bigoplus_{q} \Omega_{Y}^{q} .
$$

Then we have the following:

(1) In degree 0, the map $\tau_{f}$ equals the usual trace on the finite and locally free $\mathrm{O}_{Y}$-module $f_{*} \mathrm{O}_{X}$, that is, $\operatorname{Tr}_{X / Y}: f_{*} \mathrm{O}_{X} \rightarrow \mathrm{O}_{Y}$.

(2) For $\alpha \in f_{*} \Omega_{X}^{a}$ and $\beta \in \Omega_{Y}^{b}$, we have

$$
\tau_{f}\left(\alpha f^{*} \beta\right)=\tau_{f}(\alpha) \beta .
$$

(3) The composition $\tau_{f} \circ f^{*}: \bigoplus_{q} \Omega_{Y}^{q} \rightarrow \bigoplus_{q} \Omega_{Y}^{q}$ equals multiplication with the degree of $f$.

Proof. All statements are local in $Y$. We may therefore assume that $f$ factors as $X \stackrel{i}{\rightarrow} P \stackrel{\pi}{\rightarrow} Y$, where $i$ is a regular closed immersion of pure codimension $d$ and $\pi$ is smooth of relative dimension $d$; further we may assume that the ideal sheaf of $X$ in $P$ is generated by $d$ global sections $t_{1}, \ldots, t_{d}$ of $O_{P}$. Then in degree $n$ the map $\tau_{f}$ equals the trace map $\tau_{f}^{n}: f_{*} \omega_{X} \rightarrow \omega_{Y}$ from Section 1.3.1 and in degree $q$ the map $\tau_{f}$ thus equals the composition

$$
\begin{aligned}
f_{*} \Omega_{X}^{q} \cong f_{*} \operatorname{Hom}_{X}\left(\Omega_{X}^{n-q}, \omega_{X}\right) & \stackrel{\text { nat. }}{\longrightarrow} \mathcal{H}_{\mathrm{om}_{Y}}\left(f_{*} \Omega_{X}^{n-q}, f_{*} \omega_{X}\right) \\
& \stackrel{\tau_{f}^{n}}{\rightarrow} \mathcal{H o m}\left(f_{*} \Omega_{X}^{n-q}, \omega_{Y}\right) \stackrel{\circ f^{*}}{\longrightarrow} \operatorname{Hom}\left(\Omega_{Y}^{n-q}, \omega_{Y}\right) \cong \Omega_{Y}^{q} .
\end{aligned}
$$


Thus for $\alpha \in f_{*} \Omega_{X}^{q}$, Lemma A.3.3 gives the following formula for $\tau_{f}(\alpha)$ : In

$$
i^{*} \Omega_{P}^{d+q}=\bigoplus_{r+s=d+q} i^{*}\left(\Omega_{P / Y}^{r}\right) \otimes f^{*} \Omega_{Y}^{s},
$$

write

$i^{*}\left(d t_{d} \wedge \cdots \wedge d t_{1} \wedge \tilde{\alpha}\right)=\sum_{r+s=d+q} \sum_{j} i^{*} \gamma_{j, r} \otimes f^{*} \beta_{j, s} \quad$ for $\gamma_{j, r} \in \Omega_{P / Y}^{r}, \beta_{j, s} \in \Omega_{Y}^{s}$,

where $\tilde{\alpha} \in \Omega_{P}^{q}$ is a lift of $\alpha$. Then

$$
\tau_{f}(\alpha)=(-1)^{d(d-1) / 2} \sum_{j} \operatorname{Res}_{P / Y}\left[\begin{array}{c}
\gamma_{j, d} \\
t_{1}, \ldots, t_{d}
\end{array}\right] \beta_{j, q} \in \Omega_{Y}^{q} .
$$

This formula immediately implies (2). For any $a \in \mathrm{O}_{X}$, we have

$$
\tau_{f}(a)=(-1)^{d(d-1) / 2} \operatorname{Res}_{P / Y}\left[\begin{array}{c}
\tilde{a} d t_{d} \wedge \cdots \wedge d t_{1} \\
t_{1}, \ldots, t_{d}
\end{array}\right]=\operatorname{Res}_{P / Y}\left[\begin{array}{c}
\tilde{a} d t_{1} \wedge \cdots \wedge d t_{d} \\
t_{1}, \ldots, t_{d}
\end{array}\right],
$$

which equals $\operatorname{Tr}_{X / Y}(a)$ by [Conrad 2000, page 240, (R6)]; hence (1). Finally (3) is a direct consequence of (1) and (2).

Remark 2.2.25. The trace map from Proposition 2.2 .23 and its properties are well known; see for example [Kunz 1986, §16], where the trace is considered in much greater generality. There the construction is done via an ad hoc method not using the duality formalism. Therefore the connection to the trace map above is not a priori clear.

\subsection{Push-forward for Hodge cohomology with support.}

Definition 2.3.1. Let $f:(X, \Phi) \rightarrow(Y, \Psi)$ be a morphism in $V_{*}$ with $X$ equidimensional. We define a compactification of $f$ to be a factorization

$$
f=\bar{f} \circ j:(X, \Phi) \hookrightarrow(\bar{X}, \Phi) \rightarrow(Y, \Psi),
$$

where $\bar{X}$ is equidimensional (but possibly singular), $j$ is an open immersion and $\bar{f}$ is proper. Notice that since $\left.f\right|_{\Phi}$ is proper, $\Phi$ is also a family of supports on $\bar{X}$. The compactification will be denoted by $(j, \bar{f})$.

By Nagata's compactification theorem (see, for example [Conrad 2007]) any $f$ in $V_{*}$ admits a compactification.

Definition 2.3.2 (push-forward). Let $f:(X, \Phi) \rightarrow(Y, \Psi)$ be a morphism in $V_{*}$ and assume that $X$ and $Y$ are of pure dimension $d_{X}$ and $d_{Y}$, respectively, and set $r:=d_{X}-d_{Y}$. Let

$$
(X, \Phi) \stackrel{j}{\rightarrow}(\bar{X}, \Phi) \stackrel{\bar{f}}{\rightarrow}(Y, \Psi)
$$


be a compactification of $f$. We define the push-forward

$$
H_{*}(f): H(X, \Phi) \rightarrow H(Y, \Psi)
$$

as the composition

$$
\begin{aligned}
H(X, \Phi) & \simeq \bigoplus_{i, j} H_{\Phi}^{i}\left(\bar{X}, D_{\bar{X}}\left(\Omega_{\bar{X}}^{d_{X}-j}\right)\left[-d_{X}\right]\right) \stackrel{\text { nat. }}{\longrightarrow} \bigoplus_{i, j} H_{\bar{f}^{-1}(\Psi)}^{i-d_{X}}\left(\bar{X}, D_{\bar{X}}\left(\Omega_{\bar{X}}^{d_{X}-j}\right)\right) \\
& \stackrel{\oplus \bar{f}_{*}^{j}}{\longrightarrow} \bigoplus_{i, j} H_{\Psi}^{i-d_{X}}\left(Y, D_{Y}\left(\Omega_{Y}^{d_{X}-j}\right)\right) \stackrel{\simeq(2.2 .15)}{\longrightarrow} \bigoplus_{i, j} H_{\Psi}^{i-r}\left(Y, \Omega_{Y}^{j-r}\right)=H(Y, \Psi),
\end{aligned}
$$

where the first isomorphism is the composition of (2.2.15) for $n=0$ with the excision isomorphism. Notice that we obtain a morphism of graded abelian groups $H_{*}(f): H_{*}(X, \Phi) \rightarrow H_{*}(Y, \Psi)$; see (2.0.2).

This definition is independent of the chosen compactification.

We extend the definition to the case of nonequidimensional $X$ and $Y$ additively. Proof. We have to prove the independence of $H_{*}(f)$ from the chosen compactification. Let

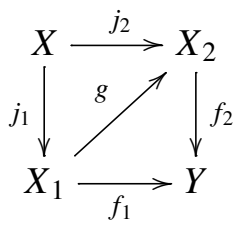

be a commutative diagram with $d:=\operatorname{dim} X_{1}=\operatorname{dim} X_{2}=d_{X}, j_{1}$ and $j_{2}$ open and $f_{1}$ and $f_{2}$ proper. Notice that $g$ is automatically proper. Then the diagram

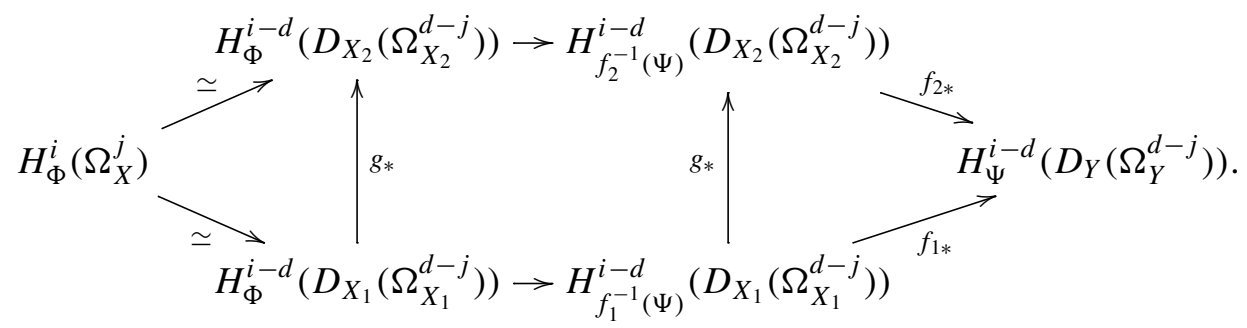

commutes. The left triangle commutes since $\left.g_{*}\right|_{X}=\mathrm{id}_{*}$ by Proposition 2.2.7(3), the square in the middle obviously commutes, and the triangle on the right commutes by Proposition 2.2.7(2).

Two arbitrary compactifications of $f$ always receive a map from a third one and thus the general case follows from the case above.

Proposition 2.3.3. (1) $H_{*}($ id $)=$ id.

(2) Let $f:(X, \Phi) \rightarrow(Y, \Psi)$ and $g:(Y, \Psi) \rightarrow(Z, \Xi)$ be two morphisms in $V_{*}$. Then $H_{*}(g \circ f)=H_{*}(g) \circ H_{*}(f): H(X, \Phi) \rightarrow H(Z, \Xi)$. 
(3) If $f:(X, \Phi) \rightarrow(Y, \Psi)$ in $V_{*}$ is finite, then $H_{*}(f)$ is induced by the trace map $\tau_{f}$ from Proposition 2.2.23.

Proof. (1) follows from Proposition 2.2.7(1). Now for (2) we may assume that $X, Y$ and $Z$ are connected. Let $\left(j_{X}, f_{1}\right)$ and $\left(j_{Y}, g_{1}\right)$ be compactifications of $f$ and $g$, respectively. Let $\left(j_{X_{1}}, f_{2}\right)$ be a compactification of $j_{Y} \circ f_{1}$. Thus we have a commutative diagram

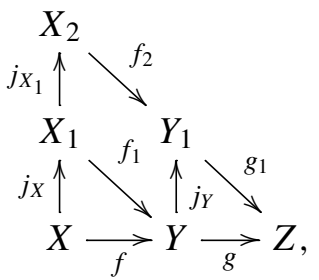

with vertical arrows open immersions and diagonal arrows proper. By replacing $X_{1}$ by $f_{2}^{-1}(Y)$, we may assume that the parallelogram is cartesian. Then by Proposition 2.2.7(3) the diagram

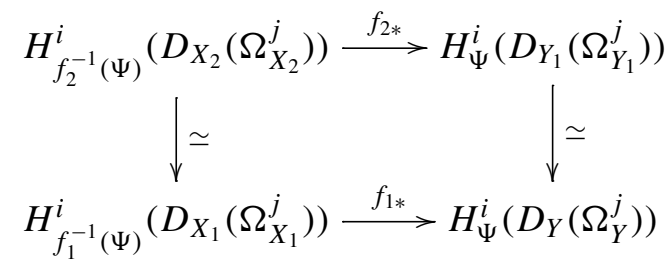

commutes. Thus (2) follows from Proposition 2.2.7(2). Finally (3) follows immediately from the definitions.

Lemma 2.3.4. Consider a cartesian diagram

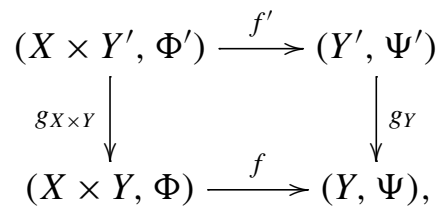

such that $f$ is induced by the projection to $Y$, with $f, f^{\prime} \in V_{*}$ and $g_{X \times Y}, g_{Y} \in V^{*}$. Then $H^{*}\left(g_{Y}\right) \circ H_{*}(f)=H_{*}\left(f^{\prime}\right) \circ H^{*}\left(g_{X \times Y}\right)$.

Furthermore, $H_{*}(f): H(X \times Y, \Phi) \rightarrow H(Y, \Psi)$ factors over the projection

$$
H(X \times Y, \Phi) \rightarrow \bigoplus_{i, j} H_{\Phi}^{i}\left(X \times Y, \operatorname{pr}_{1}^{*} \Omega_{X}^{d_{X}} \otimes \operatorname{pr}_{2}^{*} \Omega_{Y}^{j}\right) .
$$

Proof. We may assume $X$ and $Y$ are of pure dimension $d_{X}$ and $d_{Y}$, respectively, and we set $d:=d_{X}+d_{Y}$. We embed $X$ as an open in a proper $k$-scheme $\bar{X}$ of pure dimension $d_{X}$. Then

$$
(X \times Y, \Phi) \stackrel{j}{\rightarrow}(\bar{X} \times Y, \Phi) \stackrel{\mathrm{pr}_{2}}{\longrightarrow}(Y, \Psi)
$$


is a compactification of $f$, where $j$ is the open embedding and $\mathrm{pr}_{2}$ is induced by the projection to $Y$. Similarly we obtain a compactification for $f^{\prime}$, in which case we write $\mathrm{pr}_{2}^{\prime}$ for the projection to $Y^{\prime}$. The second statement of the lemma follows from Definition 2.3.2, Remark 2.2.6(2) and the commutative diagram

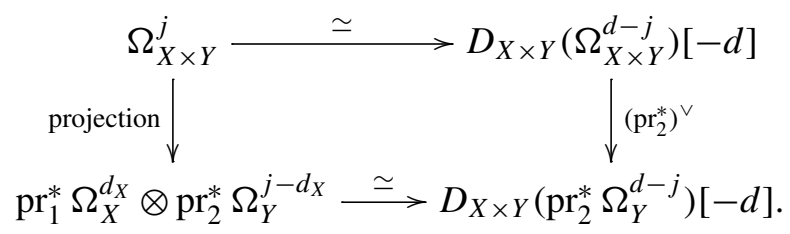

Now we come to the first statement of the lemma. Consider the diagram (we use a shortened notation)

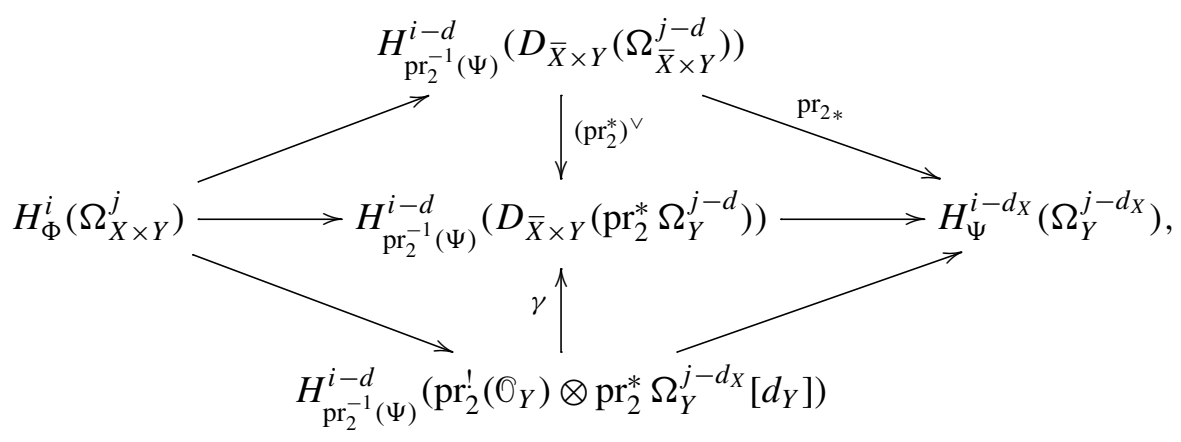

where we use the notation of Lemma 2.2.16, the upper map on the left is induced by excision, the middle and the lower map on the left are induced by projection and excision, and the middle and the lower map on the right are induced by the corresponding maps from Lemma 2.2.16(2). It follows from Lemma 2.2.16 and Remark 2.2.6(2) that all the triangles in this diagram commute. Replacing $Y$ by $Y^{\prime}$ and $\mathrm{pr}_{2}$ by $\mathrm{pr}_{2}^{\prime}$, we obtain a similar commutative diagram. Thus it remains to show that the diagram

$$
\begin{aligned}
& H_{\Phi}^{i}\left(\Omega_{X \times Y}^{j}\right) \stackrel{\text { proj. }}{\longrightarrow} H_{\mathrm{pr}_{2}^{-1}(\Psi)}^{i-d}\left(\operatorname{pr}_{2}^{!}\left(\mathrm{O}_{Y}\right) \otimes \mathrm{pr}_{2}^{*} \Omega_{Y}^{j-d_{X}}\left[d_{Y}\right]\right) \stackrel{\mathrm{Tr}_{\mathrm{pr}_{2}} \otimes \mathrm{id}}{\longrightarrow} H_{\Psi}^{i-d_{X}}\left(\Omega_{Y}^{j-d_{X}}\right) \\
& \quad H^{*}\left(g_{X \times Y}\right) \\
& H_{\Phi^{\prime}}^{i}\left(\Omega_{X \times Y^{\prime}}^{j}\right) \stackrel{\text { proj. }}{\longrightarrow} H_{\mathrm{pr}_{2}^{\prime-1}\left(\Psi^{\prime}\right)}^{i-d^{\prime}}\left(\operatorname{pr}_{2}^{\prime} !\left(O_{Y^{\prime}}\right) \otimes \operatorname{pr}_{2}^{\prime *} \Omega_{Y^{\prime}}^{j-d_{X}}\left[d_{Y^{\prime}}\right]\right) \stackrel{\operatorname{Tr}_{\mathrm{pr}_{2}^{\prime}} \otimes \mathrm{id}}{\longrightarrow} H_{\Psi^{\prime}}^{i-d_{X}}\left(\Omega_{Y^{\prime}}^{j-d_{X}}\right)
\end{aligned}
$$

is commutative, where $d^{\prime}=d_{X}+d_{Y^{\prime}}$. To this end we define the map

$$
\tau_{f}: R f_{*} R \underline{\Gamma}_{\Phi}\left(\omega_{X \times Y / Y}\left[d_{X}\right]\right) \rightarrow R \underline{\Gamma}_{\Psi} \widehat{O}_{Y}
$$


to be the composition

$$
\begin{aligned}
& R f_{*} R \Gamma_{\Phi}\left(\omega_{X \times Y / Y}\left[d_{X}\right]\right) \stackrel{\text { excision } \simeq}{\longrightarrow} R \operatorname{pr}_{2 *} R \underline{\Gamma_{\Phi}}\left(\operatorname{pr}_{2}^{!} O_{Y}\right)
\end{aligned}
$$

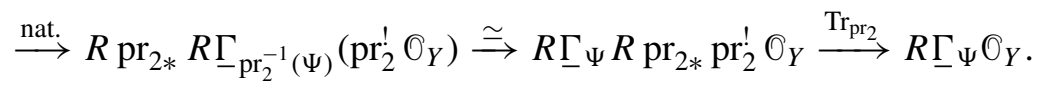

Then the upper horizontal line in diagram (2.3.5) equals $H^{i-d}(Y, \cdot)$ applied to the composition

$$
\begin{aligned}
R f_{*} R \underline{\Gamma}_{\Phi} \Omega_{X \times Y}^{j}[d] & \stackrel{\text { projection }}{\longrightarrow} R f_{*} R \underline{\Gamma}_{\Phi}\left(\omega_{X \times Y / Y}\left[d_{X}\right] \otimes f^{*} \Omega_{Y}^{j-d_{X}}\left[d_{Y}\right]\right) \\
& \simeq R f_{*} R \underline{\Gamma}_{\Phi}\left(\omega_{X \times Y / Y}\left[d_{X}\right]\right) \otimes \Omega_{Y}^{j-d_{X}}\left[d_{Y}\right] \stackrel{\tau_{f} \otimes \mathrm{id}}{\longrightarrow} R \underline{\Gamma}_{\Psi}\left(\Omega_{Y}^{j-d_{X}}\right)\left[d_{Y}\right] .
\end{aligned}
$$

(That there is no intervention of signs in the definition of the projection map is compatible with the fact that the isomorphism $\omega_{X \times Y}[d] \cong \omega_{X \times Y / Y}\left[d_{X}\right] \otimes f^{*} \omega_{Y}\left[d_{Y}\right]$ is defined without a sign; see [Conrad 2000, (2.2.6)].) The lower horizontal line in the diagram (2.3.5) equals $H^{i-d^{\prime}}\left(Y^{\prime}, \cdot\right)$ applied to the analog composition for $f^{\prime}$. Then it is straightforward to check that the commutativity of diagram (2.3.5) is implied by the commutativity of

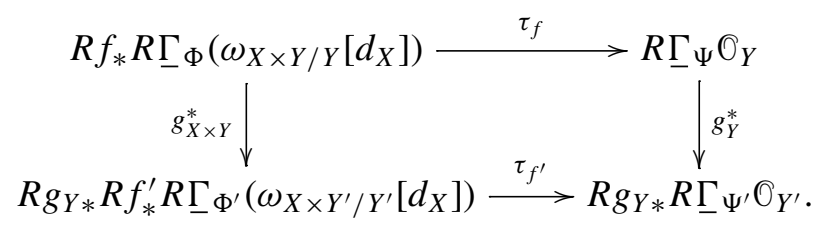

To prove the commutativity of this last diagram, we can clearly assume (by definition of the pull-back and $\tau_{f}$ ) that $\Phi^{\prime}=g_{X \times Y}^{-1}(\Phi)$ and $\Psi^{\prime}=g_{Y}^{-1}(\Psi)$. We define the map

$$
\alpha: R \operatorname{pr}_{2 *} \operatorname{pr}_{2}^{!} \mathrm{O}_{Y} \rightarrow R g_{Y *} R \operatorname{pr}_{2 *}^{\prime}\left(\mathrm{pr}_{2}^{\prime}\right)^{!} \mathrm{O}_{Y^{\prime}}
$$

to be the composition

$$
\begin{aligned}
R \operatorname{pr}_{2 *} \operatorname{pr}_{2}^{!}\left(\pi_{Y}^{*} k\right) & \stackrel{b_{\pi_{Y}, \pi}^{-1}}{\longrightarrow} R \operatorname{pr}_{2 *} \operatorname{pr}_{1}^{*}\left(\pi_{\bar{X}}^{!} k\right) \\
& \rightarrow R g_{Y *} R \operatorname{pr}_{2 *}^{\prime}\left(\operatorname{pr}_{1}^{\prime}\right)^{*}\left(\pi_{\bar{X}}^{!} k\right) \\
& \stackrel{b_{\pi_{Y^{\prime}}, \pi}}{\longrightarrow} R g_{Y *} R \operatorname{pr}_{2 *}^{\prime}\left(\operatorname{pr}_{2}^{\prime}\right)^{!}\left(\pi_{Y^{\prime}}^{*} k\right)=R g_{Y *} R \operatorname{pr}_{2 *}^{\prime}\left(\operatorname{pr}_{2}^{\prime}\right)^{!} O_{Y^{\prime}},
\end{aligned}
$$

where $b_{\pi_{Y}, \pi_{\bar{X}}}: \mathrm{pr}_{1}^{*} \pi_{\bar{X}}^{!} \simeq \mathrm{pr}_{2}^{!} \pi_{Y}^{*}$ is the isomorphism from [Hartshorne 1966, Chapter VII, Corollaries 3.4(a)(5)] and the middle map is the composition of the natural maps

$R \mathrm{pr}_{2 *} \mathrm{pr}_{1}^{*} \rightarrow R g_{Y *} L g_{Y}^{*} R \mathrm{pr}_{2 *} \mathrm{pr}_{1}^{*} \rightarrow R g_{Y *} R \mathrm{pr}_{2 *}^{\prime} L g_{\bar{X} \times Y}^{*} \mathrm{pr}_{1}^{*} \cong R g_{Y *} R \mathrm{pr}_{2 *}^{\prime}\left(\mathrm{pr}_{1}^{\prime}\right)^{*}$ 
Now the commutativity of diagram (2.3.6) follows from the commutativity of

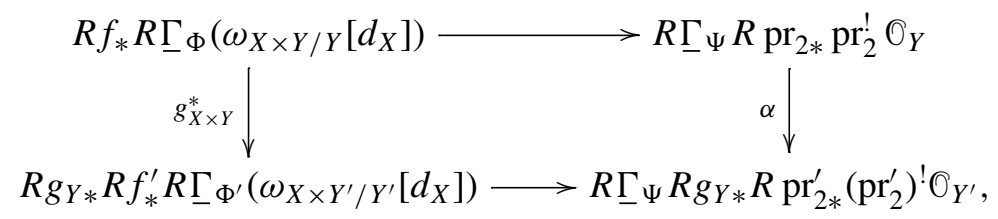

which is clear by the explicit description of the isomorphisms $b_{(\cdot, \cdot)}$ in the smooth case (see [Hartshorne 1966, Chapter VII, Corollary 3.4(a), Var 6]), and from the commutativity of the diagram

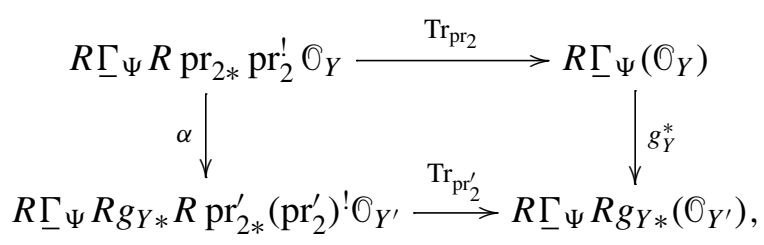

which follows from [Hartshorne 1966, Chapter VII, Corollary 3.4(b), TRA 4]. Hence the statement.

Proposition 2.3.7. Let

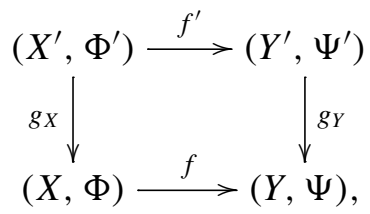

be a cartesian square with $f, f^{\prime} \in V_{*}$ and $g_{X}, g_{Y} \in V^{*}$. Assume either that $g_{Y}$ is flat or $g_{Y}$ is a closed immersion and $f$ is transversal to $Y^{\prime}$. Then

$$
H^{*}\left(g_{Y}\right) \circ H_{*}(f)=H_{*}\left(f^{\prime}\right) \circ H^{*}\left(g_{X}\right) .
$$

Proof. After embedding $X$ in $X \times Y$ via the graph morphism, the diagram above splits as

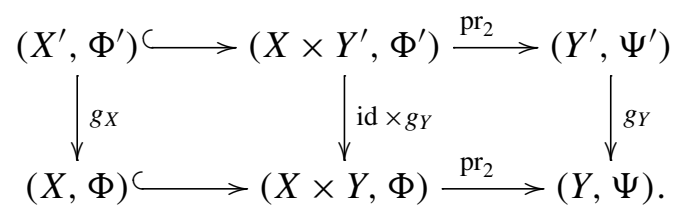

Both squares are cartesian, the projections $\mathrm{pr}_{2}$ are smooth and the inclusions are closed. If $g_{Y}$ is a closed immersion and $f$ is transversal to $Y^{\prime}$, then $\mathrm{id} \times g_{Y}$ : $X \times Y^{\prime} \rightarrow X \times Y$ is transversal to $X \hookrightarrow X \times Y$. Thus the statement follows from Proposition 2.3.3(2), Corollary 2.2.22 and Lemma 2.3.4.

Lemma 2.3.8. Let $X$ be smooth and $\imath: D \hookrightarrow X$ the inclusion of a smooth divisor. Let $\Phi$ be a family of supports on $D$ and denote by $\imath_{1}:(D, \Phi) \rightarrow(X, \Phi)$ the map in 
$V_{*}$ induced by $\mathrm{l}$. Then $H_{*}\left(l_{1}\right): H_{\Phi}^{i}\left(D, \Omega_{D}^{j}\right) \rightarrow H_{\Phi}^{i+1}\left(X, \Omega_{X}^{j+1}\right)$ is the connecting homomorphism of the long exact cohomology sequence associated to the exact sequence

$$
0 \rightarrow \Omega_{X}^{j+1} \rightarrow \Omega_{X}^{j+1}(\log D) \stackrel{\text { Res }}{\rightarrow} \imath_{*} \Omega_{D}^{j} \rightarrow 0
$$

where $\operatorname{Res}\left(\frac{d t}{t} \alpha\right)=\iota^{*}(\alpha)$ for $t \in \mathcal{O}_{X}$ a regular element defining $D$ and $\alpha \in \Omega_{X}^{j}$. In particular, if $\Phi \subset X$ is supported in codimension $\geq i+1$ in $X$, then $H_{*}\left(l_{1}\right)$ is injective on $H_{\Phi}^{i}$.

Proof. By Remark 2.2.14(1), the map $l_{*}$ from Definition 2.2.5 induces a map (also denoted by $l_{*}$ )

$$
l_{*}: \iota_{*} \Omega_{D}^{j} \rightarrow \Omega_{X}^{j+1}[1] .
$$

It suffices to show that this map coincides with the edge homomorphism coming from the distinguished triangle (2.3.9), which we denote by $\partial_{\text {Res }}$. The diagram

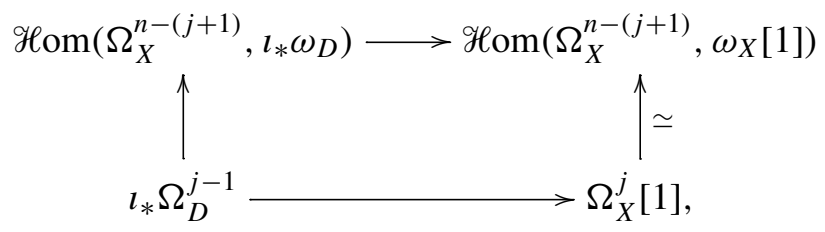

where $n=\operatorname{dim} X$ and the vertical maps are induced by multiplication from the left, is commutative for both $l_{*}$ and $\partial_{\text {Res }}$. Thus we only need to consider the case $j=n-1$.

Let $K^{\bullet}$ be the complex $\mathrm{O}_{X}(-D) \rightarrow \mathrm{O}_{X}$ in degree $[-1,0]$. Then $K^{\bullet} \rightarrow l_{*} \mathrm{O}_{D}$ is a locally free resolution. We denote by $\operatorname{Tr}_{l}^{\prime}$ the composition

$$
l_{*} \omega_{D / X}[-1] \stackrel{\eta_{l}}{\rightarrow} \imath_{*} l ! \mathrm{O}_{X} \stackrel{\operatorname{Tr}_{l}}{\rightarrow} \mathrm{O}_{X},
$$

where $\eta_{l}$ is the fundamental local isomorphism (see (A.2.1)). Then $\operatorname{Tr}_{l}^{\prime}$ is given by

$$
\operatorname{Tr}_{l}^{\prime}: l_{*} \omega_{D / X}[-1] \stackrel{\simeq}{\longleftarrow} \operatorname{Hom}^{\bullet}\left(K^{\bullet}, \mathrm{O}_{X}\right) \rightarrow \mathrm{O}_{X} .
$$

Here the first map is in degree 1 given by (see (A.2.2))

$$
\mathscr{H} \operatorname{om}\left(\mathrm{O}_{X}(-D), \mathrm{O}_{X}\right)=\mathrm{O}_{X}(D) \rightarrow t_{*} \omega_{D / X}, \quad 1 / t \mapsto-t^{\vee},
$$

where $t$ is a regular parameter defining $D$, and the second map (in degree 0 ) by Hom $\left(O_{X}, O_{X}\right)=O_{X}$. (See the proof of Lemma A.2.5 and in particular (A.2.8).)

It thus follows from the commutative diagram (A.2.14) that $\imath_{*}: l_{*} \omega_{D} \rightarrow \omega_{X}$ equals the composition

$$
l_{*} \omega_{D} \rightarrow l_{*} \omega_{D / X} \otimes \omega_{X} \stackrel{\operatorname{Tr}_{l}^{\prime}[1] \otimes \mathrm{id}}{\longrightarrow} \omega_{X}[1]
$$


where the first map is given by $\alpha \mapsto t^{\vee} \otimes(d t \wedge \tilde{\alpha})$, with $\tilde{\alpha}$ a lift. Obviously the following diagram commutes:

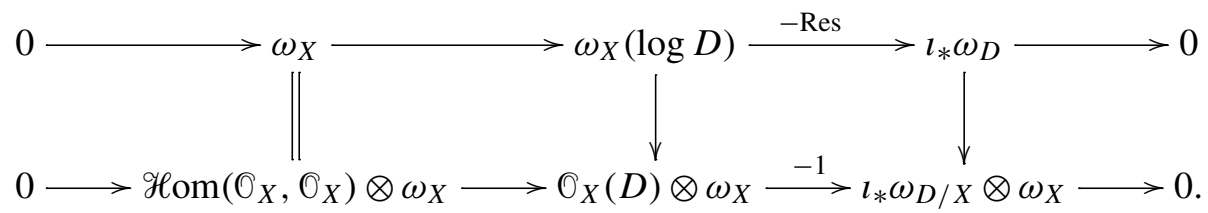

Now, by the above (and the sign conventions from [Conrad 2000, 1.3]), the map $l_{*} \omega_{D / X} \otimes \omega_{X} \rightarrow \operatorname{Hom}\left(\mathrm{O}_{X}, \mathrm{O}_{X}\right)[1] \otimes \omega_{X}$ induced by the lower exact sequence equals $-\left(\operatorname{Tr}_{l}^{\prime} \otimes\right.$ id). (Here we need that $\mathscr{H o m}^{\bullet}\left(K^{\bullet}, \mathrm{O}_{X}\right)[1]=\mathscr{H o m}\left(K^{-(\bullet+1)}, \mathscr{O}_{X}\right)$.) Thus the commutativity of the diagram above yields $i_{*}=-\partial_{- \text {Res }}=\partial_{\text {Res }}$.

2.4. The Künneth morphism. For $(X, \Phi)$ and $(Y, \Psi) \in \mathrm{ob}\left(V_{*}\right)=\mathrm{ob}\left(V^{*}\right)$, the Künneth morphism

$$
\times: H_{\Phi}^{i}\left(X, \Omega_{X}^{p}\right) \times H_{\Psi}^{j}\left(Y, \Omega_{Y}^{q}\right) \rightarrow H_{\Phi \times \Psi}^{i+j}\left(X \times Y, \Omega_{X \times Y}^{p+q}\right)
$$

is defined as the composition of the cartesian product with multiplication. Choose flasque resolutions $\Omega_{X}^{p} \rightarrow I^{\bullet}$ and $\Omega_{Y}^{q} \rightarrow J^{\bullet}$ and write $K_{\Phi}^{i}=\operatorname{Ker}\left(\Gamma_{\Phi} I^{i} \rightarrow \Gamma_{\Phi} I^{i+1}\right)$ and $K_{\Psi}^{j}=\operatorname{Ker}\left(\Gamma_{\Psi} J^{j} \rightarrow \Gamma_{\Psi} J^{j+1}\right)$. Then $\operatorname{pr}_{1}^{-1} I^{\bullet} \otimes_{k} \operatorname{pr}_{2}^{-1} J^{\bullet}$ is a resolution of $\mathrm{pr}_{1}^{-1} \Omega_{X}^{p} \otimes_{k} \mathrm{pr}_{2}^{-1} \Omega_{Y}^{q}$ and (2.4.1) is induced by the composition of the natural maps

$$
\begin{aligned}
K_{\Phi}^{i} \otimes_{k} K_{\Psi}^{j} \rightarrow & H_{\Phi \times \Psi}^{i+j}\left(\operatorname{pr}_{1}^{-1} I^{\bullet} \otimes_{k} \operatorname{pr}_{2}^{-1} J^{\bullet}\right) \\
& \rightarrow H_{\Phi \times \Psi}^{i+j}\left(X \times Y, \operatorname{pr}_{1}^{-1} \Omega_{X}^{p} \otimes_{k} \operatorname{pr}_{2}^{-1} \Omega_{Y}^{q}\right) \rightarrow H_{\Phi \times \Psi}^{i+j}\left(X \times Y, \Omega_{X \times Y}^{p+q}\right) .
\end{aligned}
$$

We define

$$
T: H(X, \Phi) \otimes H(Y, \Psi) \rightarrow H(X \times Y, \Phi \times \Psi)
$$

by the formula

$$
T\left(\alpha_{i, p} \otimes \beta_{j, q}\right)=(-1)^{(i+p) \cdot j}\left(\alpha_{i, p} \times \beta_{j, q}\right),
$$

where $\alpha_{i, p} \in H_{\Phi}^{i}\left(X, \Omega_{X}^{p}\right), \beta_{j, q} \in H_{\Psi}^{j}\left(Y, \Omega_{Y}^{q}\right)$, and $\times$ is the map in (2.4.1).

Proposition 2.4.3. The triples $\left(H_{*}, T, e\right)$ and $\left(H^{*}, T, e\right)$ define right-lax symmetric monoidal functors (see Section 1.1.9).

Lemma 2.4.4. Let $f: X \rightarrow Y$ be a morphism. Assume $Y$ to be smooth and $X$ of pure dimension $d$. Then for any $p, q \geq 0$, there is a morphism

$$
\mu: D_{X}\left(\Omega_{X}^{d-p}\right) \otimes f^{*} \Omega_{Y}^{q} \longrightarrow D_{X}\left(\Omega_{X}^{d-(p+q)}\right),
$$

such that 
(1) if $U \subset X$ is a smooth open subset, then the diagram

$$
\begin{array}{cc}
\left.D_{U}\left(\Omega_{U}^{d-p}\right)[-d] \otimes f\right|_{U} ^{*} \Omega_{Y}^{q} \stackrel{\left.\mu\right|_{U}[-d]}{\longrightarrow} D_{U}\left(\Omega_{U}^{d-(q+p)}\right)[-d] \\
(2.2 .15) \downarrow \simeq & (2.2 .15) \mid \simeq \\
\downarrow & \simeq \Omega_{U}^{p+q}
\end{array}
$$

commutes, where the lower horizontal map is given by $\alpha \otimes \beta \mapsto \alpha \wedge f^{*}(\beta)$;

(2) if $f$ is proper, then the diagram

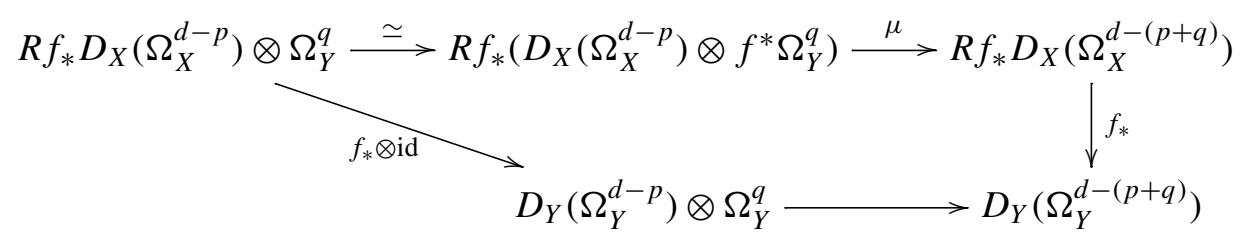

commutes, where the lower horizontal map is induced by

$\mathscr{H} \operatorname{om}\left(\Omega_{Y}^{d-p}, \Omega_{Y}^{d_{Y}}\right) \otimes \Omega_{Y}^{q} \rightarrow \operatorname{Hom}\left(\Omega_{Y}^{d-(q+p)}, \Omega_{Y}^{d_{Y}}\right), \quad \varphi \otimes \alpha \mapsto \varphi(\alpha \wedge(\cdot))$.

Proof. We denote by $\pi_{X}$ and $\pi_{Y}$ the structure maps of $X$ and $Y$, respectively. Since $\pi_{X}^{!} k$ and $\pi_{Y}^{!} k$ are dualizing complexes, they are represented by bounded complexes of injectives $I_{X}^{\bullet}$ and $I_{Y}^{\bullet}$, and $\operatorname{Tr}_{f}: f_{*} \pi_{X}^{!} k \cong f_{*} f^{!} \pi_{Y}^{!} k \rightarrow \pi_{Y}^{!} k$ is thus represented by a morphism of complexes $\operatorname{Tr}_{f}: f_{*} I_{X}^{\bullet} \rightarrow I_{Y}^{\bullet}$. Now the map

$$
\mu: \operatorname{Hom}_{X}\left(\Omega_{X}^{d-p}, I_{X}^{\bullet}\right) \otimes f^{*} \Omega_{Y}^{q} \rightarrow \operatorname{Hom}_{X}\left(\Omega_{X}^{d-(p+q)}, I_{Y}^{\bullet}\right)
$$

is in degree $n$ given by

$$
\operatorname{Hom}_{X}\left(\Omega_{X}^{d-p}, I_{X}^{n}\right) \otimes f^{*} \Omega_{Y}^{q} \rightarrow \operatorname{Hom}_{X}\left(\Omega_{X}^{d-(p+q)}, I_{Y}^{n}\right), \quad \theta \otimes \alpha \mapsto \theta\left(f^{*}(\alpha) \wedge \cdot\right) .
$$

It is immediate that this defines a map of complexes that satisfies (1). For (2) we observe that it suffices to check the commutativity of

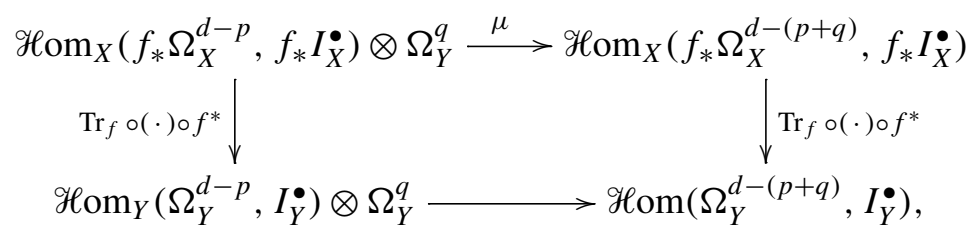

which is straightforward.

Proof of Proposition 2.4.3. Recall that $H^{*}(X, \Phi)$ is graded by $(2.0 .1)$ and $H_{*}(X, \Phi)$ is graded by (2.0.2). The morphism $T$ respects the grading for both gradings. In the following, we will work with the upper grading $H^{*}$, but all arguments will also 
work for the lower grading $H_{*}$ because the difference between lower and upper grading is an even integer.

By using the associativity of $\times$ (defined in (2.4.1)) it is straightforward to prove the associativity of $T$. Let us prove the commutativity of $T$, that is, that the diagram

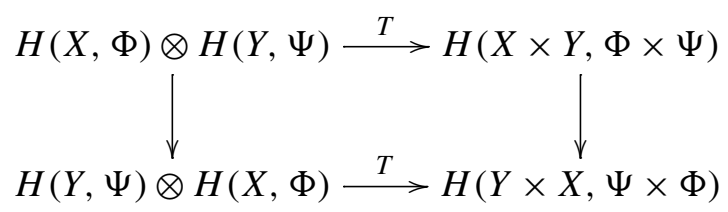

is commutative. The left vertical map is defined by $a \otimes b \mapsto(-1)^{\operatorname{deg}(a) \operatorname{deg}(b)} b \otimes a$, and the right vertical map is given by $H^{*}\left(\epsilon_{1}\right)$ and $H_{*}\left(\epsilon_{2}\right)$, respectively, with

$$
\begin{aligned}
& \epsilon_{1}:(Y \times X, \Psi \times \Phi) \rightarrow(X \times Y, \Phi \times \Psi), \\
& \epsilon_{2}:(X \times Y, \Phi \times \Psi) \rightarrow(Y \times X, \Psi \times \Phi)
\end{aligned}
$$

the obvious morphisms $\epsilon_{1} \in V^{*}$ and $\epsilon_{2} \in V_{*}$. Obviously, $H^{*}\left(\epsilon_{1}\right)=H_{*}\left(\epsilon_{2}\right)$; thus we may work with $H^{*}\left(\epsilon_{1}\right)$ in the following. Note that the diagram

$$
\begin{gathered}
H_{\Phi}^{i}\left(X, \Omega_{X}^{p}\right) \times H_{\Psi}^{j}\left(Y, \Omega_{Y}^{q}\right) \stackrel{(2.4 .1)}{\longrightarrow} H_{\Phi \times \Psi}^{i+j}\left(X \times Y, \Omega_{X \times Y}^{p+q}\right) \\
\downarrow \\
\Downarrow \\
H_{\Psi}^{j}\left(Y, \Omega_{Y}^{q}\right) \times H_{\Phi}^{i}\left(X, \Omega_{X}^{p}\right) \stackrel{(-1)^{p \cdot q} H^{*}\left(\epsilon_{1}\right)}{\longrightarrow} H_{\Psi \times \Phi}^{i+j}\left(Y \times X, \Omega_{Y \times X}^{p+q}\right)
\end{gathered}
$$

is commutative, where the left vertical arrow is defined by $a \times b \mapsto(-1)^{i \cdot j}(b \times a)$. By using this diagram it is a straightforward calculation to prove the commutativity of (2.4.5).

We still need to prove the functoriality of $T$ for $H^{*}$ and $H_{*}$. For $H^{*}$ this follows immediately from the definitions. Let us prove the functoriality for $H_{*}$. We will write $H_{\Phi}^{i}(\cdot)$ instead of $H_{\Phi}^{i}(X, \cdot)$. By using the commutativity of $T$ (that is, (2.4.5)) it is enough to prove that the diagram

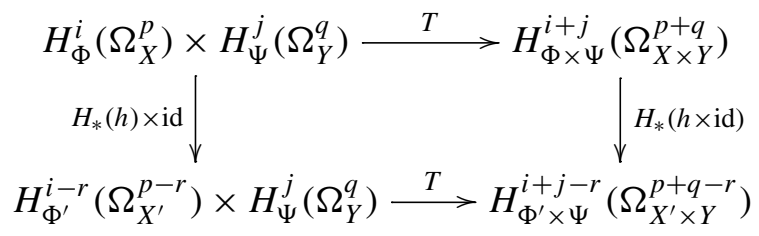

commutes for any $(Y, \Psi) \in V_{*}, h:(X, \Phi) \rightarrow\left(X^{\prime}, \Phi^{\prime}\right)$ in $V_{*}$ and $r=\operatorname{dim} X-\operatorname{dim} X^{\prime}$ ( $X$ and $X^{\prime}$ are assumed to be equidimensional). Equivalently, the diagram (2.4.6), but with $\times$ instead of $T$ as horizontal arrows, commutes. Observe that $\times$ can be 
factored as

$$
\times: H_{\Phi}^{i}\left(\Omega_{X}^{p}\right) \times H_{\Psi}^{j}\left(\Omega_{Y}^{q}\right) \stackrel{H^{*}\left(\mathrm{pr}_{1}\right) \times \mathrm{id}}{\longrightarrow} H_{\Phi \times Y}^{i}\left(\Omega_{X \times Y}^{p}\right) \times H_{\Psi}^{j}\left(\Omega_{Y}^{q}\right) \rightarrow H_{\Phi \times \Psi}^{i+j}\left(\Omega_{X \times Y}^{p+q}\right),
$$

where the map at right is the composition of the cartesian product with the multiplication map $\Omega_{X \times Y}^{p} \otimes_{k} \operatorname{pr}_{2}^{-1} \Omega_{Y}^{q} \rightarrow \Omega_{X \times Y}^{p+q}, \alpha \otimes \beta \mapsto \alpha \wedge \operatorname{pr}_{2}^{*} \beta$. By Proposition 2.3.7 the diagram

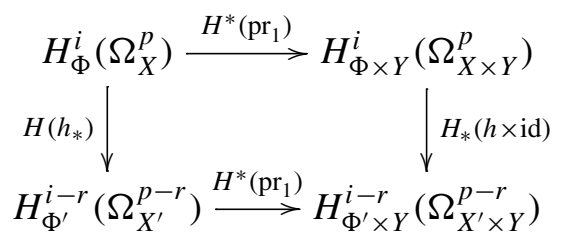

commutes. Thus it suffices to prove that

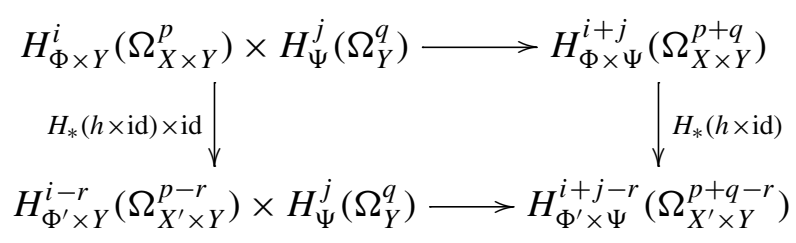

commutes.

Now let $\bar{h}: \bar{X} \rightarrow X^{\prime}$ be a compactification of $h$ and set $d=\operatorname{dim} X+\operatorname{dim} Y$. We write

$$
\omega_{\bar{X} \times Y}^{p}:=D_{\bar{X} \times Y}\left(\Omega_{\bar{X} \times Y}^{d-p}\right) \quad \text { and } \quad \omega_{X^{\prime} \times Y}^{p}:=D_{X^{\prime} \times Y}\left(\Omega_{X^{\prime} \times Y}^{d-p}\right) .
$$

Notice that $\left.\omega_{\bar{X} \times Y}^{p}\right|_{X \times Y} \cong \Omega_{X \times Y}^{p}[d]$ and $\omega_{X^{\prime} \times Y}^{p} \cong \Omega_{X^{\prime} \times Y}^{p-r}[d-r]$. With this notation the push-forward is a morphism

$$
(\bar{h} \times \mathrm{id})_{*}: R(\bar{h} \times \mathrm{id})_{*} \omega_{\bar{X} \times Y}^{p} \rightarrow \omega_{X^{\prime} \times Y}^{p}
$$

and we have to show that the following diagram commutes:

$$
\begin{array}{r}
H_{\Phi \times Y}^{i}\left(\omega_{\bar{X} \times Y}^{p}\right) \times H_{\Psi}^{j}\left(\Omega_{Y}^{p}\right) \longrightarrow H_{\Phi \times \Psi}^{i+j}\left(\omega_{\bar{X} \times Y}^{p+q}\right) \\
(h \times \text { id })_{*} \times \text { id } \downarrow \\
H_{\Phi^{\prime} \times Y}^{i}\left(\omega_{X^{\prime} \times Y}^{p}\right) \times H_{\Psi}^{j}\left(\Omega_{Y}^{p}\right) \\
(h \times i d)_{*}
\end{array}
$$

where the upper map is given by the cartesian product composed with the $\mu$ from Lemma 2.4.4. Clearly we may assume $\Phi=\bar{h}^{-1}\left(\Phi^{\prime}\right)$; thus

$$
H_{\Phi \times Y}^{i}\left(\omega_{\bar{X} \times Y}^{p}\right)=H_{\Phi^{\prime} \times Y}^{i}\left(R(\bar{h} \times \mathrm{id})_{*} \omega_{\bar{X} \times Y}^{p}\right) .
$$


Now it follows from Lemma 2.4.4(2) that it is enough to prove the commutativity of the diagram

$$
\begin{aligned}
& H_{\Phi \times Y}^{i}\left(\omega_{\bar{X} \times Y}^{p}\right) \times H_{\Psi}^{j}\left(\Omega_{Y}^{q}\right) \longrightarrow H_{\Phi^{\prime} \times \Psi}^{i+j}\left(R(\bar{h} \times \mathrm{id})_{*}\left(\omega_{\bar{X} \times Y}^{p}\right) \otimes_{k} \operatorname{pr}_{2}^{-1} \Omega_{Y}^{q}\right) \\
& (\bar{h} \times \text { id })_{*} \times \text { id } \downarrow \quad \downarrow(\bar{h} \times \text { id })_{*} \otimes \text { id } \\
& H_{\Phi^{\prime} \times Y}^{i}\left(\omega_{X^{\prime} \times Y}^{p}\right) \times H_{\Psi}^{j}\left(\Omega_{Y}^{q}\right) \longrightarrow H_{\Phi^{\prime} \times \Psi}^{i+j}\left(\omega_{X^{\prime} \times Y}^{p} \otimes_{k} \operatorname{pr}_{2}^{-1} \Omega_{Y}^{q}\right),
\end{aligned}
$$

where the upper horizontal map is the composition of (2.4.8) with the cartesian product, and the diagram

$$
\begin{aligned}
& H_{\Phi^{\prime} \times \Psi}^{i+j}\left(R(\bar{h} \times \mathrm{id})_{*}\left(\omega_{\bar{X} \times Y}^{p}\right) \otimes_{k} \operatorname{pr}_{2}^{-1} \Omega_{Y}^{q}\right) \longrightarrow H_{\Phi^{\prime} \times \Psi}^{i+j}\left(R(\bar{h} \times \mathrm{id})_{*}\left(\omega_{\bar{X} \times Y}^{p}\right) \otimes_{\odot} \operatorname{pr}_{2}^{*} \Omega_{Y}^{q}\right) \\
& (\bar{h} \times \mathrm{id})_{*} \otimes \mathrm{id} \downarrow \downarrow \downarrow(\bar{h} \times \mathrm{id})_{*} \otimes \mathrm{id} \\
& H_{\Phi^{\prime} \times \Psi}^{i+j}\left(\omega_{X^{\prime} \times Y}^{p} \otimes_{k} \operatorname{pr}_{2}^{-1} \Omega_{Y}^{q}\right) \longrightarrow H_{\Phi^{\prime} \times \Psi}^{i+j}\left(\omega_{X^{\prime} \times Y}^{p} \otimes_{\mathcal{O}} \operatorname{pr}_{2}^{*} \Omega_{Y}^{q}\right) .
\end{aligned}
$$

For this take injective resolutions $\omega_{\bar{X} \times Y}^{p} \rightarrow I^{\bullet}$ and $\omega_{X^{\prime} \times Y}^{p} \rightarrow J^{\bullet}$; then the pushforward is given by an actual morphism $(\bar{h} \times \mathrm{id})_{*} I^{\bullet} \rightarrow J^{\bullet}$. Now the commutativity of the first diagram is easily checked by taking an injective resolution of $\Omega_{Y}^{q}$. For the commutativity of the second we observe that

$$
(\bar{h} \times \mathrm{id})_{*} I^{\bullet} \otimes_{k} \operatorname{pr}_{2}^{-1} \Omega_{Y}^{q} \quad \text { and } \quad(\bar{h} \times \mathrm{id})_{*} I^{\bullet} \otimes_{0} \operatorname{pr}_{2}^{*} \Omega_{Y}^{q}
$$

still represent

$$
R(\bar{h} \times \mathrm{id})_{*} \omega_{\bar{X} \times Y}^{p} \otimes_{k} \operatorname{pr}_{2}^{-1} \Omega_{Y}^{q} \quad \text { and } \quad R(\bar{h} \times \mathrm{id})_{*} \omega_{\bar{X} \times Y}^{p} \otimes_{\odot} \operatorname{pr}_{2}^{*} \Omega_{Y}^{q},
$$

respectively, and similarly with $(\bar{h} \times \mathrm{id})_{*} I^{\bullet}$ replaced by $J^{\bullet}$ and $(\bar{h} \times \mathrm{id})_{*} \omega_{\bar{X} \times Y}^{p}$ by $\omega_{X^{\prime} \times Y}^{p}$. Thus it is enough to check the commutativity using these complexes, which is obvious.

2.5. Summary. Let $\left(H_{*}, H^{*}, T, e\right)$ be the datum defined above, that is, we define $H_{*}: V_{*} \rightarrow \mathbf{G r A b}$ on objects by (2.0.2) and on morphisms by Definition 2.3.2, we define $H^{*}:\left(V^{*}\right)^{\text {op }} \rightarrow \mathbf{G r A b}$ on objects by (2.0.1) and on morphisms by (2.1.9), and we define $T$ by (2.4.2) and $e$ by (2.0.3).

Theorem 2.5.1. The datum $\left(H_{*}, H^{*}, T, e\right)$ is an object in $\mathbf{T}$, that is, it is a datum as in 1.1.8 and satisfies the properties 1.1.9.

We denote by $H P$ the pure part of $H$, that is,

$$
H P(X, \Phi):=\bigoplus_{n \geq 0} H_{\Phi}^{n}\left(X, \Omega_{X}^{n}\right) \quad \text { for }(X, \Phi) \in V_{*},
$$


and let $H P^{*}(X, \Phi)$ be the graded abelian group, which in degree $2 n$ equals

$$
H P^{2 n}(X, \Phi)=H_{\Phi}^{n}\left(X, \Omega_{X}^{n}\right)
$$

and is zero in odd degrees. The graded abelian group $H P_{*}(X, \Phi)$ is defined as in (2.0.2). Then the functors $H_{*}$ and $H^{*}$ induce functors $H P_{*}$ and $H P^{*}$, and $T$ and $e$ restrict to $H P$.

Corollary 2.5.3. The datum $\left(H P_{*}, H P^{*}, T, e\right)$ is an object in $\mathbf{T}$. Furthermore $H P$ satisfies the semipurity condition from Definition 1.2.1 and the natural map $\left(H P_{*}, H P^{*}, T, e\right) \rightarrow\left(H_{*}, H^{*}, T, e\right)$ is a morphism in $\mathbf{T}$.

Proof. The semipurity condition follows from [Grothendieck 1968, Exposé III, Proposition 3.3].

\section{Cycle class map to Hodge cohomology and applications}

In this section $k$ is assumed to be a perfect field (unless stated otherwise).

\subsection{Cycle class.}

Proposition 3.1.1 (cycle class). Let $X$ be a smooth scheme and let $W \subset X$ be an irreducible closed subset of codimension $c$. There is a class $c l_{(X, W)}=c l(W) \in$ $H_{W}^{c}\left(X, \Omega_{X}^{c}\right)$ with the property that

$$
H^{*}(\jmath)(c l(W))=H_{*}\left(l_{U \cap W}\right)(1)
$$

for every open subset $U \subset X$ such that $U \cap W$ is smooth (and nonempty), where $J:(U, W \cap U) \rightarrow(X, W)$ and $\imath: W \cap U \rightarrow(U, W \cap U)$ are induced by the open and closed immersion, respectively, and 1 is the identity element of the ring $H^{0}\left(X, \mathrm{O}_{X}\right)$.

Remark 3.1.2. The cycle class in the proposition is Grothendieck's "fundamental class"; see for example [Lipman 1984, page 39, (ii)]. For the convenience of the reader and to be sure about the compatibility with the push-forward constructed in the previous section, we give a proof of the proposition, which is standard.

Proof. 1st step: Let $\eta$ be the generic point of $W$. We define

$$
H_{\eta}^{c}\left(X, \Omega_{X}^{c}\right):=\underset{\eta \in U}{\lim _{U}} H_{U \cap W}^{c}\left(U, \Omega_{U}^{c}\right),
$$

where the inductive limit runs over all open sets $U \subset X$ with $\eta \in U$. Choose $U$ such that $U \cap W \neq \varnothing$ is smooth. The image of $H_{*}\left(l_{U \cap W}\right)(1) \in H_{U \cap W}^{c}\left(U, \Omega_{U}^{c}\right)$ in $H_{\eta}^{c}\left(X, \Omega_{X}^{c}\right)$ doesn't depend on the choice of $U$ by Section 1.1.9(4). We denote this class by $c l(W)_{\eta}$. 
2nd step: A class $a \in H_{\eta}^{c}\left(X, \Omega_{X}^{c}\right)$ is in the image of

$$
H_{W}^{c}\left(X, \Omega_{X}^{c}\right) \rightarrow H_{\eta}^{c}\left(X, \Omega_{X}^{c}\right)
$$

(that is, extends to a global class) if and only if for all 1-codimensional points $x$ in $W$ there is an open subset $U \subset X$ containing $x$, so that $a$ lies in the image of

$$
H_{W \cap U}^{c}\left(U, \Omega_{U}^{c}\right) \rightarrow H_{\eta}^{c}\left(X, \Omega_{X}^{c}\right) .
$$

Indeed, the Cousin resolution yields an exact sequence

$$
0 \rightarrow H_{W}^{c}\left(X, \Omega_{X}^{c}\right) \rightarrow H_{\eta}^{c}\left(X, \Omega_{X}^{c}\right) \rightarrow \bigoplus_{x \in W, c d(x)=1} H_{x}^{c+1}\left(X, \Omega_{X}^{c}\right),
$$

and $H_{U \cap W}^{c}\left(U, \Omega_{U}^{c}\right) \rightarrow H_{\eta}^{c}\left(X, \Omega_{X}^{c}\right) \rightarrow H_{x}^{c+1}\left(X, \Omega_{X}^{c}\right)$ vanishes for all $x$ and $U$ as above.

3rd step: If $W$ is normal then $\operatorname{cl}(W)_{\eta}$ extends (uniquely) to a class in $H_{W}^{c}\left(X, \Omega_{X}^{c}\right)$. Indeed, since $W$ is regular in codimension one and we assume that $k$ is perfect, we may choose an open $U \subset X$ such that $U \cap W$ is smooth and $U \cap W$ contains all points of codimension 1 of $W$. So that the class extends by the 2 nd step. Note that the extension is unique because of the exact sequence (3.1.3).

4th step: We claim that the class $c l(W)_{\eta}$ extends to a class in $H_{W}^{c}\left(X, \Omega_{X}^{c}\right)$. In view of the 2nd step it is sufficient to extend the class at all points $x \in W$ of codimension 1. Thus we may assume that $X$ (and therefore $W$ ) is affine. The normalization $\tilde{W} \rightarrow W$ is a finite morphism and thus projective. Choose an embedding $\tilde{W} \rightarrow$ $W \times{ }_{k} \mathbb{P}_{k}^{n}$ over $W$. The previous step yields a class $c l(\tilde{W}) \in H_{\tilde{W}}^{n+c}\left(X \times \mathbb{P}^{n}, \Omega_{X \times \mathbb{P}^{n}}^{n+c}\right)$. Consider $H_{*}\left(\operatorname{pr}_{1}\right)(c l(\tilde{W})) \in H_{W}^{c}\left(X, \Omega_{X}^{c}\right)$; for an open $U \subset X$ such that $W \cap U \neq \varnothing$ and $U \cap W$ is smooth, we obtain

$$
\begin{aligned}
H^{*}(j) H_{*}\left(\operatorname{pr}_{1}\right)(c l(\tilde{W})) & =H_{*}\left(\operatorname{pr}_{1 \mid U \times \mathbb{P}^{n}}\right) H^{*}\left(J^{\prime}\right)(\operatorname{cl}(\tilde{W})) \\
& =H_{*}\left(\operatorname{pr}_{1 \mid U \times \mathbb{P}^{n}}\right) H_{*}\left(l_{\left(U \times \mathbb{P}^{n}\right) \cap \tilde{W}}\right)(1)=H_{*}\left(l_{U \cap W}\right)(1),
\end{aligned}
$$

with $\jmath^{\prime}:\left(U \times \mathbb{P}^{n},\left(U \times \mathbb{P}^{n}\right) \cap \tilde{W}\right) \rightarrow\left(X \times \mathbb{P}^{n}, \tilde{W}\right)$. Thus $H_{*}\left(\operatorname{pr}_{1}\right)(\operatorname{cl}(\tilde{W}))$ is the desired lift.

3.1.4. Explicit description of the cycle class. Let $X$ be a smooth scheme and let $W \subset X$ be an irreducible closed subset of codimension $c$ with generic point $\eta \in X$. Denote $A=O_{X, \eta}$. Then

$$
H_{\eta}^{c}\left(X, \Omega_{X}^{c}\right)=\underset{f \subset \mathfrak{m}_{\eta}}{\lim } \frac{\Omega_{A}^{c}}{(f)},
$$

where the limit is over all $A$-sequences $f=\left(f_{1}, \ldots, f_{c}\right)$ of length $c$ that are contained in $\mathfrak{m}_{\eta}$ (in particular $\left.\sqrt{\left(f_{1}, \ldots, f_{c}\right)}=\mathfrak{m}_{\eta}\right)$. The class of $\omega \in \Omega_{A}^{c}$ under the 
composition in $\Omega_{A}^{c} \rightarrow \Omega_{A}^{c} /(f) \rightarrow H_{\eta}^{c}\left(X, \Omega_{X}^{c}\right)$ is denoted by $\left[\begin{array}{c}\omega \\ f\end{array}\right]$. See Section A.1 for details.

Now let $U$ be an affine open subset of $X$ such that $U \cap W$ is smooth and the ideal of $W \cap U$ in $O_{U}$ is generated by global sections $t_{1}, \ldots, t_{c}$ on $U$. Then by Proposition 2.2.19

$$
c l(W)_{\eta}=(-1)^{c}\left[\begin{array}{c}
d t_{1} \cdots d t_{c} \\
t_{1}, \ldots, t_{c}
\end{array}\right] .
$$

Lemma 3.1.5. For a closed immersion $\imath: X \rightarrow Y$ between smooth schemes and an effective smooth divisor $D \subset Y$ such that

- D meets $X$ properly, and thus $D \cap X:=D \times_{Y} X$ is a divisor on $X$,

- $D^{\prime}:=(D \cap X)_{\text {red }}$ is smooth and connected, and thus $D \cap X=n \cdot D^{\prime}$ as divisors (for some $n \in \mathbb{Z}$ with $n \geq 1$ ),

we denote by $\iota_{X}: X \rightarrow(Y, X)$ and $\iota_{D^{\prime}}: D^{\prime} \rightarrow\left(D, D^{\prime}\right)$ the morphisms in $V_{*}$ induced by $l$, and we define $g_{2}:\left(D, D^{\prime}\right) \rightarrow(Y, X)$ in $V^{*}$ by the inclusion $g: D \hookrightarrow Y$. Then the following equality holds:

$$
H^{*}\left(g_{2}\right)\left(H_{*}\left(l_{X}\right)\left(1_{X}\right)\right)=n \cdot H_{*}\left(l_{D^{\prime}}\right)\left(1_{D^{\prime}}\right) .
$$

Proof. Let $c$ be the codimension of $X$ in $Y$ and $g_{3}:\left(D, D^{\prime}\right) \rightarrow\left(Y, D^{\prime}\right)$ be induced by the inclusion $D \subset Y$. Then

$$
H_{*}\left(g_{3}\right): H_{D^{\prime}}^{c}\left(D, \Omega_{D}^{c}\right) \rightarrow H_{D^{\prime}}^{c+1}\left(Y, \Omega_{Y}^{c+1}\right)
$$

is injective (by Lemma 2.3.8), and thus we need to prove

$$
H_{*}\left(g_{3}\right) H^{*}\left(g_{2}\right) H_{*}\left(l_{X}\right)\left(1_{X}\right)=n \cdot H_{*}\left(g_{3}\right) H_{*}\left(l_{D^{\prime}}\right)\left(1_{D^{\prime}}\right) .
$$

Let $g_{1}: D \rightarrow(Y, D)$ be induced by $g$; then projection formula 1.1 .16 gives

$$
H_{*}\left(g_{3}\right) H^{*}\left(g_{2}\right)\left(H_{*}\left(l_{X}\right)\left(1_{X}\right)\right)=H_{*}\left(g_{1}\right)\left(1_{D}\right) \cup H_{*}\left(l_{X}\right)\left(1_{X}\right) .
$$

Therefore it suffices to prove

$$
H_{*}\left(g_{1}\right)\left(1_{D}\right) \cup H_{*}\left(l_{X}\right)\left(1_{X}\right)=n \cdot H_{*}\left(g_{3} \circ l_{D^{\prime}}\right)\left(1_{D^{\prime}}\right) .
$$

Let $\eta$ be the generic point of $D^{\prime}$. Since $H_{Z}^{c+1}\left(Y, \Omega_{Y}^{c+1}\right)=0$ for all closed subsets $Z \subset Y$ of codimension $\geq c+2$, by [Grothendieck 1968, Exposé III, Proposition 3.3], the restriction map

$$
H_{D^{\prime}}^{c+1}\left(Y, \Omega_{Y}^{c+1}\right) \rightarrow H_{\eta}^{c+1}\left(Y, \Omega_{Y}^{c+1}\right)
$$

is injective. Thus it is sufficient to prove the equality (3.1.6) in $H_{\eta}^{c+1}\left(Y, \Omega_{Y}^{c+1}\right)$. 
Since $X$ is smooth we may find a regular sequence $t_{1}, \ldots, t_{c} \in \mathcal{O}_{Y, \eta}$ that generates the ideal of $X$. If $D=\operatorname{div}(f)$ around $\eta$, then

$$
(-1)\left[\begin{array}{c}
d f \\
f
\end{array}\right] \cup(-1)^{c}\left[\begin{array}{c}
d t_{1} \wedge \cdots \wedge d t_{c} \\
t_{1}, \ldots, t_{c}
\end{array}\right]=(-1)^{c+1}\left[\begin{array}{c}
d f \wedge d t_{1} \wedge \cdots \wedge d t_{c} \\
f, t_{1}, \ldots, t_{c}
\end{array}\right]
$$

is the image of $H_{*}\left(g_{1}\right)\left(1_{D}\right) \cup H_{*}\left(l_{X}\right)\left(1_{X}\right)$ in $H_{\eta}^{c+1}\left(Y, \Omega_{Y}^{c+1}\right)$.

Let $\pi \in \mathscr{O}_{Y, \eta}$ be a lift of a generator of the maximal ideal in $\mathscr{O}_{X, \eta}$. By the explicit description of the cycle class in Section 3.1.4 we get

$$
H_{*}\left(g_{3} \circ l_{D^{\prime}}\right)\left(1_{D^{\prime}}\right)=(-1)^{c+1}\left[\begin{array}{c}
d \pi \wedge d t_{1} \wedge \cdots \wedge d t_{c} \\
\pi, t_{1}, \ldots, t_{c}
\end{array}\right] .
$$

Obviously $f=a \pi^{n}$ in $\mathcal{O}_{X, \eta}$ for a unit $a \in \mathcal{O}_{X, \eta}^{*}$. Choose a lift $\tilde{a} \in \mathcal{O}_{Y, \eta}^{*}$ of $a$; thus $f=\tilde{a} \pi^{n}$ modulo $\left(t_{1}, \ldots, t_{c}\right)$, and we obtain

$$
\begin{aligned}
{\left[\begin{array}{c}
d f \wedge d t_{1} \wedge \cdots \wedge d t_{c} \\
f, t_{1}, \ldots, t_{c}
\end{array}\right] } & =\left[\begin{array}{c}
n \tilde{a} \pi^{n-1} \cdot d \pi \wedge d t_{1} \wedge \cdots \wedge d t_{c} \\
\tilde{a} \pi^{n}, t_{1}, \ldots, t_{c}
\end{array}\right] \\
& =n \cdot\left[\begin{array}{c}
d \pi \wedge d t_{1} \wedge \cdots \wedge d t_{c} \\
\pi, t_{1}, \ldots, t_{c}
\end{array}\right],
\end{aligned}
$$

which proves (3.1.6).

Theorem 3.1.8. There exists a morphism $\mathrm{cl}: \mathrm{CH} \rightarrow H=\left(H_{*}, H^{*}, T, e\right)$ in $\mathbf{T}$.

Proof. Since there is a morphism $H P=\left(H P_{*}, H P^{*}, T, e\right) \rightarrow H$ in $\mathbf{T}$, it suffices to prove the existence of $\mathrm{cl}: \mathrm{CH} \rightarrow \mathrm{HP}$.

This follows from Theorem 1.2.3, since $H P$ satisfies all the conditions listed there: $H P$ is in $\mathbf{T}$ and satisfies the semipurity condition 1.2 .1 by Corollary 2.5.3. It satisfies 1.2.3(1) by Proposition 2.3.3(3) and 1.2.3(3) by Lemma 3.1.5. Finally the element $c l_{(X, W)}$ from 1.2.3(4) is the cycle class constructed in Proposition 3.1.1 and 1.2.3(2) is obvious.

\subsection{Main theorems.}

3.2.1. Let $f:(X, \Phi) \rightarrow(Y, \Psi)$ be a morphism in $V_{*}$ or $V^{*}$. By Theorem 3.1.8, Section 1.3.18 and Lemma 1.3.19, the morphisms

$$
H_{*}(f): H_{*}(X, \Phi) \rightarrow H_{*}(Y, \Psi) \quad \text { and } \quad H^{*}(f): H^{*}(Y, \Psi) \rightarrow H^{*}(X, \Phi)
$$

are respectively given by (we write $c l$ instead of $\operatorname{Cor}(c l)$ )

$$
H_{*}(f)=\rho_{H} \circ c l \circ \tau_{*}^{\mathrm{CH}}(f) \text { and } H^{*}(f)=\rho_{H} \circ c l \circ \tau_{\mathrm{CH}}^{*}(f) .
$$

Thus we may use composition of correspondences in $\mathrm{Cor}_{\mathrm{CH}}$ to compute $H^{*}(f) \circ$ $H_{*}(f), H_{*}(f) \circ H^{*}(f)$, etc. 
Proposition 3.2.2. Let $X, Y$ be smooth and connected, and let

$$
\alpha \in \operatorname{Hom}_{\mathrm{Cor}_{C H}}(X, Y)^{0}=C H^{\operatorname{dim} X}\left(X \times Y, P\left(\Phi_{X}, \Phi_{Y}\right)\right) .
$$

(1) If the support of $\alpha$ projects to an $r$-codimensional subset in $Y$, then the restriction of $\rho_{H} \circ \operatorname{cl}(\alpha)$ to $\bigoplus_{j<r, i} H^{i}\left(X, \Omega_{X}^{j}\right)$ vanishes.

(2) If the support of $\alpha$ projects to an $r$-codimensional subscheme in $X$, then the restriction of $\rho_{H} \circ \operatorname{cl}(\alpha)$ to $\bigoplus_{j \geq \operatorname{dim} X-r+1, i} H^{i}\left(X, \Omega_{X}^{j}\right)$ vanishes.

Proof. (1) We may assume $\alpha=[V]$ for $V \subset X \times Y$ a closed irreducible subset of dimension $\operatorname{dim}(Y)=: d_{Y}$, with $p_{Y}(V) \subset Y$ of codimension $r$. We set $d_{X}=\operatorname{dim} X$.

By definition of $\rho_{H}$ (see Section 1.3.18) and Lemma 2.3.4 it is sufficient to prove that for all $0 \leq q \leq r-1$ the image of the class $c l(V)$ vanishes via the map

$$
H_{V}^{d_{X}}\left(\Omega_{X \times Y}^{d_{X}}\right) \stackrel{\text { proj. }}{\longrightarrow} H_{V}^{d_{X}}\left(\operatorname{pr}_{1}^{*} \Omega_{X}^{d_{X}-q} \otimes \mathrm{pr}_{2}^{*} \Omega_{Y}^{q}\right) .
$$

To prove this we may also localize at the generic point $\eta$ of $V$ [Grothendieck 1968, Exposé III, Proposition 3.3].

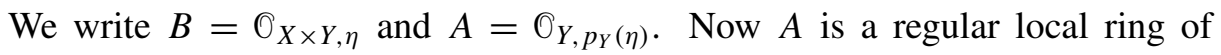
dimension $r$ and $B$ is formally smooth over $A$. Let $t_{1}, \ldots, t_{r} \in A$ be a regular system of parameters of $A$. Since $B /\left(1 \otimes t_{1}, \ldots, 1 \otimes t_{r}\right)$ is a local regular ring there exist elements $s_{r+1}, \ldots, s_{d_{X}} \in B$ such that $1 \otimes t_{1}, \ldots, 1 \otimes t_{r}, s_{r+1}, \ldots, s_{d_{X}}$ is a system of regular parameters for $B$. Thus by the explicit description of the cycle class in Section 3.1.4 we obtain

$$
c l(V)_{\eta}=(-1)^{d_{X}}\left[\begin{array}{c}
d\left(1 \otimes t_{1}\right) \wedge \cdots \wedge d\left(1 \otimes t_{r}\right) \wedge d s_{r+1} \wedge \cdots \wedge d s_{d_{X}} \\
1 \otimes t_{1}, \ldots, 1 \otimes t_{r}, s_{r+1}, \ldots, s_{d_{X}}
\end{array}\right] .
$$

This clearly implies the claim.

(2) Let $\alpha=[V]$ be as in (1) and suppose $p_{X}(V)$ has codimension $r$ in $X$. As above it suffices to prove that for all $0 \leq q \leq r-1$ the image of the class $\operatorname{cl}(V)$ vanishes under the projection map

$$
H_{V}^{d_{X}}\left(\Omega_{X \times Y}^{d_{X}}\right) \stackrel{\text { proj. }}{\longrightarrow} H_{V}^{d_{X}}\left(\operatorname{pr}_{1}^{*} \Omega_{X}^{q} \otimes \operatorname{pr}_{2}^{*} \Omega_{Y}^{d_{X}-q}\right) .
$$

Write $C=\mathcal{O}_{X, p_{X}(\eta)}$. Then as in (1) we find $\tau_{1}, \ldots, \tau_{r} \in C$ and $\sigma_{r+1}, \ldots, \sigma_{d_{X}} \in B$, such that $\tau_{1} \otimes 1, \ldots, \tau_{r} \otimes 1, \sigma_{r+1}, \ldots, \sigma_{d_{X}}$ is a system of regular parameters for $B$. Thus

$$
c l(V)_{\eta}=(-1)^{d_{X}}\left[\begin{array}{c}
d\left(\tau_{1} \otimes 1\right) \wedge \cdots \wedge d\left(\tau_{r} \otimes 1\right) \wedge d \sigma_{r+1} \wedge \cdots \wedge d \sigma_{d_{X}} \\
\tau_{1} \otimes 1, \ldots, \tau_{r} \otimes 1, \sigma_{r+1}, \ldots, \sigma_{d_{X}}
\end{array}\right],
$$

which implies the claim. 
3.2.3. Let $S$ be a $k$-scheme and let $f: X \rightarrow S$ and $g: Y \rightarrow S$ be two integral $S$-schemes that are smooth over $k$. Let $Z \subset X \times{ }_{S} Y$ be a closed integral subscheme of dimension equal to the dimension of $Y$ and such that $\operatorname{pr}_{2} \mid Z: Z \rightarrow Y$ is proper. For an open subset $U \subset S$, we denote by $Z_{U} \subset f^{-1}(U) \times_{U} g^{-1}(U)$ the pullback of $Z$ over $U$. This gives a correspondence $\left[Z_{U}\right] \in \operatorname{Hom}_{\mathrm{Cor} C \mathrm{CH}}\left(f^{-1}(U), g^{-1}(U)\right)^{0}$, which induces a morphism of $k$-vector spaces

$$
\rho_{H} \circ \operatorname{cl}\left(\left[Z_{U}\right]\right): H^{i}\left(f^{-1}(U), \Omega_{f^{-1}(U)}^{j}\right) \rightarrow H^{i}\left(g^{-1}(U), \Omega_{g^{-1}(U)}^{j}\right) \quad \text { for all } i, j .
$$

Proposition 3.2.4. In the situation above, the set $\left\{\rho_{H} \circ \operatorname{cl}\left(\left[Z_{U}\right]\right) \mid U \subset Z\right.$ open $\}$ induces a morphism of quasicoherent $\mathrm{O}_{S}$-modules

$$
\rho_{H}(Z / S): R^{i} f_{*} \Omega_{X}^{j} \rightarrow R^{i} g_{*} \Omega_{Y}^{j} \quad \text { for all } i, j .
$$

Proof. We need to show the following statements:

(1) The maps $\rho_{H} \circ \operatorname{cl}\left(\left[Z_{U}\right]\right)$ are compatible with restriction to open sets.

(2) The maps $\rho_{H} \circ \operatorname{cl}\left(\left[Z_{U}\right]\right)$ are $\mathcal{O}(U)$-linear.

To show (1), let us denote by

$$
\begin{aligned}
& \operatorname{pr}_{1, U}: f^{-1}(U) \times g^{-1}(U) \rightarrow f^{-1}(U) \text { for } \operatorname{pr}_{1, U} \in V^{*}, \\
& \operatorname{pr}_{2, U}:\left(f^{-1}(U) \times g^{-1}(U), P\left(\Phi_{f^{-1}(U)}, \Phi_{g^{-1}(U)}\right) \rightarrow g^{-1}(U) \text { for } \operatorname{pr}_{2, U} \in V_{*},\right.
\end{aligned}
$$

the morphism induced by the projections (see (1.1.2) and (1.3.8) for the definition of $\left.P\left(\Phi_{f^{-1}(U)}, \Phi_{g^{-1}(U)}\right)\right)$. Let $j: V \hookrightarrow U$ be an open immersion and denote by

$$
j_{f}: f^{-1}(V) \rightarrow f^{-1}(U) \quad \text { and } \quad j_{g}: g^{-1}(V) \rightarrow g^{-1}(U)
$$

the morphisms in $V^{*}$ induced by $j$.

We have to show that for all $a \in H^{i}\left(f^{-1}(U), \Omega_{f^{-1}(U)}^{j}\right)$

$$
\begin{aligned}
H^{*}\left(j_{g}\right) H_{*}\left(\operatorname{pr}_{2, U}\right)\left(H^{*}\left(\operatorname{pr}_{1, U}\right)(a) \cup \operatorname{cl}\left(\left[Z_{U}\right]\right)\right) \\
=H_{*}\left(\operatorname{pr}_{2, V}\right)\left(H^{*}\left(\operatorname{pr}_{1, V}\right)\left(H^{*}\left(j_{f}\right)(a) \cup \operatorname{cl}\left(\left[Z_{V}\right]\right)\right) .\right.
\end{aligned}
$$

As a first step from the left side to the right side we observe that

$$
H^{*}\left(j_{g}\right) H_{*}\left(\mathrm{pr}_{2, U}\right)=H_{*}\left(\mathrm{pr}_{2, V}^{\prime}\right) H^{*}\left(\operatorname{id}_{f^{-1}(U)} \times j_{g}\right),
$$

where $\operatorname{pr}_{2, V}^{\prime}:\left(f^{-1}(U) \times g^{-1}(V), \Phi\right) \rightarrow g^{-1}(V)$ in $V_{*}$ is induced by the projection and $\Phi:=\left(\mathrm{id} \times j_{g}\right)^{-1} P\left(\Phi_{f^{-1}(U)}, \Phi_{g^{-1}(U)}\right)$. Denoting $\operatorname{pr}_{1, U}^{\prime}: f^{-1}(U) \times g^{-1}(V) \rightarrow$ $f^{-1}(U)$ as a morphism in $V^{*}$, we obtain the equality

$$
H^{*}\left(\operatorname{id}_{f^{-1}(U)} \times j_{g}\right)\left(H^{*}\left(\operatorname{pr}_{1, U}\right)(a) \cup \operatorname{cl}\left(\left[Z_{U}\right]\right)\right)=H^{*}\left(\operatorname{pr}_{1, U}^{\prime}\right)(a) \cup \operatorname{cl}\left(\left[Z_{V}\right]\right)
$$


in $H\left(f^{-1}(U) \times g^{-1}(V), \Phi\right)$; here we consider $Z_{V} \in \Phi$ as a closed subset of $f^{-1}(U) \times g^{-1}(V)$. Next, consider the morphisms

$$
\begin{aligned}
j_{f} \times \operatorname{id}_{g^{-1}(V)}: f^{-1}(V) \times g^{-1}(V) & \rightarrow f^{-1}(U) \times g^{-1}(V), \\
\tau:\left(f^{-1}(V) \times g^{-1}(V), Z_{V}\right) & \rightarrow\left(f^{-1}(U) \times g^{-1}(V), \Phi\right), \\
\operatorname{id}^{\prime}:\left(f^{-1}(V) \times g^{-1}(V), Z_{V}\right) & \rightarrow\left(f^{-1}(V) \times g^{-1}(V), P\left(\Phi_{f^{-1}(V)}, \Phi_{g^{-1}(V)}\right)\right),
\end{aligned}
$$

with

$$
j_{f} \times \operatorname{id}_{g^{-1}(V)} \in V^{*}, \quad \tau \in V_{*}, \quad \operatorname{id}^{\prime} \in V_{*},
$$

and where $\mathrm{id}^{\prime}$ is induced by the identity. The projection formula yields

$$
\begin{aligned}
H^{*}\left(\operatorname{pr}_{1, U}^{\prime}\right)(a) \cup \operatorname{cl}\left(\left[Z_{V}\right]\right) & =H^{*}\left(\operatorname{pr}_{1, U}^{\prime}\right)(a) \cup \operatorname{cl}\left(\mathrm{CH}_{*}(\tau)\left(\left[Z_{V}\right]\right)\right) \\
& =H_{*}(\tau)\left(H^{*}\left(j_{f} \times \operatorname{id}_{g^{-1}(V)}\right) H^{*}\left(\operatorname{pr}_{1, U}^{\prime}\right)(a) \cup \operatorname{cl}\left(\left[Z_{V}\right]\right)\right) .
\end{aligned}
$$

Now the equalities

$$
\begin{aligned}
H_{*}\left(\operatorname{pr}_{2, V}^{\prime}\right) H_{*}(\tau) & =H_{*}\left(\operatorname{pr}_{2, V}\right) H_{*}\left(\mathrm{id}^{\prime}\right), \\
H^{*}\left(j_{f} \times \mathrm{id}_{g^{-1}(V)}\right) H^{*}\left(\operatorname{pr}_{1, U}^{\prime}\right) & =H^{*}\left(\operatorname{pr}_{1, V}\right) H^{*}\left(j_{f}\right),
\end{aligned}
$$

imply the claim (3.2.5).

For (2), it suffices to consider the case $U=S=\operatorname{Spec} R$. The ring homomorphisms $g^{*}: R \rightarrow H^{0}\left(X, \mathrm{O}_{X}\right)$ and $f^{*}: R \rightarrow H^{0}\left(Y, \mathscr{O}_{Y}\right)$ induce $R$-module structures on $H(X)$ and $H(Y)$, respectively.

We have to prove the following equality for all $r \in R$ and $a \in H^{i}\left(X, \Omega_{X}^{j}\right)$ :

$g^{*}(r) \cup H_{*}\left(\mathrm{pr}_{2}\right)\left(H^{*}\left(\mathrm{pr}_{1}\right)(a) \cup \operatorname{cl}([Z])\right)=H_{*}\left(\operatorname{pr}_{2}\right)\left(H^{*}\left(\operatorname{pr}_{1}\right)\left(f^{*}(r) \cup a\right) \cup \operatorname{cl}([Z])\right)$.

For this, it is enough to show that

$$
\begin{aligned}
& H^{*}\left(\operatorname{pr}_{2}\right)\left(g^{*}(r)\right) \cup \operatorname{cl}([Z]) \\
& \quad=H^{*}\left(\operatorname{pr}_{1}\right)\left(f^{*}(r)\right) \cup \operatorname{cl}([Z]) \text { in } H_{Z}^{d}\left(X \times Y, \Omega_{X \times Y}^{d}\right) .
\end{aligned}
$$

Choose an open set $U \subset X \times Y$ such that $Z \cap U$ is nonempty and smooth. Since the natural map $H_{Z}^{d}\left(X \times Y, \Omega_{X \times Y}^{d}\right) \rightarrow H_{Z \cap U}^{d}\left(U, \Omega_{U}^{d}\right)$ is injective, it suffices to check (3.2.6) on $H_{Z \cap U}^{d}\left(U, \Omega_{U}^{d}\right)$. We write $\iota_{1}: Z \cap U \rightarrow(U, Z \cap U)$ in $V_{*}$ and $\iota_{2}: Z \cap U \rightarrow U$ in $V^{*}$ for the obvious morphisms. By using the projection formula and $\operatorname{cl}([Z \cap U])=H_{*}\left(l_{1}\right)(1)$ we reduce to the statement

$$
H^{*}\left(l_{2}\right) H^{*}\left(\mathrm{pr}_{2}\right)\left(g^{*}(r)\right)=H^{*}\left(l_{2}\right) H^{*}\left(\mathrm{pr}_{1}\right)\left(f^{*}(r)\right) .
$$

This follows from $g \circ \mathrm{pr}_{2} \circ l_{2}=f \circ \mathrm{pr}_{1} \circ \iota_{2}$. 
Definition 3.2.7. Two integral schemes $X$ and $Y$ over a base $S$ are called properly birational over $S$ if there is an integral scheme $Z$ over $S$ and morphisms over $S$ :

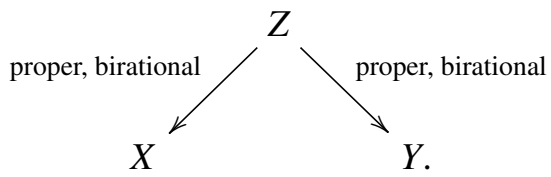

Theorem 3.2.8. Let $S$ be a scheme over a perfect field $k$. Let $f: X \rightarrow S$ and $g: Y \rightarrow S$ be integral $S$-schemes, that are smooth over $k$ and properly birational over $S$. Let $Z$ be an integral scheme together with proper birational morphisms $Z \rightarrow X$ and $Z \rightarrow Y$ such that

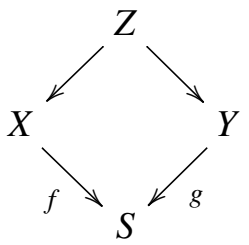

is commutative. We denote by $Z_{0}$ be the image of $Z$ in $X \times{ }_{S} Y$. Then, for all $i$, $\rho_{H}\left(Z_{0} / S\right)$ induces isomorphisms of $\mathcal{O}_{S}$-modules $(d=\operatorname{dim} X=\operatorname{dim} Y)$

$$
R^{i} f_{*} \mathrm{O}_{X} \stackrel{\simeq}{\rightarrow} R^{i} g_{*} \mathrm{O}_{Y} \text { and } R^{i} f_{*} \Omega_{X}^{d} \stackrel{\simeq}{\rightarrow} R^{i} g_{*} \Omega_{Y}^{d} \text {. }
$$

Proof. Recall that $\rho_{H}\left(Z_{0} / S\right)$ is defined in Proposition 3.2.4 as the sheafication of the maps

$$
\rho_{H} \circ \operatorname{cl}\left(\left[\left(Z_{0, U}\right]\right): H^{i}\left(f^{-1}(U), \Omega_{f^{-1}(U)}^{j}\right) \rightarrow H^{i}\left(g^{-1}(U), \Omega_{g^{-1}(U)}^{j}\right),\right.
$$

where $U$ runs over all open sets of $S$ and $Z_{0, U}$ denotes the restriction of $Z_{0}$ to $f^{-1}(U) \times{ }_{U} g^{-1}(U)$. By Proposition 3.2.4, $\rho_{H}\left(Z_{0} / S\right)$ is a morphism of $\mathcal{O}_{S^{-}}$ modules.

Obviously, it is sufficient to prove that (3.2.9), for $j=0$ and $j=d$, is an isomorphism for every open $U$. Thus we may suppose that $U=S, f^{-1}(U)=X$ and $g^{-1}(U)=Y, Z_{0, U}=Z$, and we need to prove that

$$
\begin{aligned}
& \rho_{H} \circ \operatorname{cl}\left(\left[\left(Z_{0}\right]\right): H^{i}\left(X, \odot_{X}\right) \rightarrow H^{i}\left(Y \odot_{Y}\right),\right. \\
& \rho_{H} \circ c l\left(\left[\left(Z_{0}\right]\right): H^{i}\left(X, \Omega_{X}^{d}\right) \rightarrow H^{i}\left(Y, \Omega_{Y}^{d}\right),\right.
\end{aligned}
$$

are isomorphisms for all $i$. In other words, we reduced to the case $S=\operatorname{Spec}(k)$.

Obviously, we may assume that $Z \subset X \times Y$. Let $Z^{\prime} \subset Z, X^{\prime} \subset X$ and $Y^{\prime} \subset Y$ be nonempty open subsets such that $\operatorname{pr}_{1}^{-1}\left(X^{\prime}\right)=Z^{\prime}$ and $\operatorname{pr}_{2}^{-1}\left(Y^{\prime}\right)=Z^{\prime}$, and such that $\mathrm{pr}_{1}: Z^{\prime} \rightarrow X^{\prime}$ and $\mathrm{pr}_{2}: Z^{\prime} \rightarrow Y^{\prime}$ are isomorphisms.

We obtain a correspondence $[Z] \in \operatorname{Hom}_{\mathrm{Cor}}(X, Y)^{0}$, and we denote by $\left[Z^{t}\right] \in$ $\operatorname{Hom}_{\mathrm{Cor}_{\mathrm{CH}}}(Y, X)^{0}$ the correspondence defined by $Z$ considered as subset of $Y \times X$. 
We claim that

$$
[Z] \circ\left[Z^{t}\right]=\Delta_{Y}+E_{1} \quad \text { and } \quad\left[Z^{t}\right] \circ[Z]=\Delta_{X}+E_{2},
$$

with cycles $E_{1}$ and $E_{2}$, supported in $\left(Y \backslash Y^{\prime}\right) \times\left(Y \backslash Y^{\prime}\right)$ and $\left(X \backslash X^{\prime}\right) \times\left(X \backslash X^{\prime}\right)$, respectively. Indeed, in view of Lemma 1.3.4, $\left[Z^{t}\right] \circ[Z]$ is naturally supported in

$\operatorname{supp}\left(Z, Z^{t}\right)=\left\{\left(x_{1}, x_{2}\right) \in X \times X \mid\left(x_{1}, y\right) \in Z,\left(y, x_{2}\right) \in Z^{t}\right.$ for some $\left.y \in Y\right\}$.

By using Lemma 1.3.6 for the open $X^{\prime} \subset X$, we conclude that $\left[Z^{t}\right] \circ[Z]$ maps to $\left[\Delta_{X^{\prime}}\right]$ via the localization map

$$
\mathrm{CH}\left(\operatorname{supp}\left(Z, Z^{t}\right)\right) \rightarrow \mathrm{CH}\left(\operatorname{supp}\left(Z, Z^{t}\right) \cap\left(X^{\prime} \times X^{\prime}\right)\right) .
$$

Thus

$$
\left[Z^{t}\right] \circ[Z]=\Delta_{X}+E_{2}
$$

with $E_{2}$ supported in $\operatorname{supp}\left(Z, Z^{t}\right) \backslash\left(X^{\prime} \times X^{\prime}\right)$. Finally, we observe that

$$
\operatorname{supp}\left(Z, Z^{t}\right) \cap\left(\left(X^{\prime} \times X\right) \cup\left(X \times X^{\prime}\right)\right)=\Delta_{X^{\prime}}=\operatorname{supp}\left(Z, Z^{t}\right) \cap\left(X^{\prime} \times X^{\prime}\right),
$$

and thus $E_{2}$ has support in $(X \times X) \backslash\left(\left(X^{\prime} \times X\right) \cup\left(X \times X^{\prime}\right)\right)=\left(X \backslash X^{\prime}\right) \times\left(X \backslash X^{\prime}\right)$. The same argument works for $[Z] \circ\left[Z^{t}\right]$.

Now, Proposition 3.2.2 implies that $\rho_{H} \circ \mathrm{cl}([Z])$ induce isomorphisms

$$
H^{*}\left(X, \mathcal{O}_{X}\right) \stackrel{\simeq}{\rightarrow} H^{*}\left(Y, \mathscr{O}_{Y}\right) \text { and } H^{*}\left(X, \Omega_{X}^{d}\right) \stackrel{\simeq}{\rightarrow} H^{*}\left(Y, \Omega_{Y}^{d}\right) \text {. }
$$

Corollary 3.2.10. Let $k$ be an arbitrary field and let $f: X \rightarrow Y$ be a proper birational morphism between smooth schemes $X$ and $Y$. Then

$$
R f_{*}\left(\mathrm{O}_{X}\right)=\mathrm{O}_{Y} \quad \text { and } \quad R f_{*}\left(\omega_{X}\right)=\omega_{Y} .
$$

Proof. By base change we may assume that $k$ is algebraically closed. The claim follows from Theorem 3.2.8 for $S=Y$ and $X=Z$.

3.2.11. Consider a commutative diagram

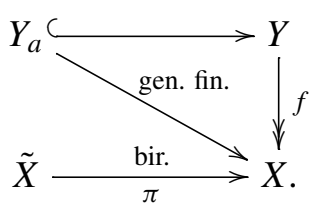

Here, all morphisms are proper and all schemes are integral, $\tilde{X}$ and $Y$ are smooth of dimension $d_{X}$ and $d_{Y}, \pi$ is birational, $f$ is surjective, $Y_{a} \rightarrow X$ is generically finite and surjective, and $Y_{a} \hookrightarrow Y$ is a closed immersion. Let $\eta$ be the generic point of $X$; then $Y_{a} \times_{X} \eta$ is finite over $\operatorname{Spec} k(\eta)$ of degree $\operatorname{deg}\left(Y_{a} / X\right)$. 
Choose a nonempty open set $U \subset X$ with $\pi: \pi^{-1}(U) \stackrel{\cong}{\rightarrow} U$ and such that $Y_{a}^{\prime}:=Y_{a} \cap f^{-1}(U) \subset f^{-1}(U) \stackrel{f}{\rightarrow} U$ is a finite morphism. Set

$$
Z_{a}=\overline{Y_{a}^{\prime} \times_{U} \pi^{-1}(U)} \subset Y_{a} \times_{X} \tilde{X},
$$

which gives a morphism $\left[Z_{a}\right]: Y \rightarrow \tilde{X}$ in $\operatorname{Cor}_{\mathrm{CH}}$. Furthermore, set

$$
\Gamma=\overline{\pi^{-1}(U) \times_{U} f^{-1}(U)} \subset \tilde{X} \times_{X} Y,
$$

which defines an element $[\Gamma]: \tilde{X} \rightarrow Y$ in $\mathrm{Cor}_{\mathrm{CH}}$. By using Lemmas 1.3.4 and 1.3.6, we obtain

$$
\left[Z_{a}\right] \circ[\Gamma]=\operatorname{deg}\left(Y_{a} / X\right) \cdot \operatorname{id}_{\tilde{X}}+E_{1},
$$

where $E_{1}$ has support in $\pi^{-1}(X \backslash U) \times \pi^{-1}(X \backslash U)$; thus $\rho_{H} \circ \mathrm{cl}\left(E_{1}\right)$ acts trivially on $H^{*}\left(\tilde{X}, \bigcirc_{\tilde{X}}\right) \oplus H^{*}\left(\tilde{X}, \Omega_{\tilde{X}}^{d_{X}}\right)$ by Proposition 3.2.2. On the other hand, Lemmas 1.3.4 and 1.3.6 imply

$$
[\Gamma] \circ\left[Z_{a}\right]=\overline{Y_{a}^{\prime} \times_{U} f^{-1}(U)}+E_{2},
$$

where $E_{2}$ has support in $f^{-1}(X \backslash U) \times f^{-1}(X \backslash U)$; thus $\rho_{H} \circ \operatorname{cl}\left(E_{2}\right)=0$ on $H^{*}\left(Y, \mathcal{O}_{Y}\right) \oplus H^{*}\left(Y, \Omega_{Y}^{d_{Y}}\right)$. Moreover, by using Lemmas 1.3.4 and 1.3.6 again,

$$
[\Gamma] \circ\left[Z_{a}\right] \circ[\Gamma] \circ\left[Z_{a}\right]=\operatorname{deg}\left(Y_{a} / X\right) \cdot \overline{Y_{a}^{\prime} \times_{U} f^{-1}(U)}+E_{3},
$$

with a cycle $E_{3}$ supported in $f^{-1}(X \backslash U) \times f^{-1}(X \backslash U)$, and therefore $\rho_{H} \circ \mathrm{cl}\left(E_{3}\right)=0$ on $H^{*}\left(Y, \mathscr{O}_{Y}\right) \oplus H^{*}\left(Y, \Omega_{Y}^{d_{Y}}\right)$.

We obtain an endomorphism

$$
P\left(Y_{a}\right):=\left.\rho_{H} \circ \operatorname{cl}\left([\Gamma] \circ\left[Z_{a}\right]\right)\right|_{H^{*}\left(Y, \mathscr{O}_{Y}\right) \oplus H^{*}\left(Y, \Omega_{Y}^{d_{Y}}\right)}
$$

of $H^{*}\left(Y, \mathscr{O}_{Y}\right) \oplus H^{*}\left(Y, \Omega_{Y}^{d_{Y}}\right)$ such that $P\left(Y_{a}\right)^{2}=\operatorname{deg}\left(Y_{a} / X\right) \cdot P\left(Y_{a}\right)$. Note that $P\left(Y_{a}\right)$ does not depend on $\tilde{X}$, because it is given by

$$
P\left(Y_{a}\right)=\rho_{H}\left(\operatorname{cl}\left(\left[\overline{Y_{a}^{\prime} \times f^{-1} U}\right]\right)\right) .
$$

Proposition 3.2.12. If $\operatorname{deg}\left(Y_{a} / X\right)$ is invertible in $k$ then

$$
\rho_{H} \circ \operatorname{cl}(\Gamma): H^{*}\left(\tilde{X}, \bigcirc_{\tilde{X}}\right) \oplus H^{*}\left(\tilde{X}, \Omega_{\tilde{X}}^{d_{X}}\right) \rightarrow \operatorname{image}\left(\left.P\left(Y_{a}\right)\right|_{H^{*}\left(Y, \mathscr{O}_{Y}\right) \oplus H^{*}\left(Y, \Omega_{Y}^{d_{Y}}\right)}\right)
$$

is a well-defined isomorphism.

Proof. Indeed

$$
\left.\left(\left(\rho_{H} \circ \operatorname{cl}\left(Z_{a}\right)\right) \circ\left(\rho_{H} \circ \operatorname{cl}(\Gamma)\right)\right)\right|_{H^{*}\left(\tilde{X}, \mathcal{O}_{\tilde{X}}\right) \oplus H^{*}\left(\tilde{X}, \Omega_{\tilde{X}}^{d_{X}}\right)}
$$

is multiplication by $\operatorname{deg}\left(Y_{a} / X\right)$. It follows that $\rho_{H} \circ \operatorname{cl}(\Gamma)$ is injective and the image is contained in the image of $P\left(Y_{a}\right)$. The opposite inclusion is obvious. 
Corollary 3.2.13. Let $Y, \tilde{X}$ and $X$ be as in Section 3.2.11. Let $a \in Y$ be a closed point of the generic fiber $f^{-1}(\eta)$ with $\operatorname{deg}_{k(\eta)}(a) \in k^{*}$; we denote the corresponding closed subvariety by $Y_{a}$. For $i \geq 0$, the following are equivalent:

(1) $R^{i} \pi_{*}\left(0_{\tilde{X}}\right) \oplus R^{i} \pi_{*}\left(\Omega_{\tilde{X}}^{d_{X}}\right)=0$.

(2) $P\left(Y_{a} \cap f^{-1}\left(X^{\prime}\right)\right)$ vanishes on

$$
H^{i}\left(f^{-1}\left(X^{\prime}\right), \bigcirc_{f^{-1}\left(X^{\prime}\right)}\right) \oplus H^{i}\left(f^{-1}\left(X^{\prime}\right), \Omega_{f^{-1}\left(X^{\prime}\right)}^{d_{X}}\right)
$$

for every affine open subset $X^{\prime} \subset X$.

Proof. In view of Proposition 3.2.12 we get

$$
H^{i}\left(\pi^{-1}\left(X^{\prime}\right), \bigcirc_{\pi^{-1}\left(X^{\prime}\right)}\right)=\operatorname{image}\left(\left.P\left(Y_{a} \cap f^{-1}\left(X^{\prime}\right)\right)\right|_{H^{i}\left(f^{-1}\left(X^{\prime}\right), \mathcal{O}_{f^{-1}\left(X^{\prime}\right)}\right)}\right)
$$

and

$$
H^{i}\left(\pi^{-1}\left(X^{\prime}\right), \Omega_{\pi^{-1}\left(X^{\prime}\right)}^{d_{X}}\right)=\operatorname{image}\left(\left.P\left(Y_{a} \cap f^{-1}\left(X^{\prime}\right)\right)\right|_{H^{i}\left(f^{-1}\left(X^{\prime}\right), \Omega_{f^{-1}\left(X^{\prime}\right)}^{d_{Y}}\right.}\right)
$$

for every open subset $X^{\prime} \subset X$.

Theorem 3.2.14. Let $k$ be an arbitrary field. Consider

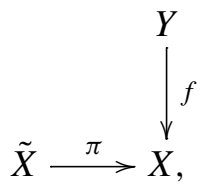

where $Y$ and $\tilde{X}$ are smooth and connected, $X$ is integral and normal, $f$ is surjective and finite with $\operatorname{deg}(f) \in k^{*}$, and finally $\pi$ is birational and proper. Then $X$ is Cohen-Macaulay and

$$
R \pi_{*} 0_{\tilde{X}}=\mathscr{O}_{X} \text { and } R \pi_{*} \omega_{\tilde{X}}=\omega_{X}
$$

where $\omega_{X}$ is the dualizing sheaf of $X$.

Proof. Choose an algebraic closure $\bar{k}$ of $k$. We claim that $X$ is geometrically normal, that is,

$$
X \times_{k} \bar{k}=\coprod_{i=1}^{r} X_{i} \quad \text { (disjoint union) }
$$

with $X_{i}$ integral and normal for all $i$. Indeed, since $X$ is normal we obtain

$$
\mathrm{O}_{X} \stackrel{=}{\rightarrow} \pi_{*} \mathrm{O}_{\tilde{X}}
$$


and this isomorphism is stable under the base change to $\bar{k}$. Because $\tilde{X}$ is smooth, $\tilde{X} \times{ }_{k} \bar{k}$ is a disjoint union of smooth schemes

$$
\tilde{X} \times_{k} \bar{k}=\coprod_{i=1}^{r} \tilde{X}_{i} .
$$

From $\mathrm{O}_{X \times{ }_{k} \bar{k}} \stackrel{=}{\rightarrow} \pi_{*} \mathrm{O}_{\tilde{X} \times_{k} \bar{k}}$ we conclude that $\pi \times_{k} \bar{k}$ has connected fibres; thus we obtain the equality $(3.2 .15)$ with $X_{i}:=\left(\pi \times_{k} \bar{k}\right)\left(\tilde{X}_{i}\right)$. Of course, $\tilde{X}_{i} \rightarrow X_{i}$ is birational, and $\mathrm{O}_{X_{i}} \stackrel{=}{\rightarrow}\left(\pi \times_{k} \bar{k}\right)_{*} \mathcal{O}_{\tilde{X}_{i}}$ implies that $X_{i}$ is normal.

We denote by $Y_{\bar{k}}, X_{\bar{k}}, \tilde{X}_{\bar{k}}$ the base change to $\bar{k}$, and by $\sigma_{X}: X_{\bar{k}} \rightarrow X$ the obvious morphism. Since

$$
\sigma_{X}^{*} R^{i} \pi_{*} 0_{\tilde{X}}=R^{i} \pi_{\bar{k} *} 0_{\tilde{X}_{\bar{k}}}, \quad \text { and } \quad \sigma_{X}^{*} R^{i} \pi_{*} \omega_{\tilde{X}}=R^{i} \pi_{\bar{k} *} \omega_{\tilde{X}_{\bar{k}}},
$$

and $\sigma_{X}$ is faithfully flat, it is sufficient to prove

$$
R^{i} \pi_{\bar{k} *} 0_{\tilde{X}_{\bar{k}}}=0=R^{i} \pi_{\bar{k} *} \omega_{\tilde{X}_{\bar{k}}} \text { for all } i>0 .
$$

Now $\tilde{X} \times{ }_{k} \bar{k}=\coprod_{i=1}^{r} \tilde{X}_{i}$ with $\tilde{X}_{i}$ smooth and connected such that $\left.\pi_{\bar{k}}\right|_{\tilde{X}_{i}}: \tilde{X}_{i} \rightarrow X_{i}$ is birational. We define $Y_{i}:=Y_{\bar{k}} \cap f_{\bar{k}}^{-1}\left(X_{i}\right)$ and let $Y_{i}=\bigsqcup_{j} Y_{i, j}$ be the decomposition into connected (smooth) components. Since $\operatorname{deg}\left(Y_{i} / X_{i}\right) \in k^{*}$ is invertible, there exists $j$ such that $\operatorname{deg}\left(Y_{i, j} / X_{i}\right) \in k^{*}$. Thus we are reduced to proving the claim for an algebraically closed field $k$.

Since $f$ is affine, the statement $R \pi_{*} \mathrm{O}_{\tilde{X}}=\mathrm{O}_{X}$ follows from Corollary 3.2.13 and $X$ normal. Applying $D_{X}$ and shifting by $\left[-d_{X}\right]$ (with $d_{X}=\operatorname{dim} X$ ), we obtain $R \pi_{*} \Omega_{\tilde{X}}^{d_{X}}=\pi_{X}^{!} k\left[-d_{X}\right]$ (with $\pi_{X}: X \rightarrow$ Spec $k$ the structure map). Now again by Corollary 3.2.13, we obtain $R^{i} \pi_{*} \Omega_{\tilde{X}}^{d_{X}}=0$ for all $i \neq 0$. Thus $\pi_{X}^{!} k\left[-d_{X}\right]=\omega_{X}$ is a sheaf and hence $X$ is Cohen-Macaulay.

\section{Generalization to tame quotients}

The goal of this section is to generalize Theorem 3.2.8 by replacing the assumption on the smoothness of $X$ and $Y$ with the weaker assumption that $X$ and $Y$ are tame quotients (see Definition 4.2.6). We already proved in Theorem 3.2.14 that the cohomology of the structure sheaf and the dualizing sheaf of a tame quotient behaves like for smooth schemes. Therefore it is a natural question to extend Theorem 3.2.8 in order to include tame quotients.

4.1. The action of finite correspondences. Let $X$ be a smooth scheme and $Z$ a closed integral subscheme of pure codimension $c$. Then $\mathcal{H}_{Z}^{j}\left(\Omega_{X}^{c}\right)=0$ for $j<c$. Consequently, there is a natural morphism

$$
\mathcal{H}_{Z}^{c}\left(\Omega_{X}^{c}\right)[-c] \rightarrow R \Gamma_{Z} \Omega_{X}^{c},
$$


which induces an isomorphism $H_{Z}^{c}\left(X, \Omega_{X}^{c}\right)=H^{0}\left(X, \mathscr{H}_{Z}^{c}\left(\Omega_{X}^{c}\right)\right)$.

Definition 4.1.2. Let $f: X \rightarrow Y$ be a morphism between smooth $k$-schemes of pure dimension $d_{X}$ and $d_{Y}$, respectively. Let $Z \subset X$ be a $c:=d_{X}-d_{Y}$ codimensional integral subscheme such that the restriction of $f$ to $Z$ is finite. Then we define for $q \geq 0$ the local push-forward

$$
f_{Z *}: f_{*} \mathscr{H}_{Z}^{c}\left(\Omega_{X}^{q}\right) \rightarrow \Omega_{Y}^{q-c}
$$

in the following way: Choose a compactification of $f$, that is, a proper morphism $\bar{f}: \bar{X} \rightarrow Y$ and an open immersion $j: X \hookrightarrow \bar{X}$ such that $f=\bar{f} \circ j$, and then define $f_{Z *}$ as the composition in $D_{\mathrm{qc}}^{+}(Y)$ of the natural map

$$
f_{*} \mathscr{H}_{Z}^{c}\left(\Omega_{X}^{q}\right) \stackrel{(4.1 .1)}{\longrightarrow} R f_{*}\left(R \underline{\Gamma}_{Z}\left(\Omega_{X}^{q}\right)[c]\right)
$$

with

$$
\begin{aligned}
R f_{*}\left(R \Gamma_{Z}\left(\Omega_{X}^{q}\right)[c]\right) & \stackrel{(2.2 .15)}{+ \text { excision }} R \bar{f}_{*} R \underline{\Gamma}_{Z}\left(D_{\bar{X}}\left(\Omega_{\bar{X}}^{d_{X}-q}\right)\left[c-d_{X}\right]\right) \\
& \stackrel{\text { forget support }}{\longrightarrow} R \bar{f}_{*} D_{\bar{X}}\left(\Omega_{\bar{X}}^{d_{X}-q}\right)\left[-d_{Y}\right] \\
& \stackrel{\bar{f}_{*}}{\longrightarrow} D_{Y}\left(\Omega_{Y}^{d_{X}-q}\right)\left[-d_{Y}\right] \stackrel{(2.2 .15)}{\longrightarrow} \Omega_{Y}^{q-c},
\end{aligned}
$$

where $\bar{f}_{*}$ is the morphism from Definition 2.2.5.

Applying $H^{0}(X, \cdot)$ gives a morphism

$$
H^{0}\left(Y, f_{*} \mathscr{H}_{Z}^{c}\left(\Omega_{X}^{q}\right)\right)=H^{0}\left(X, \mathscr{H}_{Z}^{c}\left(\Omega_{X}\right)\right)=H_{Z}^{c}\left(X, \Omega_{X}^{q}\right) \rightarrow H^{0}\left(Y, \Omega_{Y}^{q-c}\right),
$$

which by the definition coincides with the cohomological degree zero part of the pushforward for $f:(X, Z) \rightarrow Y$; see Definition 2.3.2. This also implies that $f_{Z *}$ is independent of the chosen compactification.

Definition 4.1.4. Let $S$ be a $k$-scheme and let $f: X \rightarrow S$ and $g: Y \rightarrow S$ be two integral $S$-schemes, which are smooth over $k$. Let $Z \subset X \times{ }_{S} Y$ be a closed integral subscheme such that $\operatorname{pr}_{2} \mid Z: Z \rightarrow Y$ is finite and surjective. In particular the codimension of $Z$ in $X \times Y$ equals $\operatorname{dim} X:=c$. The projections from $X \times Y$ to $X$ and $Y$ are denoted by $\operatorname{pr}_{1}$ and $\operatorname{pr}_{2}$, respectively. For all $q \geq 0$ we define a morphism

$$
\varphi_{Z}^{q}: f_{*} \Omega_{X}^{q} \rightarrow g_{*} \Omega_{Y}^{q}
$$

as follows: Let

$$
\operatorname{cl}(Z) \in H_{Z}^{c}\left(X \times Y, \Omega_{X \times Y}^{c}\right)=H^{0}\left(X \times Y, \mathscr{H}_{Z}^{c}\left(\Omega_{X \times Y}^{c}\right)\right)
$$

be the cycle class of $Z$. The cup product with $c l(Z)$ yields a morphism

$$
\cup \operatorname{cl}(Z): \Omega_{X \times Y}^{q} \rightarrow \mathcal{H}_{Z}^{c}\left(\Omega_{X \times Y}^{c+q}\right)
$$


and hence a morphism $f_{*} \operatorname{pr}_{1 *} \Omega_{X \times Y}^{q} \rightarrow f_{*} \operatorname{pr}_{1 *} \mathscr{H}_{Z}^{c}\left(\Omega_{X \times Y}^{c+q}\right)$. We claim that it also induces a morphism of $\mathrm{O}_{S}$-modules

$$
f_{*} \operatorname{pr}_{1 *} \Omega_{X \times Y}^{q} \rightarrow g_{*} \operatorname{pr}_{2 *} \mathcal{H}_{Z}^{c}\left(\Omega_{X \times Y}^{q+c}\right) .
$$

Indeed since $\mathscr{H}_{Z}^{c}\left(\Omega_{X \times Y}^{q+c}\right)$ has support in $Z \subset X \times{ }_{S} Y$, the two abelian sheaves

$$
g_{*} \operatorname{pr}_{2 *} \mathscr{H}_{Z}^{c}\left(\Omega_{X \times Y}^{q+c}\right) \text { and } f_{*} \operatorname{pr}_{1 *} \mathscr{H}_{Z}^{c}\left(\Omega_{X \times Y}^{q+c}\right)
$$

are equal; we denote this abelian sheaf on $S$ by $\mathscr{A}$. Now there are two $\mathcal{O}_{S}$-module structures on $\mathscr{A}$ : They are induced by

$$
\mathrm{O}_{S} \stackrel{g^{*}}{\rightarrow} g_{*} \mathrm{O}_{Y} \stackrel{\mathrm{pr}_{2}^{*}}{\rightarrow} g_{*} \mathrm{pr}_{2 *} \mathrm{O}_{X \times Y} \quad \text { and } \quad \mathrm{O}_{S} \stackrel{f^{*}}{\rightarrow} f_{*} \mathrm{O}_{X} \stackrel{\mathrm{pr}_{1}^{*}}{\rightarrow} f_{*} \mathrm{pr}_{1 *} \mathrm{O}_{X \times Y} .
$$

The claim (4.1.6) is now a consequence of the following equality in $\mathscr{A}$ :

$$
\operatorname{pr}_{2}^{*} g^{*}(a) \cdot(\beta \cup \operatorname{cl}(Z))=\operatorname{pr}_{1}^{*} f^{*}(a) \cdot(\beta \cup c l(Z))
$$

for all $a \in \mathcal{O}_{S}, \beta \in f_{*} \operatorname{pr}_{1 *} \Omega_{X \times c l(Z)) Y}^{q}$, which holds by (3.2.6). We can then define the morphism $\varphi_{Z}^{q}$ as the composition

$$
f_{*} \Omega_{X}^{q} \stackrel{\mathrm{pr}_{1}^{*}}{\longrightarrow} f_{*} \operatorname{pr}_{1 *} \Omega_{X \times Y}^{q} \stackrel{(4.1 .6)}{\longrightarrow} g_{*} \operatorname{pr}_{2 *} \mathscr{H}_{Z}^{c}\left(\Omega_{X \times Y}^{c+q}\right) \stackrel{\mathrm{pr}_{2, Z *}}{\longrightarrow} g_{*} \Omega_{Y}^{q} .
$$

We write $\varphi_{Z}=\bigoplus_{q} \varphi_{Z}^{q}$.

Let $\alpha=\sum_{i} n_{i}\left[Z_{i}\right]$ be a formal sum of integral closed subschemes $Z_{i}$ of $X \times{ }_{S} Y$, which are finite and surjective over $Y$, with coefficients $n_{i}$ in $\mathbb{Z}$. Then we define

$$
\varphi_{\alpha}:=\sum_{i} n_{i} \varphi_{Z_{i}}: \bigoplus_{q} f_{*} \Omega_{X}^{q} \rightarrow \bigoplus_{q} g_{*} \Omega_{Y}^{q} .
$$

Lemma 4.1.9. In the situation above, assume additionally that $g$ is affine. Then for any cycle $\alpha=\sum_{i} n_{i}\left[Z_{i}\right]$, with $Z_{i} \subset X \times{ }_{S} Y$ integral closed subschemes, which are finite and surjective over $Y$, and $n_{i} \in \mathbb{Z}$, we have the equality

$$
\oplus_{i} H^{i}\left(S, \varphi_{\alpha}\right)=\rho_{H}(c l(\bar{\alpha})): \bigoplus_{i, j} H^{i}\left(X, \Omega_{X}^{j}\right) \rightarrow \bigoplus_{i, j} H^{i}\left(Y, \Omega_{Y}^{j}\right),
$$

where $\bar{\alpha}$ is the image of $\alpha$ in $\mathrm{CH}_{\operatorname{dim} Y}\left(X \times Y, P\left(\Phi_{X}, \Phi_{Y}\right)\right)$ with $P\left(\Phi_{X}, \Phi_{X}\right)$ as in (1.3.8), $\rho_{H}$ is defined in 1.3.18 and cl is a shorthand notation for $\operatorname{Cor}(c l)$ with $\mathrm{cl}: \mathrm{CH} \rightarrow \mathrm{H}$ the morphism from 3.1.8.

Proof. Let $\pi: S \rightarrow$ Spec $k$ be the structure map. We may assume $\alpha=[Z]$ with $Z \subset X \times{ }_{S} Y$ an integral closed subscheme, which is finite and surjective over $Y$. It is 
easy to see that $\rho_{H}(\operatorname{cl}(\bar{\alpha}))$ is induced by taking the cohomology of the composition

$$
\begin{aligned}
R \pi_{*} f_{*} \Omega_{X}^{q} & \stackrel{\mathrm{pr}_{1}^{*}}{\longrightarrow} R \pi_{*} R\left(f \mathrm{pr}_{1}\right)_{*} \Omega_{X \times Y}^{q} \\
& \stackrel{\cup c l(Z)}{\longrightarrow} R \pi_{*} R\left(f \mathrm{pr}_{1}\right)_{*} \mathscr{H}_{Z}^{c}\left(\Omega_{X \times Y}^{q+c}\right) \\
& \stackrel{Z \subset X \times{ }_{S} Y}{\longrightarrow} R \pi_{*} R\left(g \mathrm{pr}_{2}\right)_{*} \mathscr{H}_{Z}^{c}\left(\Omega_{X \times Y}^{q+c}\right) \\
& \stackrel{\subseteq}{\longrightarrow} R \pi_{*}\left(g \mathrm{pr}_{2}\right)_{*} \mathscr{H}_{Z}^{c}\left(\Omega_{X \times Y}^{q+c}\right) \\
& \stackrel{(4.1 .1)}{\longrightarrow} R \pi_{*} R\left(g \mathrm{pr}_{2}\right)_{*} R \underline{\Gamma}\left(\Omega_{X \times Y}^{q+c}\right)[c] \\
& \stackrel{(4.1 .3)}{\longrightarrow} R \pi_{*} g_{*} \Omega_{Y}^{q} .
\end{aligned}
$$

We used for the fourth arrow the isomorphism

$$
\left(g \mathrm{pr}_{2}\right)_{*} \mathcal{H}_{Z}^{c}\left(\Omega_{X \times Y}^{q+c}\right) \stackrel{\simeq}{\rightarrow} R\left(g \mathrm{pr}_{2}\right)_{*} \mathscr{H}_{Z}^{c}\left(\Omega_{X \times Y}^{q+c}\right),
$$

because $\mathscr{H}_{Z}^{c}\left(\Omega_{X \times Y}^{q+c}\right)$ is a quasicoherent $\mathcal{O}_{X \times Y}$-module with support in $Z$, the map $Z \rightarrow Y$ is finite, and $g: Y \rightarrow S$ is affine. For the third arrow, notice that there is no map $R\left(f \mathrm{pr}_{1}\right)_{*} \mathscr{H}_{Z}^{c} \rightarrow R\left(g \mathrm{pr}_{2}\right)_{*} \mathscr{H}_{Z}^{c}$ in the derived category of $\mathrm{O}_{S}$-modules, but in the derived category of sheaves of $k$-vector spaces on $S$ these two complexes are isomorphic and that's all we need to define the third arrow.

We have to compare the morphism from (4.1.10) to (4.1.12) with $R \pi_{*} \varphi_{Z}$, where $\varphi_{Z}$ is defined in (4.1.7). Obviously, the morphism from (4.1.10) to (4.1.11) is equal to $R \pi_{*}\left((4.1 .6) \circ \mathrm{pr}_{1}^{*}\right)$. The morphism from (4.1.11) to (4.1.12) equals $R \pi_{*}\left(\mathrm{pr}_{2, Z *}\right)$, which proves the claim.

\subsection{Tame quotients.}

4.2.1. Let $X$ be a $k$-scheme that is normal, Cohen-Macaulay (CM) and equidimensional of pure dimension $n$ and denote by $\pi: X \rightarrow$ Spec $k$ its structure map.

Then $H^{i}\left(\pi^{!} k\right)=0$ for all $i \neq-n$; see [Conrad 2000, Theorem 3.5.1]. The dualizing sheaf of $X$ is then by definition

$$
\omega_{X}:=H^{-n}(\pi ! k) .
$$

We list some well-known properties:

(1) $\omega_{X}[n]$ is canonically isomorphic to $\pi^{!} k$ in $D_{c}^{+}(X)$.

(2) $\omega_{X}$ is a dualizing complex on $X$, that is, $\omega_{X}$ is coherent, has finite injective dimension and the natural map $\mathscr{O}_{X} \rightarrow R \mathscr{H o m}\left(\omega_{X}, \omega_{X}\right)$ is an isomorphism. (Indeed by [Hartshorne 1966, Chapter V, 10.1 and 10.2], $\pi^{!} k$ is a dualizing complex.)

(3) $\omega_{X}$ is $\mathrm{CM}$ with respect to the codimension filtration on $X$, that is,

$$
\operatorname{depth}_{\bigcirc_{X, x}} \omega_{X, x}=\operatorname{dim} \mathcal{O}_{X, x} \text { for all } x \in X,
$$


(By [Hartshorne 1966, Chapter V, Proposition 7.3] $\omega_{X}$ is Gorenstein, in particular CM, with respect to its associated filtration. Therefore we have to show that the associated codimension function to $\omega_{X}$ [Hartshorne 1966, V.7] is the usual codimension function. By [Hartshorne 1966, Chapter V, Proposition 7.1] it suffices to show that $\operatorname{Ext}_{\bigcirc_{X, \eta}}^{0}\left(k(\eta), \omega_{X, \eta}\right) \neq 0$ for all generic points $\eta \in X$. But $X$ is normal and thus $\omega_{X, \eta} \cong k(\eta)$.

(4) In case $X$ is smooth, $\omega_{X}$ is canonically isomorphic to $\Omega_{X}^{n}$, via the isomorphism

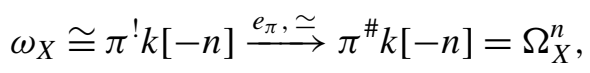

where $e_{\pi}: \pi^{!} \cong \pi^{\#}$ is the isomorphism from [Conrad 2000, (3.3.21)].

(5) If $u: U \rightarrow X$ is étale, then $u^{*} \omega_{X}$ is canonically isomorphic to $\omega_{U}$ via the isomorphism

$$
u^{*} \omega_{X}=u^{\#} \omega_{X} \stackrel{e_{u}, \simeq}{\longrightarrow} u^{!} \omega_{X} \cong u^{!} \pi ! k \stackrel{c_{u, \pi}^{-1}, \simeq}{\longrightarrow}(\pi \circ u)^{!} k \cong \omega_{U},
$$

where $c_{u, \pi}:(\pi \circ u)^{!} \cong u^{!} \circ \pi^{!}$is the isomorphism from [Conrad 2000, 3.3.14].

(6) Let $U$ be an open subscheme of $X$ that is smooth over $k$ and contains all 1-codimensional points and denote by $j: U \hookrightarrow X$ the corresponding open immersion. Then adjunction induces an isomorphism

$$
\omega_{X} \stackrel{\simeq}{\rightarrow} j_{*} j^{*} \omega_{X} \cong j_{*} \omega_{U} \cong j_{*} \Omega_{U}^{n},
$$

where the last two isomorphisms are induced by (4) and (5). (This follows from (3). Indeed, let $V \subset X$ be open; then $\Gamma\left(V, \omega_{X}\right) \rightarrow \Gamma\left(V, j_{*} j^{*} \omega_{X}\right)=$ $\Gamma\left(V \cap U, \omega_{X}\right)$ is the restriction. Since all points in the complement of $U$ have codimension $\geq 2$, we obtain from (3) that $\operatorname{depth}\left(\omega_{X, x}\right) \geq 2$ for all $x \in X \backslash U$. Therefore $\Gamma\left(V, \omega_{X}\right) \rightarrow \Gamma\left(V \cap U, \omega_{X}\right)$ is bijective by [Grothendieck 1968, Exposé III, Corollary 3.5].)

4.2.2. Let $X$ be smooth and $Y$ a normal CM scheme both of pure dimension $n$, and let $f: X \rightarrow Y$ be a finite and surjective morphism. Then we have the usual pull-back on the structure sheaves $f^{*}: \mathscr{O}_{Y} \rightarrow f_{*} \mathscr{O}_{X}$ as well as a trace map $\tau_{f}^{0}: f_{*} \mathrm{O}_{X} \rightarrow \mathscr{O}_{Y}$, which extends the usual trace over the smooth locus of $Y$ (over which $f$ is flat). We define a pull-back and a trace between the dualizing sheaves as follows.

Definition 4.2.3. Let $X$ be smooth and let $Y$ be a normal CM scheme, both of pure dimension $n$, and let $f: X \rightarrow Y$ be a finite and surjective morphism.

(1) We define a pullback morphism

$$
f^{*}: \omega_{Y} \rightarrow f_{*} \omega_{X}
$$


as follows: Choose $j: U \hookrightarrow Y$ open and smooth over $k$ such that it contains all 1-codimensional points of $Y$; let $j^{\prime}: U^{\prime}=X \times_{Y} U \rightarrow X$ and $f^{\prime}: U^{\prime} \rightarrow U$ be the base changes of $j$ and $f$. Then we define $f^{*}$ as the composition

$$
\omega_{Y} \simeq j_{*} \Omega_{U}^{n} \stackrel{f^{\prime *}}{\longrightarrow} j_{*} f_{*}^{\prime} \Omega_{U^{\prime}}^{n}=f_{*} j_{*}^{\prime} \omega_{U^{\prime}} \cong f_{*} \omega_{X}
$$

for the last isomorphism observe that $U^{\prime}$ contains all 1-codimensional points of $X$. It is straightforward to check that this morphism is independent of the choice of $U$. (One only needs the compatibility statements (VAR1) and (VAR3) of [Hartshorne 1966, Chapter VII, Corollary 3.4(a)].)

(2) We define the trace

$$
\tau_{f}^{n}: f_{*} \omega_{X} \rightarrow \omega_{Y}
$$

as the composition in $D_{c}^{+}(Y)$

$$
f_{*} \omega_{X} \cong f_{*} \pi_{X}^{!} k[-n] \stackrel{c_{f, \pi_{Y}}}{\longrightarrow} f_{*} f^{!} \pi_{Y}^{!} k[-n] \stackrel{\operatorname{Tr}_{f}}{\longrightarrow} \pi_{Y}^{!} k[-n] \cong \omega_{Y},
$$

where $\pi_{X}$ and $\pi_{Y}$ are the structure maps of $X$ and $Y$ and $\operatorname{Tr}_{f}$ is the trace morphism [Conrad 2000, (3.3.2)].

We write $f^{*}: \mathscr{O}_{Y} \oplus \omega_{Y} \rightarrow f_{*}\left(\mathscr{O}_{X} \oplus \omega_{X}\right)$ for the sum of the usual pull-back with the pull-back defined in (1), and write $\tau_{f}:=\tau_{f}^{0} \oplus \tau_{f}^{n}: f_{*}\left(\mathcal{O}_{X} \oplus \omega_{X}\right) \rightarrow \mathbb{O}_{Y} \oplus \omega_{Y}$.

Remark 4.2.4. By its definition, the $\tau_{f}$ constructed above equals, when restricted to the smooth locus of $Y$, the $\tau_{f}$ from Proposition 2.2.23.

Corollary 4.2.5. Let $X, Y$ and $f$ be as in Definition 4.2.3. Suppose that $X$ is connected. Then the composition

$$
\tau_{f} \circ f^{*}: \mathcal{O}_{Y} \oplus \omega_{Y} \rightarrow \mathrm{O}_{Y} \oplus \omega_{Y}
$$

is equal to multiplication with the degree of $f$.

Proof. We have to check that the section $s=\tau_{f} \circ f^{*}-\operatorname{deg} f$ of

$$
H^{0}\left(Y, \operatorname{Hom}_{Y}\left(\mathscr{O}_{Y}, \mathscr{O}_{Y}\right)\right) \oplus H^{0}\left(Y, \operatorname{Hom}_{Y}\left(\omega_{Y}, \omega_{Y}\right)\right)
$$

is zero. But $H^{0}\left(Y, \mathscr{H}_{\operatorname{om}_{Y}}\left(\mathscr{O}_{Y}, \mathscr{O}_{Y}\right)\right)=H^{0}\left(Y, \mathscr{O}_{Y}\right)=H^{0}\left(Y, \mathscr{H o m}_{Y}\left(\omega_{Y}, \omega_{Y}\right)\right.$ ) (for the last equality we need that $\omega_{Y}$ is a dualizing complex). Therefore it is enough to check that $s$ is zero over an open and dense subset $U$ of $Y$. We may choose $U$ such that it is smooth and contains all 1-codimensional points of $Y$. Thus the statement follows from Proposition 2.2.23(3).

Definition 4.2.6. Let $X$ be a $k$-scheme. We say that $X$ is a tame quotient if $X$ is integral and normal and there exists a smooth and integral scheme $X^{\prime}$ with a finite and surjective morphism $f: X^{\prime} \rightarrow X$ whose degree is invertible in $k$. 
Remark 4.2.7. Assume $X$ is a tame quotient. Then $X$ is CM; see [Kollár and Mori 1998, Proposition 5.7(1)].

We may describe the cohomology of the structure sheaf and of the dualizing sheaf of a tame quotient as a direct summand of the corresponding cohomology of a smooth scheme as follows.

Proposition 4.2.8. Let $f: X \rightarrow Y$ be a finite and surjective morphism between integral schemes. Assume $X$ is smooth and $Y$ is normal. Furthermore, we assume that $\operatorname{deg} f$ is invertible in $k$. Set

$$
\alpha:=\left[X \times_{Y} X\right] \text { in } \mathrm{CH}_{\mathrm{dim} X}\left(X \times X, P\left(\Phi_{X}, \Phi_{X}\right)\right)=: \operatorname{Hom}_{\mathrm{Cor}_{\mathrm{CH}}}(X, X)^{0},
$$

(see (1.3.8) for the definition of $\left.P\left(\Phi_{X}, \Phi_{X}\right)\right)$. Then, for all $i$, the pull-back morphism

$$
f^{*}:\left(H^{i}\left(Y, \mathscr{O}_{Y}\right) \oplus H^{i}\left(Y, \omega_{Y}\right)\right) \rightarrow\left(H^{i}\left(X, \mathscr{O}_{X}\right) \oplus H^{i}\left(X, \omega_{X}\right)\right)
$$

induces an isomorphism

$$
\left(H^{i}\left(Y, \mathscr{O}_{Y}\right) \oplus H^{i}\left(Y, \omega_{Y}\right)\right) \cong \rho_{H}(c l(\alpha))\left(H^{i}\left(X, \mathscr{O}_{X}\right) \oplus H^{i}\left(X, \omega_{X}\right)\right) .
$$

(The functor $\rho_{H}$ is defined in 1.3.18 and cl is a shorthand notation for $\operatorname{Cor}(\mathrm{cl})$ with $\mathrm{cl}: \mathrm{CH} \rightarrow \mathrm{H}$ the morphism from Theorem 3.1.8.)

Proof. Write $\alpha=\left[X \times_{Y} X\right]=\sum_{T} n_{T}[T]$, where the sum is over all irreducible components $T$ of $X \times_{Y} X$. Notice that all the $T$ have dimension equal to $\operatorname{dim} X$ and project (via both projections) finitely and surjectively to $X$. Therefore

$$
\varphi_{\alpha}: f_{*}\left(\mathcal{O}_{X} \oplus \omega_{X}\right) \rightarrow f_{*}\left(\mathcal{O}_{X} \oplus \omega_{X}\right)
$$

is defined, where $\varphi_{\alpha}$ is the morphism from Definition 4.1.4. By Lemma 4.1.9 we have, for all $i$,

$$
H^{i}\left(Y, \varphi_{\alpha}\right)=\rho_{H} \circ \operatorname{cl}(\alpha): H^{i}\left(X, \mathcal{O}_{X} \oplus \omega_{X}\right) \rightarrow H^{i}\left(X, \mathcal{O}_{X} \oplus \omega_{X}\right) .
$$

We claim

$$
\varphi_{\alpha}=f^{*} \circ \tau_{f}: f_{*}\left(\mathcal{O}_{X} \oplus \omega_{X}\right) \rightarrow f_{*}\left(\mathcal{O}_{X} \oplus \omega_{X}\right) .
$$

Let $U \subset Y$ be a nonempty smooth open subscheme that contains all 1-codimensional points of $Y$. Then $f^{-1}(U)$ is smooth and contains all 1-codimensional points of $X$. Hence for any open $V \subset Y$, the restriction map

$$
H^{0}\left(f^{-1}(V), \mathscr{O}_{X} \oplus \omega_{X}\right) \rightarrow H^{0}\left(f^{-1}(V) \cap f^{-1}(U), \mathscr{O}_{X} \oplus \omega_{X}\right)
$$

is an isomorphism; see [Grothendieck 1968, Exposé III, Corollary 3.5]. Since both maps in (4.2.10) are compatible with restriction to open subsets of $Y$, we may therefore assume that $Y$ is smooth. In particular $f$ is flat and thus $\alpha$ equals $\left[\Gamma_{f}^{t}\right] \circ\left[\Gamma_{f}\right]$, where $\Gamma_{f}$ is the graph of $f$ and $\Gamma_{f}^{t}$ its transposed. Now the identity 
(4.2.10) follows from (4.2.9) (in the case $i=0$ ), Proposition 2.3.3(3) and 3.2.1. Thus applying again (4.2.9), we obtain

$$
\begin{aligned}
\rho_{H}(\operatorname{cl}(\alpha))\left(H^{i}\left(X, \mathscr{O}_{X}\right)\right. & \left.\oplus H^{i}\left(X, \omega_{X}\right)\right) \\
& =\operatorname{Image}\left(f^{*} \circ \tau_{f}: H^{i}\left(X, \mathcal{O}_{X} \oplus \omega_{X}\right)\right) \rightarrow H^{i}\left(X, \mathcal{O}_{X} \oplus \omega_{X}\right) .
\end{aligned}
$$

Since $\tau_{f} \circ f^{*}:\left(H^{i}\left(Y, \mathscr{O}_{Y}\right) \oplus H^{i}\left(Y, \omega_{Y}\right)\right) \rightarrow\left(H^{i}\left(Y, \mathscr{O}_{Y}\right) \oplus H^{i}\left(Y, \omega_{Y}\right)\right)$ is multiplication with the degree of $f$ (by Corollary 4.2.5) the proposition follows.

\subsection{Main theorem for tame quotients.}

Theorem 4.3.1. Let $S$ be a scheme over a perfect field $k$. Let $\pi_{X}: X \rightarrow S$ and $\pi_{Y}$ : $Y \rightarrow S$ be two integral $S$-schemes, which are tame quotients (see Definition 4.2.6). Furthermore, we assume that $X$ and $Y$ are properly birational equivalent. Then any $Z$ as in Definition 3.2.7 induces isomorphisms of $\mathcal{O}_{S}$-modules

$$
R^{i} \pi_{X *} \mathcal{O}_{X} \cong R^{i} \pi_{Y *} \mathcal{O}_{Y} \quad \text { and } \quad R^{i} \pi_{X *} \omega_{X} \cong R^{i} \pi_{Y *} \omega_{Y} \quad \text { for all } i \geq 0 .
$$

These isomorphisms depend only on the $\mathscr{O}_{S, \eta}$-isomorphism $k(X) \cong k(Y)$ induced by $Z$, where $\eta=\pi_{X}$ (generic point of $\left.X\right)=\pi_{Y}$ (generic point of $\left.Y\right)$.

Proof.

Claim 1. There are isomorphisms as in the statement in the case $S=\operatorname{Spec} k$.

Choose integral and smooth schemes $X^{\prime}$ and $Y^{\prime}$ with finite and surjective morphisms $f: X^{\prime} \rightarrow X$ and $g: Y^{\prime} \rightarrow Y$ whose degree is invertible in $k$. Choose $Z$ as in Definition 3.2.7. We may assume that $Z \subset X \times Y$ is a closed integral subscheme. We define $Z_{X^{\prime}}, Z_{Y^{\prime}}$ and $Z^{\prime}$ by the cartesian diagram

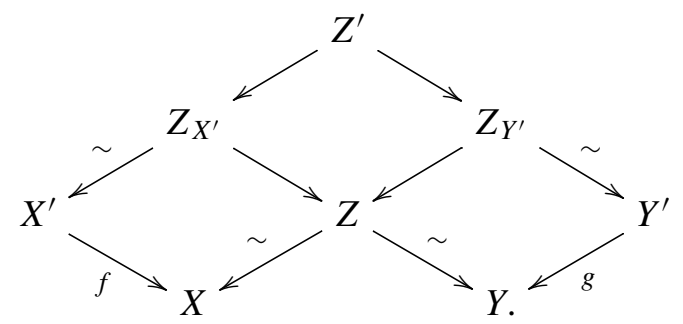

Here the arrows with an $\sim$ are proper and birational morphisms between integral schemes, and all other morphisms are finite and surjective. We may identify $Z^{\prime}$ with a closed subscheme of $X^{\prime} \times Y^{\prime}$ whose irreducible components are proper and surjective over both $X^{\prime}$ and $Y^{\prime}$, and all irreducible components have the same dimension equal to $d:=\operatorname{dim} X=\operatorname{dim} X^{\prime}=\operatorname{dim} Y=\operatorname{dim} Y^{\prime}$ (since $f$ and $g$ are finite and universally equidimensional). Therefore $Z^{\prime}$ and its transpose define cycles $\left[Z^{\prime}\right] \in \mathrm{CH}^{d}\left(X^{\prime} \times Y^{\prime}, P\left(\Phi_{X^{\prime}}, \Phi_{Y^{\prime}}\right)\right) \quad$ and $\quad\left[Z^{\prime}\right]^{t} \in \mathrm{CH}^{d}\left(Y^{\prime} \times X^{\prime}, P\left(\Phi_{Y^{\prime}}, \Phi_{X^{\prime}}\right)\right)$. 
Now choose nonempty smooth open subschemes $X_{o}, Y_{o}$ of $X, Y$ such that the morphisms $Z \rightarrow X, Z \rightarrow Y$ induce isomorphisms $Z_{o} \stackrel{\simeq}{\rightarrow} X_{o}, Z_{o} \stackrel{\simeq}{\rightarrow} Y_{o}$ with

$$
Z_{o}:=X_{o} \times_{X} Z=Z \times_{Y} Y_{o}
$$

Set $X_{o}^{\prime}=f^{-1}\left(X_{o}\right)$ and $Y_{o}^{\prime}=g^{-1}\left(Y_{o}\right)$ and denote by $f_{o}$ and $g_{o}$ the restrictions of $f$ and $g$ to $X_{o}^{\prime}$ and $Y_{o}^{\prime}$, respectively. We define $Z_{X_{o}^{\prime}}, Z_{Y_{o}^{\prime}}$ and $Z_{o}^{\prime}$ by the cartesian diagram

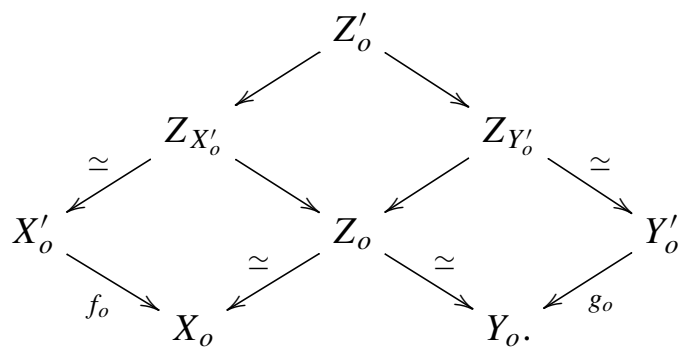

Here the arrows with an $\simeq$ are isomorphisms, and all other arrows are finite and surjective. We set $X_{c}^{\prime}=X^{\prime} \backslash X_{o}^{\prime}$ and $Y_{c}^{\prime}=Y^{\prime} \backslash Y_{o}^{\prime}$; these are closed subsets of codimension $\geq 1$. Now we define

$$
\begin{aligned}
\alpha & :=\left[X^{\prime} \times_{X} X^{\prime}\right] \in \mathrm{CH}^{d}\left(X^{\prime} \times X^{\prime}, P\left(\Phi_{X^{\prime}}, \Phi_{X^{\prime}}\right)\right), \\
\beta & :=\left[Y^{\prime} \times_{Y} Y^{\prime}\right] \in \mathrm{CH}^{d}\left(Y^{\prime} \times Y^{\prime}, P\left(\Phi_{Y^{\prime}}, \Phi_{Y^{\prime}}\right)\right) .
\end{aligned}
$$

We claim

$$
\begin{gathered}
\operatorname{deg} g \cdot\left(\left[Z^{\prime}\right] \circ \alpha\right)-\operatorname{deg} f \cdot\left(\beta \circ\left[Z^{\prime}\right]\right) \in \operatorname{image}\left(\mathrm{CH}_{*}\left(X_{c}^{\prime} \times Y_{c}^{\prime}\right)\right), \\
\operatorname{deg} f \cdot\left(\left[Z^{\prime}\right]^{t} \circ \beta\right)-\operatorname{deg} g \cdot\left(\alpha \circ\left[Z^{\prime}\right]^{t}\right) \in \operatorname{image}\left(\mathrm{CH}_{*}\left(Y_{c}^{\prime} \times X_{c}^{\prime}\right)\right), \\
\left(\left[Z^{\prime}\right]^{t} \circ\left[Z^{\prime}\right] \circ \alpha\right)-\operatorname{deg} f \operatorname{deg} g \cdot \alpha \in \operatorname{image}\left(\mathrm{CH}_{*}\left(X_{c}^{\prime} \times X_{c}^{\prime}\right)\right), \\
\left(\left[Z^{\prime}\right] \circ\left[Z^{\prime}\right]^{t} \circ \beta\right)-\operatorname{deg} f \operatorname{deg} g \cdot \beta \in \operatorname{image}\left(\mathrm{CH}_{*}\left(Y_{c}^{\prime} \times Y_{c}^{\prime}\right)\right) .
\end{gathered}
$$

By symmetry, it suffices to prove (4.3.2) and (4.3.4). Let us prove (4.3.2). By using Lemma 1.3.4 we can consider

$$
\alpha \in \mathrm{CH}\left(X^{\prime} \times_{X} X^{\prime}\right), \quad \beta \in \mathrm{CH}\left(Y^{\prime} \times_{Y} Y^{\prime}\right), \quad\left[Z^{\prime}\right] \in \mathrm{CH}\left(Z^{\prime}\right),
$$

and see that $\left[Z^{\prime}\right] \circ \alpha$ and $\beta \circ\left[Z^{\prime}\right]$ are naturally supported in $\mathrm{CH}\left(Z^{\prime}\right)$.

Since $Z^{\prime} \cap\left(\left(X_{o}^{\prime} \times Y^{\prime}\right) \cup\left(X^{\prime} \times Y_{o}^{\prime}\right)\right)=Z^{\prime} \cap\left(X_{o}^{\prime} \times Y_{o}^{\prime}\right)$, Lemma 1.3.6 and the localization sequence for Chow groups implies the claim if the equality

$$
\left.\left.\operatorname{deg}(g) \cdot\left[Z^{\prime}\right]\right|_{X^{\prime} \times Y_{o}^{\prime}} \circ \alpha\right|_{X_{o}^{\prime} \times X^{\prime}}=\left.\left.\operatorname{deg}(f) \cdot \beta\right|_{Y^{\prime} \times Y_{o}^{\prime}} \circ\left[Z^{\prime}\right]\right|_{X_{o}^{\prime} \times Y^{\prime}}
$$


holds in $\mathrm{CH}\left(Z^{\prime} \cap\left(X_{o}^{\prime} \times Y_{o}^{\prime}\right)\right)$. Here we have

$$
\begin{aligned}
\left.\alpha\right|_{X_{o}^{\prime} \times X^{\prime}} & \in \mathrm{CH}\left(X_{o}^{\prime} \times{ }_{X} X^{\prime}\right)=\mathrm{CH}\left(X_{o}^{\prime} \times_{X_{o}} X_{o}^{\prime}\right), \\
\left.\beta\right|_{Y^{\prime} \times Y_{o}^{\prime}} & \in \mathrm{CH}\left(Y^{\prime} \times{ }_{Y} Y_{o}^{\prime}\right)=\mathrm{CH}\left(Y_{o}^{\prime} \times_{Y_{o}} Y_{o}^{\prime}\right), \\
{\left.\left[Z^{\prime}\right]\right|_{X_{o}^{\prime} \times Y^{\prime}} } & \in \mathrm{CH}\left(Z^{\prime} \cap\left(X_{o}^{\prime} \times Y^{\prime}\right)\right)=\mathrm{CH}\left(Z^{\prime} \cap\left(X_{o}^{\prime} \times Y_{o}^{\prime}\right)\right), \\
{\left.\left[Z^{\prime}\right]\right|_{X^{\prime} \times Y_{o}^{\prime}} } & \in \mathrm{CH}\left(Z^{\prime} \cap\left(X^{\prime} \times Y_{o}^{\prime}\right)\right)=\mathrm{CH}\left(Z^{\prime} \cap\left(X_{o}^{\prime} \times Y_{o}^{\prime}\right)\right) .
\end{aligned}
$$

Obviously,

$$
\begin{aligned}
\left.\alpha\right|_{X_{o}^{\prime} \times X^{\prime}} & =\left[X_{o}^{\prime} \times X_{o} X_{o}^{\prime}\right]=\left[\Gamma_{f_{o}}^{t}\right] \circ\left[\Gamma_{f_{o}}\right], \\
\left.\beta\right|_{Y^{\prime} \times Y_{o}^{\prime}} & =\left[Y_{o}^{\prime} \times Y_{o} Y_{o}^{\prime}\right]=\left[\Gamma_{g_{o}}^{t}\right] \circ\left[\Gamma_{g_{o}}\right], \\
{\left.\left[Z^{\prime}\right]\right|_{X_{o}^{\prime} \times Y^{\prime}} } & =\left[Z_{o}^{\prime}\right]=\left[\Gamma_{g_{o}}^{t}\right] \circ\left[Z_{o}\right] \circ\left[\Gamma_{f_{o}}\right], \\
{\left.\left[Z^{\prime}\right]\right|_{X^{\prime} \times Y_{o}^{\prime}} } & =\left[Z_{o}^{\prime}\right] .
\end{aligned}
$$

Thus (4.3.6) follows from

$$
\begin{aligned}
& {\left[\Gamma_{f_{o}}\right] \circ\left[\Gamma_{f_{o}}^{t}\right]=\operatorname{deg}(f)\left[\Delta_{X_{o}}\right],} \\
& {\left[\Gamma_{g_{o}}\right] \circ\left[\Gamma_{g_{o}}^{t}\right]=\operatorname{deg}(g)\left[\Delta_{Y_{o}}\right] .}
\end{aligned}
$$

This finishes the proof of (4.3.2). The proof of (4.3.4) is similar. The cycles $\left[Z^{\prime}\right]^{t} \circ\left[Z^{\prime}\right] \circ \alpha$ and $\alpha$ are supported in

$$
B=\left\{\left(x_{1}^{\prime}, x_{2}^{\prime}\right) \in X^{\prime} \times X^{\prime} \mid\left(f\left(x_{1}^{\prime}\right), y\right) \in Z,\left(f\left(x_{2}^{\prime}\right), y\right) \in Z \text { for some } y \in Y\right\} .
$$

We see that $B \cap\left(\left(X_{o}^{\prime} \times X^{\prime}\right) \cup\left(X^{\prime} \times X_{o}^{\prime}\right)\right)=B \cap\left(X_{o}^{\prime} \times X_{o}^{\prime}\right)$, and by using Lemma 1.3.6 it is sufficient to prove

$$
\left[Z_{o}^{\prime}\right]^{t} \circ\left[Z_{o}^{\prime}\right] \circ\left[X_{o}^{\prime} \times_{X_{o}} X_{o}^{\prime}\right]=\operatorname{deg} f \operatorname{deg} g \cdot\left[X_{o}^{\prime} \times_{X_{o}} X_{o}^{\prime}\right] .
$$

In view of (4.3.7), this follows immediately from (4.3.8).

Since $\operatorname{deg} f$ and $\operatorname{deg} g$ are invertible in $k$, it follows from Proposition 3.2.2 and (4.3.2) that $(\operatorname{deg} f)^{-1} \rho_{H} \circ \operatorname{cl}\left(\left[Z^{\prime}\right]\right)$ induces a morphism

$$
\left(\rho_{H} \circ \operatorname{cl}(\alpha)\right) H^{*}\left(X^{\prime}, \mathscr{O}_{X^{\prime}} \oplus \omega_{X^{\prime}}\right) \rightarrow\left(\rho_{H} \circ \operatorname{cl}(\beta)\right) H^{*}\left(Y^{\prime}, \mathscr{O}_{Y^{\prime}} \oplus \omega_{Y^{\prime}}\right)
$$

and by (4.3.3), $(\operatorname{deg} g)^{-1} \rho_{H} \circ \operatorname{cl}\left(\left[Z^{\prime}\right]^{t}\right)$ induces a morphism

$$
\left(\rho_{H} \circ \operatorname{cl}(\beta)\right) H^{*}\left(Y^{\prime}, \mathcal{O}_{Y^{\prime}} \oplus \omega_{Y^{\prime}}\right) \rightarrow\left(\rho_{H} \circ \operatorname{cl}(\alpha)\right) H^{*}\left(X^{\prime}, \mathcal{O}_{X^{\prime}} \oplus \omega_{X^{\prime}}\right) .
$$

By (4.3.4) and (4.3.5) these two morphisms mutually inverse. So Proposition 4.2.8 yields isomorphisms

$$
H^{i}\left(X, \mathscr{O}_{X}\right) \cong H^{i}\left(Y, \mathscr{O}_{Y}\right) \text { and } \quad H^{i}\left(X, \omega_{X}\right) \cong H^{i}\left(Y, \omega_{Y}\right) \text { for all } i \geq 0 .
$$

This proves Claim 1. 
Claim 2. The isomorphisms constructed in Claim 1 depends only on the isomorphism $k(X) \cong k(Y)$ induced by a $Z$.

We use the shorthand notation $H^{i}(X)=H^{i}\left(X, \mathscr{O}_{X} \oplus \omega_{X}\right)$. Choose $Z$ as in Definition 3.2.7. Denote by $Z_{0}$ the image of $Z$ in $X \times Y$. Choose $f_{1}: X_{1} \rightarrow X$ and $g_{1}: Y_{1} \rightarrow Y$ finite and surjective, with $X_{1}, Y_{1}$ smooth and integral and deg $f_{1}$, $\operatorname{deg} g_{1} \in k^{*}$. Define

$$
\begin{aligned}
\underline{\alpha}_{1} & :=\rho_{H} \circ \operatorname{cl}\left(\left[X_{1} \times_{X} X_{1}\right]\right), \quad \underline{\beta}_{1}:=\rho_{H} \circ \operatorname{cl}\left(\left[Y_{1} \times_{Y} Y_{1}\right]\right), \\
\underline{\gamma}_{1}(Z) & :=\left(\operatorname{deg} f_{1}\right)^{-1} \rho_{H} \circ c l\left(\left[X_{1} \times_{X} Z_{0} \times_{Y} Y_{1}\right]\right) .
\end{aligned}
$$

Then, as seen in the proof of Claim 1 above, we obtain isomorphisms

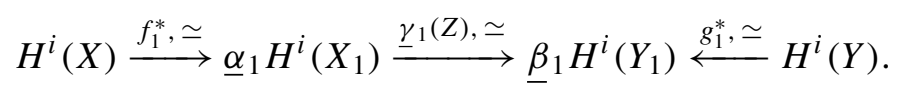

Now choose two different $Z$ as in Definition 3.2.7, say $Z_{1}$ and $Z_{2}$, which induce the same isomorphism $k(X) \cong k(Y)$. Then we can find smooth open subschemes $X_{o}, Y_{o}, Z_{1, o}, Z_{2, o}$ of $X, Y, Z_{1}, Z_{2}$ such that for $i=1$, 2, we have

$$
Z_{i, o}=Z_{i} \times_{X} X_{o}=Z_{i} \times_{Y} Y_{o}
$$

the projections $Z_{i, o} \stackrel{\simeq}{\rightarrow} X_{o}, Z_{i, o} \stackrel{\simeq}{\rightarrow} Y_{o}$ are isomorphisms, and the induced isomorphisms $h_{i}: X_{o} \stackrel{\simeq}{\rightarrow} Z_{o, i} \stackrel{\simeq}{\rightarrow} Y_{o}$ for $i=1,2$ are equal. Proposition 3.2.2 implies $\underline{\gamma}_{1}\left(Z_{1}\right)=\underline{\gamma}_{1}\left(Z_{2}\right)$ on $H^{i}\left(X_{1}\right)$. Therefore $\underline{\gamma}_{1}(Z)$ depends only on the isomorphism $\bar{k}(X) \cong k(Y)$, which $Z$ induces. From now on we fix such an isomorphism and simply write $\gamma_{1}$.

Now choose $f_{2}: X_{2} \rightarrow X$ and $g_{2}: Y_{2} \rightarrow Y$ finite and surjective, with $X_{2}, Y_{2}$ smooth and integral and $\operatorname{deg} f_{2}, \operatorname{deg} g_{2} \in k^{*}$. Define $\underline{\alpha}_{2}, \underline{\beta}_{2}$ and $\underline{\gamma}_{2}$ as above (in the above formulas replace 1 by 2 ) and set

$$
\begin{array}{lll}
\underline{\alpha}_{12}:=\left(\operatorname{deg} f_{1}\right)^{-1} \rho_{H} \circ \operatorname{cl}\left(\left[X_{1} \times_{X} X_{2}\right]\right), & \underline{\alpha}_{21}:=\left(\operatorname{deg} f_{2}\right)^{-1} \rho_{H} \circ \operatorname{cl}\left(\left[X_{2} \times_{X} X_{1}\right]\right) \\
\underline{\beta}_{12}:=\left(\operatorname{deg} g_{1}\right)^{-1} \rho_{H} \circ \operatorname{cl}\left(\left[Y_{1} \times_{Y} Y_{2}\right]\right), & \underline{\beta}_{21}:=\left(\operatorname{deg} g_{2}\right)^{-1} \rho_{H} \circ \operatorname{cl}\left(\left[Y_{2} \times_{Y} Y_{1}\right]\right) .
\end{array}
$$

Then one checks as in the proof of Claim 1 that $\underline{\alpha}_{12}: H^{i}\left(X_{1}\right) \rightarrow H^{i}\left(X_{2}\right)$ induces an isomorphism $\underline{\alpha}_{1} H^{i}\left(X_{1}\right) \stackrel{\simeq}{\rightarrow} \underline{\alpha}_{2} H^{i}\left(X_{2}\right)$ with inverse $\underline{\alpha}_{21}$ and $\underline{\beta}_{12}: H^{i}\left(Y_{1}\right) \rightarrow H^{i}\left(Y_{2}\right)$ induces an isomorphism $\beta_{1} H^{i}\left(Y_{1}\right) \stackrel{\simeq}{\rightarrow} \beta_{2} H^{i}\left(Y_{2}\right)$ with inverse $\beta_{21}$. Further one checks that $\underline{\beta}_{12} \circ \underline{\gamma}_{1} \circ \underline{\alpha}_{1}=\underline{\gamma}_{2} \circ \underline{\alpha}_{12} \circ \underline{\alpha}_{1}$. Thus we obtain the commutative diagram

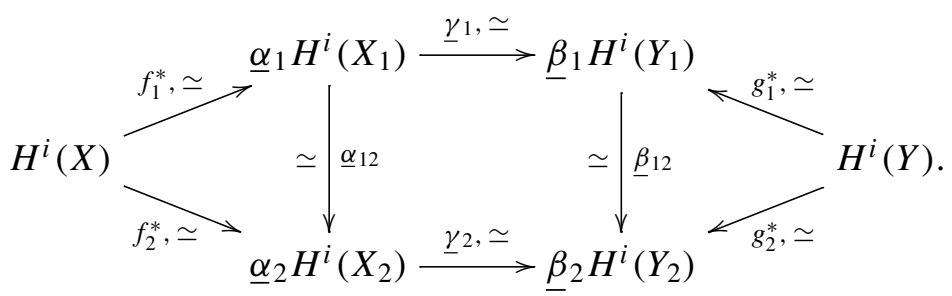


Therefore the isomorphisms of Claim 1 do not depend on the choice of $f_{1}$ and $g_{1}$. This proves Claim 2 and also the theorem in the case $S=\operatorname{Spec} k$.

Finally, consider the case of a general basis $S$. Choose $Z$ as in Definition 3.2.7 and choose integral, smooth schemes $X^{\prime}$ and $Y^{\prime}$ with finite, surjective morphisms $f: X^{\prime} \rightarrow X$ and $g: Y^{\prime} \rightarrow Y$ whose degree is invertible in $k$. For $U \subset S$ open denote by $X_{U}, f_{U}$, etc. the pull-backs over $U$. By Proposition 4.2.8, the pull-back $f_{U}^{*}$ realizes $H^{i}\left(X_{U}, \mathcal{O}_{X_{U}} \oplus \omega_{X_{U}}\right)$ as a direct summand of $H^{i}\left(X_{U}^{\prime}, \mathcal{O}_{X_{U}^{\prime}} \oplus \omega_{X_{U}^{\prime}}\right)$. This is clearly compatible with restrictions along opens $V \subset U \subset S$ and thus the pull-back $f^{*}$ realizes $R^{i} \pi_{X *}\left(O_{X} \oplus \omega_{X}\right)$ as a direct summand of the $\mathscr{O}_{S}$-module $R^{i} \pi_{X *} f_{*}\left(\mathscr{O}_{X^{\prime}} \oplus \omega_{X^{\prime}}\right)$. In the same way, $g^{*}$ realizes $R^{i} \pi_{Y *}\left(\mathcal{O}_{Y} \oplus \omega_{Y}\right)$ as a direct summand of the $\mathscr{O}_{S^{-}}$-module $R^{i} \pi_{Y *} g_{*}\left(\mathbb{O}_{Y^{\prime}} \oplus \omega_{Y^{\prime}}\right)$. Further, by the case $S=\operatorname{Spec} k$ considered above, the map

$$
(\operatorname{deg} f)^{-1} \rho_{H} \circ \operatorname{cl}\left(\left[Z_{U}^{\prime}\right]\right): H^{*}\left(X_{U}^{\prime}, \mathcal{O}_{X_{U}^{\prime}} \oplus \omega_{X_{U}^{\prime}}\right) \rightarrow H^{*}\left(Y_{U}^{\prime}, \mathscr{O}_{Y_{U}^{\prime}} \oplus \omega_{Y_{U}^{\prime}}\right)
$$

induces an isomorphism between $H^{*}\left(X_{U}, \mathrm{O}_{X_{U}} \oplus \omega_{X_{U}}\right)$ and $H^{*}\left(Y_{U}, \mathcal{O}_{Y_{U}} \oplus \omega_{Y_{U}}\right)$. Write $\left[Z^{\prime}\right]=\sum_{T} n_{T}[T]$, where the sum is over the irreducible components of $Z^{\prime}$. Then the collection $\left\{(\operatorname{deg} f)^{-1} \rho_{H} \circ \operatorname{cl}\left(\left[Z_{U}^{\prime}\right]\right) \mid U \subset S\right\}$ induces a morphism of $\mathrm{O}_{S}$-modules (by Proposition 3.2.4)

$$
\rho_{H}\left(Z^{\prime} / S\right):=\sum_{T} n_{T} \rho_{H}(T / S): R^{i} \pi_{X *} f_{*}\left(\mathbb{O}_{X^{\prime}} \oplus \omega_{X^{\prime}}\right) \rightarrow R^{i} \pi_{Y *} g_{*}\left(\mathcal{O}_{Y^{\prime}} \oplus \omega_{Y^{\prime}}\right),
$$

which by the above induces an isomorphism

$$
R^{i} \pi_{X *}\left(\mathrm{O}_{X} \oplus \omega_{X}\right) \stackrel{\simeq}{\rightarrow} R^{i} \pi_{Y *}\left(\mathscr{O}_{Y} \oplus \omega_{Y}\right) .
$$

Claim 2 implies that (4.3.10) depends only on the $\mathscr{O}_{S, \eta}$-isomorphism $k(X) \cong k(Y)$ induced by $Z$.

Remark 4.3.11. Theorem 4.3.1 implies Theorem 3.2.8 and Theorem 3.2.14.

Corollary 4.3.12. Let $\pi: X \rightarrow Y$ be a birational and proper morphism between integral schemes over a perfect field $k$. Assume $X$ and $Y$ are tame quotients. Then $\pi^{*}$ induces isomorphisms

$$
R \pi_{*} \mathrm{O}_{X} \cong \mathrm{O}_{Y} \text { and } R \pi_{*} \omega_{X} \cong \omega_{Y} .
$$

Proof. In Theorem 4.3.1 take $S=Y, \pi_{X}=\pi$ and $\pi_{Y}=\mathrm{id}_{Y}$.

4.4. Open questions. Questions in $\operatorname{char}(k)=p$.

4.4.1. Do the statements in Theorem 3.2.8 and Theorem 3.2.14 hold when $k$ is not perfect and smooth is replaced by regular?

4.4.2. Let $f: Y \rightarrow X$ be a surjective projective morphism with connected fibres between smooth varieties $Y$ and $X$. Is $R^{\operatorname{dim}(Y)-\operatorname{dim}(X)} f_{*} \omega_{Y}=\omega_{X}$ ? In $\operatorname{char}(k)=0$ this holds by [Kollár 1986, Proposition 7.6]. 
4.4.3. Let $f: Y \rightarrow X$ be a surjective projective morphism with connected fibres between smooth varieties $Y, X$. Is $R^{e} f_{*} \omega_{Y}=0$ for $e>\operatorname{dim}(Y)-\operatorname{dim}(X)$ ? In $\operatorname{char}(k)=0$ this holds by [Kollár 1986, Theorem 2.1(ii)].

\section{Appendix}

All schemes in this appendix are assumed to be finite-dimensional and noetherian.

A.1. Local Cohomology. Let $Y=\operatorname{Spec} B$ be an affine scheme and $X \subset Y$ a closed subscheme of pure codimension $c$, defined by the ideal $I \subset B$. We assume that there exists a $B$-regular sequence $t=t_{1}, \ldots, t_{c} \in I$ with $\sqrt{(t)}=\sqrt{I}$, where $(t)$ denotes the ideal $\left(t_{1}, \ldots, t_{c}\right) \subset B$. We denote by $K^{\bullet}(t)$ the Koszul complex of the sequence $t$, that is, $K^{-q}(t)=K_{q}(t)=\bigwedge^{q} B^{c}$ for $q=0, \ldots, c$, and if $\left\{e_{1}, \ldots, e_{c}\right\}$ is the standard basis of $B^{c}$ and $e_{i_{1}, \ldots, i_{q}}:=e_{i_{1}} \wedge \cdots \wedge e_{i_{q}}$, then the differential is given by

$$
d_{K}^{-q}\left(e_{i_{1}, \ldots, i_{q}}\right)=d_{q}^{K \cdot}\left(e_{i_{1}, \ldots, i_{q}}\right)=\sum_{j=1}^{q}(-1)^{j+1} t_{i_{j}} e_{i_{1}, \ldots, \hat{i_{j}}, \ldots i_{q}} .
$$

For any $B$-module $M$ we define the complex

$$
K^{\bullet}(t, M):=\operatorname{Hom}_{B}\left(K^{-\bullet}(t), M\right),
$$

and denote its $n$-th cohomology by $H^{n}(t, M)$. The map

$$
\operatorname{Hom}_{B}\left(\bigwedge^{c} B^{c}, M\right) \rightarrow M /(t) M, \quad \varphi \mapsto \varphi\left(e_{1, \ldots, c}\right)
$$

induces a canonical isomorphism $H^{c}(t, M) \simeq M /(t) M$.

If $t$ and $t^{\prime}$ are two sequences as above with $\left(t^{\prime}\right) \subset(t)$, then there exists a $c \times c$ matrix $T$ with coefficients in $B$ such that $t^{\prime}=T t$ and $T$ induces a morphism of complexes $K^{\bullet}\left(t^{\prime}\right) \rightarrow K^{\bullet}(t)$, which is the unique (up to homotopy) morphism lifting the natural map $B /\left(t^{\prime}\right) \rightarrow B /(t)$. Furthermore we observe that, for any pair of sequences $t$ and $t^{\prime}$ as above, there exists an $N \geq 0$ such that $\left(t^{N}\right) \subset\left(t^{\prime}\right)$, where $t^{N}$ denotes the sequence $t_{1}^{N}, \ldots, t_{c}^{N}$. Thus the sequences $t$ form a directed set and $H^{c}(t, M) \rightarrow H^{c}\left(t^{\prime}, M\right)$ for $\left(t^{\prime}\right) \subset(t)$ becomes a direct system. It follows from [Grothendieck 1968, Exposé II, Proposition 5] that we have an isomorphism

$$
\underset{t}{\lim } M /(t) M=\underset{t}{\lim } H^{c}(t, M) \cong H_{X}^{c}(Y, \tilde{M}),
$$

where the limit is over all $B$-regular sequences $t=t_{1}, \ldots, t_{c}$ in $B$ with $V((t))=X$ and $\widetilde{M}$ is the sheaf associated to $M$. We denote by $\left[\begin{array}{c}m \\ t\end{array}\right]$ the image of $m \in M$ under the composition

$$
M \rightarrow M /(t) M \rightarrow H^{c}(t, M) \rightarrow H_{X}^{c}(Y, \tilde{M})
$$


It is a consequence of the explanations above that we have the following properties:

(1) Let $t$ and $t^{\prime}$ be two sequences as above with $\left(t^{\prime}\right) \subset(t)$. Let $T$ be a $c \times c$-matrix with $t^{\prime}=T t$; then

$$
\left[\begin{array}{c}
\operatorname{det}(T) m \\
t^{\prime}
\end{array}\right]=\left[\begin{array}{c}
m \\
t
\end{array}\right] .
$$

(2) $\left[\begin{array}{c}m+m^{\prime} \\ t\end{array}\right]=\left[\begin{array}{c}m \\ t\end{array}\right]+\left[\begin{array}{c}m^{\prime} \\ t\end{array}\right]$ and $\left[\begin{array}{c}t_{i} m \\ t\end{array}\right]=0$ for all $i$.

(3) If $M$ is any $B$-module of finite rank, then

$$
H_{X}^{c}\left(Y, O_{Y}\right) \otimes_{B} M \stackrel{\simeq}{\longrightarrow} H_{X}^{c}(Y, \tilde{M}), \quad\left[\begin{array}{l}
b \\
t
\end{array}\right] \otimes m \mapsto\left[\begin{array}{c}
b m \\
t
\end{array}\right]
$$

is an isomorphism.

Remark A.1.1. Since for a $B$-regular sequence $t$ as above $K^{\bullet}(t) \rightarrow B /(t)$ is a free resolution, we have an isomorphism for all $n$, given by

$$
\operatorname{Ext}^{n}(B /(t), M) \simeq H^{n}\left(\operatorname{Hom}_{B}^{\bullet}\left(K^{\bullet}(t), M\right)\right) .
$$

We also have an isomorphism

$$
\operatorname{Hom}_{B}^{\bullet}\left(K^{\bullet}(t), M\right) \simeq K^{\bullet}(t, M),
$$

which is given by multiplication with $(-1)^{n(n+1) / 2}$ in degree $n$. We obtain an isomorphism

$$
\psi_{t, M}: \operatorname{Ext}^{c}(B /(t), M) \stackrel{\simeq}{\rightarrow} H^{c}(t, M)=M /(t) M,
$$

which has the sign $(-1)^{c(c+1) / 2}$ in it. In particular, under the composition

$$
\operatorname{Ext}^{c}(B /(t), M) \stackrel{\psi_{t, M}}{\longrightarrow} M /(t) M \rightarrow H_{X}^{c}(Y, \tilde{M}),
$$

the class of a map $\varphi \in \operatorname{Hom}_{B}\left(\bigwedge^{c} B^{c}, M\right)$ is sent to

$$
(-1)^{c(c+1) / 2}\left[\begin{array}{c}
\varphi\left(e_{1, \ldots, c}\right) \\
t
\end{array}\right] \text {. }
$$

Lemma A.1.2. Let $Y=\operatorname{Spec} B$ be as above, let $M$ be a quasicoherent sheaf on $Y$, let $c \geq 0$ and let $t_{1}, \ldots, t_{c+1}$ be a $B$-regular sequence. Set $X^{\prime}:=V\left(t_{1}, \ldots, t_{c+1}\right) \subset$ $X:=V\left(t_{1}, \ldots, t_{c}\right)$. Let $\partial: H_{X \backslash X^{\prime}}^{c}\left(Y \backslash X^{\prime}, \mathcal{M}\right) \rightarrow H_{X^{\prime}}^{c+1}(Y, \mathcal{M})$ be the boundary map of the localization long exact sequence. Then

$$
\partial\left[\begin{array}{c}
m / t_{c+1} \\
t_{1}, \ldots, t_{c}
\end{array}\right]=\left[\begin{array}{c}
m \\
t_{1}, \ldots, t_{c}, t_{c+1}
\end{array}\right] .
$$


Proof. Let $M$ be the $B$-module of global sections of $\mathcal{M}$. By [Grothendieck 1968, Exposé II, Corollary 4] and Čech computations, we may identify

$$
H_{X \backslash X^{\prime}}^{c}\left(Y \backslash X^{\prime}, M\right)=\frac{M_{t_{1} \cdots t_{c} t_{c+1}}}{\sum_{i=1}^{c} M_{t_{1} \cdots \widehat{t}_{i} \cdots t_{c} t_{c+1}}}, \quad H_{X^{\prime}}^{c+1}(Y, M)=\frac{M_{t_{1} \cdots t_{c+1}}}{\sum_{i=1}^{c+1} M_{t_{1} \cdots \widehat{t}_{i} \cdots t_{c+1}}},
$$

and $\partial$ is the natural map from left to right. Under these identifications, the map $M_{t_{c+1}} /\left(t_{1}, \ldots, t_{c}\right)=H^{c}\left(K^{\bullet}(t, M)\right) \rightarrow H_{X \backslash X^{\prime}}^{c}(Y, M)$ sends the class of $m / t_{c+1}$, for $m \in M$, to the class of $\left(m / t_{c+1}\right) /\left(t_{1} \cdots t_{c}\right)$ and similarly for $M /\left(t_{1}, \ldots, t_{c+1}\right) \rightarrow$ $H_{X^{\prime}}^{c+1}(Y, M)$.

A.2. The trace for a regular closed embedding. We now explicitly describe the trace morphism for a regular closed embedding. This is well known and appears in various incarnations in the literature; see for example [Lipman 1984; Hübl and Seibert 1997, Section 4]. However in all the articles we are aware of, more elementary versions of duality theory are used (for example no derived categories appear). Since the compatibility of these theories with the one we are working with — namely the one developed in [Hartshorne 1966; Conrad 2000] — is not evident to us, and also to be sure about the signs, we recall the description of the trace in this situation.

Let $i: X \hookrightarrow Y$ be a closed immersion of pure codimension $c$ between two Gorenstein schemes and assume that the ideal sheaf $\mathscr{I}$ of $X$ is generated by a sequence $t=\left(t_{1}, \ldots, t_{c}\right)$ of global sections of $\mathrm{O}_{Y}$. Then the image of $t$ in any local ring of $Y$ is automatically a regular sequence. We denote by $K^{\bullet}(t)$ the sheafified Koszul complex of $t$ and set

$$
\omega_{X / Y}:=\bigwedge^{c} \mathcal{H}_{\mathrm{om}_{\mathrm{O}_{X}}\left(\mathscr{I} / \mathscr{\Phi}^{2}, \mathrm{O}_{X}\right)}
$$

The fundamental local isomorphism (see for example [Conrad 2000, 2.5]) gives an isomorphism in $D_{c}^{b}(Y)$

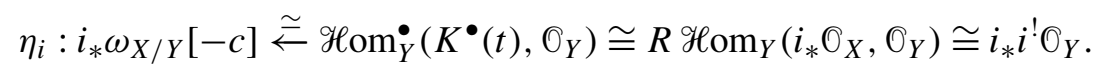

The first map is induced by

$$
\begin{aligned}
\operatorname{Hom}\left(\bigwedge^{c} \mathcal{O}_{Y}^{c}, \mathcal{O}_{Y}\right) & =\operatorname{Hom}^{c}\left(K^{\bullet}(t), \mathscr{O}_{Y}\right) \rightarrow i_{*} \omega_{X / Y}, \\
\varphi & \mapsto(-1)^{c(c+1) / 2} \varphi\left(e_{1, \ldots, c}\right) t_{1}^{\vee} \wedge \cdots \wedge t_{c}^{\vee} .
\end{aligned}
$$

(The reason for the sign is Remark A.1.1.) Composing the morphism $\eta_{i}$ with the trace $\operatorname{Tr}_{i}: i_{*} i ! \mathrm{O}_{Y} \rightarrow \mathrm{O}_{Y}$ (see for example [Conrad 2000, 3.4]), we obtain a morphism in $D_{\mathrm{c}}^{b}(Y)$

$$
i_{*} \omega_{X / Y}[-c] \stackrel{\eta_{i}}{\rightarrow} i_{*} i^{!} \mathfrak{O}_{Y} \stackrel{\mathrm{Tr}_{i}}{\rightarrow} \mathrm{O}_{Y}
$$


which factors in $D_{\mathrm{qc}}^{b}(Y)$ as

$$
i_{*} \omega_{X / Y}[-c] \stackrel{\eta_{i}}{\rightarrow} i_{*} i ! \widehat{O}_{Y} \stackrel{\operatorname{Tr}_{i}}{\rightarrow} R \underline{\Gamma_{X}} \mathcal{O}_{Y} .
$$

Lemma A.2.5. In the situation above there is a natural isomorphism

$$
R \underline{\Gamma}_{X} \bigcirc_{Y} \cong \mathscr{H}_{X}^{c}\left(\bigcirc_{Y}\right)[-c] \text { in } D_{\mathrm{qc}}^{b}(Y)
$$

and $\mathscr{H}^{c}((A .2 .4))$ is given by

$$
i_{*} \omega_{X / Y} \longrightarrow \mathscr{H}_{X}^{c}\left(\mathrm{O}_{Y}\right), \quad a t_{1}^{\vee} \wedge \cdots \wedge t_{c}^{\vee} \mapsto(-1)^{c(c+1) / 2}\left[\begin{array}{c}
\tilde{a} \\
t_{1}, \ldots, t_{c}
\end{array}\right],
$$

where $\tilde{a} \in \mathrm{O}_{Y}$ is any lift of $a \in \mathrm{O}_{X}$.

Proof. The first statement is equivalent to $\mathscr{H}_{X}^{i}\left(\mathscr{O}_{Y}\right)=0$, for $i \neq c$, and hence we may assume that $Y$ is affine. We have the vanishing for $i<c$ since $Y$ is $\mathrm{CM}$ by [Grothendieck 1968, Exposé III, Proposition 3.3] and for $i>c$ since the ideal of $X$ in $Y$ is generated by $c$ elements, which by a Čech argument implies that $H^{i}\left(Y \backslash X, \mathrm{O}_{Y}\right)=0$ for $i>c$.

We denote by $E^{\bullet}=E^{\bullet}\left(\mathscr{O}_{Y}\right)$ the Cousin complex of $\mathscr{O}_{Y}$; see e.g., [Hartshorne 1966, Chapter IV, Section 2]. In particular $E^{\bullet}$ is an injective resolution of $O_{Y}$ (since $Y$ is Gorenstein) and if $Y^{(c)}$ denotes the set of points of codimension $c$ in $Y$, then

$$
E^{q}=\bigoplus_{y \in Y^{(c)}} i_{y *} H_{y}^{q}\left(Y, O_{Y}\right)
$$

where $i_{y}: y \rightarrow Y$ is the inclusion and $H_{y}^{q}\left(Y, O_{Y}\right)=\operatorname{colim}_{y \in U} H_{\bar{y} \cap U}^{q}\left(Y, O_{Y}\right)$, the limit being over all open subsets $U \subset Y$ that contain $y$. We write $K^{\bullet}:=K^{\bullet}(t)$.

The trace $\operatorname{Tr}_{i}: i_{*} i^{!} O_{Y} \rightarrow O_{Y}$ is now induced by the "evaluation at 1 " morphism $\operatorname{Hom}^{\bullet}\left(i_{*} \mathrm{O}_{X}, E^{\bullet}\right) \rightarrow E^{\bullet}$. Furthermore the augmentation morphisms $K^{\bullet} \rightarrow i_{*} \mathrm{O}_{X}$ and $\mathrm{O}_{Y} \rightarrow E^{\bullet}$ induce quasiisomorphisms

$$
\operatorname{Hom}^{\bullet}\left(K^{\bullet}, \mathrm{O}_{Y}\right) \stackrel{\sim}{\rightarrow} \operatorname{Hom}^{\bullet}\left(K^{\bullet}, E^{\bullet}\right) \stackrel{\sim}{\leftarrow} \mathscr{H}^{\circ}{ }^{\bullet}\left(i_{*} \mathrm{O}_{X}, E^{\bullet}\right) .
$$

To prove the second statement, we may assume $a=1 \in \mathrm{O}_{X}$. We define $\alpha \in$ $\operatorname{Hom}^{c}\left(K^{\bullet}, \mathrm{O}_{Y}\right)=\mathscr{H o m}\left(\wedge^{c} \mathcal{O}_{Y}^{c}, \mathrm{O}_{Y}\right)$ by $\alpha\left(e_{1, \ldots, c}\right)=1$ and $\beta \in \operatorname{Hom}^{c}\left(i_{*} \widehat{O}_{X}, E^{\bullet}\right)=$ $\operatorname{Hom}\left(i_{*} \mathcal{O}_{X}, E^{c}\right)$ by

$$
\beta(1)=\left(\beta_{y}\right) \in E^{c}=\bigoplus_{y \in Y^{(c)}} i_{y *} H_{y}^{c}\left(Y, O_{Y}\right)
$$

with

$$
\beta_{y}= \begin{cases}{\left[\begin{array}{c}
1 \\
t_{1}, \ldots, t_{c}
\end{array}\right]} & \text { if } y \text { is a generic point of } X \\
0 & \text { otherwise }\end{cases}
$$


Then

$$
\operatorname{Tr}_{i}(\bar{\beta})=\left[\begin{array}{c}
1 \\
t_{1}, \ldots, t_{c}
\end{array}\right] \in \mathscr{H}_{X}^{c}\left(\mathscr{O}_{Y}\right),
$$

where $\bar{\beta}$ is the residue class of $\beta$ in $\mathscr{H}^{c}\left(\mathscr{H o m}^{\bullet}\left(i_{*} \mathrm{O}_{X}, E^{\bullet}\right)\right)$ and

$$
\eta_{i}(\alpha)=(-1)^{c(c+1) / 2} t_{1}^{\vee} \wedge \cdots \wedge t_{c}^{\vee} \in i_{*} \omega_{X / Y} .
$$

Thus the second statement of the lemma follows if we can show that the images of $\alpha$ and $\beta$ in $\mathscr{H o m}^{c}\left(K^{\bullet}, E^{\bullet}\right)$ differ by an element in $d_{\mathscr{L o m}}^{c-1}\left(\mathscr{H o m}^{c-1}\left(K^{\bullet}, E^{\bullet}\right)\right)$.

For $j=0, \ldots, c-1$, we define

$$
\gamma^{c-1-j}=\left(\gamma_{y}^{c-1-j}\right) \in E^{c-1-j}=\bigoplus_{y \in Y^{(c-1-j)}} i_{y *} H_{y}^{c-1-j}\left(Y, \mathrm{O}_{Y}\right)
$$

by

$$
\gamma_{y}^{c-1-j}:= \begin{cases}{\left[\begin{array}{c}
1 / t_{c-j} \\
t_{1}, \ldots, t_{c-j-1}
\end{array}\right]} & \text { if } y \in Y^{(c-j-1)} \cap V\left(t_{1}, \ldots, t_{c-1-j}\right), \\
0 & \text { otherwise. }\end{cases}
$$

In particular $\gamma^{0}=1 / t_{1} \in \bigoplus_{i} H_{\eta_{i}}^{0}\left(Y, O_{Y}\right)=\oplus_{i} k\left(\eta_{i}\right)$, with $\eta_{i}$ the generic points of $Y$. Notice that (by Lemma A.1.2)

$$
\begin{aligned}
d_{E} \gamma^{c-1-j} & =t_{c-j+1} \gamma^{c-j} & & \text { for all } j \geq 1, \\
t_{i} \gamma^{c-1-j} & =0 & & \text { for all } j \geq 0, i \in\{1, \ldots, c-1-j\} .
\end{aligned}
$$

Further define

$$
\psi=\left(\psi_{0}, \ldots, \psi_{c-1}\right) \in \operatorname{Hom}^{c-1}\left(K^{\bullet}, E^{\bullet}\right)=\bigoplus_{j=0}^{c-1} \mathscr{H} \mathrm{om}\left(K_{j}, E^{c-1-j}\right)
$$

by

$$
\psi_{j}\left(e_{i_{1}, \ldots, i_{j}}\right)= \begin{cases}(-1)^{j(c+j)} \gamma^{c-1-j} & \text { if }\left(i_{1}, \ldots, i_{j}\right)=(c+1-j, \ldots, c), \\ 0 & \text { otherwise. }\end{cases}
$$

By definition and (A.2.10) we have

$$
t_{i_{q}} \psi_{j-1}\left(e_{i_{1}, \ldots, \hat{i_{q}}, \ldots, i_{j}}\right) \neq 0 \text { if } q \neq 1 \text { or }\left(i_{1}, \ldots, i_{j}\right) \neq(c+1-j, \ldots, c) \text {. }
$$

Now we calculate the boundary of $\psi$, $d_{\mathscr{H} \circ \mathrm{m}}^{c-1} \psi=\left(d_{E}^{c-1} \circ \psi_{0}, \ldots, d_{E}^{c-1-j} \circ \psi_{j}+(-1)^{c} \psi_{j-1} \circ d_{j}^{K}, \ldots,(-1)^{c} \psi_{c-1} \circ d_{c}^{K}\right)$. 1st Case: $j=0$.

$$
d_{E}^{c-1} \circ \psi_{0}(1)=d_{E}^{c-1} \gamma^{c-1}=\beta(1) \quad \text { by (A.2.9). }
$$

2nd Case: $1 \leq j \leq c-2$. By (A.2.11) and the definition of $\psi$, we have

$$
\left(d_{E}^{c-1-j} \circ \psi_{j}+(-1)^{c} \psi_{j-1} \circ d_{j}^{K}\right)\left(e_{i_{1}, \ldots, i_{j}}\right)=0
$$


if $\left(i_{1}, \ldots, i_{j}\right) \neq(c+1-j, \ldots, c)$ and otherwise

$$
\begin{aligned}
\left(d_{E}^{c-1-j}\right. & \left.\circ \psi_{j}+(-1)^{c} \psi_{j-1} \circ d_{j}^{K}\right)\left(e_{c+1-j, \ldots, c}\right) \\
& =(-1)^{j(c+j)} d_{E}^{c-1-j} \gamma^{c-1-j}+(-1)^{c} t_{c+1-j} \psi_{j-1}\left(e_{c+2-j, \ldots, c}\right) \\
& =(-1)^{j(c+j)}\left(d_{E}^{c-1-j} \gamma^{c-1-j}-t_{c-j+1} \gamma^{c-j}\right)=0 \text { by (A.2.9). }
\end{aligned}
$$

3rd Case: $j=c-1$. By (A.2.11), we have

$$
(-1)^{c} \psi_{c-1}\left(d_{c}^{K}\left(e_{1, \ldots, c}\right)\right)=(-1)^{c} t_{1} \psi_{c-1}\left(e_{2, \ldots, c}\right)=-1=-\alpha\left(e_{1, \ldots, c}\right) .
$$

All in all we obtain

$$
d_{\mathscr{H o m}}^{c-1}(\psi)=(\beta, 0, \ldots, 0,-\alpha)
$$

Lemma A.2.12. Let $S$ be a Gorenstein scheme and $i: X \hookrightarrow Y$ a closed immersion between smooth, separated and equidimensional $S$-schemes with structure maps $\pi_{X}: X \rightarrow S$ and $\pi_{Y}: Y \rightarrow S$ and denote by $d_{X / S}$ and $d_{Y / S}$ their relative dimensions. We set $\omega_{X / S}:=\Omega_{X / S}^{d_{X / S}}, \omega_{Y / S}:=\Omega_{Y / S}^{d_{Y / S}}$ and $c=d_{Y / S}-d_{X / S}$. Assume that the ideal sheaf of $X$ in $Y$ is generated by a sequence $t=t_{1}, \ldots, t_{c}$ of global sections of $\mathrm{O}_{Y}$. Define a morphism $\iota_{X}$ by

$$
\iota_{X}: i_{*} \omega_{X / S} \rightarrow \mathscr{H}_{X}^{c}\left(\omega_{Y / S}\right), \quad \alpha \mapsto(-1)^{c}\left[\begin{array}{c}
d t \tilde{\alpha} \\
t
\end{array}\right],
$$

with $\tilde{\alpha} \in \Omega_{Y / S}^{d_{X / S}}$ any lift of $\alpha$ and $d t=d t_{1} \wedge \cdots \wedge d t_{c}$. Then the following diagram in $D_{\mathrm{qc}}^{b}\left(\mathrm{O}_{Y}\right)$ is commutative:

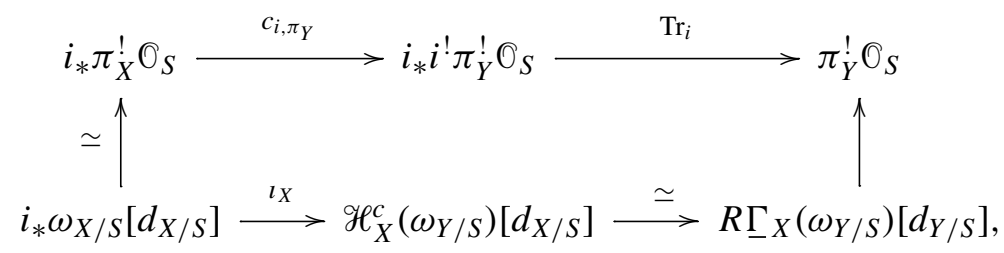

where the vertical map on the left is the well-known canonical isomorphism (see [Conrad 2000, (3.3.21)]), the vertical map on the right is the composition of the forget supports map $R \underline{\Gamma}_{X}\left(\omega_{Y / S}\right)\left[d_{Y / S}\right] \rightarrow \omega_{Y / S}\left[d_{Y / S}\right]$ with the canonical isomorphism $\omega_{Y / S}\left[d_{Y / S}\right] \cong \pi_{Y}^{!} O_{S}$ and $c_{i, \pi_{Y}}: \pi_{X}^{!} \cong i^{!} \pi_{Y}^{!}$is the canonical isomorphism [Conrad 2000, (3.3.14)].

Proof. Let $\Phi \subset O_{Y}$ be the ideal sheaf of $X$. As above we write

$$
\omega_{X / Y}:=\bigwedge^{c} \operatorname{Hom}_{\mathcal{O}_{X}}\left(\mathscr{I} / \mathscr{I}^{2}, \mathscr{O}_{X}\right)
$$

Further let $\tau_{Y}: \pi_{Y}^{!} O_{S} \cong \omega_{Y / S}\left[d_{Y / S}\right]$ and $\tau_{X}: \pi_{X}^{!} O_{S} \cong \omega_{X / S}\left[d_{X / S}\right]$ be the canonical isomorphisms and $\eta_{i}: \omega_{X / Y}[-c] \cong i^{!} \sigma_{Y}$ the fundamental local isomorphism 
(A.2.1). Consider the following diagram in $D_{\mathrm{c}}^{b}\left(\mathrm{O}_{Y}\right)$ :

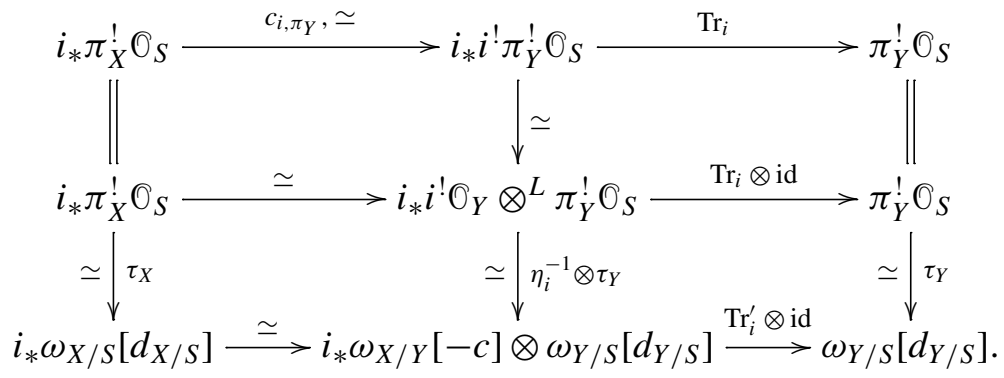

Here some explanations: The middle horizontal arrow on the left is defined such that the upper left square commutes. We have a canonical identification $i_{*} i^{!}(\cdot)=$ $R \operatorname{Hom}_{\mathscr{O}_{Y}}\left(i_{*} \mathrm{O}_{X},(\cdot)\right)$ and since $\pi_{Y}^{!} \mathrm{O}_{S}$ is isomorphic to a shifted locally free $\mathscr{O}_{Y^{-}}$

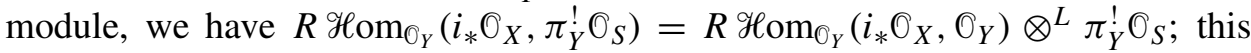
defines the upper vertical arrow in the middle. Furthermore $\operatorname{Tr}_{i}: i_{*} i^{\prime}(\cdot) \rightarrow(\cdot)$ may be identified with $R \operatorname{Hom}_{\mathscr{O}_{Y}}\left(i_{*} \mathrm{O}_{X}, \cdot\right) \rightarrow(\cdot)$ given by the evaluation at 1 . This shows, that in the diagram above the upper square on the right commutes. The map $\operatorname{Tr}_{i}^{\prime}: i_{*} \omega_{X / Y}[-c] \rightarrow \mathrm{O}_{Y}$ on the right bottom is the composition (A.2.3) and thus the lower square on the right commutes by definition. The horizontal isomorphism on the lower left is given by (see [Conrad 2000, page 29, 30(c) and (2.2.6)])

$$
\begin{aligned}
i_{*} \omega_{X / S}\left[d_{X / S}\right] & \rightarrow i_{*} \omega_{X / Y}[-c] \otimes \omega_{Y / S}\left[d_{Y / S}\right], \\
\alpha & \mapsto\left(t_{1}^{\vee} \wedge \cdots \wedge t_{c}^{\vee}\right) \otimes d t_{c} \wedge \cdots \wedge d t_{1} \wedge \tilde{\alpha},
\end{aligned}
$$

with $\tilde{\alpha} \in \Omega_{Y / S}^{d_{X / S}}$ any lift of $\alpha$. That the square on the lower left commutes follows from [Conrad 2000, Theorem 3.3.1, (3.3.27)] and [Conrad 2000, Lemma 3.5.3]. (Notice that by [Conrad 2001, p. 5, pp. 160-164] the statement of [Conrad 2000, Lemma 3.5.3] should be "(3.5.8) is equal to (3.5.7)" instead of "(3.5.8) is equal to $(-1)^{n(N-n)}$ times (3.5.7)".) Thus the whole diagram commutes. The upper line equals the upper line in (A.2.13) and the lower line factors as the composition

$$
\begin{aligned}
i_{*} \omega_{X / S}\left[d_{X / S}\right] \rightarrow & i_{*} \omega_{X / Y}[-c] \otimes \omega_{Y / S}\left[d_{Y / S}\right] \\
& \stackrel{\operatorname{tr}_{i} \otimes \mathrm{id}}{\longrightarrow} \mathscr{H}_{X}^{c}\left(\mathcal{O}_{Y}\right)[-c] \otimes \omega_{Y / S}\left[d_{Y / S}\right] \simeq \mathscr{H}_{X}^{c}\left(\omega_{Y / S}\right)\left[d_{X / S}\right]
\end{aligned}
$$

with the natural map

$$
\mathscr{H}_{X}^{c}\left(\omega_{Y / S}\right)\left[d_{X / S}\right] \simeq R \underline{\Gamma}_{X}\left(\omega_{Y / S}\right)\left[d_{Y / S}\right] \rightarrow \omega_{Y / S}\left[d_{Y / S}\right] .
$$

Here $\operatorname{tr}_{i}$ denotes the composition of $R \underline{\Gamma}_{X}\left(\operatorname{Tr}_{i}^{\prime}\right)$ with the isomorphism $R \Gamma_{X}\left(\mathcal{O}_{Y}\right) \cong$ $\mathscr{H}_{X}^{c}\left(\mathrm{O}_{Y}\right)[-c]$. Thus the lemma is proved once we know that (A.2.16) equals $l_{X}$. 
But by Lemma A.2.5 the map $\operatorname{tr}_{i}$ is given by

$$
i_{*} \omega_{X / Y} \rightarrow \mathscr{H}_{X}^{c}\left(\mathbb{O}_{Y}\right), \quad t_{1}^{\vee} \wedge \cdots \wedge t_{c}^{\vee} \mapsto(-1)^{c(c+1) / 2}\left[\begin{array}{l}
1 \\
t
\end{array}\right] .
$$

Together with (A.2.15) we obtain that (A.2.16) is given by

$$
\begin{aligned}
\alpha & \mapsto\left(t_{1}^{\vee} \wedge \cdots \wedge t_{c}^{\vee}\right) \otimes d t_{c} \wedge \cdots \wedge d t_{1} \wedge \tilde{\alpha} \\
& \mapsto(-1)^{c(c+1) / 2}\left[\begin{array}{l}
1 \\
t
\end{array}\right] \otimes d t_{c} \wedge \cdots \wedge d t_{1} \wedge \tilde{\alpha}=(-1)^{c}\left[\begin{array}{c}
d t \tilde{\alpha} \\
t
\end{array}\right],
\end{aligned}
$$

which by definition equals $l_{X}$. This proves the lemma.

\section{A.3. The trace for a finite and surjective morphism.}

1.3.1. Let $S$ be a Gorenstein scheme and $f: X \rightarrow Y$ a finite and surjective morphism of smooth, separated and equidimensional $S$-schemes, both of which have relative dimension $n$. We denote by $\pi_{X}: X \rightarrow S$ and $\pi_{Y}: Y \rightarrow S$ the respective structure maps. Then we define the trace map

$$
\tau_{f}^{n}: f_{*} \omega_{X / S} \rightarrow \omega_{Y / S}
$$

to be the composition

$$
f_{*} \omega_{X / S} \cong R f_{*} \pi_{X}^{!} \mathrm{O}_{S}[-n] \stackrel{\simeq c_{f, \pi_{Y}}}{\longrightarrow} R f_{*} f^{!} \pi_{Y}^{!} \mathrm{O}_{S}[-n] \stackrel{\operatorname{Tr}_{f}}{\longrightarrow} \pi_{Y}^{!} \mathrm{O}_{S}[-n] \cong \omega_{Y / S} .
$$

In the lemma below we give a well-known explicit description of this trace map, for which we could not find an appropriate reference. There are well-studied ad hoc definitions of this trace map not using the machinery of duality theory (see for example [Kunz 1986, §16]), but it is a priori not clear that these construction coincide with the one above.

Lemma A.3.3. Let $f: X \rightarrow Y$ be as above and assume it factors as

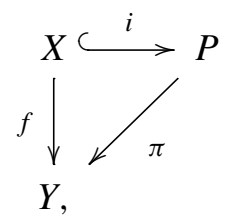

where $\pi$ is smooth and separated of pure relative dimension $d$ and $i$ is a closed immersion whose ideal sheaf $\mathscr{\neg} \subset \mathrm{O}_{P}$ is generated by global sections $t_{1}, \ldots, t_{d} \in$ $\Gamma\left(P, O_{P}\right)$. Then for any local section $\alpha \in f_{*} \omega_{X / S}$, we have a formula for $\tau_{f}^{n}(\alpha)$ : Let $\tilde{\alpha} \in \Omega_{P / S}^{n}$ be any lift of $\alpha$ and write

$$
i^{*}\left(d t_{d} \wedge \cdots \wedge d t_{1} \wedge \tilde{\alpha}\right)=\sum_{j} i^{*} \gamma_{j} \otimes f^{*} \beta_{j}, \quad \text { where } \gamma_{j} \in \omega_{P / Y}, \beta_{j} \in \omega_{Y / S}
$$


in $i^{*} \Omega_{P / S}^{n+d}=i^{*} \omega_{P / S}=i^{*}\left(\omega_{P / Y}\right) \otimes f^{*} \omega_{Y / S}$. Then

$$
\tau_{f}^{n}(\alpha)=(-1)^{d(d-1) / 2} \sum_{j} \operatorname{Res}_{P / Y}\left[\begin{array}{c}
\gamma_{j} \\
t_{1}, \ldots, t_{d}
\end{array}\right] \beta_{j} \in \omega_{Y / S},
$$

where $\operatorname{Res}_{P / Y}\left[\begin{array}{c}\gamma_{j} \\ t_{1}, \ldots, t_{d}\end{array}\right] \in \mathcal{O}_{Y}$ is the residue symbol defined in [Conrad 2000, (A.1.4)]. Proof. The proof is a collection of compatibility statements from [Conrad 2000]. First we collect some notation.

(1) $\zeta_{i, \pi_{P}}^{\prime}: \omega_{X / S} \rightarrow \omega_{X / P} \otimes i^{*} \omega_{P / S}$ is defined in [Conrad 2000, pp. 29-30, (c)] and sends $\alpha$ to $\left(t_{1}^{\vee} \wedge \cdots \wedge t_{d}^{\vee}\right) \otimes i^{*}\left(d t_{d} \wedge \cdots \wedge d t_{1} \wedge \tilde{\alpha}\right)$, where we identify $\omega_{X / P}=\bigwedge^{d}\left(\mathscr{\Phi} / \mathscr{I}^{2}\right)^{\vee}$.

(2) $\eta_{i}: \mathscr{E} x t_{P}^{d}\left(i_{*} \mathscr{O}_{X}, \cdot\right) \stackrel{\simeq}{\rightarrow} \omega_{X / P} \otimes i^{*}(\cdot)$ is the fundamental local isomorphism [Conrad 2000, (2.5.1)].

(3) For a smooth and separated morphism of pure relative dimension $n$ between two schemes $g: V \rightarrow W, e_{g}: g^{!} \stackrel{\simeq}{\longrightarrow} g^{\#}=\omega_{V / W}[n] \otimes^{L}(\cdot)$ denotes the natural transformation [Conrad 2000, (3.3.21)].

(4) In case $g$ as above factors as $g=h \circ i$ with $i: V \rightarrow Z$ finite and $h: Z \rightarrow W$ smooth, $\psi_{i, h}: g^{\#} \stackrel{\simeq}{\rightarrow} i^{\mathrm{b}} h^{\#}$ is the isomorphism defined in [Conrad 2000, (2.7.5)], where $i^{b}(\cdot)=i^{-1} R \operatorname{Hom}_{Z}\left(i_{*} \mathscr{O}_{V}, \cdot\right) \otimes_{i^{-1} i_{*} O_{V}} \mathscr{O}_{V}$ is defined in [Conrad 2000, (2.2.8)].

(5) $d_{f}: f^{!} \stackrel{\simeq}{\longrightarrow} f^{b}$ is the isomorphism defined in [Conrad 2000, (3.3.19)].

(6) $\operatorname{Tr}_{f}: f_{*} f^{!} \rightarrow$ id is the trace morphism defined in [Conrad 2000, 3.4], and $\operatorname{Trf}_{f}: f_{*} f^{b} \rightarrow$ id is the finite trace morphism defined in [Conrad 2000, (2.2.9)] and which is induced by evaluation at 1 .

Consider the diagram on page 773 . Let us describe the different squares and triangles in this diagram:

(1) The vertical isomorphism on the right in square 1 is immediate from the definition of $\pi_{Y}^{\#}$; the left vertical isomorphism is defined such that the square commutes.

(2) See [Conrad 2000, Theorem 3.5.1, Corollary 3.5.2] for the isomorphism in the lower right of square 2. The square commutes by [Conrad 2000, Lem. 3.5.3]. (By [Conrad 2001, comment to pp. 160-164] the last statement of [Conrad 2000, Lemma 3.5.3] should be "..., then (3.5.8) is equal to (3.5.7)".)

(3) Square 3 commutes by [Conrad 2000, (3.3.27)].

(4) The vertical isomorphism on the right of square 4 is induced by the natural isomorphism $\left(\pi \pi_{Y}\right)^{\#} \cong \pi_{Y}^{\#} \pi^{\#}$. For the commutativity of the square, see the discussion in [Conrad 2000, p. 83-84] (our case is point three). 


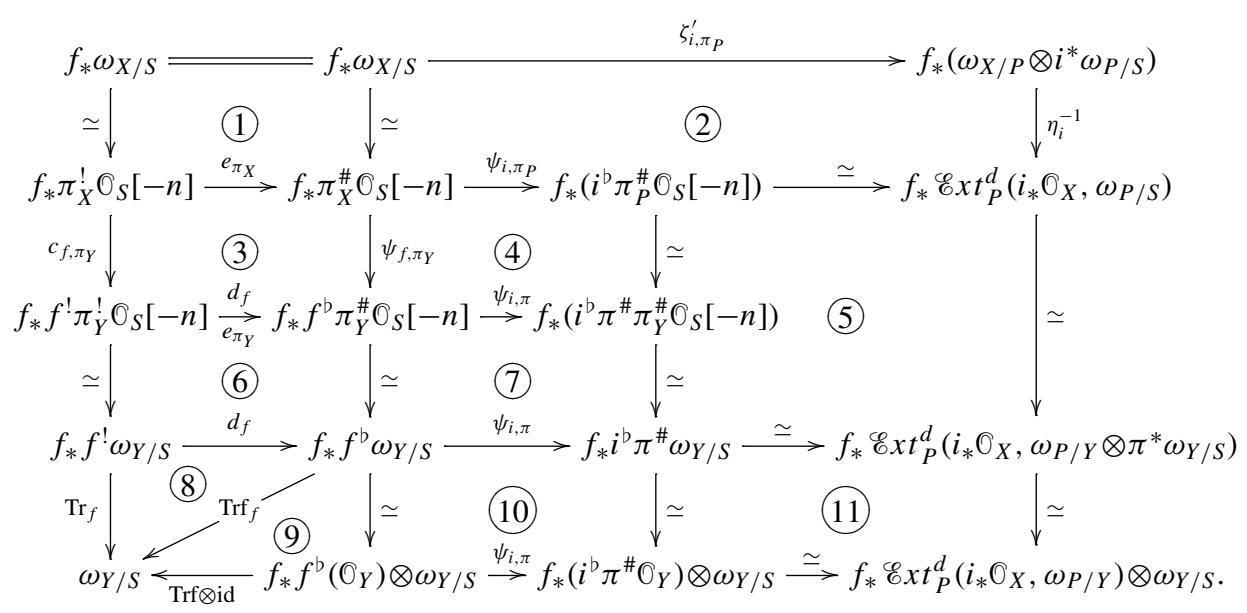

(5) The isomorphism on the right of square 5 is induced by the natural isomorphism $\omega_{P / S} \cong \omega_{P / Y} \otimes \pi^{*} \omega_{Y / S}$. The square commutes by the functoriality of the horizontal isomorphisms, which are just induced by taking the 0-th cohomology (the other cohomology groups being zero).

(6) The vertical isomorphism on the right of square 6 is induced by $\omega_{Y / S} \cong$ $\pi_{Y}^{!} O_{S}[-n] \cong \pi_{Y}^{\#} O_{S}[-n]$. Thus the square commutes by the functoriality of $d_{f}$.

(7) The vertical isomorphism on the right of square 7 is defined as above. Thus the square commutes by the functoriality of $\psi_{i, \pi}$.

(8) Triangle 8 commutes by [Conrad 2000, Lemma 3.4.3, (TRA2)].

(9) By [Hartshorne 1966, proof of Chapter III, Proposition 6.5], we may identify $f_{*} f^{b} \omega_{Y / S}$ with the sheaf $\operatorname{Hom}_{Y}\left(f_{*} O_{X}, \omega_{Y / S}\right)$ (since $\omega_{Y / S}$ is locally free) and $\operatorname{Trf}_{f}$ is given by evaluation at 1 . The vertical map on the right of triangle 9 is defined by the isomorphism $\mathscr{H}_{\operatorname{om}_{Y}}\left(f_{*} \mathcal{O}_{X}, \omega_{Y / S}\right) \cong \operatorname{Hom}_{Y}\left(f_{*} \mathrm{O}_{X}, \mathrm{O}_{Y}\right) \otimes \omega_{Y / S}$. The triangle thus obviously commutes.

(10) By [Conrad 2000, (2.8.3) and the paragraph after this, pp. 100-101] we have a commutative square

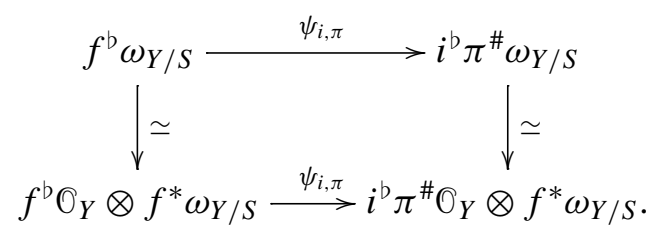

Applying $f_{*}$ to this diagram and using projection formula defines the commutative square 10 . 
(11) The horizontal maps in square 11 are induced by taking the 0 -th cohomology, the vertical maps are the natural isomorphisms $\left(\omega_{Y / S}\right.$ is locally free and projection formula). The commutativity of the diagram is clear.

(12) The isomorphism in the upper right of triangle 12 is induced by the isomorphism $\omega_{P / S} \cong \omega_{P / Y} \otimes \pi^{*} \omega_{Y / S}$ and the projection formula. The triangle commutes by [Conrad 2000, Theorem 2.5.2, 1].

Thus diagram on page 773 is commutative. The composition of the vertical maps along the left outer edge of the diagram equals $\tau_{f}^{n}$ by definition. The composition of the vertical maps along the right outer edge of the diagram is by [Conrad 2000, Theorem 2.5.2, 1] equal to the composition

$$
\begin{aligned}
f_{*}\left(\omega_{X / P} \otimes i^{*} \omega_{P / S}\right) & \stackrel{\simeq}{\longrightarrow} f_{*}\left(\omega_{X / P} \otimes i^{*} \omega_{P / Y}\right) \otimes \omega_{Y / S} \\
& \stackrel{\eta_{i}^{-1} \otimes \mathrm{id}}{\longrightarrow} f_{*} \mathscr{E} x t_{P}^{d}\left(i_{*} \mathscr{O}_{X}, \omega_{P / Y}\right) \otimes \omega_{Y / S} .
\end{aligned}
$$

All together we see that $\tau_{f}^{n}$ equals the composition

$$
\begin{aligned}
& f_{*} \omega_{X / S} \stackrel{\zeta_{i, \pi}^{\prime}}{\longrightarrow} f_{*}\left(\omega_{X / P} \otimes i^{*} \omega_{P}\right) \cong f_{*}\left(\omega_{X / P} \otimes i^{*} \omega_{P / Y}\right) \otimes \omega_{Y / S} \stackrel{\eta_{i}^{-1} \otimes \mathrm{id}}{\longrightarrow} \\
& f_{*}\left(i^{\mathrm{b}} \pi^{\#} \bigcirc_{Y}\right) \otimes \omega_{Y / S} \stackrel{\psi_{i, \pi}^{-1}}{\longrightarrow} f_{*} f^{\mathrm{b}} \mathcal{O}_{Y} \otimes \omega_{Y} \cong \operatorname{Hom}_{Y}\left(f_{*} \mathcal{O}_{X}, \mathcal{O}_{Y}\right) \otimes \omega_{Y / S} \stackrel{\text { eval. at } 1}{\longrightarrow} \omega_{Y / S} .
\end{aligned}
$$

Hence the claim follows from the definition of $\zeta_{i, \pi_{P}}^{\prime}$ (see (1) above) and the definition of the residue symbol in [Conrad 2000, (A.1.4)].

\section{Acknowledgments}

We are deeply grateful to Hélène Esnault for her encouragement and patience. We thank Manuel Blickle, Georg Hein and Eckart Viehweg for useful discussions. Finally, we thank the referee for a careful reading and for suggesting many improvements in the exposition.

\section{References}

[Conrad 2000] B. Conrad, Grothendieck duality and base change, Lecture Notes in Mathematics 1750, Springer, Berlin, 2000. MR 2002d:14025 Zbl 0992.14001

[Conrad 2001] B. Conrad, "Clarifications and corrections for [Conrad 2000]", 2001, available at http://math.stanford.edu/ conrad/papers/dualitycorrections.pdf.

[Conrad 2007] B. Conrad, "Deligne's notes on Nagata compactifications", J. Ramanujan Math. Soc. 22:3 (2007), 205-257. MR 2009d:14002 Zbl 1142.14001

[Fulton 1998] W. Fulton, Intersection theory, 2nd ed., Ergebnisse der Mathematik und ihrer Grenzgebiete (3) 2, Springer, Berlin, 1998. MR 99d:14003 Zbl 0885.14002

[Grothendieck 1968] A. Grothendieck, Cohomologie locale des faisceaux cohérents et théorèmes de Lefschetz locaux et globaux (SGA 2), Advanced Studies in Pure Mathematics 2, North-Holland Publishing Co., Amsterdam, 1968. MR 57 \#16294 Zbl 0159.50402 
[Hartshorne 1966] R. Hartshorne, Residues and duality, Lecture Notes in Mathematics 20, Springer, Berlin, 1966. MR 36 \#5145 Zbl 0212.26101

[Hübl and Seibert 1997] R. Hübl and G. Seibert, "The adjunction morphism for regular differential forms and relative duality", Compositio Math. 106 (1997), 87-123. MR 98i:14023 Zbl 0886.14003

[Kempf et al. 1973] G. Kempf, F. F. Knudsen, D. Mumford, and B. Saint-Donat, Toroidal embeddings, I, Lecture Notes in Mathematics 339, Springer, Berlin, 1973. MR 49 \#299 Zbl 0271.14017

[Kollár 1986] J. Kollár, "Higher direct images of dualizing sheaves, I", Ann. of Math. (2) 123:1 (1986), 11-42. MR 87c:14038 Zbl 0598.14015

[Kollár and Mori 1998] J. Kollár and S. Mori, Birational geometry of algebraic varieties, Cambridge Tracts in Mathematics 134, Cambridge University Press, 1998. MR 2000b:14018 Zbl 0926.14003

[Kunz 1986] E. Kunz, Kähler differentials, Vieweg \& Sohn, Braunschweig, 1986. MR 88e:14025 Zbl 0587.13014

[Lipman 1984] J. Lipman, Dualizing sheaves, differentials and residues on algebraic varieties, Astérisque 117, Société Mathématique de France, Paris, 1984. MR 86g:14008 Zbl 0562.14003

[Lipman and Hashimoto 2009] J. Lipman and M. Hashimoto, Foundations of Grothendieck duality for diagrams of schemes, Lecture Notes in Mathematics 1960, Springer, Berlin, 2009. MR 2010b: $18001 \mathrm{Zbl} 1163.14001$

[Mac Lane 1998] S. Mac Lane, Categories for the working mathematician, 2nd ed., Graduate Texts in Mathematics 5, Springer, New York, 1998. MR 2001j:18001 Zbl 0906.18001

[Viehweg 1977] E. Viehweg, "Rational singularities of higher dimensional schemes", Proc. Amer. Math. Soc. 63:1 (1977), 6-8. MR 55 \#5624 Zbl 0352.14003

Communicated by János Kollár

Received 2009-12-02 Revised 2011-01-17 Accepted 2011-03-01

a.chatzistamatiou@uni-due.de Fachbereich Mathematik, Campus Essen, University of Duisburg-Essen, D-45117 Essen, Germany

kay.ruelling@uni-due.de Fachbereich Mathematik, Campus Essen, University of Duisburg-Essen, D-45117 Essen, Germany 


\title{
Geometry of quiver Grassmannians of Kronecker type and applications to cluster algebras
}

\author{
Giovanni Cerulli Irelli and Francesco Esposito
}

\begin{abstract}
We study quiver Grassmannians associated with indecomposable representations (of finite dimension) of the Kronecker quiver. We find a cellular decomposition for them and we compute their Betti numbers. As an application, we find a geometric realization for the atomic basis of cluster algebras of type $A_{1}^{(1)}$ found by Sherman and Zelevinsky (who called it the canonical basis) and those of type $A_{2}^{(1)}$ found in an earlier paper of the first author.
\end{abstract}

\section{Introduction}

Cluster algebras are commutative $\mathbb{Z}$-subalgebras of the field of rational functions in a finite number of indeterminates which have been introduced and studied in [Fomin and Zelevinsky 2002; 2003a; 2007; Berenstein et al. 2005]. To every quiver $Q$ without loops and 2-cycles it is associated a coefficient-free cluster algebra $\mathscr{A}_{Q}$. In [Caldero and Chapoton 2006; Caldero and Keller 2006; 2008; Derksen et al. 2010] the authors describe the cluster variables of $\mathscr{A}_{Q}$ via a map, called the CalderoChapoton map, between the representations of $Q$ and the field of rational functions in $n$ variables (we address the reader to the survey [Keller 2010]). This map is defined in terms of the Euler-Poincaré characteristic of some complex projective varieties attached to every finite-dimensional representation $M$ of $Q$ and called quiver Grassmannians. By definition, the quiver $\operatorname{Grassmannian} \mathrm{Gr}_{\boldsymbol{e}}(M)$ consists of all subrepresentations of $M$ of dimension vector $\boldsymbol{e}$. These varieties have been considered in, for example, [Caldero and Keller 2008; Caldero and Keller 2006; Caldero and Reineke 2008; Hernandez and Leclerc 2010, §12.3; Nakajima 2011]. In this paper we obtain more geometric information about them in the case of the Kronecker quiver. In [Caldero and Zelevinsky 2006] the authors compute the EulerPoincare characteristic of quiver Grassmannians associated with the Kronecker

Research supported by grant CPDA071244/07 of Padova University.

MSC2000: primary 06B15; secondary 16G20, 14N05, 13F99, 16G99, 05E10.

Keywords: complex algebraic geometry, quiver Grassmannians, cluster algebras, quiver representations. 
quiver and they conjecture the existence of a cellular decomposition which we find here. In [Cerulli Irelli 2011b] (see also [Poettering 2010]) a torus action on some quiver Grassmannians has been found and this allows to produce a cellular decomposition of them in the case they are smooth.

In order to get more concrete results, such as an explicit computation of Poincaré polynomials, we restrict our attention to the case of the Kronecker quiver. So in this paper we study quiver Grassmannians associated with finite-dimensional representations of the Kronecker quiver. A finite-dimensional representation of the Kronecker quiver (called from now on a $Q$-representation) is a quadruple $M=\left(M_{1}, M_{2}, m_{a}, m_{b}\right)$, where $M_{1}$ and $M_{2}$ are finite-dimensional complex vector spaces and $m_{a}, m_{b}: M_{1} \rightarrow M_{2}$ are two linear maps between them. Given two nonnegative integers $e_{1}$ and $e_{2}$, the variety $\operatorname{Gr}_{\left(e_{1}, e_{2}\right)}(M)$ is defined as the set

$$
\left\{\left(N_{1}, N_{2}\right) \in \mathrm{Gr}_{e_{1}}\left(M_{1}\right) \times \mathrm{Gr}_{e_{2}}\left(M_{2}\right): m_{a}\left(N_{1}\right) \subset N_{2}, m_{b}\left(N_{1}\right) \subset N_{2}\right\},
$$

where $\mathrm{Gr}_{e}(V)$ denotes the Grassmannian of $e$-dimensional vector spaces in a vector space $V$. This is a projective variety which is in general not smooth. When $M_{1}=$ $M_{2}=\mathbb{C}^{n}$ and $m_{a}=\mathrm{Id}$ is the identity matrix and $m_{b}=J_{n}(0)$ is an indecomposable nilpotent Jordan block, the representation $M$ is regular indecomposable and we denote it by $R_{n}$. The corresponding quiver Grassmannians $X=\operatorname{Gr}_{\left(e_{1}, e_{2}\right)}\left(R_{n}\right)$ are the main subjects of this paper. We concentrate on $R_{n}$ because it is an indecomposable (finite-dimensional) nonrigid $Q$-representation; all the other indecomposable (finitedimensional) $Q$-representations have either the same quiver Grassmannians as $R_{n}$ or they are rigid and hence their quiver Grassmannians are smooth and our results are already known for them (see Section 2.1). In Section 2.2 we find that a onedimensional torus $T$ acts on $X$. We provide a stratification of $X$ (see Section 2.5)

$$
X=X_{0} \supseteq X_{1} \supseteq \cdots \supseteq X_{s}
$$

for $s=\min \left(e_{1}, n-e_{2}\right)$ into closed subvarieties $X_{k} \simeq \operatorname{Gr}_{\left(e_{1}-k, e_{2}-k\right)}\left(R_{n-2 k}\right)$. Moreover $X_{k+1}$ is the singular locus of $X_{k}$ and the difference $X_{k} \backslash X_{k+1}$ is a smooth quasiprojective variety which is not complete. As a consequence of the stratification (1) we get that $X$ is smooth if and only if $s=0$, i.e., either $e_{1}=0$ or $e_{2}=n$, in which cases the quiver Grassmannian specializes to an usual Grassmannian of vector subspaces.

In Section 2.6 we prove that Białynicki-Birula's theorem on cellular decomposition of smooth projective varieties applies to $X_{k} \backslash X_{k+1}$; hence we can prove that $X$ has a decomposition

into attracting sets

$$
X=\bigcup_{L \in X^{T}} X_{L}
$$

$$
X_{L}:=\left\{N \in X: \lim _{\lambda \rightarrow 0} t_{\lambda} N=L\right\}
$$


of $T$-fixed points $L$, and theses sets are affine spaces. In Section 2.7 we describe the cell $X_{L}$ : if $L$ is indecomposable then $X_{L}$ is the orbit of $L$ by the action of a unipotent group; if $L=L^{\prime} \oplus L^{\prime \prime}$, with $L^{\prime}$ of "lower weight" than $L^{\prime \prime}$, we have

$$
\operatorname{dim} X_{L^{\prime} \oplus L^{\prime \prime}}=\operatorname{dim} X_{L^{\prime}}+\operatorname{dim} X_{L^{\prime}}-\left\langle\operatorname{dim} L^{\prime}, \operatorname{dim} L^{\prime \prime}\right\rangle,
$$

where $\langle\cdot, \cdot\rangle$ denotes the Euler form associated with the Kronecker quiver. As a consequence of this formula we are able to compute the Poincaré polynomials of the quiver Grassmannians associated with every finite-dimensional indecomposable representation of the Kronecker quiver: for $n \geq 0$ let $P_{n}$ (resp. $I_{n}$ ) be the indecomposable preprojective of dimension vector $(n, n+1)($ resp. $(n+1, n))$. As usual we denote by $P_{X}(t):=\sum_{i} \operatorname{dim} H^{i}(X) t^{i}$ the Poincaré polynomial of the complex projective variety $X$. Denoting by $\operatorname{Gr}_{t}(s)$ the Grassmannian of $t$-dimensional vector subspaces of an $s$-dimensional vector space, we have (see Section 2.8)

$$
\begin{aligned}
P_{\mathrm{Gr}_{e}\left(R_{n}\right)}(t) & =P_{\mathrm{Gr}_{\left(e_{2}-e_{1}\right)}\left(e_{2}\right)}(t) P_{\mathrm{Gr}_{\left(e_{2}-e_{1}\right)}\left(n-e_{1}\right)}(t), \\
P_{\mathrm{Gr}_{e}\left(P_{n}\right)}(t) & =P_{\mathrm{Gr}_{e_{1}}\left(e_{2}-1\right)}(t) P_{\mathrm{Gr}_{\left(e_{2}-e_{1}\right)}\left(n+1-e_{1}\right)}(t), \\
P_{\mathrm{Gr}_{e}\left(I_{n}\right)}(t) & =P_{\mathrm{Gr}_{e_{1}}\left(e_{2}+1\right)}(t) P_{\mathrm{Gr}_{\left(e_{2}-e_{1}\right)}\left(n-e_{1}\right)}(t),
\end{aligned}
$$

where $\boldsymbol{e}:=\left(e_{1}, e_{2}\right)$ denotes a dimension vector.

Szanto [2011] computed the polynomials which count the number of $\mathbb{F}_{q}$-points of the quiver Grassmannians associated with indecomposable (finite-dimensional) $Q$-representations. The polynomials he finds are precisely the ones we find: the Poincaré polynomials. This result is expected and should follow by standard technique in $\ell$-adic cohomology of schemes. It would deserve to be analyzed in more detail. In particular it would be interesting to know if the cellular decomposition continues to hold in positive characteristic.

In Section 3 we consider the (coefficient-free) cluster algebra $\mathscr{A}$ of type $A_{1}^{(1)}$. The cluster variables of $\mathscr{A}$ are the rational functions $x_{m}, m \in \mathbb{Z}$, of the field $\mathscr{F}=\mathbb{Q}\left(x_{1}, x_{2}\right)$ recursively generated by the relation

$$
x_{k} x_{k+2}=x_{k+1}^{2}+1
$$

It is not hard to see that every pair $\left\{x_{k}, x_{k+1}\right\}$ of consecutive cluster variables is a free-generating system for the field $\mathscr{F}$ and hence $\mathscr{F}_{F}=\mathbb{Q}\left(x_{k}, x_{k+1}\right)$ and every cluster variable can be expressed as a rational function in every such pair (which are called the clusters of $\mathscr{A}$ ). Every cluster variable is a Laurent polynomial in every cluster of $A$; this is the Laurent phenomenon proved in [Fomin and Zelevinsky 2002]. In this case the Caldero-Chapoton map $M \mapsto C C(M)$ associates to a $Q$-representation $M$ of dimension vector $\left(d_{1}, d_{2}\right)$ the Laurent polynomial 


$$
C C(M):=\frac{\sum_{\boldsymbol{e}} \chi\left(\mathrm{Gr}_{\boldsymbol{e}}(M)\right) x_{1}^{2\left(d_{2}-e_{2}\right)} x_{2}^{2 e_{1}}}{x_{1}^{d_{1}} x_{2}^{d_{2}}}
$$

Caldero and Keller [2008] proved that the map $M \mapsto C C(M)$ restricts to a bijection between the indecomposable rigid $Q$-representations $M$ (i.e., $\operatorname{Ext}^{1}(M, M)=0$ ) and the cluster variables of $\mathscr{A}$ different from $x_{1}$ and $x_{2}$. Moreover it has the property that $C C(M \oplus N)=C C(M) C C(N)$, under which cluster monomials not divisible by $x_{1}$ or $x_{2}$, i.e., monomials of the form $x_{k}^{a} x_{k+1}^{b}$ for $k \in \mathbb{Z} \backslash\{1,2\}$ and $a, b \geq 0$, are in bijection with rigid $Q$-representations.

Sherman and Zelevinsky [2004] introduced distinguished elements $\left\{z_{n}: n \geq 0\right\}$ of $\mathscr{F}$ recursively defined by

$$
z_{0}=2, \quad z_{1}=x_{0} x_{3}-x_{1} x_{2}, \quad z_{n+1}=z_{1} z_{n}-z_{n-1} \quad \text { for } n \geq 1,
$$

and proved that the set

$$
\boldsymbol{B}:=\{\text { cluster monomials }\} \cup\left\{z_{n}: n \geq 1\right\}
$$

is a $\mathbb{Z}$-basis of $\mathscr{A}$ such that positive linear combinations of its elements coincide with the set of all positive elements of $\mathscr{A}$, that is, elements that are positive Laurent polynomials in every cluster of $\mathscr{A}$. Such a basis is now called an atomic basis of $\mathscr{A}$, though Sherman and Zelevinsky called it a canonical basis. The terminology changed after the relationship between this basis and Lusztig's canonical basis was better understood; see [Lampe 2011; Geiß et al. 2012]. We give a new geometric realization of $\boldsymbol{B}$ by using the Caldero-Chapoton map: cluster monomials are images of rigid representations and quiver Grassmannians associated with rigid quiver representations are smooth [Caldero and Reineke 2008]. With this in mind we prove in Section 3 that

$$
z_{n}=\frac{\sum_{\boldsymbol{e}} \chi\left(\mathrm{Gr}_{\boldsymbol{e}}\left(R_{n}\right)^{\mathrm{sm}}\right) x_{1}^{2\left(n-e_{2}\right)} x_{2}^{2 e_{1}}}{x_{1}^{n} x_{2}^{n}}
$$

for every $n \geq 1$, where $\operatorname{Gr}_{e}\left(R_{n}\right)^{\mathrm{sm}}:=X_{0} \backslash X_{1}$ denotes the smooth part of $\operatorname{Gr}_{e}\left(R_{n}\right)$.

A similar construction can be made in a cluster algebra $\mathscr{A}_{2}$ of type $A_{2}^{(1)}$. These cluster algebras are studied in [Cerulli Irelli 2009] and some results are recalled in Section 3.2: the atomic basis of $\mathscr{A}_{2}$ consists of cluster monomials together with elements $\left\{u_{n} w^{k}, u_{n} z^{k}: k \geq 0, n \geq 1\right\}$ where $w$ and $z$ are two cluster variables and the $u_{n}$ are defined similarly to (4) as follows:

$$
u_{0}=2, \quad u_{1}=z w-2, \quad u_{n+1}=u_{1} u_{n}-u_{n-1} \quad \text { for } n \geq 1 .
$$

We prove that $u_{n}$ is obtained by evaluating the Caldero-Chapoton map at the smooth part of the regular indecomposable representation of a quiver of type $A_{2}^{(1)}$ of dimension vector $(n, n, n)$ that lies in a homogeneous tube. 


\section{Geometric structure of quiver Grassmannians}

Let

$$
Q:=1 \stackrel{a}{\stackrel{b}{\longrightarrow}} 2
$$

be the Kronecker quiver. As usual, we denote a complex $Q$-representation $M=$ $\left(M_{1}, M_{2}, m_{a}, m_{b}\right)$ as follows:

$$
M=M_{1} \stackrel{m_{a}}{\stackrel{m_{b}}{\longrightarrow}} M_{2} .
$$

A subrepresentation $N$ of $M$ consists of vector subspaces $N_{1}$ and $N_{2}$ of $M_{1}$ and $M_{2}$ respectively such that $m_{a}\left(N_{1}\right) \subset N_{2}$ and $m_{b}\left(N_{1}\right) \subset N_{2}$. We call

$$
\operatorname{dim}(M):=\left(\operatorname{dim} M_{1}, \operatorname{dim} M_{2}\right)
$$

the dimension vector of $M$. A morphism $g: M \rightarrow M^{\prime}$ from a $Q$-representation $M$ to a $Q$-representation $M^{\prime}$ is a pair $\left(g_{1}, g_{2}\right)$ of linear maps $g_{1}: M_{1} \rightarrow M_{1}^{\prime}$ and $g_{2}: M_{2} \rightarrow M_{2}^{\prime}$ such that $m_{a}^{\prime} \circ g_{1}=g_{2} \circ m_{a}$ and $m_{b}^{\prime} \circ g_{1}=g_{2} \circ m_{b}$. The set of $Q$-representations form a category which is an abelian Krull-Schmidt category via the natural notions of direct sums, kernel and cokernel (see [Assem et al. 2006], for example). The classification of finite-dimensional $Q$-representations which are indecomposable - that is, not direct sums of nontrivial subrepresentations - goes back to [Kronecker 1890]. Here is their complete list.

There are the indecomposable preprojectives

$$
P_{n}=k^{n} \underset{\varphi_{2}}{\stackrel{\varphi_{1}}{\longrightarrow}} k^{n+1},
$$

for $n \geq 0$, where $k=\mathbb{C}$ denotes the field of complex numbers, $k^{n}$ and $k^{n+1}$ denote complex vector spaces endowed respectively with linear bases $\left\{v_{1}^{(1)}, \ldots, v_{n}^{(1)}\right\}$ and $\left\{v_{1}^{(2)}, \ldots, v_{n+1}^{(2)}\right\}$, and $\varphi_{1}, \varphi_{2}$ are the immersions in the vector subspace of $k^{n+1}$ spanned by $\left\{v_{1}^{(2)}, \ldots, v_{n}^{(2)}\right\}$ and $\left\{v_{2}^{(2)}, \ldots, v_{n+1}^{(2)}\right\}$, respectively.

There are the indecomposable regulars

$$
R_{n}(\lambda)=k^{n} \underset{J_{n}(\lambda)}{\stackrel{\mathrm{Id}}{\longrightarrow}} k^{n}, \quad R_{n}(\infty)=k^{n} \underset{\mathrm{Id}}{\stackrel{J_{n}(0)}{\longrightarrow}} k^{n},
$$

where $\lambda \in k, J_{n}(\lambda)$ denotes the $n$-th indecomposable Jordan block of eigenvalue $\lambda$ and Id is the identity matrix (in the introduction we used the notation $R_{n}:=R_{n}(0)$ ).

Finally, there are the indecomposable preinjectives

$$
I_{n}=k^{n+1} \underset{\varphi_{1}^{t}}{\stackrel{\varphi_{2}^{t}}{\longrightarrow}} k^{n}
$$

for $n \geq 0$, where $\varphi_{1}^{t}$ and $\varphi_{2}^{t}$ are transposes of the matrices $\varphi_{1}$ and $\varphi_{2}$ defined above. 
For an indecomposable $Q$-representation $M$ we denote by $\mathscr{B}_{i}=\left\{v_{k}^{(i)}\right\}$, where $i=1,2$, the basis of $M_{i}$ with respect to which $M$ has the previous presentation. All other finite-dimensional $Q$-representations are direct sums of these ones. Direct sums of indecomposable preprojectives (resp. regulars, preinjectives) are called preprojective (resp. regular, preinjective) $Q$-representations.

Given nonnegative integers $e_{1}$ and $e_{2}$ and a $Q$-representation $M$ we consider the variety

$$
\operatorname{Gr}_{e}(M):=\left\{N \leq Q M: \operatorname{dim}(N)=\left(e_{1}, e_{2}\right)\right\},
$$

called the $\boldsymbol{e}=\left(e_{1}, e_{2}\right)$-quiver Grassmannian of $M$ (here $N \leq{ }_{Q} M$ means that $N$ is a subrepresentation of $M)$. This is closed inside the product $\mathrm{Gr}_{e_{1}}\left(M_{1}\right) \times \mathrm{Gr}_{e_{2}}\left(M_{2}\right)$ of usual Grassmannians of vector subspaces and so is a complex projective variety. In [Caldero and Reineke 2008] it is shown that the tangent space $T_{N}\left(\mathrm{Gr}_{e}(M)\right)$ at a point $N$ of $\operatorname{Gr}_{e}(M)$ equals:

$$
T_{N}\left(\operatorname{Gr}_{\boldsymbol{e}}(M)\right)=\operatorname{Hom}(N, M / N) .
$$

The following inequalities hold for $Z:=\mathrm{Gr}_{e}(M)$ :

$$
\langle\boldsymbol{e}, \boldsymbol{d}-\boldsymbol{e}\rangle \leq \operatorname{dim} Z \leq \operatorname{dim} T_{N}(Z) \leq\langle\boldsymbol{e}, \boldsymbol{d}-\boldsymbol{e}\rangle+\operatorname{dim} \operatorname{Ext}^{1}(M, M),
$$

where $\left\langle(a, b)^{t},(c, d)^{t}\right\rangle:=a c+b d-2 a d$ is the Euler form of $Q$ and $\boldsymbol{d}:=\operatorname{dim} M$, so that $\operatorname{dim}(M / N)=\boldsymbol{d}-\boldsymbol{e}$. In particular, if $M$ is $\operatorname{rigid}\left(\operatorname{Ext}^{1}(M, M)=0\right)$, then all the quiver Grassmannians associated with it are smooth (see [Derksen et al. 2010, Proposition 3.5] for a generalization of this result) and they have dimension $\operatorname{dim} \operatorname{Gr}_{\boldsymbol{e}}(M)=\langle\boldsymbol{e}, \boldsymbol{d}-\boldsymbol{e}\rangle$. It is known that the rigid $Q$-representations are

$$
P_{n}^{\oplus a} \oplus P_{n+1}^{\oplus b}, \quad I_{n}^{\oplus a} \oplus I_{n+1}^{\oplus b}
$$

for all $n \geq 0$ and $a, b \geq 0$.

2.1. Action of a group on quiver Grassmannians. Let $M$ be a $Q$-representation of dimension vector $\boldsymbol{d}=\left(d_{1}, d_{2}\right)$. We consider the group

$\mathcal{N}(M):=\left\{(A, B) \in \prod_{i=1}^{2} \mathrm{GL}_{d_{i}}\left(M_{i}\right): m_{a} A=B m_{a}, m_{b} A=\lambda B m_{b}\right.$ for some $\left.\lambda \in \mathbb{C}^{*}\right\}$.

Note that the automorphism group of $M$ is a closed subgroup of $\mathcal{N}(M)$ (for $\lambda=1)$. The group $\mathcal{N}(M)$ acts on $\operatorname{Gr}_{\boldsymbol{e}}(M)$ as follows $(A, B) \cdot\left(N_{1}, N_{2}\right):=\left(A N_{1}, B N_{2}\right)$.

Lemma 1. (1) For every $n \geq 0, \mathcal{N}\left(P_{n}\right) \simeq \mathbb{C}^{*} \times \mathbb{C}^{*}$.

(2) For every $n \geq 0, \mathcal{N}\left(I_{n}\right) \simeq \mathbb{C}^{*} \times \mathbb{C}^{*}$.

Proof. It follows easily from the definition that $\mathcal{N}\left(P_{n}\right)$ consists of diagonal matrices $(A, B)$ of the form

$$
A=\operatorname{diag}\left(a, a \lambda, \ldots, a \lambda^{n-1}\right), \quad B=\operatorname{diag}\left(a, a \lambda, \ldots, a \lambda^{n}\right)
$$


for $a, \lambda \in \mathbb{C}^{*}$. Similarly for $\mathcal{N}\left(I_{n}\right)$.

Proposition 2. Let $M$ be a rigid $Q$-representation. Then every quiver Grassmannian $\mathrm{Gr}_{\boldsymbol{e}}(M)$ associated with $M$ has a cellular decomposition.

Proof. The variety $\operatorname{Gr}_{e}(M)$ is smooth and $\mathcal{N}(M) \supset \mathbb{C}^{*}=: T$. The torus $T$ acts with finitely many fixed points. It follows hence by Białynicki-Birula results [BiałynickiBirula 1973] (see also [Chriss and Ginzburg 1997, §2.4]) that it has a cellular decomposition into attracting sets of its $T$-fixed points.

In the rest of the paper we mainly concentrate on the quiver Grassmannians associated with indecomposable regular $Q$-representations.

2.2. The variety $X=\mathbf{G r}_{e}\left(\boldsymbol{R}_{n}\right)$. From now on we will focus on quiver Grassmannians associated with indecomposable regular $Q$-representations. It is not hard to show that $\operatorname{Gr}_{e}\left(R_{n}(\lambda)\right)=\mathrm{Gr}_{e}\left(R_{n}(\mu)\right)$ for every $\lambda, \mu \in k \cup\{\infty\}$ (see [Cerulli Irelli 2011b], for example) and hence we consider the variety $X:=\operatorname{Gr}_{e}\left(R_{n}\right)$ (recall that our convention is $\left.R_{n}:=R_{n}(0)\right)$. It follows from the definition that

$$
X=\left\{N_{1} \subset N_{2} \subset k^{n}: J_{n}(0) N_{1} \subset N_{2}, \operatorname{dim} N_{i}=e_{i}, i=1,2\right\}
$$

and hence $X$ is a closed subvariety of a partial flag variety. In this paper we use the convention that

$$
J=J_{n}(0) \text { is a lower triangular matrix. }
$$

The group $\mathcal{N}:=\mathcal{N}\left(R_{n}\right)$ is given by

$$
\mathcal{N}:=\left\{A \in \mathrm{GL}_{n}(\mathbb{C}): A J A^{-1}=\lambda J, \text { for some } \lambda \in \mathbb{C}^{*}\right\},
$$

where $J:=J_{n}(0)$ and it acts on $X$ as $A \cdot\left(N_{1}, N_{2}\right)=\left(A N_{1}, A N_{2}\right)$.

Lemma 3. The group $\mathcal{N}$ is the semidirect product

$$
\mathcal{N}=U \rtimes T \times Z,
$$

where $\mathcal{U}$ is the unipotent radical of $\mathcal{N}$ of unipotent lower triangular Toeplitz matrices, given by

$$
u:=\left\{\mathbf{1}_{n}+\sum_{i=1}^{n-1} a_{i} J_{n}^{i}(0): a_{i} \in \mathbb{C}\right\},
$$

$T$ is the one-dimensional torus $T=\left\{t_{\lambda}: \lambda \in \mathbb{C}^{*}\right\}$, where $t_{\lambda}$ is the diagonal matrix $t_{\lambda}:=\operatorname{diag}\left(1, \lambda, \lambda^{2}, \ldots, \lambda^{n-1}\right)$, and $Z$ consists of central elements $\left\{a_{0} \mathbf{1}_{n}: a_{0} \in \mathbb{C}^{*}\right\}$.

Proof. Every element of $\mathcal{U} T Z$ belongs to $\mathcal{N}$. Conversely, let $A \in \mathcal{N}$. Then it is easy to see that the columns $\boldsymbol{a}_{1}, \ldots, \boldsymbol{a}_{n}$ of $A$ satisfy the relation $\boldsymbol{a}_{k+1}=J \boldsymbol{a}_{k}$ and hence $A \in U T Z$. It is now easy to see that $U$ is normal in $U T Z$. 
For example for $n=5$, an element $A$ of the group $U$ and an element $t_{\lambda}$ of $T$ have the form

$$
A=\left(\begin{array}{ccccc}
1 & 0 & 0 & 0 & 0 \\
a_{1} & 1 & 0 & 0 & 0 \\
a_{2} & a_{1} & 1 & 0 & 0 \\
a_{3} & a_{2} & a_{1} & 1 & 0 \\
a_{4} & a_{3} & a_{2} & a_{1} & 1
\end{array}\right) t_{\lambda}=\left(\begin{array}{ccccc}
1 & 0 & 0 & 0 & 0 \\
0 & \lambda & 0 & 0 & 0 \\
0 & 0 & \lambda^{2} & 0 & 0 \\
0 & 0 & 0 & \lambda^{3} & 0 \\
0 & 0 & 0 & 0 & \lambda^{4}
\end{array}\right)
$$

for $a_{1}, a_{2}, a_{3}, a_{4} \in \mathbb{C}$ and $\lambda \in \mathbb{C}^{*}$.

Let $X^{T}:=\{N \in X: t N=N$ for all $t \in T\}$. Clearly $L \in X^{T}$ if and only if $L$ is a coordinate subrepresentation of $R_{n}$, i.e., both $L_{1}$ and $L_{2}$ are coordinate subspaces of $k^{n}$. In the next section we will encode this information in a combinatorial tool which is called the coefficient quiver of $R_{n}$.

We conclude this section by pointing out the useful isomorphism

$$
\varphi_{n}: \mathrm{Gr}_{\boldsymbol{e}} \rightarrow \mathrm{Gr}_{e^{*}}\left(R_{n}\right), \quad \varphi_{n}(N)=N^{0},
$$

where $\left(e_{1}, e_{2}\right)^{*}:=\left(n-e_{2}, n-e_{1}\right)$ and $N^{0}:=\left\{f \in R_{n}^{*}: f(v)=0\right.$ for all $\left.v \in N\right\}$ by using the identification $R_{n} \simeq R_{n}^{*}$. The torus $T$ acts on $R_{n}^{*}$ with contragredient action and this gives an action on $\operatorname{Gr}_{e^{*}}\left(R_{n}^{*}\right)$. Under the identification $R_{n} \simeq R_{n}^{*}$ these two actions differ by a character and so the identification $\operatorname{Gr}_{e}\left(R_{n}^{*}\right) \simeq \operatorname{Gr}_{e}\left(R_{n}\right)$ is $T$-equivariant. It is an easy check that isomorphism (10) is $T$-equivariant and involutive.

2.3. The coefficient quiver of $\boldsymbol{R}_{\boldsymbol{n}}$. Following [Ringel 1998] we associate to a $Q$ representation $M$ with linear basis $\mathscr{B}$ a quiver $\Gamma(M, \mathscr{B})$ called the coefficient quiver of $M$ in the basis $\mathscr{B}$. By definition, $\Gamma(M, \mathscr{B})$ has the elements of $\mathscr{B}$ as vertices and there is an arrow labeled by $a$ (resp. $b$ ) between two vertices $v$ and $v^{\prime}$ if the coefficient of $v^{\prime}$ in $m_{a}(v)$ (resp. $m_{b}(v)$ ) is nonzero. We call $\Gamma(M, \mathscr{B})$ the coefficient quiver of $M$ in the basis $\mathscr{B}$. When the basis $\mathscr{B}$ of $M$ is clear from the definition of $M$ we simply write $\Gamma(M)$ for $\Gamma(M, \mathscr{B})$. Here are the coefficient quivers of some indecomposable $Q$-representations for $n=4$ in the basis defined in Section 2:

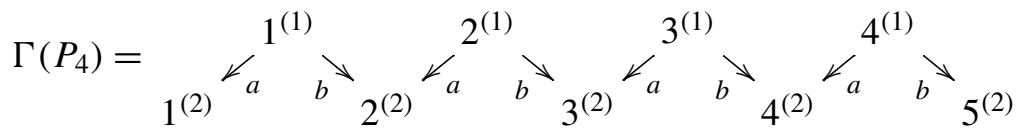

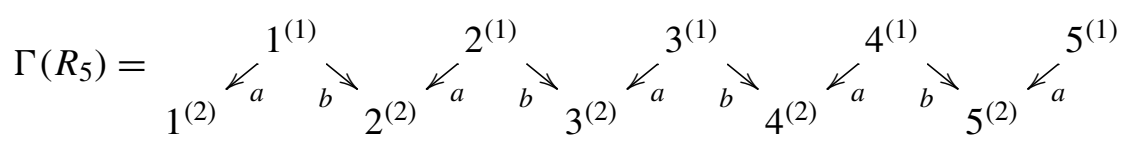

$$
\begin{aligned}
& \Gamma\left(I_{4}\right)=\quad 1^{(1)} \downarrow_{1^{(2)}} \swarrow_{a}^{2^{(1)}} b_{2^{(2)}} \swarrow_{a}^{3^{(1)}} b_{3^{(2)}}^{\downarrow_{a}}{ }_{b}^{4_{4}^{(2)}} \swarrow_{a}^{5^{(1)}}
\end{aligned}
$$


Here $k^{(i)}$, for $i=1,2$, denotes the vertex corresponding to $v_{k}^{(i)}$. Note that we use the convention (8).

In all these cases a one-dimensional torus $T$ acts on all the associated quiver Grassmannians and the fixed points of this action are in bijection with successor closed subquivers of the corresponding coefficient quiver (i.e., subquivers $\gamma$ such that if $v$ is a vertex of $\gamma$ and $\alpha: v \rightarrow v^{\prime}$ is an arrow with source $v$ then $\alpha$ is an arrow of $\gamma$ ).

Let us consider $\Gamma\left(R_{n}\right)$. In Lemma 3 we have seen that the torus $T$ acts on $X$ by $\lambda \cdot v_{k}^{(i)}=\lambda^{k-1} v_{k}^{(i)}(i=1,2, k \in[1, n])$. For every $r \geq 1$ there exists a unique regular subrepresentation of $R_{n}$ isomorphic to $R_{r}$ and it has the property that,

$$
\text { if } N \in X \text { is such that } \lim _{\lambda \rightarrow 0} t_{\lambda} N=R_{r} \text {, then } N=R_{r} \text {. }
$$

Indeed this subrepresentation is coordinate and lies in the extreme right-hand side of $\Gamma\left(R_{n}\right)$. In particular the basis elements that generate $R_{r}$ have maximal weights.

Roughly speaking, the flow for $\lambda \rightarrow 0$ goes from right to left in $\Gamma\left(R_{n}\right)$. For example the line $\left\langle v_{1}^{(2)}+v_{2}^{(2)}\right\rangle$ generated by the vector $v_{1}^{(2)}+v_{2}^{(2)}$ goes to the line generated by $v_{1}^{(2)}$ as follows:

$$
\lim _{\lambda \rightarrow 0} \lambda \cdot\left(\left\langle v_{1}^{(2)}+v_{2}^{(2)}\right\rangle\right)=\lim _{\lambda \rightarrow 0}\left(\left\langle v_{1}^{(2)}+\lambda v_{2}^{(2)}\right\rangle\right)=\left\langle v_{1}^{(2)}\right\rangle .
$$

2.4. Action of the torus on Hom-spaces. Let $L$ and $L^{\prime}$ be indecomposable $Q$ representations. As we have seen in the previous sections, the torus $T=\left\{t_{\lambda}\right.$ : $\left.\lambda \in \mathbb{C}^{*}\right\}$ acts on the quiver Grassmannians associated with them. The action of $T$ naturally extends to the vector space $\operatorname{Hom}\left(L, L^{\prime}\right)$ as follows: for $f \in \operatorname{Hom}\left(L, L^{\prime}\right)$, $\left(t_{\lambda} f\right)(l):=t_{\lambda} f\left(t_{\lambda^{-1}} l\right)$. Following [Crawley-Boevey 1989] we endow the vector space $\operatorname{Hom}\left(L, L^{\prime}\right)$ with a distinguished basis. Since $\operatorname{Hom}(\cdot, \cdot)$ is additive, we assume that both $L$ and $L^{\prime}$ are indecomposable. Let $\Gamma(L)$ and $\Gamma\left(L^{\prime}\right)$ denote the coefficient quiver of $L$ and $L^{\prime}$ respectively. We consider the set $\mathscr{G} \mathscr{B}\left(L, L^{\prime}\right)$ of triples $\left(\gamma, \alpha, \gamma^{\prime}\right)$ such that $\gamma$ is a connected predecessor closed subquiver of $\Gamma(L)$ (meaning that for every vertex $v$ of $\gamma$, every arrow $c: v^{\prime} \rightarrow v$ with target $v$ belongs to $\gamma), \gamma^{\prime}$ is a successor closed subquiver of $\Gamma\left(L^{\prime}\right)$, and $\alpha: \gamma_{0} \rightarrow \gamma_{0}^{\prime}$ is a bijection from the set $\gamma_{0}$ of vertices of $\gamma$ to the set $\gamma_{0}^{\prime}$ of vertices of $\gamma^{\prime}$, such that for every arrow

$$
v_{k}^{(1)} \stackrel{a}{\longrightarrow} v_{k}^{(2)}
$$

of $\gamma$ there is an arrow

$$
\alpha\left(v_{k}^{(1)}\right) \stackrel{a}{\longrightarrow} \alpha\left(v_{k}^{(2)}\right)
$$

of $\gamma^{\prime}$ with the same label, and likewise with $a$ replaced by $b$.

The next proposition is a special case of [Crawley-Boevey 1989], so we omit its proof (which is quite simple in this case). 
Proposition 4. Consider the map $B: \mathscr{C} \mathscr{B}\left(L, L^{\prime}\right) \rightarrow \operatorname{Hom}\left(L, L^{\prime}\right)$ that associates to $\left(\gamma, \alpha, \gamma^{\prime}\right)$ the homomorphism

$$
f_{\gamma \gamma^{\prime}}(v)=\left\{\begin{array}{cl}
\alpha(v) & \text { if } v \in \gamma_{0}, \\
0 & \text { otherwise. }
\end{array}\right.
$$

The image of $B$ is a basis of $\operatorname{Hom}\left(L, L^{\prime}\right)$ which we call $C B$-basis (for CrawleyBoevey).

The proposition is illustrated in the figure below, which shows an element of the $C B$-basis of $\operatorname{Hom}\left(R_{3}, R_{3}\right)$. The circles (resp. bullets) highlight a predecessor (successor) closed subquiver $\gamma$ (resp. $\gamma^{\prime}$ ) of $\Gamma\left(R_{3}\right)$. The dotted arrows show the corresponding $f_{\gamma \gamma^{\prime}}$.

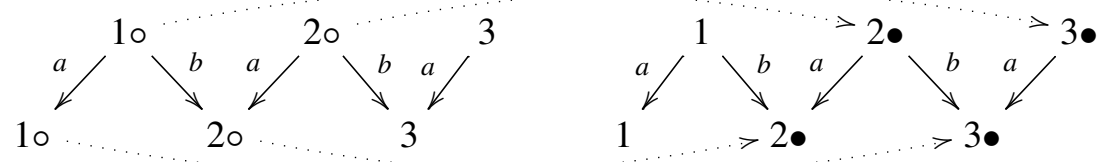

The torus $T$ acts diagonally on the elements of the $C B$-basis of $\operatorname{Hom}\left(L, L^{\prime}\right)$ as follows: If $\left(\gamma, \alpha, \gamma^{\prime}\right) \in \mathscr{C} \mathscr{B}\left(L, L^{\prime}\right)$ and the vertices of $\gamma$ have consecutive weights $k, k+1, \ldots$ and the vertices of $\gamma^{\prime}$ have consecutive weights $k^{\prime}, k^{\prime}+1, \ldots$ then

$$
t_{\lambda} f_{\gamma \gamma^{\prime}}=\lambda^{k^{\prime}-k} f_{\gamma \gamma^{\prime}}
$$

and we say that $f_{\gamma \gamma^{\prime}}$ has weight $k^{\prime}-k$. For example, the morphism illustrated above has weight 1 . We denote by $\operatorname{Hom}\left(L, L^{\prime}\right)^{+}$the vector subspace of $\operatorname{Hom}\left(L, L^{\prime}\right)$ spanned by $C B$-basis elements with positive weight.

As an application of Proposition 4 we compute the dimension of the Hom-spaces between indecomposable $Q$-representations. It is known (and not difficult to prove by using Proposition 4) that $\operatorname{Hom}\left(R_{s}, P_{l}\right)=\operatorname{Hom}\left(I_{s}, P_{l}\right)=\operatorname{Hom}\left(I_{s}, R_{l}\right)=0$ for all $s, l \geq 0$. Hence we consider the remaining cases.

Lemma 5. For every $l, s \geq 0$ we have

$$
\begin{aligned}
& \operatorname{dim} \operatorname{Hom}\left(P_{s}, P_{l}\right)=[l-s+1]_{+}, \\
& \operatorname{dim} \operatorname{Hom}\left(P_{s}, R_{l}\right)=l, \\
& \operatorname{dim} \operatorname{Hom}\left(P_{s}, I_{l}\right)=l+s, \\
& \operatorname{dim} \operatorname{Hom}\left(R_{s}, R_{l}\right)=\min (s, l), \\
& \operatorname{dim} \operatorname{Hom}\left(R_{s}, I_{l}\right)=s, \\
& \operatorname{dim} \operatorname{Hom}\left(I_{s}, I_{l}\right)=[s-l+1]_{+},
\end{aligned}
$$

where $[b]_{+}:=\max (b, 0)$. 
We conclude this section by pointing out that the action of the torus $T$ on $\operatorname{Hom}\left(L, L^{\prime}\right)$ induces an action of the torus on the space $\operatorname{Ext}^{1}\left(L, L^{\prime}\right)$. With respect to this action long exact sequences in cohomology are $T$-equivariant.

2.5. Stratification of $X$. Every subrepresentation $N$ of $R_{n}$ is of the form $N=$ $P \oplus R_{r}$ where $P$ is preprojective and $R_{r}$, for $r \geq 0$, is either zero or an indecomposable regular $Q$-representation. Similarly the quotient $R_{n} / N=R_{r^{\prime}} \oplus I$ where $R_{r^{\prime}}$, for $r^{\prime} \geq 0$, is either zero or regular indecomposable and $I$ is preinjective. This allows us to give the following definition.

Definition 6. Let $N \in X$ with $N=P \oplus R_{r}$ and $R_{n} / N=R_{r^{\prime}} \oplus I$ with $P$ preprojective, $I$ preinjective and some $r, r^{\prime} \geq 0$. We define the integer

$$
K_{N}=K_{N}(X):=\min \left(r, r^{\prime}\right)
$$

It is easy to see that $K_{N}=\operatorname{dim} \operatorname{Ext}^{1}\left(N, R_{n} / N\right)$. Indeed

$\operatorname{dim} \operatorname{Ext}^{1}\left(N, R_{n} / N\right)=\operatorname{dim} \operatorname{Ext}^{1}\left(R_{r}, R_{r^{\prime}}\right)=\operatorname{dim} \operatorname{Hom}\left(R_{r^{\prime}}, R_{r}\right)=\min \left(r, r^{\prime}\right)$

where in the first equality we use the well-known fact that

$$
\operatorname{Ext}^{1}(R, I)=\operatorname{Ext}^{1}(P, R)=\operatorname{Ext}^{1}(P, I)=0
$$

for every preprojective $P$, regular $R$ and preinjective $I Q$-representations; in the second equality we use the AR-formula (see [Assem et al. 2006], for example); in the last equality we use (15). In particular it is known that

$$
\left\langle\operatorname{dim} N, \operatorname{dim} N^{\prime}\right\rangle=\operatorname{dim} \operatorname{Hom}\left(N, N^{\prime}\right)-\operatorname{dim} \operatorname{Ext}^{1}\left(N, N^{\prime}\right)
$$

and in view of (6) we get that for every $N \in X$ the dimension of the tangent space $T_{N}(X)$ at $N$ equals

$$
\operatorname{dim} T_{N}(X)=\langle\boldsymbol{e}, n \delta-\boldsymbol{e}\rangle+K_{N} .
$$

where $\delta:=(1,1)^{t}$. Equation (18) implies that a point $N \in X$ is smooth if and only if either $N$ or $R_{n} / N$ do not have a regular direct summand.

The next theorem provides a stratification of $X$ and it is essential for our proof of the existence of a cellular decomposition of $X$. Let us define the strata.

Definition 7. For every integer $k \geq 0$ define the set

$$
X_{k}=X_{k}(X):=\left\{N \in X: K_{N} \geq k\right\}
$$

where $K_{N}$ is given in Definition 6.

Theorem 8. (1) The set $X_{k+1}$ is a closed T-stable subvariety of $X_{k}$. Moreover there is a $T$-equivariant isomorphism

$$
X_{k} \simeq \operatorname{Gr}_{\left(e_{1}-k, e_{2}-k\right)}\left(R_{n-2 k}\right) .
$$


(2) The subvarieties $X_{k}$ 's provide a stratification of $X$

$$
X=X_{0} \supset X_{1} \supset \cdots \supset X_{s}
$$

where $s=\min \left(e_{1}, n-e_{2}\right)$.

(3) The variety $X_{k} \backslash X_{k+1}$ is smooth (inside $X_{k}$ ) and

$$
\lim _{\lambda \rightarrow 0} t_{\lambda} N \in X_{k} \backslash X_{k+1} \quad \text { for all } N \in X_{k} \backslash X_{k+1} .
$$

Proof. We consider two subvarieties of $X$ :

$$
X_{k}^{\prime}(X):=\left\{N \in \operatorname{Gr}_{e}\left(R_{n}\right): R_{k} \text { is a subrepresentation of } N\right\}
$$

for $k \in\left[0, e_{1}\right]$, and

$$
X_{k}^{\prime \prime}(X):=\left\{N \in \operatorname{Gr}_{e}\left(R_{n}\right): R_{k} \text { is a quotient of } R_{n} / N\right\}
$$

for $k \in\left[0, n-e_{2}\right]$. It follows from the definitions that

$$
X_{k}=X_{k}^{\prime} \cap X_{k}^{\prime \prime} \text {. }
$$

We now collect some properties of $X_{k}^{\prime}$ and $X_{k}^{\prime \prime}$.

Lemma 9. (1) The isomorphism $\varphi_{n}$ defined in (10) induces an isomorphism

$$
X_{k}^{\prime}\left(\operatorname{Gr}_{e}\left(R_{n}\right)\right) \simeq X_{k}^{\prime \prime}\left(\operatorname{Gr}_{e^{*}}\left(R_{n}\right)\right),
$$

and hence also

$$
X_{k}^{\prime \prime}\left(\operatorname{Gr}_{e}\left(R_{n}\right)\right) \simeq X_{k}^{\prime}\left(\operatorname{Gr}_{e^{*}}\left(R_{n}\right)\right) .
$$

(2) For every $k \in\left[0, e_{1}\right], X_{k}^{\prime}(X)$ is a T-stable subvariety of $X$. For every $N \in$ $X_{k}^{\prime}(X), \lim _{\lambda \rightarrow 0} t_{\lambda} N \in X_{k}^{\prime}(X)$. There is a T-equivariant isomorphism

$$
X_{k}^{\prime}(X) \simeq \operatorname{Gr}_{e-k \delta}\left(R_{n-k}\right) .
$$

(3) For every $k \in\left[0, n-e_{2}\right], X_{k}^{\prime \prime}(X)$ is a $T$-stable subvariety of $X$. For every $N \in X_{k}^{\prime \prime}(X), \lim _{\lambda \rightarrow 0} t_{\lambda} N \in X_{k}^{\prime \prime}(X)$. There is a T-equivariant isomorphism

$$
X_{k}^{\prime \prime}(X) \simeq \operatorname{Gr}_{e}\left(R_{n-k}\right) .
$$

Proof. Part (1) is a straightforward check. Part (3) follows from part (1) by using the isomorphism (10). Part (2) follows from property (11) of $R_{k}$. It remains to check (24). We consider the map

$$
\Phi: X_{k}^{\prime}(X) \rightarrow \operatorname{Gr}_{e-k \delta}\left(R_{n-k}\right) \text { given by } \Phi(N)=\left(N / R_{k}\right) .
$$

Since there is a unique exact sequence of regular $Q$-representations

$$
0 \longrightarrow R_{k} \stackrel{\iota_{k}}{\longrightarrow} R_{n} \stackrel{\pi_{n-k}}{\longrightarrow} R_{n-k} \longrightarrow 0
$$

the map $\Phi$ is well defined and bijective. 
We now conclude the proof of Theorem 8. Everything follows from Lemma 9 by (23) except the smoothness of $X_{k} \backslash X_{k+1}$. To prove smoothness notice that it is sufficient to prove smoothness of $X_{0} \backslash X_{1}$ by (19), since $K_{N}\left(X_{k}\right)=K_{N}(X)-k$. By (18) $X_{0} \backslash X_{1}$ is the smooth locus of $X$.

Corollary 10. The variety $X=\operatorname{Gr}_{e}\left(R_{n}\right)$ is smooth if and only if $e_{1}=0$ (in which case $X \simeq \mathrm{Gr}_{e_{2}}\left(k^{n}\right)$ ) or $e_{2}=n$ (in which case $X \simeq \mathrm{Gr}_{e_{1}}\left(k^{n}\right)$ ).

Proof. By Theorem 8, $X$ is smooth if and only if $X=X_{0}$ if and only if $s=$ $\min \left(e_{1}, n-e_{2}\right)=0$.

Corollary 11. The quiver Grassmannian $\operatorname{Gr}_{\left(e_{1}, e_{2}\right)}\left(R_{n}\right)$ has dimension

$$
\operatorname{dim} \operatorname{Gr}_{\left(e_{1}, e_{2}\right)}\left(R_{n}\right)=\langle\boldsymbol{e}, n \delta-\boldsymbol{e}\rangle=\left(e_{2}-e_{1}\right)\left(n-\left(e_{2}-e_{1}\right)\right)
$$

Proof. In $X=\mathrm{Gr}_{e}\left(R_{n}\right)$ one can always find a smooth point, i.e., a subrepresentation $N$ of $R_{n}$ such that either $N$ or $R_{n} / N$ do not have a regular direct summand. It follows that $X_{0} \backslash X_{1}$ is nonempty and hence the result follows from (18).

2.6. Cellular decomposition of $X$. In this section we provide a cellular decomposition of $X=\operatorname{Gr}_{e}\left(R_{n}\right)$. Following [Białynicki-Birula 1973] (see also [Chriss and Ginzburg 1997]), for every fixed point $L \in X^{T}$ we consider its attracting set, defined as

$$
X_{L}:=\left\{N \in X: \lim _{\lambda \rightarrow 0} t_{\lambda} N=L\right\} .
$$

In particular, $L \in X_{L}$ for every $L \in X^{T}$.

Theorem 12. For every $L \in X^{T}$ the corresponding attracting set $X_{L}$ is an affine space and $X_{L} \simeq T_{L}\left(X_{L}\right) \simeq T_{L}^{+}(X):=\operatorname{Hom}\left(L, R_{n} / L\right)^{+}$. Moreover

$$
X=\bigcup_{L \in X^{T}} X_{L}
$$

Proof. The results from [Białynicki-Birula 1973] on cellular decomposition of a projective variety $X$ continue to hold if the variety is smooth but only quasiprojective, provided that the action of the torus is such that $\lim _{\lambda \rightarrow 0} t_{\lambda} N$ belongs to $X$ for every $N \in X$. This is an easy consequence of Hironaka's resolution of singularities. In view of Theorem 8 we apply this to $X_{0} \backslash X_{1}$ and we get the result.

2.7. Description of the cells. In this section we describe the cell $X_{L}$ associated with every $L \in X^{T}$ (see Theorem 12). Given integers $r \in[0, n-1]$ and $k \in[1, n-r]$, we denote by ${ }_{k}\left(P_{r}\right)$ the indecomposable preprojective subrepresentation of $R_{n}$ of dimension vector $(r, r+1)$ generated by $v_{k}^{(1)}, v_{k+1}^{(1)}, \ldots, v_{k+r-1}^{(1)}$ and $v_{k}^{(2)}, v_{k+1}^{(2)}, \ldots, v_{k+r}^{(2)}$ if $r \geq 1$ and by $v_{k}^{(2)}$ if $r=0$. For example, the following figure shows the subrepre- 
sentation $_{2}\left(P_{1}\right)$ of $R_{5}$ :

$1^{(2)}$
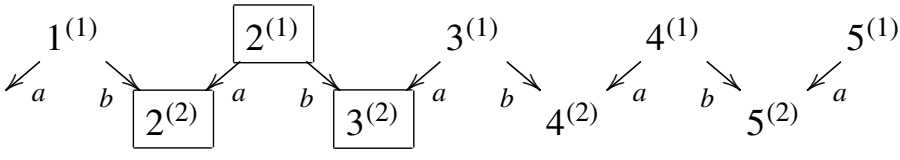

Theorem 13. (1) If $L \in X^{T}$ is indecomposable then $X_{L}=U L$. In particular if $L={ }_{k}\left(P_{e_{1}}\right)$ then $\operatorname{dim} X_{L}=n-k$.

(2) If $L=L^{\prime} \oplus L^{\prime \prime}$ with $\operatorname{Hom}\left(L^{\prime}, L^{\prime \prime}\right)^{+}=\operatorname{Hom}\left(L^{\prime}, L^{\prime \prime}\right)$ then

$$
\operatorname{dim} X_{L}=\operatorname{dim} X_{L^{\prime}}+\operatorname{dim} X_{L^{\prime \prime}}-\left\langle\operatorname{dim} L^{\prime}, \operatorname{dim} L^{\prime \prime}\right\rangle .
$$

Proof. (1) The unipotent group $U$ defined in Lemma 3 is a subgroup of dimension $n-1$ of the group of $n \times n$ unipotent lower triangular matrices. Let $L$ be an indecomposable subrepresentation of $R_{n}$ of dimension vector $\boldsymbol{e}$. If $L=R_{r}$ then $\boldsymbol{e}=(r, r)$ and $L_{1}=L_{2}$ is the vector subspace of $k^{n}$ spanned by the last $r$ basis vectors of $R_{n}$. In particular, $A L=L$ for every $A \in U$. On the other hand we have already noticed that regular subrepresentations have property (11) and hence $X_{L}=\{L\}$ and we get $X_{L}=U L$ if $L$ is regular. Let $L$ be an indecomposable preprojective subrepresentation of $R_{n}$. Then $e_{2}=e_{1}+1$ and we assume that $L={ }_{k}\left(P_{e_{1}}\right)$ for some $k \in\left[1, n-e_{1}\right]$. It is easy to see that $\operatorname{dim} U L=n-k$. Indeed the stabilizer of $L$ under the action of $U$ equals the stabilizer of the line generated by $v_{k}^{(2)}$ and hence it has dimension $k-1$. For example in (9) the stabilizer of the third basis vector is generated by $a_{3}$ and $a_{4}$ and has dimension two. We now prove that $\operatorname{dim} X_{L}=n-k$ and since $U L \subset X_{L}$ and they are both affine spaces, we get the equality. By Theorem 12 we have to compute $\operatorname{dim} T_{L}^{+}(X)=\operatorname{dim} \operatorname{Hom}\left(L, R_{n} / L\right)^{+}$. Since $L={ }_{k}\left(P_{e_{1}}\right)$ is indecomposable the quotient $R_{n} / L$ is the direct sum of at most two indecomposables as follows

$$
R_{n} / L=R_{k-1} \oplus I_{t}
$$

where $t=n-k-e_{1}$. We have

$$
\operatorname{Hom}\left(L, R_{n} / L\right)^{+}=\operatorname{Hom}\left(P_{e_{1}}, I_{t}\right) .
$$

In view of (14), $\operatorname{dim} \operatorname{Hom}\left(L, R_{n} / L\right)^{+}=e_{1}+t=n-k$ and we are done.

(2) There are short exact sequences

$$
0 \longrightarrow L^{\prime} \longrightarrow R_{n} / L^{\prime \prime} \longrightarrow R_{n} /\left(L^{\prime} \oplus L^{\prime \prime}\right) \longrightarrow 0
$$

and

$$
0 \longrightarrow L^{\prime \prime} \longrightarrow R_{n} / L^{\prime} \longrightarrow R_{n} /\left(L^{\prime} \oplus L^{\prime \prime}\right) \longrightarrow 0
$$


We apply the functors $\operatorname{Hom}\left(L^{\prime \prime},-\right)$ and $\operatorname{Hom}\left(L^{\prime},-\right)$ to the previous short exact sequences, then take the positive part (the part spanned by $C B$-basis elements with positive weight) and we get the exact sequences (see Theorem 12)

$$
0 \longrightarrow 0 \longrightarrow T_{L^{\prime \prime}}\left(X_{L^{\prime \prime}}\right) \longrightarrow \operatorname{Hom}\left(L^{\prime \prime}, R_{n} / L\right) \longrightarrow 0
$$

and

$$
0 \rightarrow \operatorname{Hom}\left(L^{\prime}, L^{\prime \prime}\right) \rightarrow T_{L^{\prime}}\left(X_{L^{\prime}}\right) \rightarrow \operatorname{Hom}\left(L^{\prime}, R_{n} / L\right) \rightarrow \operatorname{Ext}^{1}\left(L^{\prime}, L^{\prime \prime}\right)^{+} \rightarrow 0 .
$$

Indeed, we have

$$
\begin{aligned}
& \operatorname{Ext}^{1}\left(L^{\prime \prime}, L^{\prime}\right)^{+}=0=\operatorname{Ext}^{1}\left(L^{\prime}, R_{n} / L^{\prime}\right)^{+}, \\
& \operatorname{Ext}^{1}\left(L^{\prime}, L^{\prime \prime}\right)^{+}=\operatorname{Ext}^{1}\left(L^{\prime}, L^{\prime \prime}\right) .
\end{aligned}
$$

This is because, if $\operatorname{Hom}(M, L)^{+}=0$ then $\operatorname{Ext}^{1}(M, L)^{+}=0$; indeed one can always take a minimal $T$-equivariant injective resolution of $L$ and apply the functor $\operatorname{Hom}(M,-)$. By summing up we get the short exact sequence

$$
0 \rightarrow \operatorname{Hom}\left(L^{\prime}, L^{\prime \prime}\right) \rightarrow T_{L^{\prime}}\left(X_{L^{\prime}}\right) \oplus T_{L^{\prime \prime}}\left(X_{L^{\prime \prime}}\right) \rightarrow T_{L}\left(X_{L}\right) \rightarrow \operatorname{Ext}^{1}\left(L^{\prime}, L^{\prime \prime}\right) \rightarrow 0
$$

Since for every fixed point $L, \operatorname{dim} X_{L}=\operatorname{dim} T_{L}\left(X_{L}\right)$ we get (28).

2.8. Betti numbers. We now use the results of the previous sections in order to compute the Betti numbers of $X=\operatorname{Gr}_{e}\left(R_{n}\right)$. Since $X$ has a cellular decomposition (Theorem 12) the odd cohomology spaces of $X$ are zero and the $2 i$-th Betti number $b_{2 i}=b_{2 i}(X):=\operatorname{dim} H^{2 i}(X)$ equals the number of cells of dimension $i$.

Before stating the main result of this section we start with the special case $e_{2}=e_{1}+1$.

Theorem 14. Let $X=\operatorname{Gr}_{\left(e_{1}, e_{1}+1\right)}\left(R_{n}\right)$. The even Betti numbers of $X$ are

$$
b_{2 i}= \begin{cases}i+1 & \text { if } 0 \leq i \leq s \\ s+1 & \text { if } s \leq i \leq n-1-s \\ n-i & \text { if } n-1-s \leq i \leq n-1\end{cases}
$$

where $s=\min \left(e_{1}, n-1-e_{1}\right)($ see Theorem 8(2)).

The Poincaré polynomial $P_{X}(t):=\sum_{i=0}^{\operatorname{dim} X} b_{i} t^{i}$ of $X$ equals

$$
P_{X}\left(t^{1 / 2}\right)=\left(\frac{t^{e_{1}+1}-1}{t-1}\right)\left(\frac{t^{n-e_{1}}-1}{t-1}\right)
$$

Proof. For $k \in\left[0, e_{1}\right]$, consider the variety $X_{k}^{\prime}$ defined in (21) and the difference

$$
Y_{k}^{\prime}=Y_{k}^{\prime}(X):=X_{k}^{\prime}(X) \backslash X_{k+1}^{\prime}(X) \text {. }
$$


By definition, $N \in Y_{k}$ if $N=R_{k} \oplus P$ for some (indecomposable) preprojective $P$. Since $X$ is union of the $Y_{k}^{\prime}$ and each $Y_{k}^{\prime}$ is a union of cells (Theorem 12) we get

$$
\operatorname{dim} H^{i}(X)=\sum_{k=0}^{e_{1}} \operatorname{dim} H_{c}^{i}\left(Y_{k}^{\prime}\right),
$$

where $H_{c}^{i}\left(Y_{k}^{\prime}\right)$ is the $i$-th cohomology space of $Y_{k}^{\prime}$ with compact support and its dimension equals the number of cells of dimension $i / 2$. In particular $\operatorname{dim} H_{c}^{i}\left(Y_{k}^{\prime}\right)=0$ for $i$ odd. We prove that

$$
\operatorname{dim} H_{c}^{2 i}\left(Y_{k}^{\prime}\right)= \begin{cases}1 & \text { if } i \in\left[e_{1}-k, n-k-1\right], \\ 0 & \text { otherwise. }\end{cases}
$$

and hence (31) follows from (34). From the definition it follows that

$$
Y_{k}^{\prime}(X) \simeq Y_{0}^{\prime}\left(\operatorname{Gr}_{\left(e_{1}-k, e_{1}+1-k\right)}\left(R_{n-k}\right)\right) .
$$

The elements of $Y_{0}^{\prime}\left(\mathrm{Gr}_{\left(f_{1}, f_{1}+1\right)}\left(R_{m}\right)\right)$ are all the indecomposable preprojective subrepresentations of $R_{m}$ of dimension vector $\left(f_{1}, f_{1}+1\right)$ and hence they are all isomorphic to $P_{f_{1}}$. For $i \in\left[1, m-f_{1}\right]$, Theorem 13(1) gives $\operatorname{dim} X_{i\left(P_{f_{1}}\right)}=m-i$, which is in $\left[f_{1}, m-1\right]$; hence for every $\ell \in\left[f_{1}, m-1\right]$ there is a unique cell of dimension $\ell$. For $m=n-k$ and $f_{1}=e_{1}-k$ we get (35).

Notice that (32) can be written as $P_{X}(t)=P_{\mathrm{Gr}_{1}\left(e_{2}\right)}(t) P_{\mathrm{Gr}_{1}\left(n-e_{1}\right)}(t)$, where $e_{2}=$ $e_{1}+1$. Surprisingly, this turns out to be a general fact, which we state as the main result of this section:

Theorem 15. Let $X=\operatorname{Gr}_{\left(e_{1}, e_{2}\right)}\left(R_{n}\right)$. The Poincaré polynomial $P_{X}(t)$ of $X$ equals

$$
P_{X}(t)=P_{\mathrm{Gr}_{\left(e_{2}-e_{1}\right)}\left(e_{2}\right)}(t) P_{\mathrm{Gr}_{\left(e_{2}-e_{1}\right)}\left(n-e_{1}\right)}(t) .
$$

Proof. We proceed by induction on $n \geq e_{1} \geq 0$. For $e_{1}=0, X \simeq \operatorname{Gr}_{e_{2}}(n)$ and (37) follows. Let $1 \leq e_{1} \leq e_{2}$. The variety $X$ can be decomposed as

$$
X=Y_{0}^{\prime}(X) \cup X_{1}^{\prime}(X)
$$

where $Y_{0}^{\prime}=Y_{0}^{\prime}(X)$ consists of all preprojective subrepresentations of $R_{n}$ in $X$ and $X_{1}^{\prime}$ consists of subrepresentations of $R_{n}$ in $X$ having a nonzero regular subrepresentation (see (21) and (33)). Moreover, by (24), $X_{1}^{\prime}(X) \simeq \operatorname{Gr}_{\left(e_{1}-1, e_{2}-1\right)}\left(R_{n-1}\right)$ and hence we have

$$
P_{X}(t)=P_{Y_{0}^{\prime}}(t)+P_{\operatorname{Gr}_{\left(e_{1}-1, e_{2}-1\right)}\left(R_{n-1}\right)}(t) .
$$

By the inductive hypothesis (37) holds if and only if

$$
P_{Y_{0}^{\prime}}(t)=\left(P_{\mathrm{Gr}_{\left(e_{2}-e_{1}\right)}\left(e_{2}\right)}(t)-P_{\mathrm{Gr}_{\left(e_{2}-e_{1}\right)}\left(e_{2}-1\right)}(t)\right) P_{\mathrm{Gr}_{\left(e_{2}-e_{1}\right)}\left(n-e_{1}\right)}(t) .
$$

Hence we prove (39). 
We make the following choice: we fix a linear basis $\left\{v_{1}, \ldots, v_{s}\right\}$ of a vector space of dimension $s$ and we let the torus act on $\mathrm{Gr}_{t}(s)$ by $t_{\lambda} v_{i}=\lambda^{i} v_{i}$. We consider the vector subspace of $k^{e_{2}}$ generated by $v_{2}, \ldots, v_{e_{2}}$ and the corresponding embedding $\operatorname{Gr}_{\left(e_{2}-e_{1}\right)}\left(e_{2}-1\right) \subset \operatorname{Gr}_{\left(e_{2}-e_{1}\right)}\left(e_{2}\right)$. With this choice the difference $\operatorname{Gr}_{\left(e_{2}-e_{1}\right)}\left(e_{2}\right) \backslash$ $\operatorname{Gr}_{\left(e_{2}-e_{1}\right)}\left(e_{2}-1\right)$ is $T$-stable and for every point $W$ of it, $\lim _{\lambda \rightarrow 0} t_{\lambda} W$ still belongs to it. The right-hand side of (39) is the Poincaré polynomial (with respect to the cohomology with compact support) of the smooth projective variety

$$
G:=\left(\operatorname{Gr}_{\left(e_{2}-e_{1}\right)}\left(e_{2}\right) \backslash \operatorname{Gr}_{\left(e_{2}-e_{1}\right)}\left(e_{2}-1\right)\right) \times \operatorname{Gr}_{\left(e_{2}-e_{1}\right)}\left(n-e_{1}\right) .
$$

The one-dimensional torus $T$ acts on $G$ and the attracting sets of the $T$-fixed points form a cellular decomposition of $G$. We prove that there exists a bijection between the cells of $Y_{0}^{\prime}$ of dimension $k$ and the cells of $G$ of dimension $k$.

A point of $Y_{0}^{\prime}$ is a direct sum of precisely $\left(e_{2}-e_{1}\right)$ indecomposable preprojective subrepresentations of $R_{n}$ (this follows by considering their dimension vectors). The $T$-fixed points have the form

$$
\left.L:={ }_{k_{1}}\left(P_{r_{1}}\right) \oplus_{k_{2}}\left(P_{r_{2}}\right) \oplus \cdots \oplus{k_{\left(e_{2}-e_{1}\right)}}_{r_{\left(e_{2}-e_{1}\right)}}\right),
$$

where $r_{1}+\cdots+r_{e_{2}-e_{1}}=e_{1}, r_{i} \geq 0$ and ${ }_{k}\left(P_{r}\right)$ has the same meaning as in Section 2.7. In view of Theorem 13 and of (12) the dimension of the attracting cell of $L$ equals

$$
\operatorname{dim} X_{L}=n\left(e_{2}-e_{1}\right)-\sum_{i=1}^{e_{2}-e_{1}} k_{i}-\left(e_{2}-e_{1}\right)^{2}+\sum_{i=1}^{e_{2}-e_{1}} \sum_{j=1}^{i}\left(r_{j}-r_{i}+1\right) .
$$

We consider the set $\alpha(\boldsymbol{e}, k, n)$ which parametrizes the $T$-fixed points of $Y_{0}^{\prime}$ whose attracting set has dimension $k$, i.e.,

$$
\begin{aligned}
\alpha(\boldsymbol{e}, k, n):=\{ & \left(k_{1}, k_{2}, \ldots, k_{e_{2}-e_{1}}, r_{1}, r_{2}, \ldots, r_{e_{2}-e_{1}}\right): \\
& 1 \leq k_{1} \leq k_{1}+r_{1}<k_{2} \leq k_{2}+r_{2}<\cdots<k_{e_{2}-e_{1}} \leq k_{e_{2}-e_{1}}+r_{e_{2}-e_{1}} \leq n, \\
& r_{1}+r_{2}+\cdots+r_{e_{2}-e_{1}}=e_{1}, r_{i} \geq 0, \\
& \left.n\left(e_{2}-e_{1}\right)-\sum_{i=1}^{e_{2}-e_{1}} k_{i}-\left(e_{2}-e_{1}\right)^{2}+\sum_{i=1}^{e_{2}-e_{1}} \sum_{j=1}^{i}\left(r_{j}-r_{i}+1\right)=k\right\} .
\end{aligned}
$$

Now consider the $T$-fixed points of $G$ and their attracting sets. The $T$-fixed points of $\mathrm{Gr}_{t}(s)$ consist of coordinate vector subspaces of dimension $t$ and they are naturally parametrized by tuples $\left(a_{1}, \ldots, a_{t}\right)$ of integers $1 \leq a_{1}<\cdots<a_{t} \leq s$. The corresponding cell $\mathrm{O}_{\left(a_{1}, \ldots, a_{t}\right)}$ has dimension

$$
s-a_{1}-(t-1)+s-a_{2}-(t-2)+\cdots s-a_{t}=t s-\sum_{i=1}^{t} a_{i}-\sum_{i=1}^{t-1} i .
$$

The $T$-fixed points of $\operatorname{Gr}_{\left(e_{2}-e_{1}\right)}\left(e_{2}\right) \backslash \mathrm{Gr}_{\left(e_{2}-e_{1}\right)}\left(e_{2}-1\right)$ are the coordinate vector subspaces of $k^{e_{2}}$ containing $v_{1}$. Hence the following set parametrizes the cells of $G$ 
of dimension $k$ :

$$
\begin{gathered}
\beta(\boldsymbol{e}, k, n):=\left\{\left(a_{1}, a_{2}, \ldots, a_{e_{2}-e_{1}}, b_{2}, b_{3}, \ldots, b_{e_{2}-e_{1}}\right):\right. \\
1 \leq a_{1}<a_{2}<\cdots<a_{e_{2}-e_{1}} \leq n-e_{1}, \quad 2 \leq b_{2}<\cdots<b_{e_{2}-e_{1}} \leq e_{2}, \\
\left.n\left(e_{2}-e_{1}\right)-\sum_{i=1}^{e_{2}-e_{1}} a_{i}-\sum_{i=2}^{e_{2}-e_{1}} b_{i}+\left(e_{2}-e_{1}-1\right)=k\right\} .
\end{gathered}
$$

We consider the map $\beta(\boldsymbol{e}, k, n) \rightarrow \alpha(\boldsymbol{e}, k, n)$ defined by

$$
a_{1} \mapsto k_{1}, \quad a_{i} \mapsto k_{i}-r_{1}-r_{2}-\cdots-r_{i-1}, \quad b_{i} \mapsto \sum_{j=0}^{i-2} r_{\left(e_{2}-e_{1}-j\right)}+i,
$$

for $i \in\left[2, e_{2}-e_{1}\right]$. It is straightforward to verify that this map is a bijection between $\beta(\boldsymbol{e}, k, n)$ and $\alpha(\boldsymbol{e}, k, n)$. It follows that $Y_{0}^{\prime}$ and $G$ have the same Betti numbers and hence (39) follows.

Corollary 16. The Poincaré polynomial of a quiver Grassmannian associated with the indecomposable preprojective $P_{n}$ and the indecomposable preinjective $I_{n}$, where $n \geq 0$, are given by

$$
\begin{aligned}
& P_{\mathrm{Gr} e\left(P_{n}\right)}(t)=P_{\mathrm{Gr}_{e_{1}}\left(e_{2}-1\right)}(t) P_{\mathrm{Gr}_{\left(e_{2}-e_{1}\right)}\left(n+1-e_{1}\right)}(t), \\
& P_{\mathrm{Gr}_{e}\left(I_{n}\right)}(t)=P_{\mathrm{Gr}_{e_{1}}\left(e_{2}+1\right)}(t) P_{\mathrm{Gr}_{\left(e_{2}-e_{1}\right)}\left(n-e_{1}\right)}(t) .
\end{aligned}
$$

Proof. Equality (41) follows from (40) by the isomorphism

$$
\operatorname{Gr}_{\left(e_{1}, e_{2}\right)}\left(I_{n}\right) \simeq \operatorname{Gr}_{\left(n-e_{2}, n+1-e_{1}\right)}\left(P_{n}\right) .
$$

Hence we prove (40). As in the proof of Theorem 15, let $Y_{0}^{\prime}=Y_{0}^{\prime}\left(\operatorname{Gr}_{e}\left(R_{n+1}\right)\right)$ be the subvariety of $\operatorname{Gr}_{e}\left(R_{n+1}\right)$ of all preprojective subrepresentations of $R_{n+1}$ of dimension vector $\boldsymbol{e}$ and let

$$
G^{\prime}:=\operatorname{Gr}_{\left(e_{2}-e_{1}\right)}\left(e_{2}\right) \backslash \operatorname{Gr}_{\left(e_{2}-e_{1}\right)}\left(e_{2}-1\right)
$$

with the convention that $\operatorname{Gr}_{\left(e_{2}-e_{1}\right)}\left(e_{2}-1\right)$ consists of all the elements of $\operatorname{Gr}_{\left(e_{2}-e_{1}\right)}\left(e_{2}\right)$ not containing the first basis vector (as in the proof of Theorem 15). In view of (39) it is sufficient to prove the equalities

$$
\begin{aligned}
& P_{G^{\prime}}(t)=t^{2 e_{1}} P_{\operatorname{Gr}_{\left(e_{2}-e_{1}-1\right)}\left(e_{2}-1\right)}(t), \\
& P_{Y_{0}^{\prime}}(t)=t^{2 e_{1}} P_{\operatorname{Gr}_{e}\left(P_{n}\right)}(t) .
\end{aligned}
$$

The proof of (42) is similar to the proof of Theorem 15: there is an obvious bijection between the cells of $G^{\prime}$ of dimension $k$ and the cells of $\operatorname{Gr}_{\left(e_{2}-e_{1}\right)}\left(e_{2}-1\right)$ of dimension $k-e_{1}$. Let us prove (43). Let $L \in Y_{0}^{\prime}$. Then $L$ is a sum of preprojective subrepresentations of $R_{n+1}$ and $L$ is a subrepresentation of $P_{n} \leq_{Q} R_{n+1}$. By looking at the quotients $R_{n+1} / L$ and $P_{n} / L$ and using Lemma 5 one gets

$$
\operatorname{dim} \operatorname{Hom}\left(L, R_{n+1} / L\right)^{+}=\operatorname{dim} \operatorname{Hom}\left(L, P_{n} / L\right)^{+}+e_{1} .
$$


Hence the cells of $Y_{0}^{\prime}$ of dimension $k$ are in bijection with the cells of $\operatorname{Gr}_{e}\left(P_{n}\right)$ of dimension $k-e_{1}$ and (43) holds.

Since $\chi(X)=P_{X}(1)$ for a projective variety $X$ and $\chi\left(\mathrm{Gr}_{t}(s)\right)=\left(\begin{array}{l}s \\ t\end{array}\right)$, we recover the following result, of which alternative proofs have also appeared in [Cerulli Irelli 2011b; Zelevinsky 2007; Szanto 2011; Poettering 2010].

Corollary 17 [Caldero and Zelevinsky 2006].

$$
\begin{aligned}
\chi\left(\operatorname{Gr}_{e}\left(R_{n}\right)\right) & =\left(\begin{array}{c}
e_{2} \\
e_{1}
\end{array}\right)\left(\begin{array}{c}
n-e_{1} \\
e_{2}-e_{1}
\end{array}\right), \\
\chi\left(\operatorname{Gr}_{e}\left(P_{n}\right)\right) & =\left(\begin{array}{c}
e_{2}-1 \\
e_{1}
\end{array}\right)\left(\begin{array}{c}
n+1-e_{1} \\
e_{2}-e_{1}
\end{array}\right), \\
\chi\left(\operatorname{Gr}_{e}\left(I_{n}\right)\right) & =\left(\begin{array}{c}
e_{2}+1 \\
e_{1}
\end{array}\right)\left(\begin{array}{c}
n-e_{1} \\
e_{2}-e_{1}
\end{array}\right) .
\end{aligned}
$$

\section{Applications to cluster algebras}

To a finite quiver $Q$ without loops and 2-cycles is associated a (coefficient-free) cluster algebra $\mathscr{A}_{Q}$ ([Fomin and Zelevinsky 2002]; see also [Fomin and Zelevinsky 2003b] and [Keller 2010] for excellent surveys). This is a $\mathbb{Z}$-subalgebra of the field $\mathscr{F}_{\mathrm{F}}$ of rational functions in $n$ variables ( $n$ being the number of vertices of $Q$ ), generated by its cluster variables. The cluster variables are grouped into free-generating sets of $\mathscr{F}$ called clusters. The cluster monomials are monomials in cluster variables belonging to the same cluster. An atomic basis of $\mathscr{A}_{Q}$ is a $\mathbb{Z}$-basis $\boldsymbol{B}$ of it such that the positive linear combinations of elements of $\boldsymbol{B}$ coincide with the semiring of positive elements of $\mathscr{A}_{Q}$ (elements that are positive Laurent polynomials in every cluster of $\mathscr{A}_{Q}$ ); see [Sherman and Zelevinsky 2004]. The existence of such a basis has been proved only in a few cases:

- If $Q$ is of type $A D E$, cluster monomials form an atomic basis of $\mathscr{A}_{Q}$ [Cerulli Irelli 2011a; Cerulli Irelli and Labardini-Fragoso 2011].

- When $Q$ is the Kronecker quiver, atomic bases exist coefficients and consist of cluster monomials together with extra elements $\left\{z_{n}: n \geq 1\right\}$ [Sherman and Zelevinsky 2004].

- when $Q$ is of type $A_{2}^{(1)}$, the atomic bases of $\mathscr{A}_{Q}$ have been found in [Cerulli Irelli 2009] (for every choice of the coefficients) and consist of cluster monomials together with some extra elements $\left\{u_{n}: n \geq 1\right\}$ possibly multiplied by particular cluster variables.

We notice that under the Caldero-Keller bijection, cluster monomials correspond, via the Caldero-Chapoton map, to rigid $Q$-representations and the quiver Grassmannians associated with rigid representations are smooth. In the next two sections 
we propose a "truncation" of the Caldero-Chapoton map that gives a geometric realization of the extra elements $\left\{z_{n}\right\},\left\{u_{n}\right\}$.

3.1. Type $\boldsymbol{A}_{1}^{(\mathbf{1})}$. Let $\mathscr{F}=\boldsymbol{Q}\left(x_{1}, x_{2}\right)$ be the field of rational functions in two independent variables $x_{1}$ and $x_{2}$ with rational coefficients. We recursively define elements $\left\{x_{k}: k \in \mathbb{Z}\right\}$ of $\mathscr{F}$ by

$$
x_{k} x_{k+2}=x_{k+1}^{2}+1, \quad k \in \mathbb{Z} .
$$

Let $\mathscr{A}$ be the $\mathbb{Z}$-subalgebra of $\mathscr{F}$ generated by all the $x_{k}$ 's $(k \in \mathbb{Z})$. By [Sherman and Zelevinsky 2004] the algebra $\mathscr{A}$ is the coefficient-free cluster algebra associated with the Kronecker quiver. The pairs $\left\{x_{k}, x_{k+1}\right\}, k \in \mathbb{Z}$, are free generating sets of $\mathscr{F}$ and form the clusters of $\mathscr{A}$. Monomials $x_{k}^{a} x_{k+1}^{b}, a, b \geq 0, k \in \mathbb{Z}$, are called the cluster monomials of $\mathscr{A}$.

Caldero and Zelevinsky [2006] defined the rational function

$$
s_{n}:=C C\left(R_{n}\right)
$$

for every $n \geq 1$, where $R_{n}$ is a regular indecomposable $Q$-representation of dimension $(n, n)$. They have proved that the set $\mathscr{S}:=$ cluster monomials $\} \cup\left\{s_{n}: n \geq 1\right\}$ is a $\mathbb{Z}$-basis of $\mathscr{A}$.

The atomic basis $\boldsymbol{B}$ defined in the introduction and the basis $\mathscr{S}$ are related by (see [Caldero and Zelevinsky 2006])

$$
z_{n}=s_{n}-s_{n-2}
$$

for $n \geq 1$ and the convention that $s_{-k}=0$ for $k>0, s_{0}:=1$.

Theorem 18. The element $z_{n}$ has the Laurent expansion

$$
z_{n}=\frac{\sum_{\boldsymbol{e}} \chi\left(\mathrm{Gr}_{\boldsymbol{e}}\left(R_{n}\right)^{S m}\right) x_{1}^{2\left(n-e_{2}\right)} x_{2}^{2 e_{1}}}{x_{1}^{n} x_{2}^{n}}
$$

where $\mathrm{Gr}_{\boldsymbol{e}}\left(R_{n}\right)^{\text {Sm }}$ denotes the smooth part of $\mathrm{Gr}_{\boldsymbol{e}}\left(R_{n}\right)$.

Proof. In view of Theorem $8, \operatorname{Gr}_{e}\left(R_{n}\right)^{S m}=X_{0} \backslash X_{1}$ where $X_{0}=\operatorname{Gr}_{e}\left(R_{n}\right)$ and $X_{1}=\operatorname{Gr}_{\left(e_{1}-1, e_{2}-1\right)}\left(R_{n-2}\right)$ and hence

$$
\chi\left(\operatorname{Gr}_{\boldsymbol{e}}\left(R_{n}\right)^{S m}\right)=\chi\left(\mathrm{Gr}_{\boldsymbol{e}}\left(R_{n}\right)\right)-\chi\left(\operatorname{Gr}_{\left(e_{1}-1, e_{2}-1\right)}\left(R_{n-2}\right)\right) .
$$

It is now easy to check that the right-hand side of (48) satisfies (47).

3.2. Type $\boldsymbol{A}_{2}^{(\mathbf{1})}$. We now briefly recall the construction of the atomic basis of cluster algebras of type $A_{2}^{(1)}$ from [Cerulli Irelli 2009]. Let $\mathscr{F}=\mathbb{Q}\left(x_{1}, x_{2}, x_{3}\right)$ be the field of rational functions in three (commuting) independent variables $x_{1}, x_{2}$ and $x_{3}$ with rational coefficients. Recursively define elements $x_{m} \in \mathscr{F}$ for $m \in \mathbb{Z}$ by the relation

$$
x_{m} x_{m+3}=x_{m+1} x_{m+2}+1 .
$$


Define also the elements $w, z \in \mathscr{F}$ by

$$
w=\frac{x_{1}+x_{3}}{x_{2}}, \quad z=\frac{x_{1} x_{2}+x_{2} x_{3}+1}{x_{1} x_{3}} .
$$

The (coefficient-free) cluster algebra $\mathscr{A}$ of type $A_{2}^{(1)}$ is the $\mathbb{Z}$-subalgebra of $\mathscr{F}$ generated by all the $x_{m}$ 's, $w$ and $z$ (see also [Fomin and Zelevinsky 2002, Example 7.8]). This is the cluster algebra $\mathscr{A}=\mathscr{A}_{Q_{2}}$ associated with the affine quiver

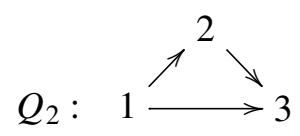

of type $A_{2}^{(1)}$. The elements $x_{m}, m \in \mathbb{Z}, w$ and $z$ are the cluster variables of $\mathscr{A}$. The sets $\left\{x_{m}, x_{m+1}, x_{m+2}\right\},\left\{x_{2 m}, z, x_{2 m+2}\right\}$ and $\left\{x_{2 m-1}, w, x_{2 m+1}\right\}, m \in \mathbb{Z}$, are the clusters of $\mathscr{A}$. The cluster monomials are monomials in cluster variables belonging to the same cluster. The exchange graph of $\mathscr{A}$ is the brick wall shown here:

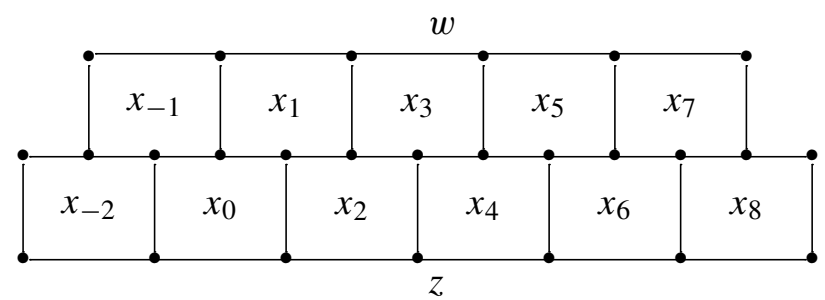

It has clusters as vertices and an edge between two vertices if the corresponding clusters share precisely two cluster variables. In this figure the cluster variables of a cluster $\mathscr{C}$ label the regions surrounding the vertex corresponding to $\mathscr{C}$.

Define elements $u_{n}, n \geq 0$, of $\mathscr{F}$ by the recursion

$$
u_{0}=2, \quad u_{1}=z w-2, \quad u_{n+1}=u_{1} u_{n}-u_{n-1} \quad \text { for } n \geq 1 .
$$

In [Cerulli Irelli 2009] it is shown that the set

$$
\boldsymbol{B}=\{\text { cluster monomials }\} \cup\left\{u_{n} w^{k}, u_{n} z^{k}: n \geq 1, k \geq 0\right\}
$$

is an atomic basis of $\mathscr{A}$. We now realize the elements $u_{n}$ as images of the CalderoChapoton map. Recall that for a representation $M$ of the quiver $Q_{2}$ of (51) the Caldero-Chapoton map $C C(M)$ is the following (see [Caldero and Zelevinsky 2006]):

$$
C C(M)=\frac{\sum_{e} \chi\left(\mathrm{Gr}_{\boldsymbol{e}}(M)\right) x_{1}^{d_{2}+d_{3}-e_{2}-e_{3}} x_{2}^{d_{3}-e_{3}+e_{1}} x_{3}^{e_{1}+e_{2}}}{x_{1}^{d_{1}} x_{2}^{d_{2}} x_{3}^{d_{3}}}
$$


where $\left(d_{1}, d_{2}, d_{3}\right)$ is the dimension vector of $M$. For every $n \geq 1$ let $R_{n, 2}$ be the indecomposable regular $Q_{2}$-representation in an homogeneous tube, i.e.,

$$
R_{n, 2}=\quad k^{n} \stackrel{\nearrow_{J_{n}(0)}}{\longrightarrow} k^{n}
$$

where $J_{n}(0)$ denotes the $n \times n$ indecomposable nilpotent Jordan block.

Theorem 19. For every $n \geq 1$,

$$
u_{n}=\frac{\sum_{\boldsymbol{e}} \chi\left(\mathrm{Gr}_{\boldsymbol{e}}\left(R_{n, 2}\right)^{S m}\right) x_{1}^{2 n-e_{2}-e_{3}} x_{2}^{n-e_{3}+e_{1}} x_{3}^{e_{1}+e_{2}}}{x_{1}^{n} x_{2}^{n} x_{3}^{n}}
$$

where $\mathrm{Gr}_{\boldsymbol{e}}\left(R_{n, 2}\right)^{\text {Sm }}$ denotes the smooth part of the quiver Grassmannian $\mathrm{Gr}_{\boldsymbol{e}}\left(R_{n, 2}\right)$, $\boldsymbol{e}:=\left(e_{1}, e_{2}, e_{3}\right)$.

Proof. Let $u_{n}^{\prime}$ denote the right-hand side of (54). We prove that $\left\{u_{n}^{\prime}\right\}$ satisfies (52). We consider the fibration of projective varieties

$$
f: \operatorname{Gr}_{\left(e_{1}, e_{2}, e_{3}\right)}\left(R_{n, 2}\right) \rightarrow \operatorname{Gr}_{\left(e_{1}, e_{3}\right)}\left(R_{n}\right)
$$

taking $\left(N_{1}, N_{2}, N_{3}\right)$ to $\left(N_{1}, N_{3}\right)$; its fiber is $\operatorname{Gr}_{\left(e_{2}-e_{1}\right)}\left(e_{3}-e_{1}\right)$. In particular

$$
\operatorname{Gr}_{\left(e_{1}, e_{2}, e_{3}\right)}\left(R_{n, 2}\right)^{S m}=f^{-1}\left(\operatorname{Gr}_{\left(e_{1}, e_{3}\right)}\left(R_{n}\right)^{S m}\right) \text {. }
$$

Using this it is straightforward to check that

$$
u_{n}^{\prime}=z_{n}\left(x_{1} w^{-1 / 2}, x_{3} w^{-1 / 2}\right)
$$

where the right-hand side means that (48) should be computed with the substitutions $x_{1} \mapsto x_{1} w^{-1 / 2}$ and $x_{2} \mapsto x_{3} w^{-1 / 2}$, and $w^{-1 / 2}$ is a formal variable whose square is $w^{-1}$. The defining equations (4) yield

$$
u_{1}^{\prime}=z_{1}\left(x_{1} w^{-1 / 2}, x_{3} w^{-1 / 2}\right)=\frac{x_{1}^{2}+x_{3}^{2}+w}{x_{1} x_{2}}=z w-2
$$

and $u_{n+1}^{\prime}=u_{1}^{\prime} u_{n}^{\prime}-u_{n-1}^{\prime}$, so $u_{n}^{\prime}=u_{n}$ for every $n \geq 1$.

\section{Conclusions}

The study of quiver Grassmannians from a geometric point of view has provided interesting information about cluster algebras. After this study it is natural to define the following slight modification of the Caldero-Chapoton map: for $k \geq 0$,

$$
C C^{(k)}(M):=\frac{\sum_{\boldsymbol{e}} \chi\left(\mathrm{Gr}_{\boldsymbol{e}}(M)^{(k)}\right) x_{1}^{2\left(d_{2}-e_{2}\right)} x_{2}^{2 e_{1}}}{x_{1}^{d_{1}} x_{2}^{d_{2}}},
$$


where

$$
\operatorname{Gr}_{\boldsymbol{e}}(M)^{(k)}:=\left\{N \in \operatorname{Gr}_{\boldsymbol{e}}(M): \operatorname{dim} \operatorname{Ext}^{1}(N, M / N)=k\right\} .
$$

In the case of the Kronecker quiver the map $C C^{(0)}(M)$ is defined by the smooth part of the quiver Grassmannians associated with $M$. This is not a general fact and it is studied in [Cerulli Irelli et al. 2012]. In that paper we study the relationship between the maps $C C^{(k)}$ and the transverse quiver Grassmannian introduced in [Dupont 2010].

We notice that for a rigid representation $M$ we have $C C^{(0)}(M)=C C(M)$. Moreover it is easy to check that if $\operatorname{Ext}^{1}(M, N)=\operatorname{Ext}^{1}(N, M)=0$ then $C C^{0}(M \oplus N)=$ $C C^{0}(M) C C^{0}(N)$. In particular the elements $u_{n} w$ and $u_{n} z$ of the basis (53) are $C C^{0}\left(R_{n, 2} \oplus W\right)$ and $C C^{0}\left(R_{n, 2} \oplus Z\right)$ where $W$ and $Z$ are the indecomposable regular rigid $Q_{2}$-representations. We trust that this approach can help in the determination of atomic bases of cluster algebras of affine type of higher rank.

\section{Acknowledgements}

We thank Andrea Maffei for useful conversations.

Cerulli Irelli thanks the organizers of the "Advanced School and Conference on Homological and Geometrical Methods in Representation Theory" that took place in Trieste (Italy) at the beginning of 2010 for the opportunity to present this project in front of several experts and to spend a fruitful time there.

We are grateful to Andrei Zelevinsky for having pointed out the formulas in [Szanto 2011], and to Grégoire Dupont for his comments about transverse quiver Grassmannians.

\section{References}

[Assem et al. 2006] I. Assem, D. Simson, and A. Skowroński, Elements of the representation theory of associative algebras, vol. 1: Techniques of representation theory, London Math. Soc. Student Texts 65, Cambridge University Press, Cambridge, 2006. MR 2006j:16020 Zbl 1092.16001

[Berenstein et al. 2005] A. Berenstein, S. Fomin, and A. Zelevinsky, "Cluster algebras, III: Upper bounds and double Bruhat cells”, Duke Math. J. 126:1 (2005), 1-52. MR 2005i:16065

[Białynicki-Birula 1973] A. Białynicki-Birula, "Some theorems on actions of algebraic groups", Ann. of Math. (2) 98 (1973), 480-497. MR 51 \#3186 Zbl 0275.14007

[Caldero and Chapoton 2006] P. Caldero and F. Chapoton, "Cluster algebras as Hall algebras of quiver representations”, Comment. Math. Helv. 81:3 (2006), 595-616. MR 2008b:16015 Zbl 1119. 16013

[Caldero and Keller 2006] P. Caldero and B. Keller, "From triangulated categories to cluster algebras, II”, Ann. Sci. École Norm. Sup. (4) 39:6 (2006), 983-1009. MR 2008m:16031 Zbl 1115.18301

[Caldero and Keller 2008] P. Caldero and B. Keller, "From triangulated categories to cluster algebras", Invent. Math. 172:1 (2008), 169-211. MR 2009f:16027 Zbl 1141.18012

[Caldero and Reineke 2008] P. Caldero and M. Reineke, "On the quiver Grassmannian in the acyclic case”, J. Pure Appl. Algebra 212:11 (2008), 2369-2380. MR 2009f:14102 Zbl 1153.14032 
[Caldero and Zelevinsky 2006] P. Caldero and A. Zelevinsky, "Laurent expansions in cluster algebras via quiver representations", Mosc. Math. J. 6:3 (2006), 411-429. MR 2008j:16045 Zbl 1133.16012

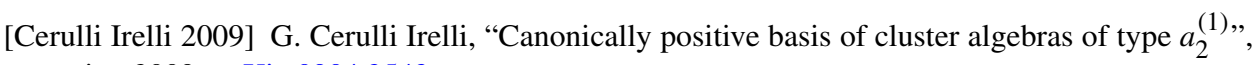
preprint, 2009. arXiv 0904.2543

[Cerulli Irelli 2011a] G. Cerulli Irelli, "Positivity in skew-symmetric cluster algebras of finite type", preprint, 2011. arXiv 1102.3050

[Cerulli Irelli 2011b] G. Cerulli Irelli, "Quiver grassmannians associated with string modules", $J$. Algebraic Combin. 33:2 (2011), 259-276. MR 2765325 Zbl 05852822

[Cerulli Irelli and Labardini-Fragoso 2011] G. Cerulli Irelli and D. Labardini-Fragoso, "Quivers with potentials associated to triangulated surfaces, III: tagged triangulations and cluster monomials", (2011). arXiv 1108.1774

[Cerulli Irelli et al. 2012] G. Cerulli Irelli, G. Dupont, and F. Esposito, "A homological interpretation of the transverse quiver grassmannians", Algebr. Represent. Theory (2012).

[Chriss and Ginzburg 1997] N. Chriss and V. Ginzburg, Representation theory and complex geometry, Birkhäuser, Boston, 1997. MR 98i:22021 Zbl 0879.22001

[Crawley-Boevey 1989] W. W. Crawley-Boevey, "Maps between representations of zero-relation algebras", J. Algebra 126:2 (1989), 259-263. MR 90k:16035 Zbl 0685.16018

[Derksen et al. 2010] H. Derksen, J. Weyman, and A. Zelevinsky, "Quivers with potentials and their representations, II: Applications to cluster algebras”, J. Amer. Math. Soc. 23:3 (2010), 749-790. MR 2629987

[Dupont 2010] G. Dupont, "Transverse quiver grassmannians and bases in affine cluster algebras", Algebra Number Theory 4:5 (2010), 599-624. MR 2011i:13027 Zbl 05779836

[Fomin and Zelevinsky 2002] S. Fomin and A. Zelevinsky, "Cluster algebras, I: Foundations", J. Amer. Math. Soc. 15:2 (2002), 497-529. MR 2003f:16050

[Fomin and Zelevinsky 2003a] S. Fomin and A. Zelevinsky, "Cluster algebras. II. Finite type classification”, Invent. Math. 154:1 (2003), 63-121. MR 2004m:17011

[Fomin and Zelevinsky 2003b] S. Fomin and A. Zelevinsky, "Cluster algebras: notes for the CDM-03 conference", pp. 1-34 in Current developments in mathematics, 2003, edited by B. Mazur et al., Int. Press, Somerville, MA, 2003. MR 2005m:05235 Zbl 1119.05108

[Fomin and Zelevinsky 2007] S. Fomin and A. Zelevinsky, "Cluster algebras, IV: Coefficients", Compos. Math. 143:1 (2007), 112-164. MR 2008d:16049

[Geiß et al. 2012] C. Geiß, B. Leclerc, and J. Schröer, "Generic bases for cluster algebras and the chamber ansatz", J. Amer. Math. Soc. 25:1 (2012), 21-76. MR 2833478

[Hernandez and Leclerc 2010] D. Hernandez and B. Leclerc, "Cluster algebras and quantum affine algebras”, Duke Math. J. 154:2 (2010), 265-341. MR 2011g:17027 Zbl 05788166

[Keller 2010] B. Keller, "Cluster algebras, quiver representations and triangulated categories", pp. 76-160 in Triangulated categories, edited by T. Holm et al., London Math. Soc. Lecture Note Ser. 375, Cambridge Univ. Press, Cambridge, 2010. MR 2011h:13033 Zbl 1215.16012

[Kronecker 1890] L. Kronecker, "Algebraische Reduction der Schaaren bilinearer Formen", Sitzungsber. Akad. Berlin (1890), 1225-1237. Reprinted as pp. 139-156 in his Werke, vol. 3, Teubner, Leipzig, 1931. JFM 22.0169.01

[Lampe 2011] P. Lampe, "A quantum cluster algebra of Kronecker type and the dual canonical basis", Int. Math. Res. Not. 2011:13 (2011), 2970-3005. MR 2817684 Zbl 05931742

[Nakajima 2011] H. Nakajima, "Quiver varieties and cluster algebras”, Kyoto J. Math. 51:1 (2011), 71-126. MR 2784748 Zbl 1223.13013 
[Poettering 2010] N. Poettering, "Euler characteristic of quiver Grassmannians and Ringel-Hall algebras of string algebras", preprint, 2010. To appear in Algebr. Represent. Theory. arXiv 1002.3040

[Ringel 1998] C. M. Ringel, "Exceptional modules are tree modules", Linear Algebra Appl. 275/276 (1998), 471-493. MR 2000c:16020 Zbl 0964.16014

[Sherman and Zelevinsky 2004] P. Sherman and A. Zelevinsky, "Positivity and canonical bases in rank 2 cluster algebras of finite and affine types", Mosc. Math. J. 4:4 (2004), 947-974. MR 2006c: 16052 Zbl 1103.16018

[Szanto 2011] C. Szanto, "On the cardinalities of Kronecker quiver grassmannians", Math. Z. 269:3-4 (2011), 833-846.

[Zelevinsky 2007] A. Zelevinsky, "Semicanonical basis generators of the cluster algebra of type

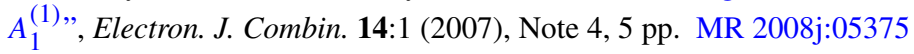

Communicated by Andrei Zelevinsky

Received 2010-03-22 Revised 2010-09-14 Accepted 2010-10-30

cerulli@mat.uniroma1.it

Current address:

esposito@math.unipd.it
Università degli studi di Padova, Dipartimento di Matematica Pura ed Applicata, Via Trieste 63, I-35121 Padova, Italy

Università di Roma "La Sapienza", Dipartimento di Matematica, Piazzale Aldo Moro 5, I-00185 Roma, Italy

Università degli studi di Padova, Dipartimento di Matematica Pura ed Applicata, Via Trieste 63, I-35121 Padova, Italy 


\title{
Sur le groupe de Chow de codimension deux des variétés sur les corps finis
}

\author{
Alena Pirutka
}

En utilisant la construction de Colliot-Thélène et Ojanguren, on donne un exemple d'une variété projective et lisse géométriquement rationnelle $X$, définie sur un corps fini $\mathbb{F}_{p}$, telle que d'une part le groupe $H_{\mathrm{nr}}^{3}(X, \mathbb{Z} / 2)$ est non nul et, d'autre part, l'application $C H^{2}(X) \rightarrow C H^{2}\left(X \times_{\mathbb{F}_{p}} \overline{\mathbb{F}}_{p}\right)^{\operatorname{Gal}\left(\overline{\mathbb{F}}_{p} / \mathbb{F}_{p}\right)}$ n'est pas surjective.

Using a construction of Colliot-Thélène and Ojanguren, we exhibit an example of a smooth projective geometrically rational variety $X$ defined over a finite field $\mathbb{F}_{p}$, such that the group $H_{\mathrm{nr}}^{3}(X, \mathbb{Z} / 2)$ is nonzero and the map $C H^{2}(X) \rightarrow$ $C H^{2}\left(X \times_{\mathbb{F}_{p}} \overline{\mathbb{F}}_{p}\right)^{\mathrm{Gal}\left(\overline{\mathbb{F}}_{p} / \mathbb{F}_{p}\right)}$ is not surjective.

Soit $\mathbb{F}_{p}$ un corps fini de cardinal $p$. Soit $\overline{\mathbb{F}}_{p}$ une clôture algébrique de $\mathbb{F}_{p}$ et soit $G=\operatorname{Gal}\left(\overline{\mathbb{F}}_{p} / \mathbb{F}_{p}\right)$ le groupe de Galois absolu. Soit $X$ une $\mathbb{F}_{p}$-variété projective et lisse, géométriquement connexe, de dimension $d$ et soit $\bar{X}=X \times_{\mathbb{F}_{p}} \overline{\mathbb{F}}_{p}$. On dispose d'une application naturelle

$$
C H^{i}(X) \rightarrow C H^{i}(\bar{X})^{G}
$$

entre les groupes de Chow des cycles de codimension $i$ sur $X$ (resp. sur $\bar{X}$ ) modulo l'équivalence rationnelle. Cette application est surjective pour $i=0,1, d$ (voir remarque 3.1). On peut se demander s'il en est ainsi pour $2 \leq i<d$.

Dans cet article, on donne un contre-exemple pour $i=2$. Dans ce cas, des arguments de $K$-théorie algébrique [Kahn 1996] permettent de faire un lien entre le conoyau de l'application $\mathrm{CH}^{2}(X) \rightarrow C H^{2}(\bar{X})^{G}$ et le groupe de cohomologie non ramifiée $H_{\mathrm{nr}}^{3}\left(X, \mathbb{Q}_{l} / \mathbb{Z}_{l}(2)\right)$. On montre dans la $2^{\text {ème }}$ partie qu'il suffit d'assurer que ce dernier groupe est non nul. Pour ce faire, les techniques développées par Colliot-Thélène et Ojanguren [1989] sont disponibles. En utilisant leur méthode, on construit ainsi dans la $3^{3 e ̀ m e}$ partie une variété projective lisse $X$ géométriquement connexe définie sur un corps fini $\mathbb{F}_{p}$ convenable, telle que

$$
\text { l'application } \mathrm{CH}^{2}(X) \rightarrow C H^{2}(\bar{X})^{G} n^{\prime} \text { 'est pas surjective. }
$$

MSC2000: 14C25.

Mots-clefs: groupes de Chow, cohomologie non ramifiée, Chow groups, unramified cohomology. 
Plus précisément, $X$ est une variété géométriquement rationnelle de dimension 5 , admettant un morphisme vers $\mathbb{P}_{\mathbb{F}_{p}}^{2}$ à fibre générique une quadrique lisse «voisine» de Pfister. Notre méthode permet d'obtenir de tels exemples sur des corps finis $\mathbb{F}_{p}$ pour une infinité de nombres premiers $p$.

\section{Notations et rappels}

Notations. Étant donné un corps $k$, on note $k^{*}$ le groupe multiplicatif $k-\{0\}, \bar{k}$ une clôture séparable de $k$ et $G=\operatorname{Gal}(\bar{k} / k)$ le groupe de Galois absolu. On note $\mathbb{F}_{p}$ le corps fini de cardinal $p$.

Si $X$ est une variété algébrique définie sur un corps $k$, on note $\bar{X}=X_{\bar{k}}=X \times{ }_{k} \bar{k}$. Si $X$ est intègre, on note $k(X)$ son corps des fractions et si $X$ est géométriquement intègre, on note $\bar{k}(X)$ le corps des fractions de $\bar{X}$. On dit que $X$ est $k$-rationnelle si $X$ est birationnelle à $\mathbb{P}_{k}^{n}$ et on dit que $X$ est géométriquement rationnelle si $\bar{X}$ est $\bar{k}$-rationnelle.

Pour une $k$-variété intègre $X$ et $i$ un entier, on note $X^{(i)}$ l'ensemble des points de $X$ de codimension $i$ et on note $C H^{i}(X)$ le groupe des cycles de codimension $i$ modulo l'équivalence rationnelle.

Si $A$ est un groupe abélien et $n$ est un entier, on note $A[n]$ le sous-groupe de $A$ formé par les éléments annulés par $n$. Pour $l$ un nombre premier, on note $A\{l\}$ le sous-groupe de torsion $l$-primaire.

Pour $M$ un $G$-module continu discret on note $H^{i}(k, M)=H^{i}(G, M)$ le $i$-ème groupe de cohomologie galoisienne et on note $M^{G}=H^{0}(k, M)$ le sous-groupe formé par les éléments invariants par $G$.

Rappels de cohomologie étale. Étant donnés un corps $k$ et un entier $n$ inversible sur $k$, on note $\mu_{n}$ le $k$-schéma en groupes (étale) des racines $n$-ièmes de l'unité. Pour $j$ un entier positif, on note $\mu_{n}^{\otimes j}=\mu_{n} \otimes \cdots \otimes \mu_{n}$ ( $j$ fois). On pose $\mu_{n}^{\otimes j}=$ $\operatorname{Hom}_{k-g r}\left(\mu_{n}^{\otimes(-j)}, \mathbb{Z} / n\right)$ si $j$ est négatif et $\mu_{n}^{\otimes 0}=\mathbb{Z} / n$. Ces $k$-schémas en groupes donnent des faisceaux étales, notés encore $\mu_{n}^{\otimes j}$, sur toute $k$-variété $X$. On note $H^{i}\left(X, \mu_{n}^{\otimes j}\right)$ les groupes de cohomologie étale de $X$ à valeurs dans $\mu_{n}^{\otimes j}$. Lorsque $n=2$, on a un isomorphisme canonique $\mu_{2}^{\otimes j} \stackrel{\sim}{\rightarrow} \mathbb{Z} / 2$ pour tout $j$.

Définition 1.1. Pour $X$ une $k$-variété intègre, un entier naturel $j \geq 1$ et $i \in \mathbb{Z}$ un entier relatif, on définit les groupes de cohomologie non ramifiée

$H_{\mathrm{nr}}^{j}\left(X, \mu_{n}^{\otimes i}\right) \stackrel{\text { déf }}{=} H_{\mathrm{nr}}^{j}\left(k(X) / k, \mu_{n}^{\otimes i}\right)=\bigcap_{A} \operatorname{Ker}\left[H^{j}\left(k(X), \mu_{n}^{\otimes i}\right) \stackrel{\partial_{j, A}}{\rightarrow} H^{j-1}\left(k_{A}, \mu_{n}^{\otimes i-1}\right)\right]$.

Dans cette formule, $A$ parcourt les anneaux de valuation discrète de rang un, de corps des fractions $k(X)$, contenant le corps $k$. Le corps résiduel d'un tel anneau $A$ est noté $k_{A}$ et l'application $\partial_{j, A}$ est l'application résidu. 
Lorsque $X$ est propre et lisse, les résultats de Bloch et Ogus permettent d'identifier le groupe $H_{\mathrm{nr}}^{j}\left(X, \mu_{n}^{\otimes i}\right)$ au groupe de cohomologie de Zariski $H^{0}\left(X, \mathscr{H}^{j}\left(\mu_{n}^{\otimes i}\right)\right)$, où $\mathscr{H}^{j}\left(\mu_{n}^{\otimes i}\right)$ désigne le faisceau de Zariski sur $X$ associé au préfaisceau $U \mapsto$ $H^{j}\left(U, \mu_{n}^{\otimes i}\right)$ [Colliot-Thélène 1995].

On note $H^{i}(X, \mathbb{Q} / \mathbb{Z}(j))$ et $H^{i}\left(X, \mathbb{Q}_{l} / \mathbb{Z}_{l}(j)\right)$ la limite inductive des groupes $H^{i}\left(X, \mu_{n}^{\otimes j}\right)$ lorsque $n$ varie respectivement parmi les entiers et parmi les puissances d'un nombre premier $l \neq$ car. $k$.

On définit de même les groupes $H_{\mathrm{nr}}^{i}(X, \mathbb{Q} / \mathbb{Z}(j))$ et $H_{\mathrm{nr}}^{i}\left(X, \mathbb{Q}_{l} / \mathbb{Z}_{l}(j)\right)$, comme limites inductives des groupes $H_{\mathrm{nr}}^{i}\left(X, \mu_{n}^{\otimes j}\right)$.

On note $\mathbb{G}_{m}$ le groupe multiplicatif sur un schéma $X$ et le faisceau étale ainsi défini. On écrit $\operatorname{Br} X=H_{\text {ett }}^{2}\left(X, \mathbb{G}_{m}\right)$ pour le groupe de Brauer cohomologique de $X$ et $\operatorname{Pic}(X)=H_{\text {Zar }}^{1}\left(X, O_{X}^{*}\right) \simeq H_{\text {ét }}^{1}\left(X, \mathbb{G}_{m}\right)$ pour le groupe de Picard.

Rappels de $K$-théorie. Pour $X$ un schéma noethérien et $j$ un entier positif on note $\mathscr{K}_{j}$ le faisceau de Zariski associé au préfaisceau $U \mapsto K_{j}\left(H^{0}\left(U, O_{U}\right)\right)$, le groupe $K_{j}(A)$ étant celui associé par Quillen [1973] à l'anneau $A$.

Lorsque $X$ est une variété lisse sur un corps $k$, la conjecture de Gersten, établie par Quillen, permet de calculer les groupes de cohomologie de Zariski $H^{i}\left(X, \mathscr{K}_{j}\right)$ comme les groupes de cohomologie du complexe de Gersten. Lorsque $j=2$, qui est le cas qui nous intéresse dans la suite, ce complexe s'écrit

$$
K_{2} k(X) \stackrel{d_{2}}{\rightarrow} \bigoplus_{x \in X^{(1)}} k(x)^{*} \stackrel{d_{1}}{\rightarrow} \bigoplus_{x \in X^{(2)}} \mathbb{Z},
$$

où l'application $d_{2}$ est donnée par le symbole modéré et l'application $d_{1}$ est obtenue par la somme des flèches diviseurs après normalisation des variétés considérées. On a ainsi $H^{0}\left(X, \mathscr{K}_{2}\right)=\operatorname{Ker} d_{2}$ et $H^{1}\left(X, \mathscr{K}_{2}\right)=\operatorname{Ker} d_{1} / \operatorname{Im} d_{2}$.

Étant donné un corps $k$, le groupe $K_{2} k$ coïncide avec le groupe de $K$-théorie de Milnor $K_{2}^{M} k$, quotient de $k^{*} \otimes_{\mathbb{Z}} k^{*}$ par le sous-groupe engendré par les éléments $a \otimes b$ avec $a+b=1$.

Cette description permet de voir que pour $X$ une variété lisse sur un corps $k$ on a une flèche naturelle

$$
\operatorname{Pic}(X) \otimes k^{*} \rightarrow H^{1}\left(X, \mathscr{K}_{2}\right) .
$$

En effet, on a le diagramme commutatif suivant :

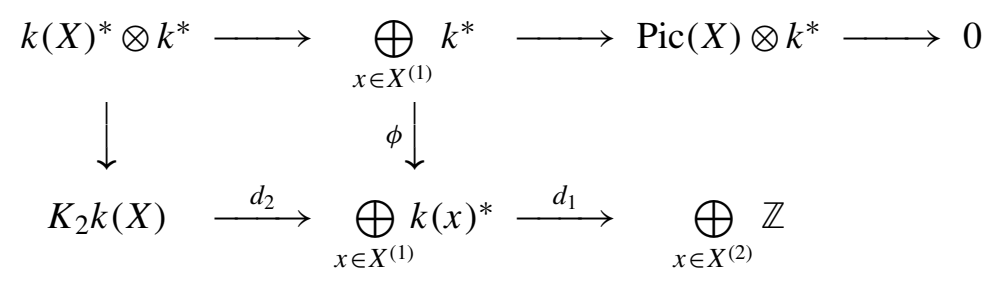


où la première ligne est obtenue à partir de la suite exacte définissant le groupe $\operatorname{Pic}(X)$ par tensorisation avec $k^{*}$. On vérifie que la composé $d_{1} \circ \phi$ vaut zéro, ce qui permet de définir la flèche $\operatorname{Pic}(X) \otimes k^{*} \rightarrow H^{1}\left(X, \mathscr{K}_{2}\right)$ par chasse au diagramme.

\section{Comparaison entre groupes de Chow en codimension deux et cohomologie non ramifiée en degré trois}

Dans cette partie on donne la preuve du théorème suivant :

Théorème 2.1. Soit $X$ une $\mathbb{Q}$-variété projective et lisse, géométriquement rationnelle. Pour presque tout nombre premier $p$, il existe une réduction $X_{p}$ de $X$ modulo $p$ qui est une $\mathbb{F}_{p}$-variété projective et lisse, géométriquement rationnelle, telle que

$$
H_{\mathrm{nr}}^{3}\left(X_{p}, \mathbb{Q}_{l} / \mathbb{Z}_{l}(2)\right) \stackrel{\simeq}{\rightrightarrows} \operatorname{Coker}\left[C H^{2}\left(X_{p}\right) \rightarrow C H^{2}\left(\bar{X}_{p}\right)^{G}\right]\{l\}
$$

pour tout nombre premier $l,(l, p)=1$.

Remarque 2.2. Pour définir $X_{p}$ on choisit un modèle projectif et lisse $\mathscr{Q}$ de $X$ au-dessus d'un ouvert convenable $U \subset \operatorname{Spec} \mathbb{Z},(p) \in U$, et on pose $X_{p}=\mathscr{X} \otimes \mathbb{F}_{p}$. Cette construction dépend du modèle choisi.

Pour démontrer le théorème 2.1, on utilise le résultat suivant :

Théorème 2.3 [Kahn 1996, théorème 1 et corollaire p. 397, partie 1]. Soit $k$ un corps de caractéristique $p \geq 0$, de dimension cohomologique au plus 3. Soit $X$ une $k$-variété projective et lisse. Supposons que

(i) $K_{2} \bar{k} \stackrel{\sim}{\rightarrow} H^{0}\left(\bar{X}, \mathscr{K}_{2}\right)$ et

(ii) le groupe $H_{\mathrm{nr}}^{3}(\bar{X}, \mathbb{Q} / \mathbb{Z}(2))$ est nul, resp. de torsion p-primaire si car. $k>0$. Alors on a une suite exacte naturelle, resp. exacte à la p-torsion près si car. $k>0$

$$
\begin{aligned}
H^{1}\left(k, H^{1}\left(\bar{X}, \mathscr{K}_{2}\right)\right) & \rightarrow \operatorname{Coker}\left[H^{3}(k, \mathbb{Q} / \mathbb{Z}(2)) \rightarrow H_{\mathrm{nr}}^{3}(X, \mathbb{Q} / \mathbb{Z}(2))\right] \rightarrow \\
& \rightarrow \operatorname{Coker}\left[C H^{2}(X) \rightarrow C H^{2}(\bar{X})^{G}\right] \rightarrow H^{2}\left(k, H^{1}\left(\bar{X}, \mathcal{K}_{2}\right)\right) .
\end{aligned}
$$

Remarque 2.4. Voir [Kahn 1996, p. 398] pour la définition des groupes de cohomologie à coefficients dans $\mathbb{Q} / \mathbb{Z}(2)$ en caractéristique positive.

Il est ainsi nécessaire de vérifier les hypothèses (i) et (ii) pour une variété géométriquement rationnelle $X$. Les énoncés suivants, cas particuliers de [Colliot-Thélène 1995], 2.1.9 (cf. aussi 4.1.5), sont bien connus.

Proposition 2.5. Soit $k$ un corps. Soit X une $k$-variété projective et lisse, $k$-rationnelle. Alors

(i) $H_{\mathrm{nr}}^{j}\left(X, \mu_{n}^{\otimes i}\right) \simeq H^{j}\left(k, \mu_{n}^{\otimes i}\right)$ pour tout $j \geq 1$;

(ii) l'application naturelle $K_{2} k \rightarrow H^{0}\left(X, \mathscr{K}_{2}\right)$ est un isomorphisme;

(iii) le groupe $\operatorname{Pic}(X)$ est libre de type fini. 
L'énoncé suivant permet de comprendre le module galoisien $H^{1}\left(\bar{X}, \mathscr{K}_{2}\right)$.

Proposition 2.6. Soit $k$ un corps algébriquement clos de caractéristique nulle. Le noyau $K(X)$ et le conoyau $C(X)$ de l'application $\operatorname{Pic}(X) \otimes k^{*} \rightarrow H^{1}\left(X, \mathscr{K}_{2}\right)$ sont des invariants birationnels des $k$-variétés intègres, projectives et lisses. En particulier, l'application $\operatorname{Pic}(X) \otimes k^{*} \rightarrow H^{1}\left(X, \mathscr{K}_{2}\right)$ est un isomorphisme pour $X$ une variété projective et lisse, $k$-rationnelle.

Démonstration. Le complexe de groupes abéliens

$$
\operatorname{Pic}(X) \otimes k^{*} \rightarrow H^{1}\left(X, K_{2}\right) .
$$

est fonctoriel contravariant pour les morphismes dominants de variétés projectives et lisses. Le noyau $K(X)$ et le conoyau $C(X)$ de $\operatorname{Pic}(X) \otimes k^{*} \rightarrow H^{1}\left(X, K_{2}\right)$ sont alors des foncteurs contravariants pour de tels morphismes. Soit $F$ l'un de ces foncteurs.

Soient $X, Y$ deux variétés intègres, projectives et lisses. Montrons qu'un morphisme birationnel $X \rightarrow Y$ induit un isomorphisme $F(Y) \rightarrow F(X)$. D'après Hironaka, il existe deux $k$-variétés projectives et lisses $X^{\prime}$ et $Y^{\prime}$ et un diagramme commutatif

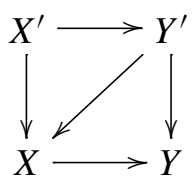

où les flèches verticales sont des suites d'éclatements de centres lisses. D'après le lemme ci-dessous, $F(X)$ est isomorphe à $F\left(X^{\prime}\right)$, respectivement $F(Y)$ est isomorphe à $F\left(Y^{\prime}\right)$. On en déduit par fonctorialité que $F(X)$ est isomorphe à $F(Y)$.

Si maintenant on a une application rationnelle $X \rightarrow Y$, on utilise Hironaka pour trouver une variété projective et lisse $Z$ avec $Z \rightarrow X$ et $Z \rightarrow Y$ deux morphismes birationnels. D'après ce qui précède, $F(X) \simeq F(Z) \simeq F(Y)$. Ainsi $F(X)$ est un invariant birationnel des $k$-variétés intègres, projectives et lisses.

Le fait que $F\left(\mathbb{P}_{k}^{n}\right)=0$ est bien connu. On établit d'abord que $H^{1}\left(\mathbb{A}_{k}^{1}, \mathscr{K}_{2}\right)=0$ et ensuite que $H^{1}\left(\mathbb{A}_{k}^{n}, \mathscr{K}_{2}\right)=0$ par des fibrations successives à fibres $\mathbb{A}^{1}$. L'énoncé pour $\mathbb{P}_{k}^{n}$ s'en suit par récurrence, en se restreignant à l'hyperplan à l'infini.

Remarque 2.7. On peut montrer plus généralement que pour $X$ lisse sur un corps, $H^{i}\left(\mathbb{A}_{X}^{n}, \mathscr{K}_{j}\right)$ est isomorphe à $H^{i}\left(X, \mathscr{K}_{j}\right)$, et donner une expression explicite de $H^{i}\left(\mathbb{P}_{X}^{n}, \mathscr{K}_{j}\right)$ en termes de $K$-cohomologie de $X$. Voir [Sherman 1979].

Lemme 2.8. Soit $k$ un corps algébriquement clos. Soit $X$ une $k$-variété intègre, projective et lisse. Soit $Z \subset X$ une sous-variété intègre, projective et lisse, de codimension au moins 2 et soit $\pi: X^{\prime} \rightarrow X$ l'éclatement de $X$ le long de $Z$. Alors les applications $K(X) \rightarrow K\left(X^{\prime}\right)$ et $C(X) \rightarrow C\left(X^{\prime}\right)$ sont des isomorphismes. 
Démonstration. Soit $Z^{\prime}$ le diviseur exceptionnel de $X^{\prime}$ et soit $U=X \backslash Z \simeq X^{\prime} \backslash Z^{\prime}$. Supposons d'abord que $Z$ est de codimension 2. On a les suites exactes horizontales de complexes verticaux :

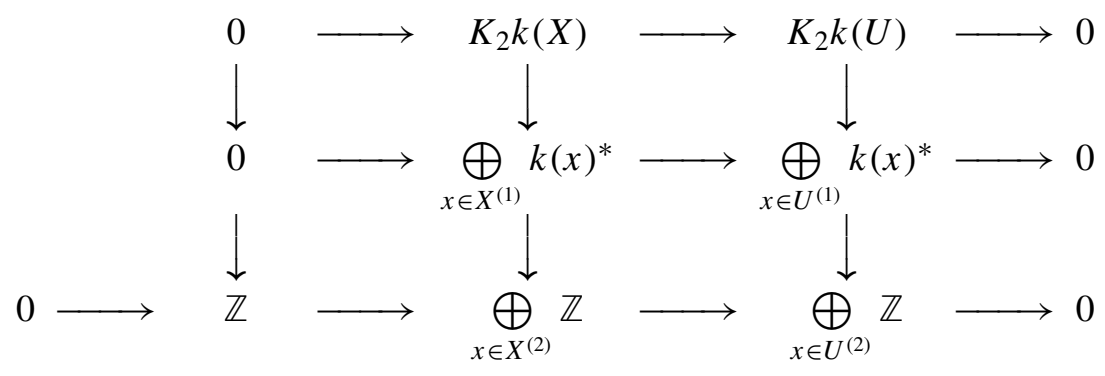

et

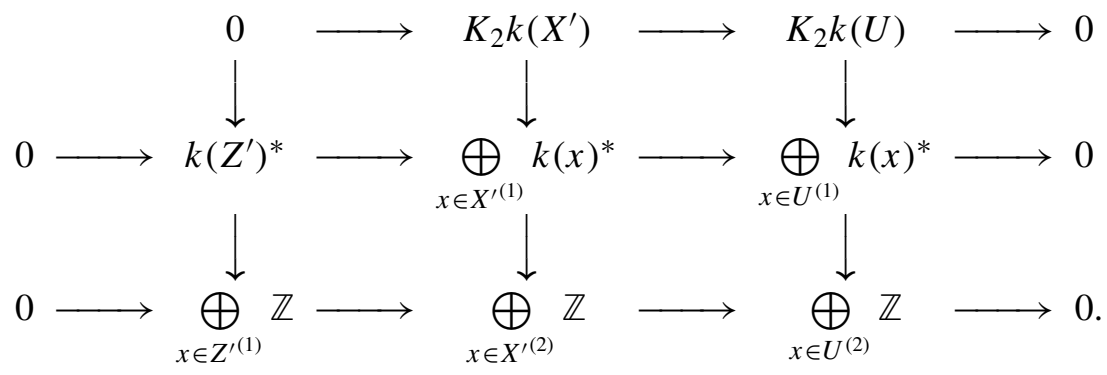

On a ainsi des suites longues induites en cohomologie :

$$
\begin{array}{r}
0 \rightarrow H^{0}\left(X, \mathscr{K}_{2}\right) \rightarrow H^{0}\left(U, \mathscr{K}_{2}\right) \rightarrow 0 \rightarrow H^{1}\left(X, \mathscr{K}_{2}\right) \rightarrow H^{1}\left(U, \mathscr{K}_{2}\right) \rightarrow \mathbb{Z} \rightarrow \\
\rightarrow C H^{2}(X) \rightarrow C H^{2}(U) \rightarrow 0, \quad \text { (3) } \\
0 \rightarrow H^{0}\left(X^{\prime}, \mathscr{K}_{2}\right) \rightarrow H^{0}\left(U, \mathscr{K}_{2}\right) \rightarrow k^{*} \rightarrow H^{1}\left(X^{\prime}, \mathscr{K}_{2}\right) \rightarrow H^{1}\left(U, \mathscr{K}_{2}\right) \rightarrow \operatorname{Pic}\left(Z^{\prime}\right) \rightarrow \\
\rightarrow C H^{2}\left(X^{\prime}\right) \rightarrow C H^{2}(U) \rightarrow 0 .
\end{array}
$$

Dans la suite (3), la flèche $\mathbb{Z} \rightarrow C H^{2}(X)$ est donnée par $1 \mapsto[Z]$. En prenant l'intersection avec un hyperplan général, on voit que cette flèche est injective. Ainsi l'application $H^{1}\left(X, \mathscr{K}_{2}\right) \rightarrow H^{1}\left(U, \mathscr{K}_{2}\right)$ est un isomorphisme.

Si $Z$ est de codimension plus grande que 2, on a encore la suite (4) et les groupes $H^{1}\left(X, \mathscr{K}_{2}\right)$ et $H^{1}\left(U, \mathscr{K}_{2}\right)$ sont isomorphes, car ils ne dependent que de points de codimension au plus 2 .

Par fonctorialité, on a le diagramme commutatif suivant :

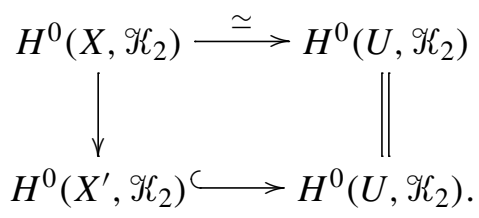


Ainsi l'application $H^{0}\left(X^{\prime}, \mathscr{K}_{2}\right) \rightarrow H^{0}\left(U, \mathscr{K}_{2}\right)$ est un isomorphisme. En utilisant la suite (4), on obtient le diagramme commutatif de suites exactes suivant :

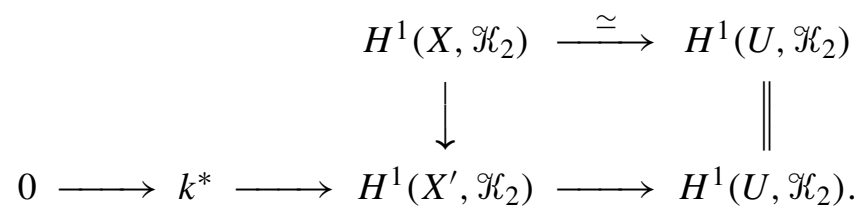

On a donc une suite exacte scindée :

$$
0 \rightarrow k^{*} \rightarrow H^{1}\left(X^{\prime}, \mathscr{K}_{2}\right) \rightarrow H^{1}\left(X, \mathscr{K}_{2}\right) \rightarrow 0 .
$$

Ainsi $H^{1}\left(X^{\prime}, \mathscr{K}_{2}\right) \simeq H^{1}\left(X, \mathscr{K}_{2}\right) \oplus k^{*}$. Puisque $\operatorname{Pic}\left(X^{\prime}\right)=\operatorname{Pic}(X) \oplus \mathbb{Z} \cdot\left[Z^{\prime}\right]$, on en déduit l'énoncé du lemme.

Remarque 2.9. En utilisant l'action des correspondances sur les groupes de Chow supérieurs, on peut établir la proposition 2.6 en toute caractéristique. Cela n'est pas nécessaire pour la démonstration du théorème 2.1 .

Preuve du théorème 2.1. Puisque $X$ est une $\mathbb{Q}$-variété géométriquement rationnelle, il existe une extension finie $K / \mathbb{Q}$ et une $K$-variété projective et lisse $Z$, deux morphismes $K$-birationnels $Z \rightarrow X_{K}$ et $Z \rightarrow \mathbb{P}_{K}^{n}$, et deux diagrammes commutatifs

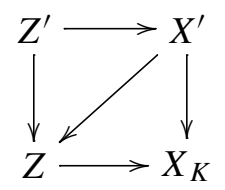

et

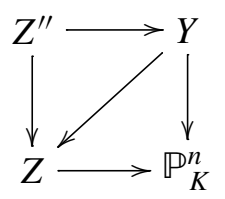

où les flèches verticales sont des suites d'éclatements de centres lisses. Pour presque toute place $v$ de $K$, les centres d'éclatements admettent des réductions lisses et les diagrammes (5) induisent des diagrammes analogues sur le corps résiduel $k(v)$. Ainsi, pour presque toute place $v$ de $K$, on peut définir une réduction $X_{p}$ de $X$ modulo $p, p=$ car. $k(v)$, qui est une $\mathbb{F}_{p}$-variété projective et lisse, géométriquement rationnelle et des réductions de $Z, Z^{\prime}, Z^{\prime \prime}, X$ et $Y$ sur $k(v)$, qui sont des variétés lisses et telles qu'on a des diagrammes commutatifs sur $\overline{\mathbb{F}}_{p}$
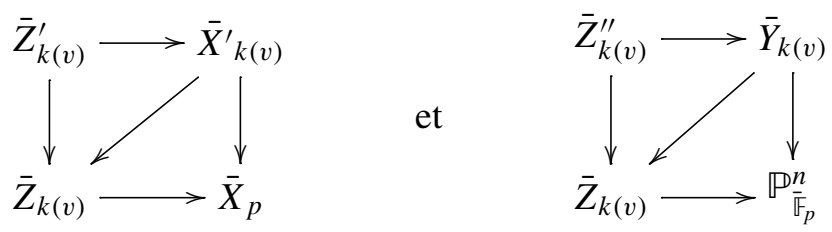

où les flèches verticales sont des suites d'éclatements de centres lisses. En appliquant le lemme 2.8, on déduit que l'application $\operatorname{Pic}\left(\bar{X}_{p}\right) \otimes \overline{\mathbb{F}}_{p}^{*} \rightarrow H^{1}\left(\bar{X}_{p}, \mathscr{K}_{2}\right)$ est un isomorphisme (voir aussi la proposition 2.6). 
Montrons que les hypothèses du théorème 2.3 sont satisfaites pour une telle réduction $X_{p}$. D'après la proposition $2.5, K_{2} \overline{\mathbb{F}}_{p} \stackrel{\sim}{\rightarrow} H^{0}\left(\bar{X}_{p}, \mathscr{K}_{2}\right)=0$ car $X_{p}$ est géométriquement rationnelle. De même, $H_{\mathrm{nr}}^{3}\left(\bar{X}_{p}, \mathbb{Q}_{l} / \mathbb{Z}_{l}(2)\right)=H^{3}\left(\overline{\mathbb{F}}_{p}, \mathbb{Q}_{l} / \mathbb{Z}_{l}(2)\right)=$ 0 car $\overline{\mathbb{F}}_{p}$ est séparablement clos.

Montrons ensuite que le groupe $H^{i}\left(\mathbb{F}_{p}, H^{1}\left(\bar{X}_{p}, \mathscr{K}_{2}\right)\right) \simeq H^{i}\left(\mathbb{F}_{p}, \operatorname{Pic}\left(\bar{X}_{p}\right) \otimes \overline{\mathbb{F}}_{p}^{*}\right)$ est nul pour tout $i \geq 1$. D'après la proposition 2.5, le $\mathbb{Z}$-module $\operatorname{Pic}\left(\bar{X}_{p}\right)$ est libre de type fini. Considérons une extension finie galoisienne $L / \mathbb{F}_{p}$ qui déploie $\operatorname{Pic}\left(\bar{X}_{p}\right)$. Considérons la suite de restriction-inflation :

$0 \rightarrow H^{1}\left(\operatorname{Gal}\left(L / \mathbb{F}_{p}\right), \operatorname{Pic} \bar{X}_{p, L} \otimes L^{*}\right) \rightarrow$

$$
H^{1}\left(\mathbb{F}_{p}, \operatorname{Pic} \bar{X}_{p} \otimes \mathbb{F}_{p}^{*}\right) \rightarrow H^{1}\left(\operatorname{Gal}\left(\overline{\mathbb{F}}_{p} / L\right), \operatorname{Pic} \bar{X}_{p} \otimes \overline{\mathbb{F}}_{p}^{*}\right) .
$$

On a $H^{1}\left(\operatorname{Gal}\left(\overline{\mathbb{F}}_{p} / L\right), \operatorname{Pic} \bar{X}_{p} \otimes \overline{\mathbb{F}}_{p}^{*}\right)=0$ d'après le théorème de Hilbert 90 . Puisque la dimension cohomologique de $\mathbb{F}_{p}$ est $1, H^{1}\left(\operatorname{Gal}\left(L / \mathbb{F}_{p}\right), \operatorname{Pic} X_{p, L} \otimes L^{*}\right)=0$ [Serre 1968, p. 170]. On a donc $H^{i}\left(\mathbb{F}_{p}, \operatorname{Pic}\left(\bar{X}_{p}\right) \otimes \overline{\mathbb{F}}_{p}^{*}\right)=0$ pour tout $i \geq 1$.

Notons que $H^{3}\left(\mathbb{F}_{p}, \mathbb{Q}_{l} / \mathbb{Z}_{l}(2)\right)=0$ car la dimension cohomologique de $\mathbb{F}_{p}$ est 1 . La suite (2) donne alors un isomorphisme :

$$
H_{\mathrm{nr}}^{3}\left(X_{p}, \mathbb{Q}_{l} / \mathbb{Z}_{l}(2)\right) \stackrel{\sim}{\rightarrow} \operatorname{Coker}\left[C H^{2}\left(X_{p}\right) \rightarrow C H^{2}\left(\bar{X}_{p}\right)^{G}\right]\{l\} .
$$

Remarque 2.10. Pour établir $H^{i}\left(k, H^{1}\left(\bar{X}, \mathscr{K}_{2}\right)\right)=0, i=1,2$ pour $X$ une variété projective et lisse, géométriquement rationnelle, définie sur un corps fini $k$, on aurait pu faire appel à des résultats généraux sur les variétés projectives et lisses [Colliot-Thélène et Raskind 1985, 2.12 et 2.14; Gros et Suwa 1988, 4.1]. Ces résultats généraux réposent en particulier sur les conjectures de Weil (démontrées par Deligne). Pour ce dont on a besoin dans la suite, le théorème 2.1 suffit.

\section{L'exemple}

Dans cette partie, pour une infinité de nombres premiers $p$, on construit une variété projective et lisse géométriquement rationnelle $X$, définie sur le corps fini $\mathbb{F}_{p}$, telle que l'application

$$
C H^{2}(X) \rightarrow C H^{2}(\bar{X})^{G}
$$

n'est pas surjective.

Remarque 3.1. Si $X$ est une variété projective et lisse, géométriquement intègre, définie sur le corps fini $\mathbb{F}_{p}$, l'application $\mathrm{CH}^{i}(X) \rightarrow C H^{i}(\bar{X})^{G}$ est surjective pour $i=0,1, d$. Le cas $i=0$ est immédiat. Pour $i=1, \operatorname{Pic}(X) \stackrel{\sim}{\rightarrow} C H^{1}(X)$ car $X$ est lisse. Puisque $X$ est projective et géométriquement intègre, $\bar{k}[X]^{*}=\bar{k}^{*}$ et la suite spectrale $E_{2}^{p q}=H^{p}\left(G, H^{q}\left(\bar{X}, \mathbb{G}_{m}\right)\right) \Rightarrow H^{p+q}\left(X, \mathbb{G}_{m}\right)$ donne une suite exacte

$$
0 \rightarrow \operatorname{Pic}(X) \rightarrow \operatorname{Pic}(\bar{X})^{G} \rightarrow H^{2}\left(G, \bar{k}^{*}\right) \rightarrow \operatorname{Br} X .
$$


Puisque le groupe $H^{2}\left(G, \bar{k}^{*}\right)=\operatorname{Br} k$ est nul pour un corps fini, on a la surjectivité pour $i=1$. Plus généralement, il en est ainsi pour toute variété $X$ projective et lisse, géométriquement intègre, avec un point rationnel, définie sur un corps $k$ quelconque : pour une telle variété l'application $H^{2}\left(G, \bar{k}^{*}\right) \rightarrow \operatorname{Br} X$ est injective.

Pour $i=d$, c'est-à-dire dans le cas de zéro-cycles, on sait que $X$ possède un zéro-cycle de degré 1 d'après les estimations de Lang et Weil [1954]. Il suffit donc de voir que l'application entre les groupes de Chow de zéro-cycles de degré zéro $A_{0}(X) \rightarrow A_{0}(\bar{X})^{G}$ est surjective. Ceci résulte de la comparaison de ces derniers groupes avec les points rationnels (resp. les $\overline{\mathbb{F}}_{p}$-points) de la variété d'Albanese $\operatorname{Alb}_{X}$ de $X$. En effet, l'application $A_{0}(X) \rightarrow \operatorname{Alb}_{X}\left(\mathbb{F}_{p}\right)$ est surjective [Kato et Saito 1983, proposition 9, p. 274], et l'application $A_{0}(\bar{X}) \rightarrow \operatorname{Alb}_{X}\left(\overline{\mathbb{F}}_{p}\right)$ est un isomorphisme [Rojtman 1980; Milne 1982].

D'après le théorème 2.1 , si $X$ est géométriquement rationnelle, il suffit d'assurer que le groupe $H_{\mathrm{nr}}^{3}\left(X, \mathbb{Q}_{l} / \mathbb{Z}_{l}(2)\right)$ est non nul pour un certain nombre premier $l$, $l \neq p$. Colliot-Thélène et Ojanguren [1989] construisent de tels exemples sur le corps des complexes pour $l=2$. Les variétés ainsi construites sont unirationnelles (c'est-à-dire, dominées par un ouvert de l'espace projectif). Via la proposition 2.5, on obtient ainsi des exemples de variétés unirationnelles non rationnelles. Dans la suite, on utilise la même méthode pour produire des exemples sur les corps finis.

La stratégie est la suivante :

(1) On considère une quadrique projective et lisse $Q$ sur le corps $F=\mathbb{F}_{p}(x, y)$, $p \neq 2$, définie dans $\mathbb{P}_{F}^{4}$ par une équation homogène

$$
x_{0}^{2}-a x_{1}^{2}-f x_{2}^{2}+a f x_{3}^{2}-g_{1} g_{2} x_{4}^{2}=0
$$

où $a \in \mathbb{F}_{p}$ est une constante et $f, g_{1}, g_{2} \in F$. La quadrique $Q$ admet un point rationnel sur $\overline{\mathbb{F}}_{p}(x, y)$, elle est donc $\overline{\mathbb{F}}_{p}(x, y)$-rationnelle.

(2) On donne des conditions nécessaires sur les coefficients dans (6) pour que le cup-produit $\left(a, f, g_{1}\right)$ soit non nul dans $H_{\mathrm{nr}}^{3}(Q, \mathbb{Z} / 2)$.

(3) On vérifie que l'on peut trouver $a \in \mathbb{Z}$ et $f, g_{1}, g_{2} \in \mathbb{Z}(x, y)$ tels que leurs réductions modulo $p$ vérifient les conditions de l'étape précédente pour le corps $\mathbb{F}_{p}$ pour une infinité de nombres premiers $p$. Par Hironaka, on trouve une variété projective et lisse $X$ définie sur $\mathbb{Q}$, admettant une fibration sur $\mathbb{P}_{\mathbb{Q}}^{2}$ de fibre générique la quadrique définie par (6). Pour presque tout $p, X$ admet une réduction $X_{p}$ modulo $p$ qui est lisse sur $\mathbb{F}_{p}$ et pour une infinité de premiers $p$ le groupe $H_{\mathrm{nr}}^{3}\left(X_{p}, \mathbb{Z} / 2\right)$ est ainsi non nul.

Cohomologie des quadriques. Soit $k$ un corps, car. $k \neq 2$. Soit $\phi$ une forme quadratique non dégénérée de dimension $m$ définie sur $k$. On note $X_{\phi}$ la quadrique projective et lisse dans $\mathbb{P}_{k}^{m-1}$ définie par $\phi$. On appelle $n$-forme de Pfister sur $k$ 
une forme quadratique de type $\left\langle 1,-a_{1}\right\rangle \otimes \cdots \otimes\left\langle 1,-a_{n}\right\rangle, a_{i} \in k^{*}$. Une forme quadratique non dégénérée $\phi$ est dite «voisine de Pfister» s'il existe une forme de Pfister $\phi^{\prime}$ sur $k$ et $a \in k^{*}$ tels que $\phi$ soit une sous-forme de $a \phi^{\prime}$ et que la dimension de $\phi$ soit strictement supérieure à la moitié de la dimension de $\phi^{\prime}$.

Théorème 3.2 [Arason 1975]. Soit $k$ un corps, car. $k \neq 2$. Soit $\phi$ une forme quadratique définie sur $k$, voisine d'une 3-forme de Pfister $\left\langle 1,-a_{1}\right\rangle \otimes\left\langle 1,-a_{2}\right\rangle \otimes\left\langle 1,-a_{3}\right\rangle$. Alors

$$
\operatorname{ker}\left[H^{3}(k, \mathbb{Z} / 2) \rightarrow H^{3}\left(k\left(X_{\phi}\right), \mathbb{Z} / 2\right)\right]=\mathbb{Z} / 2\left(a_{1}, a_{2}, a_{3}\right),
$$

chaque $a_{i}$ étant identifié à sa classe dans $H^{1}(k, \mathbb{Z} / 2) \simeq k^{*} / k^{* 2}$.

Soit $Q$ la quadrique définie sur le corps $F=\mathbb{F}_{p}(x, y), p \neq 2$, par l'équation homogène (6). D'après le théorème d'Arason,

$$
\operatorname{ker}\left[H^{3}(F, \mathbb{Z} / 2) \rightarrow H^{3}(F(Q), \mathbb{Z} / 2)\right]=\mathbb{Z} / 2\left(a, f, g_{1} g_{2}\right) .
$$

Pour trouver un élément non nul dans $H_{\mathrm{nr}}^{3}(Q, \mathbb{Z} / 2)$, on peut ainsi essayer de chercher un élément de $H^{3}(F, \mathbb{Z} / 2)$, différent de $\left(a, f, g_{1} g_{2}\right)$ et qui devient non ramifié dans $H^{3}(F(Q), \mathbb{Z} / 2)$. On va choisir les éléments $a, f, g_{1}$ et $g_{2}$ pour que l'élément $\left(a, f, g_{1}\right)$ convienne.

Faisons d'abord quelques rappels sur les calculs de résidus.

Proposition 3.3 [Colliot-Thélène et Ojanguren 1989, 1.3 et 1.4]. Soit A un anneau de valuation discrète, de corps des fractions $K$ et de corps résiduel $k$. Soit $j \geq 1$ un entier.

(1) Soit $\alpha \in H^{j}(A, \mathbb{Z} / 2)$ et soit $\alpha_{0} \in H^{j}(k, \mathbb{Z} / 2)$ son image par l'application de réduction. Soit $b \in K^{*}$ de valuation $m$ dans $A$ et soit $\beta$ la classe de $b$ dans $H^{1}(K, \mathbb{Z} / 2)$. Alors $\partial_{A}(\alpha \cup \beta)=m \alpha_{0}$.

(2) Soit $\alpha \in H^{j}(K, \mathbb{Z} / 2)$ et soit $b \in A^{*}$ dont la classe est un carré dans $k$. Soit $\beta$ la classe de $b$ dans $H^{1}(K, \mathbb{Z} / 2)$. Alors $\partial_{A}(\alpha \cup \beta)=0$.

On décrit ensuite les conditions qu'on va imposer sur les coefficients de la quadrique $Q$ :

Proposition 3.4. Soit $F=\mathbb{F}_{p}(x, y)$ le corps des fractions rationnelles à deux variables sur le corps fini $\mathbb{F}_{p}$. Soit $a \in \mathbb{F}_{p}^{*} \backslash \mathbb{F}_{p}^{* 2}$ et soient $f, g_{1}, g_{2} \in F$ non nuls. Soit $Q$ la quadrique lisse dans $\mathbb{P}_{F}^{4}$ d'équation homogène

$$
x_{0}^{2}-a x_{1}^{2}-f x_{2}^{2}+a f x_{3}^{2}-g_{1} g_{2} x_{4}^{2}=0 .
$$

\section{Supposons}

(1) pour tout $i=1,2$, il existe un anneau de valuation discrète $B_{i}$ de corps des fractions $F$, tel que $\partial_{B_{i}}\left(a, f, g_{i}\right) \neq 0$; 
(2) pour tout anneau de valuation discrète $B$ de corps des fractions $F$, associé à un point de codimension 1 de $\mathbb{P}_{\mathbb{F}_{p}}^{2}$, soit $\partial_{B}\left(a, f, g_{1}\right)=0$, soit $\partial_{B}\left(a, f, g_{2}\right)=0$;

(3) pour tout anneau de valuation discrète $B$ de corps des fractions $F$, centré en un point fermé $M$ de $\mathbb{P}_{\mathbb{F}_{p}}^{2}$, quitte à la multiplier par un carré dans $F^{*}$, l'une au moins des fonctions $f, g_{1}, g_{2}$ est inversible en $M$.

Alors l'image $\xi_{F(Q)} d u$ cup-produit $\xi=\left(a, f, g_{1}\right)$ dans $H^{3}(F(Q), \mathbb{Z} / 2)$ est un élément non nul de $H_{\mathrm{nr}}^{3}(Q, \mathbb{Z} / 2)$.

Démonstration. Notons d'abord que $\left(a, f, g_{1}\right)$ est non nul dans $H^{3}(F(Q), \mathbb{Z} / 2)$. Sinon, d'après le théorème 3.2 , on a soit $\left(a, f, g_{1}\right)=0$, soit $\left(a, f, g_{1}\right)=\left(a, f, g_{1} g_{2}\right)$. Ainsi soit $\left(a, f, g_{1}\right)=0$, soit $\left(a, f, g_{2}\right)=0$, contradiction avec la condition 1 .

Montrons que pour tout anneau de valuation discrète $B$ de $F$

$$
\text { soit } \partial_{B}\left(a, f, g_{1}\right)=0 \text {, soit } \partial_{B}\left(a, f, g_{2}\right)=0 \text {. }
$$

Pour un tel anneau $B$ on dispose d'un morphisme Spec $B \rightarrow \mathbb{P}_{\mathbb{F}_{p}}^{2}$ et les cas 2 et 3 correspondent à deux possibilités pour l'image du point fermé de $B$. La condition (2) assure $(*)$ si cette image est un point de codimension $1 \mathrm{de} \mathbb{P}_{\mathbb{F}_{p}}^{2}$. Sinon l'image du point $\operatorname{Spec} k_{B}$ est un point fermé $M$ de $\mathbb{P}_{\mathbb{F}_{p}}^{2}$. Soit $\bigcirc_{M}$ l'anneau local de $M$, son corps des fractions est $F$. On dispose d'un morphisme d'anneaux $\mathrm{O}_{M} \rightarrow B$.

On peut supposer, sans perte de généralité, que la fonction $g_{1}$ est inversible dans $\mathrm{O}_{M}$, quitte à la multiplier par un carré. Ainsi la fonction $g_{1}$ est inversible dans $B$. Soit $m$ la valuation de $f$ dans $B$. D'après la proposition 3.3.1, on a

$$
\partial_{B}\left(a, f, g_{1}\right)=\partial_{B}\left(g_{1}, a, f\right)=m\left(\bar{g}_{1}, \bar{a}\right),
$$

où l'on note $\bar{g}_{1}$ et $\bar{a}$ les classes de $g_{1}$ et de $a$ dans $H^{1}\left(k_{B}, \mathbb{Z} / 2\right)$. Comme $g_{1}$ et $a$ sont inversibles dans $\mathrm{O}_{M}$, ces dernières classes proviennent de classes dans $H^{1}\left(k_{M}, \mathbb{Z} / 2\right)$. Ainsi $\left(\bar{g}_{1}, \bar{a}\right)$ provient d'un élément de $H^{2}\left(k_{M}, \mathbb{Z} / 2\right)$. Ce dernier groupe est nul, car $k_{M}$, étant un corps fini, est de dimension cohomologique 1. Ainsi $\partial_{B}\left(a, f, g_{1}\right)=0$.

Montrons maintenant que $\xi_{F(Q)}$ est non ramifié. Soit $A$ un anneau de valuation discrète de $F(Q)$ de corps résiduel $k_{A}$. Si $A$ contient $F$, alors $\xi_{F(Q)}$ provient d'un élément de $H^{3}(A, \mathbb{Z} / 2)$ et son résidu est donc nul. Supposons que $A$ ne contient pas $F$. Alors $B=A \cap F$ est un anneau de valuation discrète de $F$. Soit $k_{B}$ son corps résiduel. On a le diagramme commutatif suivant [Colliot-Thélène et Ojanguren $1989, \S 1]$ :

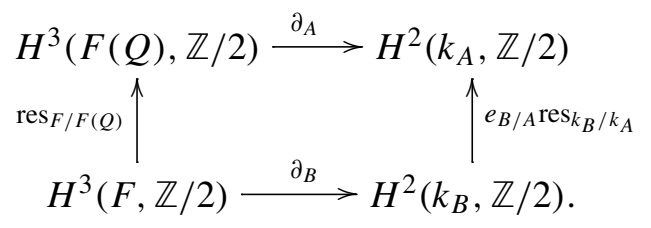


D'après ce qui précède, $\partial_{B}\left(a, f, g_{i}\right)=0$ pour $i=1$ ou pour $i=2$. Si $\partial_{B}\left(a, f, g_{1}\right)=0$, alors $\partial_{A}\left(a, f, g_{1}\right)=0$ d'après le diagramme (8). Supposons que $\partial_{B}\left(a, f, g_{2}\right)=0$. Ainsi $\partial_{A}\left(a, f, g_{2}\right)=0$. Comme $\left(a, f, g_{1} g_{2}\right)$ est nul dans $H^{3}(F(Q), \mathbb{Z} / 2)$, son résidu l'est aussi dans $H^{2}\left(k_{A}, \mathbb{Z} / 2\right)$. On a donc

$$
\partial_{A}\left(a, f, g_{1}\right)=\partial_{A}\left(a, f, g_{1} g_{2}\right)-\partial_{A}\left(a, f, g_{2}\right)=0 .
$$

Ainsi $\xi_{F(Q)}$ est non ramifié.

Construction explicite. On procède maintenant à la construction des exemples.

Soit $k$ un corps. Dans la suite, on va prendre $k=\mathbb{F}_{p}$ ou $k=\mathbb{Q}$. On fixe $x, y, z$ des coordonnées homogènes pour $\mathbb{P}_{k}^{2}$. Soit $a \in k^{*} \backslash k^{* 2}$. Soient $b_{i}, c_{i}, d_{i} \in k^{*} \backslash\{-1\}$, $i=1$, 2, et soit $l_{i}=b_{i} x+c_{i} y+d_{i} z$. Soient $h_{j}, j=1, \ldots, 8$, les formes linéaires $e_{x} x+e_{y} y+e_{z} z, e_{x}, e_{y}, e_{z} \in\{0,1\}$.

On choisit $b_{i}, c_{i}, d_{i}$ de sorte que :

(i) Les droites dans $\mathbb{P}_{k}^{2}$ données par les équations $x=0, y=0, z=0, l_{i}+h_{j}=0$, $i=1,2, j=1, \ldots, 8$, soient deux à deux distinctes.

(ii) Pour tous $1 \leq j, j^{\prime} \leq 8$ les trois droites $x=0, l_{1}+h_{j}=0, l_{2}+h_{j^{\prime}}=0$ dans $\mathbb{P}_{k}^{2}$ sont d'intersection vide.

(iii) Pour tous $1 \leq j, j^{\prime} \leq 8$ les trois droites $y=0, l_{1}+h_{j}=0, l_{2}+h_{j^{\prime}}=0$ dans $\mathbb{P}_{k}^{2}$ sont d'intersection vide.

On prend pour $f, g_{1}, g_{2} \in k\left(\mathbb{P}_{k}^{2}\right)$ les éléments suivants :

$$
f=\frac{x}{y}, \quad g_{1}=\frac{\prod_{j}\left(l_{1}+h_{j}\right)}{y^{8}}, \quad g_{2}=\frac{\prod_{j}\left(l_{2}+h_{j}\right)}{z^{8}} .
$$

Remarque 3.5. Soit $h_{j}=e_{x} x+e_{y} y+e_{z} z$. Les droites $x=0, l_{1}+h_{j}=0$ s'intersectent en un seul point $\left[0:\left(d_{1}+e_{z}\right):-\left(c_{1}+e_{y}\right)\right]$. Ainsi les conditions (ii) et (iii) ci-dessus sont équivalentes aux conditions suivantes :

(ii') Les ensembles

$\left\{\left[\left(d_{1}+e_{z}\right):-\left(c_{1}+e_{y}\right)\right], e_{y}, e_{z} \in\{0,1\}\right\}$ et $\left\{\left[\left(d_{2}+e_{z}\right):-\left(c_{2}+e_{y}\right)\right], e_{y}, e_{z} \in\{0,1\}\right\}$ sont d'intersection vide.

(iii') De même,

$\left\{\left[\left(d_{1}+e_{z}\right):-\left(b_{1}+e_{x}\right)\right], e_{x}, e_{z} \in\{0,1\}\right\} \cap\left\{\left[\left(d_{2}+e_{z}\right):-\left(b_{2}+e_{x}\right)\right], e_{x}, e_{z} \in\{0,1\}\right\}=\varnothing$.

Par exemple, pour

$$
l_{1}=x+y+2 z, l_{2}=3 x+3 y+z
$$

il s'agit de vérifier que les ensembles $\{[2:-1],[2:-2],[3:-1],[3:-2]\}$ et $\{[1:-3],[1:-4],[2:-3],[2:-4]\}$ sont d'intersection vide. Cette condition est satisfaite pour $k=\mathbb{Q}$ ou $k=\mathbb{F}_{p}$ un corps fini avec $p \geq 13$. 
Proposition 3.6. Soit $F=\mathbb{F}_{p}(x, y)$ le corps des fractions rationnelles à deux variables sur le corps fini $\mathbb{F}_{p}$. Soit $Q$ la quadrique lisse dans $\mathbb{P}_{F}^{4}$ d'équation homogène

$$
x_{0}^{2}-a x_{1}^{2}-f x_{2}^{2}+a f x_{3}^{2}-g_{1} g_{2} x_{4}^{2}=0
$$

avec $a \in \mathbb{F}_{p}^{*} \backslash \mathbb{F}_{p}^{* 2}$ et $f, g_{1}, g_{2}$ définis comme dans (9) pour $k=\mathbb{F}_{p}$. Alors le groupe $H_{\mathrm{nr}}^{3}(Q, \mathbb{Z} / 2)$ est non nul.

Démonstration. Notons $A_{x}\left(A_{y}, A_{z}, B_{i, j}\right.$, où $i=1,2$ et $\left.j=1, \ldots, 8\right)$ l'anneau de valuation discrète associé au point générique de la droite $x=0(y=0, z=0$, $\left.l_{i}+h_{j}=0\right)$.

Il s'agit de vérifier les conditions 1, 2 et 3 de la proposition 3.4. Soit $B$ un anneau de valuation discrète de $F$. On a les cas suivants à considérer :

$\underline{B}$ correspond à un point de codimension 1 de $\mathbb{P}_{\mathbb{F}_{p}}^{2}$.

(a) Si $B$ est différent de $A_{x}, A_{y}, A_{z}, B_{i, j}$, le résidu $\partial_{B}\left(a, f, g_{i}\right), i=1,2$, est nul, puisque les fonctions $a, f, g_{1}, g_{2}$ sont inversibles dans un tel anneau $B$.

(b) $B=B_{i, j}$. Si $r \neq i, r=1,2$, alors $\partial_{B_{r, j}}\left(a, f, g_{i}\right)=0$ comme le cas précédent. Fixons $i \in\{1,2\}$. Montrons que $\partial_{B_{i, j}}\left(a, f, g_{i}\right) \neq 0$. Supposons $h_{j}=0$, les autres cas sont identiques. Soit $k$ le corps résiduel de $B_{i, 0}$, i.e. le corps des fonctions de la droite $b_{i} x+c_{i} y+d_{i} z=0, b_{i}, c_{i}, d_{i} \in k^{*}$ (pour $h_{j}$ différent de zéro on utilise ainsi l'hypothèse que $b_{i}, c_{i}, d_{i}$ sont différents de -1$)$. D'après la proposition 3.3.1, $\partial_{B_{i, 0}}\left(a, f, g_{i}\right)=\left(a, \frac{x}{y}\right) \in H^{2}(k, \mathbb{Z} / 2)$. Après passage à des coordonnées affines, on est réduit à établir que le cup-produit $(a, x)$ n'est pas nul dans $H^{2}\left(\mathbb{F}_{p}(x), \mathbb{Z} / 2\right)$. On le voit par exemple en appliquant la proposition 3.3.1 à l'anneau de valuation discrète associé à $x=0: a \in \mathbb{F}_{p}$ est non carré.

(c) $B=A_{x}$. Montrons que $\partial_{A_{x}}\left(a, f, g_{i}\right)=0, i=1,2$. Soit $k_{x}$ le corps résiduel de $A_{x}$, i.e. le corps des fonctions de la droite $x=0$. D'après la proposition 3.3.1, $\left.\partial_{A_{x}}\left(a, f, g_{i}\right)=-\partial_{A_{x}}\left(a, g_{i}, f\right)=-\left(a, g_{i, x}\right) \in H^{2}\left(k_{x}, \mathbb{Z} / 2\right)\right)$, où $g_{i, x}$ désigne la fonction induite par $g_{i}$ sur la droite $x=0$. Mais $g_{i, x}$ est un carré dans $k_{x}$, d'où $\partial_{A_{x}}\left(a, f, g_{i}\right)=0$ d'après la proposition 3.3.2.

(d) $B=A_{y}$. Comme dans le cas précédent, $\partial_{A_{y}}\left(a, f, g_{2}\right)=\left(a, g_{2, y}\right)=0$.

(e) $B=A_{z}$. Alors $\partial_{A_{z}}\left(a, f, g_{1}\right)=0$, car les fonctions $a, f, g_{1}$ sont inversibles dans $A_{z}$.

B correspond à un point fermé $M$ de $\mathbb{P}_{\mathbb{F}_{p}}^{2}$.

(a) Si $M$ n'est pas situé sur une des deux droites $x=0, y=0$, alors $f$ est inversible dans $B$.

(b) Si $M$ est situé sur une des deux droites $x=0, y=0$, alors l'une au moins des fonctions $g_{1} \frac{y^{8}}{z^{8}}, g_{2}$ est inversible dans $B$ d'après les hypothèses (ii)-(iii), car le système $x y=0, \prod_{j}\left(l_{1}+h_{j}\right)=0, \prod_{j}\left(l_{2}+h_{j}\right)=0$ n'a pas de solutions. 
On finit par décrire explicitement les exemples énoncés.

Théorème 3.7. Soit $Q$ la quadrique lisse dans $\mathbb{P}_{\mathbb{Q}(x, y)}^{4}$ d'équation homogène

$$
x_{0}^{2}-a x_{1}^{2}-f x_{2}^{2}+a f x_{3}^{2}-g_{1} g_{2} x_{4}^{2}=0
$$

avec $a \in \mathbb{Q}^{*} \backslash \mathbb{Q}^{* 2}$ et $f, g_{1}, g_{2}$ définis comme dans $(9)$ pour $k=\mathbb{Q}$. Soit X un modèle projectif et lisse de $Q$ sur $\mathbb{P}_{\mathbb{Q}}^{2}: X$ est une $k$-variété projective et lisse et admet une fibration sur $\mathbb{P}_{\mathbb{Q}}^{2}$ à fibre générique $Q$. Pour une infinité de nombres premiers $p$, la réduction $X_{p}$ de $X$ modulo $p$ est bien définie et est une $\mathbb{F}_{p}$-variété projective et lisse, telle que:

(i) $H_{\mathrm{nr}}^{3}\left(X_{p}, \mathbb{Z} / 2\right) \neq 0$;

(ii) l'application $\mathrm{CH}^{2}(X) \rightarrow \mathrm{CH}^{2}(\bar{X})^{G}$ n'est pas surjective.

Démonstration. D'après Hironaka, un modèle projectif et lisse $X$ de $Q$ comme dans l'énoncé existe. De plus, pour une infinité de nombres premiers $p$, l'image de $a$ dans $\mathbb{F}_{p}$ n'est pas un carré (par Chebotarev, ou par application de la loi de réciprocité quadratique) et la variété $X$ a bonne réduction en $p: X_{p}$ est lisse. D'après la proposition 3.6, le groupe $H_{\mathrm{nr}}^{3}\left(X_{p}, \mathbb{Z} / 2\right)$ est non nul. Le théorème 2.1 permet de conclure.

\section{Remerciements}

Je tiens à exprimer ma profonde gratitude à mon directeur de thèse à l'Université Paris-Sud (Orsay), Jean-Louis Colliot-Thélène, pour m' avoir suggéré d'utiliser les méthodes de [Colliot-Thélène et Ojanguren 1989] et m'avoir introduite dans le sujet; sans ses nombreux conseils et réponses cet article n'aurait pas pu voir le jour.

\section{References}

[Arason 1975] J. K. Arason, "Cohomologische invarianten quadratischer Formen”, J. Algebra 36:3 (1975), 448-491. MR 52 \#10592 Zbl 0314.12104

[Colliot-Thélène 1995] J.-L. Colliot-Thélène, "Birational invariants, purity and the Gersten conjecture", pp. 1-64 dans $K$-theory and algebraic geometry: connections with quadratic forms and division algebras (Santa Barbara, CA, 1992), édité par B. Jacob et A. Rosenberg, Proc. Sympos. Pure Math. 58, Amer. Math. Soc., Providence, RI, 1995. MR 96c:14016 Zbl 0834.14009

[Colliot-Thélène et Ojanguren 1989] J.-L. Colliot-Thélène et M. Ojanguren, "Variétés unirationnelles non rationnelles: au-delà de l'exemple d'Artin et Mumford”, Invent. Math. 97:1 (1989), 141-158. MR 90m:14012

[Colliot-Thélène et Raskind 1985] J.-L. Colliot-Thélène et W. Raskind, " $\mathscr{Y}_{2}$-cohomology and the second Chow group”, Math. Ann. 270:2 (1985), 165-199. MR 86m:14005 Zbl 0536.14004 
[Gros et Suwa 1988] M. Gros et N. Suwa, "Application d'Abel-Jacobi p-adique et cycles algébriques”, Duke Math. J. 57:2 (1988), 579-613. MR 89h:14006a Zbl 0697.14005

[Kahn 1996] B. Kahn, "Applications of weight-two motivic cohomology", Doc. Math. 1:17 (1996), 395-416. MR 98b:14007 Zbl 0883.19002

[Kato et Saito 1983] K. Kato et S. Saito, "Unramified class field theory of arithmetical surfaces", Ann. of Math. (2) 118:2 (1983), 241-275. MR 86c:14006 Zbl 0562.14011

[Lang et Weil 1954] S. Lang et A. Weil, "Number of points of varieties in finite fields", Amer. J. Math. 76 (1954), 819-827. MR 16,398d Zbl 0058.27202

[Milne 1982] J. S. Milne, “Zero cycles on algebraic varieties in nonzero characteristic: Rojtman's theorem”, Compositio Math. 47:3 (1982), 271-287. MR 85b:14011 Zbl 0506.14006

[Quillen 1973] D. Quillen, "Higher algebraic $K$-theory, I", pp. 85-147 dans Algebraic K-theory, I: Higher K-theories (Seattle, 1972), édité par H. Bass, Lecture Notes in Math. 341, Springer, Berlin, 1973. MR 49 \#2895 Zbl 0292.18004

[Rojtman 1980] A. A. Rojtman, "The torsion of the group of 0-cycles modulo rational equivalence", Ann. of Math. (2) 111:3 (1980), 553-569. MR 81g:14003 Zbl 0504.14006

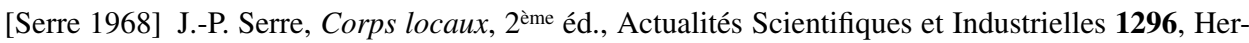
mann, Paris, 1968. MR 50 \#7096 Zbl 0137.02601

[Sherman 1979] C. C. Sherman, "K-cohomology of regular schemes", Comm. Algebra 7:10 (1979), 999-1027. MR 80h:14004 Zbl 0425.18010

Communicated by János Kollár

Received 2010-05-14 Revised 2010-10-12 Accepted 2010-11-15

alena.pirutka@ens.fr École Normale Supérieure, 45 rue d'Ulm, 75230 Paris, France 


\title{
Sur les invariants d'Iwasawa dans les extensions de Lie $p$-adiques
}

\author{
Guillaume Perbet
}

Soit $K_{\infty} / K$ une extension infinie du corps de nombres $K$, dont le groupe de Galois $G$ est un pro- $p$-groupe $p$-valué de type fini et dans laquelle seul un nombre fini de places sont ramifiées. La filtration entière de $G$ fixe des étages finis $K_{n} / K$ dans la tour $K_{\infty} / K$. Pour deux ensembles finis $S$ et $T$ de places de $K$, on attache à $K_{n}$ le groupe de Galois $X_{S, n}^{T}$ de sa pro-p-extension abélienne $S$-ramifiée et $T$ décomposée maximale, que la théorie du corps de classes interprète comme un groupe des classes généralisées. On démontre pour ces groupes des formules asymptotiques semblables à celle du théorème d'Iwasawa sur l'évolution du nombre de classes le long d'une $\mathbb{Z}_{p}$-extension. Les preuves utilisent les résultats de structure en théorie d'Iwasawa non-commutative et font apparaître les invariants d'Iwasawa attachés au module à l'infini $X_{S, \infty}^{T}$. Ces formules donnent des relations de dualité entre les invariants de $X_{S, \infty}^{T}$ et ceux de $X_{T, \infty}^{S}$, et débouchent sur des calculs d'invariants d'Iwasawa.

Let $K_{\infty} / K$ be an infinite extension of the number field $K$, with a $p$-valued pro- $p$-group of finite type $G$ as Galois group, and such that only finitely many primes are ramified in this extension. The integral filtration on $G$ fixes finite layers $K_{n} / K$ in the tower $K_{\infty} / K$. For $S$ and $T$ two finite sets of primes of $K$, one can attach to $K_{n}$ the Galois group $X_{S, n}^{T}$ of his maximal $S$-ramified and $T$-split abelian pro- $p$-extension, which can be viewed as a generalized class group via class field theory. For these groups, we prove asymptotic formulas similar to Iwasawa's theorem on class numbers in $\mathbb{Z}_{p}$-extensions. The proofs use structure results in noncommutative Iwasawa theory and involve Iwasawa's invariants of the module at infinity $X_{S, \infty}^{T}$. These formulas give duality relations between invariants of $X_{S, \infty}^{T}$ and those of $X_{T, \infty}^{S}$, and lead to computations of Iwasawa invariants.

MSC2000: primary 11R23; secondary 11R29.

Mots-clefs: théorie d'Iwasawa non-commutative, invariants d'Iwasawa, extensions de Lie $p$-adiques, formules asymptotiques, noncommutative Iwasawa theory, Iwasawa invariants, $p$-adic Lie extensions, asymptotic formulas. 


\section{Introduction}

Soit $p$ un nombre premier et soit $K$ un corps de nombres. Soit $K_{\infty} / K$ une $\mathbb{Z}_{p}$-extension, de groupe de Galois $\Gamma$. Dans les articles fondateurs de la théorie d'Iwasawa se trouve une formule asymptotique qui donne l'évolution du cardinal de la $p$-partie du groupe des classes le long de $K_{\infty} / K$. Elle est donnée dans le théorème suivant, où $X_{n}$ désigne la $p$-partie du groupe des classes du $n$-ième étage $K_{n}$ de la tour $K_{\infty} / K$ :

Théorème (Iwasawa [Serre 1966, théorème 2]). Il existe des entiers $\mu, \lambda \in \mathbb{N}$ et $v \in \mathbb{Z}$ ainsi qu'un rang $n_{0}$ tels que pour $n \geqslant n_{0}$

$$
\# X_{n}=p^{\mu p^{n}+\lambda_{n}+v} .
$$

La démonstration de ce résultat est évocatrice de l'efficacité des techniques de montée-descente, et du rôle central que joue l'algèbre d'Iwasawa $\Lambda(\Gamma)=\mathbb{Z}_{p} \llbracket \Gamma \rrbracket$. En effet, les constantes $\mu$ et $\lambda$ sont des invariants algébriques attachés au $\Lambda$-module à l'infini $X_{\infty}$, limite projective pour les applications norme des $X_{n}$. Ils proviennent d'un théorème de structure à pseudo-isomorphisme près pour les $\Lambda(\Gamma)$-modules de type fini (voir [Serre 1966]). Ce résultat a été étendu à des $\mathbb{Z}_{p}^{d}$ extensions par Cuoco et Monsky [1981].

Pour $S$ et $T$ deux ensembles finis disjoints de places de $K$, le théorème d'Iwasawa fut généralisé aux $p$-groupes des $T$-classes $S$-infinitésimales $X_{S, n}^{T}$ des corps $K_{n}$ par Jaulent [1986; 2005]. La principale différence avec le groupe des classes classique vient du fait que $X_{S, n}^{T}$ n'est pas en général fini, ce qui entraîne l'apparition d'un invariant supplémentaire $\rho$. Il démontre :

Théorème [Jaulent 2005]. Il existe des entiers $\rho_{S}^{T}$ et $\mu_{S}^{T}$ tels que

$$
\#\left(X_{S, n}^{T} / p^{n}\right)=p^{\rho_{S}^{T} n p^{n}+\mu_{S}^{T} p^{n}+O(n)} .
$$

Plus récemment, de nombreux auteurs se sont penchés sur une version noncommutative de la théorie d'Iwasawa. Les extensions infinies $K_{\infty} / K$ que l'on considère ont pour groupe de Galois un groupe de Lie $p$-adique $G$. Comme en théorie commutative, il est essentiel de bien connaître la structure des modules de type fini sur l'algèbre d'Iwasawa $\Lambda=\mathbb{Z}_{p} \llbracket G \rrbracket$. Même si l'on ne dispose pas d'un théorème de structure satisfaisant, des résultats partiels ont été obtenus par Venjakob [2002] et par Coates, Schneider et Sujatha [Coates et al. 2003]. Dans leurs travaux, ils introduisent une bonne notion de pseudo-nullité dans le cadre non-commutatif et ils définissent les invariants d'Iwasawa $\rho$ et $\mu$ d'un $\Lambda$-module de type fini. On ajoute ici un troisième invariant, noté $r$, qui tout comme $\mu$ est attaché à la partie de $\mathbb{Z}_{p}$-torsion du $\Lambda$-module étudié (définition 1.4).

On propose, dans ce travail, de généraliser les formules asymptotiques sur le nombre de classes lorsque l'on monte dans une extension de Lie $p$-adique $K_{\infty} / K$ 
dont le groupe de Galois $G$ est un pro- $p$-groupe $p$-valué de type fini de dimension $d \geqslant 1$, et dans laquelle seul un nombre fini de places de $K$ se ramifient.

On introduit la filtration entière $G_{n}$ sur $G$, qui est une suite de sous-groupes d'indice $C p^{n d}$ pour $n$ assez grand. On détermine alors l'évolution asymptotique des $p$-groupes des classes généralisées $X_{S, n}^{T}$ attachés aux sous-corps $K_{n}$, fixés par $G_{n}$.

Pour ce faire, on considère le $\Lambda$-module à l'infini $X_{S, \infty}^{T}=\lim _{\leftarrow} X_{S, n}^{T}$. Il est possible de redescendre à partir de ce module pour obtenir des informations sur les groupes $X_{S, n}^{T}$ (proposition 3.2). Notons $\rho_{S}^{T}, \mu_{S}^{T}, r_{S}^{T} \in \mathbb{N}$ les invariants d'Iwasawa de $X_{S, \infty}^{T}$. Cette technique de descente permet déjà de voir, utilisant un résultat de Harris, que l'invariant $\rho_{S}^{T}$ contrôle l'évolution asymptotique du $\mathbb{Z}_{p}$-rang des $X_{S, n}^{T}$ :

Théorème [Harris 2000, théorème 1.10].

$$
\operatorname{rk}_{\mathbb{Z}_{p}}\left(X_{S, n}^{T}\right)=\rho_{S}^{T}\left(G: G_{n}\right)+O\left(p^{n(d-1)}\right) .
$$

Cette formule conduit au calcul de l'invariant $\rho_{S}^{T}$ lorsque $K_{\infty} / K$ contient la $\mathbb{Z}_{p}$-extension cyclotomique de $K$ et que les étages sont totalements réels ou à multiplication complexe $(\mathrm{CM})$ : voir théorème 3.14.

Pour dégager des résultats plus précis, faisant intervenir les invariants $\mu$ et $r$, on effectue une étude algébrique approfondie des $\Lambda$-modules. On arrive alors au résultat suivant :

Théorème 0.1. On $a$

$$
\operatorname{dim}_{\mathbb{F}_{p}}\left(X_{S, n}^{T} / p\right)=\left(\rho_{S}^{T}+r_{S}^{T}\right)\left(G: G_{n}\right)+O\left(p^{n(d-1)}\right)
$$

et

$$
\#\left(X_{S, n}^{T} / p^{n}\right)=p^{\left(\rho_{S}^{T} n+\mu_{S}^{T}\right)\left(G: G_{n}\right)+O\left(n p^{n(d-1)}\right)} .
$$

A l'image de ce qui est fait par Jaulent et Maire [2003] dans le cas commutatif, ces résultats sont exploités à l'aide des formules de réflexion de Gras [1998]. Lorsque l'ensemble $S \cup T$ contient les places $p$-adiques $\mathrm{Pl}_{p}$ et en présence de racines de l'unité, on obtient une dualité entre décomposition et ramification qui prend la forme suivante :

Théorème 0.2. Supposons que $\mathrm{Pl}_{p} \subset S \cup T, \mu_{p} \subset K$ et que $K_{\infty} / K$ contient la $\mathbb{Z}_{p}$-extension cyclotomique de $K$. Alors on a les identités

$$
\rho_{S}^{T}+\frac{\delta_{T}}{2}=\rho_{T}^{S}+\frac{\delta_{S}}{2}, \quad r_{S}^{T}=r_{T}^{S} \quad \text { et } \quad \mu_{S}^{T}=\mu_{T}^{S},
$$

avec $\delta_{S}=\sum_{v \in S \cap \mathrm{Pl}}\left[K_{v}: \mathbb{Q}_{p}\right]$.

On énonce également des formules de ce type, sous l'hypothèse moins restrictive que $K$ contient les racines $p$-ièmes de l'unité (théorèmes 3.8 pour $p=2$ et 3.9 pour $p$ impair). Elles rejoignent les formules de réflexion obtenues par Maire [2005] dans le cas commutatif. 
La partie 1 est dédié à la présentation des invariants d'Iwasawa d'un $\Lambda$-module de type fini. On rappelle les principales propriétés des groupes $p$-valués, établies par Lazard [1965], ainsi que de leur algèbre d'Iwasawa $\Lambda$. On introduit alors les invariant d'Iwasawa $\rho, \mu$ et $r$ d'un $\Lambda$-module de type fini, et on étudie leur comportement vis-à-vis des pseudo-isomorphismes.

La seconde partie, tout comme la première, est purement algébrique. Son but est la démonstration du théorème 2.1, qui indique qu'étant donné un $\Lambda$-module de type fini $M$, le comportement asymptotique de ses coïnvariants $M_{G_{n}}$ est contrôlé par ses invariants d'Iwasawa. Les démonstrations utilisent fortement la notion de pseudo-nullité dans le cadre non-commutatif, introduite par Venjakob [2002], ainsi que les théorèmes de structure à pseudo-isomorphisme près dont on dispose (voir aussi [Coates et al. 2003]).

Ces résultats sont ensuite appliqués à un cadre arithmétique. On démontre des formules "à la Iwasawa" concernant l'évolution asymptotique des $p$-groupes des classes généralisés le long d'une extension d'un corps de nombres de groupe de Galois uniforme. On en déduit des résultats de dualité entre les invariants d'Iwasawa, lorsque l'on intervertit les conditions de ramification et de décomposition. Ces résultats permettent, dans certains cas, d'effectuer des calculs d'invariants $\rho$.

\section{Sur les invariants d'Iwasawa}

Groupes p-valués. Les groupes $p$-adiques analytiques, qui sont les groupes topologiques possédant une structure compatible de variété analytique sur $\mathbb{Q}_{p}$, sont au coeur de la théorie d'Iwasawa non-commutative. On va se focaliser, dans cette étude, sur une certaine classe de groupes analytiques possédant de bonnes propriétés : les pro- $p$-groupes $p$-valués de type fini. L'introduction de ces groupes et leur étude remontent à Lazard [1965]. Les propriétés exposées dans cette section proviennent de ses travaux.

Définition 1.1. Un groupe $G$ est dit $p$-valué s'il est muni d'une application $\omega$ : $G \rightarrow \mathbb{R}_{+}^{*} \cup\{+\infty\}$, appelée filtration, vérifiant les axiomes :

(i) $\omega\left(x y^{-1}\right) \geqslant \min (\omega(x), \omega(y))$,

(ii) $\omega\left(x^{-1} y^{-1} x y\right) \geqslant \omega(x)+\omega(y)$,

(iii) $\omega(x)=+\infty \Longleftrightarrow x=1$,

(iv) $\omega(x)>(p-1)^{-1}$,

(v) $\omega\left(x^{p}\right)=\omega(x)+1$.

Proposition 1.2. Soit $G$ un groupe p-valué profini de type fini. Alors:

(1) Le groupe G est un pro-p-groupe analytique sans élément d'ordre fini.

(2) La filtration $\omega$ est discrète. 
On se donne dans la suite un pro-p-groupe $p$-valué de type fini $G$. On notera $d$ sa dimension (en tant que groupe analytique), que l'on suppose supérieure à 1 . On peut prendre un pro- $p$-groupe uniforme, pour la filtration induite par la suite centrale descendante (voir [Dixon et al. 1999]). Les exemples les plus classiques sont $\mathbb{Z}_{p}^{d}$ ou les sous-groupes de congruence des groupes linéaires $\mathrm{GL}_{r}\left(\mathbb{Z}_{p}\right)$.

On peut grossir la filtration initiale sur $G$ en posant, pour $n \in \mathbb{N}^{*}$ :

$$
G_{n}=\left\{x \in G \mid \omega(x)>(p-1)^{-1}+n-1\right\} .
$$

La filtration induite par les sous-groupes $G_{n}$ donne une structure de groupe $p$-valué sur $G$ dont les valeurs sont entières.

Proposition 1.3. Soit $G$ un pro-p-groupe p-valué de type fini de dimension d et $G_{n}$ les sous-groupes de $G$ définis ci-dessus. Il existe une constante $C$ telle que les indices des $G_{n}$ dans $G$ vérifient

$$
\left(G: G_{n}\right)=C p^{n d}
$$

pour $n$ assez grand.

Démonstration. On utilise les résultats et le vocabulaire de [Lazard 1965].

Pour $n_{0}$ assez grand, le groupe $G_{n_{0}}$ est $p$-saturé de dimension $d$ [ibid., III 3.1.13]. En particulier, $\left(G_{n_{0}}: G_{n_{0}+k}\right)=p^{k d}$ [ibid., III 3.1.8] et on a pour $n \geqslant n_{0}$

$$
\left(G: G_{n}\right)=\left(G: G_{n_{0}}\right)\left(G_{n_{0}}: G_{n}\right)=p^{c+\left(n-n_{0}\right) d} .
$$

Lorsque $G$ est uniforme, on a $G_{n}=G^{p^{n}}$ et la constante $C$ de la proposition 1.3 est égale à 1.

L'algèbre d'Iwasawa $\Lambda$. On énonce dans ce paragraphe quelques propriétés vérifiées par l'algèbre d'Iwasawa d'un pro- $p$-groupe $p$-valué de type fini $G$, de dimension $d$.

On définit l'algèbre d'Iwasawa associée à $G$ par

$$
\Lambda(G)=\Lambda:=\mathbb{Z}_{p} \llbracket G \rrbracket=\lim _{U \triangleleft_{o} G} \mathbb{Z}_{p}[G / U],
$$

où $U$ parcout l'ensemble des sous-groupes distingués ouverts de $G$.

On sera aussi amené à considérer l'algèbre complète de $G \operatorname{sur} \mathbb{F}_{p}$ :

$$
\Omega(G)=\Omega:=\mathbb{F}_{p} \llbracket G \rrbracket=\lim _{U \overleftarrow{\triangleleft_{o}} G} \mathbb{F}_{p}[G / U]
$$

Pour un sous-groupe $U$ de $G$, on définit l'idéal d'augmentation $I_{U}$ de $\Lambda$ (ou de $\Omega$ ) comme le noyau de la réduction modulo $U$.

Il est prouvé dans [Lazard 1965, II 2.2.2] que les algèbres $\Lambda$ et $\Omega$ sont locales, d'idéaux maximaux respectifs $p \Lambda+I_{G}$ et $I_{G}$. Cette même référence nous informe que les algèbres graduées $\operatorname{Gr}(\Lambda)=\bigoplus I_{G_{n}} / I_{G_{n+1}}$ et $\operatorname{Gr}(\Omega)$ sont des algèbres de 
polynômes à respectivement $d+1$ et $d$ variables [ibid., III 2.3.3]. En particulier, les algèbres $\Lambda$ et $\Omega$ sont noethériennes à droite et à gauche et intègres.

Invariants des $\Lambda$-modules. Maintenant, $\Lambda$ désignera l'algèbre d'Iwasawa d'un pro-p-groupe $p$-valué de type fini $G$. On s'intéresse alors aux $\Lambda$-modules de type fini, c'est-à-dire aux $\mathbb{Z}_{p}$-modules topologiques avec une action continue de $G$, qui sont de type fini pour leur structure de $\Lambda$-module étendant l'action de $G$. On va définir trois invariants pour de tels modules. Ils généralisent les invariants d'Iwasawa $\rho, \mu$ et $r$ qui apparaissent dans le théorème de structure lorsque le groupe $G$ est isomorphe à $\mathbb{Z}_{p}$, l'invariant $r$ désignant le nombre de facteurs dans la partie de $\mathbb{Z}_{p}$-torsion. Les invariants $\rho$ et $\mu$ ont déjà fait l'objet d'études de la part de Harris [1979], Venjakob [2002] et Howson [2002].

Définition 1.4. Soit $M$ un $\Lambda$-module de type fini. Pour $i \in \mathbb{N}$ on note par $M\left[p^{i}\right]$ les éléments de $M$ tués par $p^{i}$. On définit les invariants d'Iwasawa de $M$ par

$$
\begin{aligned}
\rho(M) & =\operatorname{rk}_{\Lambda}(M)=\operatorname{dim}_{F}\left(F \otimes_{\Lambda} M\right), \\
r(M) & =\operatorname{rk}_{\Omega}(M[p]), \\
\mu(M) & =\sum_{i \geq 0} \operatorname{rk}_{\Omega}\left(M\left[p^{i+1}\right] / M\left[p^{i}\right]\right),
\end{aligned}
$$

avec $F$ le corps gauche des fractions de $\Lambda$ (voir [Lesieur et Croisot 1959]).

L'invariant $\rho$ est le rang de $M$. Il est additif sur les suites exactes de $\Lambda$-modules et un $\Lambda$-module $M$ est de torsion si et seulement si $\rho(M)=0$. Quant aux invariants $r$ et $\mu$, ils mesurent la $\mathbb{Z}_{p}$-torsion de $M$. L'invariant $\mu$ ne dépend que du sous-module de $\mathbb{Z}_{p}$-torsion de $M$ et est additif sur les suites exactes de modules de $\Lambda$-torsion (voir [Venjakob 2002, corollaire 3.37]), tandis que $r$ ne dépend que de $M$ [ $p$ ] et est additif sur les suites exactes de modules tués par $p$. Ces deux derniers invariants sont aussi donnés par les formules suivantes :

Lemme 1.5. Pour $M$ un $\Lambda$-module de type fini, on a

$$
\begin{aligned}
r(M) & =\operatorname{rk}_{\Omega}\left(\operatorname{tor}_{\mathbb{Z}_{p}}(M) / p\right)=\operatorname{rk}_{\Omega}\left(\operatorname{tor}_{\Lambda}(M) / p\right), \\
\mu(M) & =\sum_{i \geq 0} \operatorname{rk}_{\Omega}\left(p^{i} \operatorname{tor}_{\mathbb{Z}_{p}}(M) / p^{i+1} \operatorname{tor}_{\mathbb{Z}_{p}}(M)\right) \\
& =\sum_{i \geq 0} \operatorname{rk}_{\Omega}\left(p^{i} \operatorname{tor}_{\Lambda}(M) / p^{i+1} \operatorname{tor}_{\Lambda}(M)\right) .
\end{aligned}
$$

Démonstration. Notons dans cette preuve $T=\operatorname{tor}_{\mathbb{Z}_{p}}(M)$. L'anneau $\Lambda$ étant noethérien, il existe un entier $\alpha$ tel que $T=M\left[p^{\alpha}\right]$.

Quotienter par $p$ la suite exacte

$$
0 \rightarrow p T \rightarrow T \rightarrow T / p \rightarrow 0
$$


conduit à la suite exacte de $\Omega$-modules

$$
0 \rightarrow(p T)[p] \rightarrow T[p] \rightarrow T / p \rightarrow(p T) / p \rightarrow T / p \rightarrow T / p \rightarrow 0 .
$$

La somme alternée des $\Omega$-rangs nous informe que

$$
\operatorname{rk}_{\Omega}(T[p])-\operatorname{rk}_{\Omega}(T / p)=\operatorname{rk}_{\Omega}((p T)[p])-\mathrm{rk}_{\Omega}((p T) / p) .
$$

Il est possible d'itérer les calculs en remplaçant $T$ par $p T$. On obtient, pour tout entier $i$,

$$
\mathrm{rk}_{\Omega}(T[p])-\mathrm{rk}_{\Omega}(T / p)=\operatorname{rk}_{\Omega}\left(\left(p^{i} T\right)[p]\right)-\mathrm{rk}_{\Omega}\left(\left(p^{i} T\right) / p\right)=0,
$$

la nullité provenant du cas $i=\alpha$. On justifie la première égalité du lemme pour $r$ et $\mu$ en notant que

$$
T\left[p^{i+1}\right] / T\left[p^{i}\right] \simeq p^{i}\left(T\left[p^{i+1}\right]\right)=\left(p^{i} T\right)[p] .
$$

Enfin, pour voir que l'on peut calculer $\mu$ et $r$ à partir du sous-module tor ${ }_{\Lambda}(M)$, on regarde pour tout $i \in \mathbb{N}$ la suite exacte

$$
0 \rightarrow p^{i} T \rightarrow p^{i} \operatorname{tor}_{\Lambda}(M) \rightarrow p^{i} \operatorname{tor}_{\Lambda}(M) / p^{i} T \rightarrow 0 .
$$

Le dernier terme de cette suite, que l'on note $N$, est un $\Lambda$-module de torsion sans $p$-torsion. Quotienter par $p$ donne donc

$$
0 \rightarrow p^{i} T / p^{i+1} T \rightarrow p^{i} \operatorname{tor}_{\Lambda}(M) / p^{i+1} \operatorname{tor}_{\Lambda}(M) \rightarrow N / p \rightarrow 0,
$$

où $N / p$ est un $\Omega$-module de torsion. L'additivité du rang permet de conclure que

$$
\operatorname{rk}_{\Omega}\left(p^{i} T / p^{i+1} T\right)=\operatorname{rk}_{\Omega}\left(p^{i} \operatorname{tor}_{\Lambda}(M) / p^{i+1} \operatorname{tor}_{\Lambda}(M)\right) .
$$

Pseudo-nullité et invariants. Beaucoup de résultats de la théorie d'Iwasawa classique, lorsque $G \simeq \mathbb{Z}_{p}$, reposent sur le théorème de structure des $\Lambda$-modules de type fini à pseudo-isomorphisme près (voir par exemple [Serre 1966]). Coates et al. [2003], ainsi que Venjakob [2002], ont ouvert la voie vers une généralisation au cas non-commutatif de ce théorème. On rappelle la notion de pseudo-nullité qu'ils ont introduite dans ce contexte. La notation $\operatorname{Ext}_{\Lambda}(-,-)$ désignera les dérivés du bifoncteur $\operatorname{Hom}_{\Lambda}(-,-)$ des homomorphismes continus entre $\Lambda$-modules de type fini.

Définition 1.6. Un $\Lambda$-module de type fini $M$ est dit pseudo-nul si

$$
\operatorname{Ext}_{\Lambda}^{0}(M, \Lambda)=\operatorname{Ext}_{\Lambda}^{1}(M, \Lambda)=0 .
$$

Un morphisme $f: M \rightarrow N$ entre deux $\Lambda$-modules de type fini est appelé pseudoisomorphisme si le noyau et le conoyau de $f$ sont pseudo-nuls. 
Le lemme suivant indique que la sous-catégorie des modules pseudo-nuls vérifie la condition de Serre et permet de considérer la catégorie quotient.

Lemme 1.7. Tout sous-module et tout quotient d'un module pseudo-nul est pseudonul.

Démonstration. C'est la proposition 3.6 de [Venjakob 2002].

On démontre alors la proposition suivante, qui met en évidence le fait que les invariants d'Iwasawa se comportent bien vis-à-vis des pseudo-isomorphismes.

Proposition 1.8. Soit $M$ et $N$ deux $\Lambda$-modules de type fini pseudo-isomorphes. Alors $\rho(M)=\rho(N), \mu(M)=\mu(N)$ et $r(M)=r(N)$.

Démonstration. La condition $\operatorname{Ext}_{\Lambda}^{0}(A, \Lambda)=\operatorname{Hom}_{\Lambda}(A, \Lambda)=0$ indique que tout $\Lambda$ module pseudo-nul $A$ est de torsion. Ceci entraîne que $\rho(A)=0$ et, comme $\rho$ est aussi additif sur les suites exactes, il est invariant modulo pseudo-isomorphisme.

En ce qui concerne $r$, on écrit la suite exacte donnée par le pseudo-isomorphisme

$$
0 \rightarrow A \rightarrow M \stackrel{f}{\rightarrow} N \rightarrow B \rightarrow 0
$$

et on note $Z=\operatorname{Im}(f)$. Le lemme du serpent permet d'extraire les deux suites exactes

$$
A[p] \rightarrow M[p] \rightarrow Z[p] \rightarrow A / p \quad \text { et } \quad 0 \rightarrow Z[p] \rightarrow N[p] \rightarrow B[p] .
$$

D'après le lemme 1.7, les termes extrémaux de ces deux suites sont des modules pseudo-nuls tués par $p$. Le lemme 1.9 ci-dessous donne alors $r(M)=r(Z)=r(N)$ en regardant les $\Omega$-rangs des termes des deux suites.

Lemme 1.9. Si A est un $\Lambda$-module pseudo-nul tué par p, alors A est un $\Omega$-module de torsion.

Démonstration. Soit $A$ un $\Lambda$-module pseudo-nul tué par $p$. La suite exacte

$$
0 \rightarrow \Lambda \stackrel{p}{\rightarrow} \Lambda \rightarrow \Omega \rightarrow 0
$$

conduit à l'encadrement

$$
\operatorname{Ext}_{\Lambda}^{0}(A, \Lambda) \rightarrow \operatorname{Hom}_{\Lambda}(A, \Omega) \rightarrow \operatorname{Ext}_{\Lambda}^{1}(A, \Lambda) .
$$

La pseudo-nullité de $A$ entraîne la nullité de $\operatorname{Hom}_{\Lambda}(A, \Omega)$, qui coïncide avec $\operatorname{Hom}_{\Omega}(A, \Omega)$. Le $\Omega$-module $A$ est donc de torsion.

En ce qui concerne $\mu$, on utilise des arguments similaires exposés dans la preuve de la proposition 3.34 de [Venjakob 2002]. 
On conclut ce paragraphe par un théorème qui donne la structure, à pseudoisomorphisme près, de la $\mathbb{Z}_{p}$-torsion d'un $\Lambda$-module de type fini. Il permet, comme dans le cadre de la théorie commutative, de lire les invariants $\mu$ et $r$ sur un module élémentaire.

Théorème 1.10 [Venjakob 2002, théorème 3.40]. Soit $M$ un $\Lambda$-module de type fini. Il existe des uniques entiers naturels $\alpha_{1}, \ldots, \alpha_{r}$ tels que l'on ait un pseudoisomorphisme

$$
\operatorname{tor}_{\mathbb{Z}_{p}}(M) \rightarrow \bigoplus_{i=1}^{r} \Lambda / p^{\alpha_{i}} .
$$

De plus, $r(M)=r$ et $\mu(M)=\sum_{i=1}^{r} \alpha_{i}$.

\section{Comportement asymptotique des coïnvariants}

On se donne $G$ un pro-p-groupe $p$-valué de type fini de dimension $d \geqslant 1$. On le munit de sa filtration entière $\left(G_{n}\right)_{n}$ (voir page 823 ), et on pose $\Lambda=\mathbb{Z}_{p} \llbracket G \rrbracket$. Pour $M$ un $\Lambda$-module de type fini, on notera $M_{G_{n}}$ le plus grand quotient du module $M$ sur lequel $G_{n}$ agit trivialement. C'est le module des coïnvariants de $M$, qui s'obtient comme quotient

$$
M_{G_{n}}=M / I_{G_{n}} M \simeq M \hat{\otimes}_{\Lambda\left(G_{n}\right)} \mathbb{Z}_{p},
$$

où $\hat{\otimes}_{\Lambda\left(G_{n}\right)}$ désigne le produit tensoriel complété (voir [Brumer 1966, section 2]).

Si $G$ est isomorphe à $\mathbb{Z}_{p}$, le contenu de [Jaulent 2005], permet de voir que les invariants d'Iwasawa de $M$ sont reliés aux cardinaux de certains quotients finis de ses coïnvariants. C'est ce type de résultats que l'on voudrait étendre à des extensions de groupe de Galois $p$-valué. Plus précisément, on démontre les deux résultats suivants :

Théorème 2.1. Soit $M$ un $\Lambda$-module de type fini d'invariants $\rho, \mu$ et $r$. Alors :

(i) $\operatorname{dim}_{\mathbb{F}_{p}}\left(M_{G_{n}} / p\right)=(\rho+r)\left(G: G_{n}\right)+O\left(p^{n(d-1)}\right)$.

(ii) $\#\left(M_{G_{n}} / p^{n}\right)=p^{(\rho n+\mu)\left(G: G_{n}\right)+O\left(n p^{n(d-1)}\right)}$.

Rappelons que $\left(G: G_{n}\right)=C p^{n d}$ pour $n$ assez grand, avec $C=1$ si $G$ est uniforme.

Un théorème de Harris. Le point de départ de la preuve du théorème 2.1 est un résultat de Harris [2000, théorème 1.10], que l'on formule de la façon suivante :

Théorème 2.2. Si $M$ est un $\Omega$-module de type fini, alors

$$
\operatorname{dim}_{\mathbb{F}_{p}}\left(M_{G_{n}}\right)=\operatorname{rk}_{\Omega}(M)\left(G: G_{n}\right)+O\left(p^{n(d-1)}\right) .
$$


Démonstration. Pour $M$ de $\Omega$-torsion, les arguments de la preuve du théorème 1.10 de [Harris 2000] s'adaptent à un groupe $p$-valué et permettent de voir que $\operatorname{dim}_{\mathbb{F}_{p}}\left(M_{G_{n}}\right)=O\left(p^{n(d-1)}\right)$.

Dans le cas général, on dévisse pour se ramener d'abord à un $\Omega$-module sans torsion, puis à un $\Omega$-module libre.

Pour $i$ et $n$ des entiers naturels, les groupes d'homologie de $G_{n}$ à coefficients dans un $\Lambda$-module de type fini $M$ sont définis par

$$
H_{i}\left(G_{n}, M\right)=\operatorname{Tor}_{i}^{\Lambda\left(G_{n}\right)}\left(\mathbb{Z}_{p}, M\right),
$$

où $\operatorname{Tor}_{i}^{\Lambda\left(G_{n}\right)}(-,-)$ désignent les dérivés du bifoncteur $-\hat{\otimes}_{\Lambda\left(G_{n}\right)}-$.

Lorsque $M$ est aussi muni d'une structure de $\Omega$-module, le théorème 2.2 donne des renseignements sur $H_{0}\left(G_{n}, M\right)=M_{G_{n}}$. En effet, on a dans ce cas

$$
H_{i}\left(G_{n}, M\right)=\operatorname{Tor}_{i}^{\Lambda\left(G_{n}\right)}\left(\mathbb{Z}_{p}, M\right) \simeq \operatorname{Tor}_{i}^{\Omega\left(G_{n}\right)}\left(\mathbb{F}_{p}, M\right)
$$

(voir [Ribes et Zalesskii 2000, lemme 6.3.5]).

Le corollaire suivant étend le théorème 2.2 aux groupes d'homologie supérieurs pour les $\Omega$-modules de torsion.

Corollaire 2.3. Soit $M$ un $\Omega$-module de type fini et de torsion. Pour tout $i \geqslant 0$, on $a$

$$
\operatorname{dim}_{\mathbb{F}_{p}}\left(H_{i}\left(G_{n}, M\right)\right)=O\left(p^{n(d-1)}\right) .
$$

Démonstration. On procède par récurrence sur $i$, le cas $i=0$ étant donné par le théorème 2.2.

Le $\Omega$-module $M$ étant de type fini et de torsion, il existe un $\Omega$-module de torsion $A$ et des éléments non nuls $f_{1}, \ldots, f_{r} \in \Omega$ prenant place dans la suite exacte

$$
0 \rightarrow A \rightarrow \bigoplus_{j} \Omega / f_{j} \rightarrow M \rightarrow 0 .
$$

Cette suite conduit à l'encadrement

$$
H_{i}\left(G_{n}, \bigoplus_{j} \Omega / f_{j}\right) \rightarrow H_{i}\left(G_{n}, M\right) \rightarrow H_{i-1}\left(G_{n}, A\right) .
$$

L'hypothèse de récurrence appliquée à $A$ entraîne

$$
\operatorname{dim}_{\mathbb{F}_{p}}\left(H_{i-1}\left(G_{n}, A\right)\right)=O\left(p^{n(d-1)}\right)
$$

et permet de se ramener au cas $M=\Omega / f$.

On calcule les groupes d'homologie de $\Omega / f$ à partir de sa résolution $\Omega\left(G_{n}\right)$ libre

$$
0 \rightarrow \Omega \stackrel{f}{\rightarrow} \Omega \rightarrow \Omega / f \rightarrow 0
$$


On obtient immédiatement $H_{i}\left(G_{n}, \Omega / f\right)=0$ pour $i>1$, ainsi que les renseignements suivants sur le $H_{1}$ :

$$
\begin{aligned}
\operatorname{dim}_{\mathbb{F}_{p}}\left(H_{1}\left(G_{n}, \Omega / f\right)\right) & =\operatorname{dim}_{\mathbb{F}_{p}}\left(\operatorname{ker}\left(\Omega_{G_{n}} \stackrel{f}{\rightarrow} \Omega_{G_{n}}\right)\right) \\
& =\operatorname{dim}_{\mathbb{F}_{p}}\left(\operatorname{coker}\left(\Omega_{G_{n}} \stackrel{f}{\rightarrow} \Omega_{G_{n}}\right)\right) \\
& =\operatorname{dim}_{\mathbb{F}_{p}}\left((\Omega / f)_{G_{n}}\right)=O\left(p^{n(d-1)}\right),
\end{aligned}
$$

la dernière égalité provenant du théorème 2.2.

On termine ce paragraphe par une dernière variante du théorème de Harris.

Corollaire 2.4. Soit $M$ un $\Lambda$-module de type fini tel que $M / p$ est un $\Omega$-module de torsion. Alors, pour tout $i \geqslant 0$,

$$
\#\left(H_{i}\left(G_{n}, M / p^{n}\right)\right)=p^{O\left(n p^{n(d-1)}\right)} .
$$

Démonstration. On va découper $M / p^{n} M$ selon les $p^{k} M / p^{k+1} M$. Le corollaire 2.3 fournit des constantes $C_{k}$, indépendantes de $n$, telles que

$$
\# H_{i}\left(G_{n}, p^{k} M / p^{k+1} M\right) \leqslant p^{C_{k} p^{n(d-1)}} .
$$

Pour $k<n$, la $G_{n}$-homologie de la suite exacte

$$
0 \rightarrow p^{k+1} M / p^{n} M \rightarrow p^{k} M / p^{n} M \rightarrow p^{k} M / p^{k+1} M \rightarrow 0
$$

entraîne les inégalités

$$
\# H_{i}\left(G_{n}, p^{k} M / p^{n} M\right) \leqslant \# H_{i}\left(G_{n}, p^{k+1} M / p^{n} M\right) p^{C_{k} p^{n(d-1)}} .
$$

Elles conduisent finalement à

$$
\# H_{i}\left(G_{n}, M / p^{n} M\right) \leqslant p^{\left(C_{1}+\cdots+C_{n}\right) p^{n(d-1)}} .
$$

Pour $k$ assez grand, le module $p^{k} M$ est sans $p$-torsion. Ainsi

$$
p^{k} M / p^{k+1} M \simeq p^{k+1} M / p^{k+2} M
$$

et les constantes $C_{k}$ sont majorées par une constante $C^{\prime}$ indépendante de $k$. On en tire

$$
\# H_{i}\left(G_{n}, M / p^{n} M\right) \leqslant p^{C^{\prime} n p^{n(d-1)}} .
$$


Preuve du théorème $2.1 \mathrm{i}$ ). Pour un $\Lambda$-module $M$ de type fini, on cherche à dé-

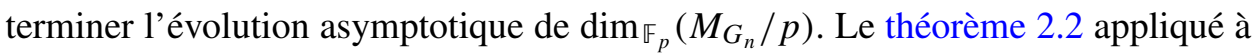
$M / p$ donne l'estimation

$$
\operatorname{dim}_{\mathbb{F}_{p}}\left(M_{G_{n}} / p\right)=\operatorname{rk}_{\Omega}(M / p)\left(G: G_{n}\right)+O\left(p^{n(d-1)}\right) .
$$

Le corollaire 1.10 de [Howson 2002] relie la valeur de $\operatorname{rk}_{\Omega}(M / p)$ aux invariants d'Iwasawa de $M$ de la façon suivante :

$$
\operatorname{rk}_{\Omega}(M / p)=\operatorname{rk}_{\Omega}(M[p])+\operatorname{rk}_{\Lambda}(M)=r+\rho .
$$

La combinaison de ces deux résultats donne la première formule du théorème 2.1 .

Preuve du théorème 2.1 ii). Soit $M$ un $\Lambda$-module de type fini. Pour démontrer le deuxième point du théorème 2.1 , on va utiliser des résultats de structure modulo pseudo-isomorphisme pour $M$. Notons que les techniques de cette section fournissent également une preuve du point i) du théorème.

On commence par remarquer que la quantité $\#\left(M_{G_{n}} / p^{n}\right)$ que l'on veut calculer passe bien modulo pseudo-isomorphisme :

Proposition 2.5. Soient $M$ et $N$ deux $\Lambda$-modules de type fini pseudo-isomorphes. Alors

$$
\#\left(M_{G_{n}} / p^{n}\right)=\#\left(N_{G_{n}} / p^{n}\right) p^{O\left(n p^{n(d-1)}\right)} .
$$

Démonstration. Soit

$$
0 \rightarrow A \rightarrow M \stackrel{f}{\rightarrow} N \rightarrow B \rightarrow 0
$$

la suite exacte donnée par le pseudo-isomorphisme et soit $Z=\operatorname{Im}(f)$. On en déduit par passage au quotient les suites exactes

$$
A_{G_{n}} / p^{n} \rightarrow M_{G_{n}} / p^{n} \rightarrow Z_{G_{n}} / p^{n} \rightarrow 0
$$

et

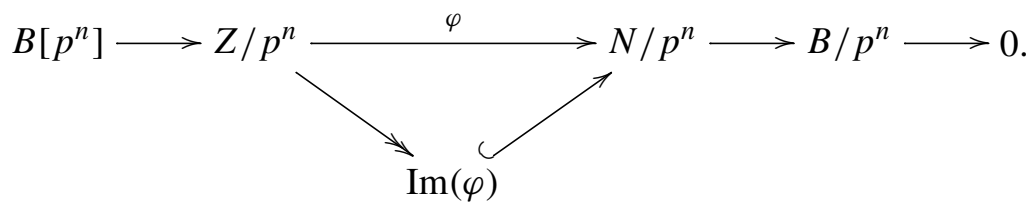

Cette dernière se découpe pour donner

$$
B\left[p^{n}\right]_{G_{n}} \rightarrow Z_{G_{n}} / p^{n} \rightarrow \operatorname{Im}(\varphi)_{G_{n}} \rightarrow 0
$$

et

$$
H_{1}\left(G_{n}, B / p^{n}\right) \rightarrow \operatorname{Im}(\varphi)_{G_{n}} \rightarrow N_{G_{n}} / p^{n} \rightarrow B_{G_{n}} / p^{n} \rightarrow 0 .
$$


Le lemme 1.9 permet d'appliquer le corollaire 2.4 à tout module pseudo-nul. Ceci fournit successivement :

$$
\begin{aligned}
\#\left(M_{G_{n}} / p^{n}\right) & =\#\left(Z_{G_{n}} / p^{n}\right) p^{O\left(n p^{n(d-1)}\right)} \\
& =\#(\operatorname{Im}(\varphi)) p^{O\left(n p^{n(d-1)}\right)}=\#\left(N_{G_{n}} / p^{n}\right) p^{O\left(n p^{n(d-1)}\right)} .
\end{aligned}
$$

La première étape consite à séparer l'étude des $\Lambda$-modules de torsion de celle des $\Lambda$-modules sans torsion. On considère la catégorie quotient des $\Lambda$-modules de type fini par la sous-catégorie des modules pseudo-nuls (lemme 1.7) et on note $Q$ le foncteur canonique de passage au quotient. On dispose du résultat de structure suivant :

Proposition 2.6 [Coates et al. 2003, proposition 6.4]. Soit M un $\Lambda$-module de type fini. On a un isomorphisme dans la catégorie quotient :

$$
Q(M) \simeq Q\left(\operatorname{tor}_{\Lambda}(M)\right) \oplus Q(\tilde{M}),
$$

où $\widetilde{M}$ désigne le quotient de M par son sous-module de $\Lambda$-torsion.

Remarquons que $\operatorname{tor}_{\Lambda}(M)$ est un $\Lambda$-module de torsion dont les invariants $\mu$ et $r$ sont égaux à ceux de $M$, tandis que $\widetilde{M}$ est un $\Lambda$-module sans torsion de même rang que $M$. Le corollaire suivant permet d'étudier $\operatorname{tor}_{\Lambda}(M)$ d'une part et $\widetilde{M}$ d'autre part.

Corollaire 2.7. Avec les notations de la proposition précédente, on a:

$$
\#\left(M_{G_{n}} / p^{n}\right)=\#\left(\operatorname{tor}_{\Lambda}(M)_{G_{n}} / p^{n}\right) \#\left(\tilde{M}_{G_{n}} / p^{n}\right) p^{O\left(n p^{n(d-1)}\right)} .
$$

Démonstration. L'isomorphisme dans la catégorie quotient qui apparaît dans la proposition 2.6 signifie qu'il existe des modules pseudo-nuls $A, B, C$ et un sousmodule $M^{\prime}$ de $M$, avec $M / M^{\prime}$ pseudo-nul, prenant place dans le diagramme suivant (voir [Gabriel 1962, chapitre III]) :

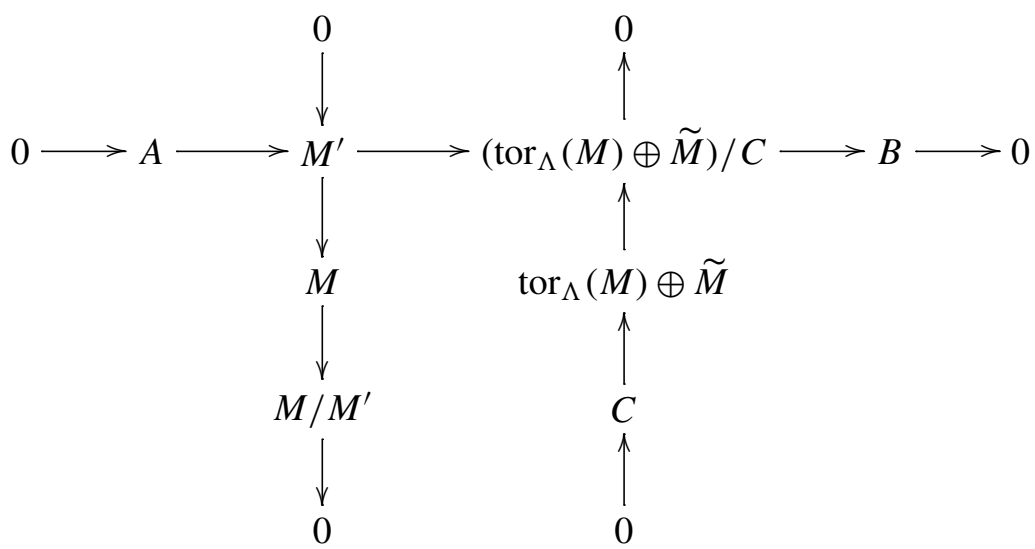


On a donc des pseudo-isomorphismes entre $M^{\prime}$ et $M$, entre $M^{\prime}$ et $\left(\operatorname{tor}_{\Lambda}(M) \oplus \tilde{M}\right) / C$ et entre $\operatorname{tor}_{\Lambda}(M) \oplus \widetilde{M}$ et $\left(\operatorname{tor}_{\Lambda}(M) \oplus \widetilde{M}\right) / C$. On applique alors la proposition 2.5 pour obtenir :

$$
\begin{aligned}
\#\left(M_{G_{n}} / p^{n}\right) & =\#\left(M_{G_{n}}^{\prime} / p^{n}\right) p^{O\left(n p^{n(d-1)}\right)} \\
& =\#\left(\left(\operatorname{tor}_{\Lambda}(M) \oplus \widetilde{M} / C\right)_{G_{n}} / p^{n}\right) p^{O\left(n p^{n(d-1)}\right)} \\
& =\#\left(\left(\operatorname{tor}_{\Lambda}(M) \oplus \widetilde{M}\right)_{G_{n}} / p^{n}\right) p^{O\left(n p^{n(d-1)}\right)} \\
& =\#\left(\operatorname{tor}_{\Lambda}(M)_{G_{n}} / p^{n}\right) \#\left(\tilde{M}_{G_{n}} / p^{n}\right) p^{O\left(n p^{n(d-1)}\right)} .
\end{aligned}
$$

On détermine, dans le lemme suivant, la quantité \#( $\left.\operatorname{tor}_{\Lambda}(M)_{G_{n}} / p^{n}\right)$.

Lemme 2.8. Soit $T$ un $\Lambda$-module de type fini, de torsion et d'invariant $\mu$. Alors

$$
\#\left(T_{G_{n}} / p^{n}\right)=p^{\mu\left(G: G_{n}\right)+O\left(n p^{n(d-1)}\right)} .
$$

Démonstration. On dévisse le problème en s'intéressant à la suite exacte

$$
0 \rightarrow \operatorname{tor}_{\mathbb{Z}_{p}}(T) \rightarrow T \rightarrow \bar{T} \rightarrow 0,
$$

où $\bar{T}=T /$ tor $_{\mathbb{Z}_{p}}(M)$ est un module de $\Lambda$-torsion sans $\mathbb{Z}_{p}$-torsion. Le module $\bar{T}$ étant sans $\mathbb{Z}_{p}$-torsion, quotienter par $p^{n}$ conserve l'exactitude de cette suite et conduit à la suite exacte d'homologie

$$
H_{1}\left(G_{n}, \bar{T} / p^{n}\right) \rightarrow \operatorname{tor}_{\mathbb{Z}_{p}}(T)_{G_{n}} / p^{n} \rightarrow T_{G_{n}} / p^{n} \rightarrow \bar{T}_{G_{n}} / p^{n} \rightarrow 0 .
$$

Le corollaire 2.4, appliqué au module $\bar{T}$, permet d'obtenir la relation

$$
\#\left(T_{G_{n}} / p^{n}\right)=\#\left(\operatorname{tor}_{\mathbb{Z}_{p}}(T)_{G_{n}} / p^{n}\right) p^{O\left(n p^{n(d-1)}\right)} .
$$

Il suffit donc de contôler le cardinal de $\operatorname{tor}_{\mathbb{Z}_{p}}(T)_{G_{n}} / p^{n}$ à l'aide du théorème 1.10, qui fournit un pseudo-isomorphisme

$$
\operatorname{tor}_{\mathbb{Z}_{p}}(T) \rightarrow \bigoplus_{i=1}^{r} \Lambda / p^{\alpha_{i}},
$$

avec $\mu=\sum \alpha_{i}$. La proposition 2.5 nous informe alors que

$$
\begin{aligned}
\#\left(\operatorname{tor}_{\mathbb{Z}_{p}}(T)_{G_{n}} / p^{n}\right) & =\#\left(\bigoplus_{i=1}^{r} \mathbb{Z}_{p}\left[G / G_{n}\right] /\left(p^{\alpha_{i}}, p^{n}\right)\right) p^{O\left(n p^{n(d-1)}\right)} \\
& =p^{\mu\left(G: G_{n}\right)+O\left(n p^{n(d-1)}\right)} .
\end{aligned}
$$

Il reste à évaluer la quantité \# $\left(\widetilde{M}_{G_{n}} / p^{n}\right)$. C'est l'objet du lemme :

Lemme 2.9. Soit $M$ un $\Lambda$-module de type fini, sans-torsion et de rang $\rho$. Alors

$$
\#\left(M_{G_{n}} / p^{n}\right)=p^{\rho n\left(G: G_{n}\right)+O\left(n p^{n(d-1)}\right)} .
$$


Démonstration. Tout $\Lambda$-module sans torsion est contenu dans un module libre de même rang. On a donc une suite exacte

$$
0 \rightarrow M \rightarrow \Lambda^{\rho} \rightarrow T \rightarrow 0
$$

qui conduit à la suite exacte de $\Omega$-modules

$$
0 \rightarrow T[p] \rightarrow M / p \rightarrow \Omega^{\rho} \rightarrow T / p \rightarrow 0 .
$$

La somme alternée des $\Omega$-rangs nous informe que $r(M / p)=\rho$.

Le théorème $2.1 \mathrm{i}$ ) appliqué au $\Lambda$-module de torsion $M / p$ donne alors une suite bornée $\left(c_{n}\right)_{n}$ telle que

$$
\operatorname{dim}_{\mathbb{F}_{p}}\left(M_{G_{n}} / p\right)=\rho\left(G: G_{n}\right)+c_{n} p^{n(d-1)} .
$$

Pour calculer $\#\left(M_{G_{n}} / p^{n}\right)$, on remarque que l'on a les isomorphismes suivants, du fait que $M$ est sans torsion :

$$
p^{i}: M / p M \stackrel{\sim}{\longrightarrow} p^{i} M / p^{i+1} M .
$$

On a ainsi

$$
\begin{aligned}
\#\left(M_{G_{n}} / p^{n}\right) & =\prod_{i=0}^{n} \#\left(p^{i} M / p^{i+1} M\right)_{G_{n}} \\
& =\left(\#\left(M_{G_{n}} / p\right)\right)^{n} \\
& =p^{\rho n\left(G: G_{n}\right)+c_{n} n p^{n(d-1)}} \\
& =p^{\rho n\left(G: G_{n}\right)+O\left(n p^{n(d-1)}\right)} .
\end{aligned}
$$

La combinaison du corollaire 2.7 et des lemmes 2.8 et 2.9 fournit une preuve du théorème 2.1 ii).

\section{Applications arithmétiques}

On va appliquer les résultats de la partie 2 à une situation arithmétique faisant intervenir naturellement des $\Lambda$-modules. Plus précisément, on va étudier les invariants associés aux groupes de Galois de pro- $p$-extensions abéliennes maximales avec des conditions de ramification restreinte et de décomposition.

Le contexte arithmétique. On se fixe un corps de nombres $K$, qui fera office de corps de base. Soit $K_{\infty} / K$ une extension galoisienne vérifiant les hypothèses suivantes :

- Le groupe $G=\operatorname{Gal}\left(K_{\infty} / K\right)$ est un pro- $p$-groupe $p$-valué de type fini de dimension $d \geqslant 1$.

- Il n'y a qu'un nombre fini de places de $K$ ramifiées dans $K_{\infty} / K$. 
L'exemple historique est celui des $\mathbb{Z}_{p}$-extensions et $\mathbb{Z}_{p}$-extensions multiples, qui sont abéliennes. Un exemple avec $G$ non-commutatif est la fausse courbe de Tate, donnée par l'extension $\mathbb{Q}\left(\zeta_{p}, \sqrt[p]{p}\right) / \mathbb{Q}\left(\zeta_{p}\right)$. Son groupe de Galois est isomorphe à un produit semi-direct $\mathbb{Z}_{p} \rtimes \mathbb{Z}_{p}$. Dans ces deux premières illustrations, seules les places au-dessus de $p$ sont ramifiées.

On obtient aussi de telles extensions en regardant l'action du groupe de Galois absolu $G_{K}=\operatorname{Gal}(\bar{K} / K)$ sur les objets géométriques $H_{e t}^{i}\left(\bar{Y}, \mathbb{Q}_{p}\right)(j)$, où $Y$ est une variété projective lisse absolument irréductible définie sur $K$. Lorsque $Y$ est une courbe elliptique non $\mathrm{CM}$ fixée, on réalise ainsi des $\mathrm{GL}_{2}\left(\mathbb{Z}_{p}\right)$-extension, à part pour un nombre fini de premiers $p$. Ces extensions sont non-ramifiées en dehors de $p$ et des places de mauvaise réduction de $Y$ (voir [Serre 1972]).

C'est dans ce contexte que l'on fait intervenir les $\Lambda$-modules d'Iwasawa. Soient $S$ et $T$ deux ensembles finis disjoints de places de $K$. On écrira encore, si aucune confusion n'est possible, $S$ et $T$ pour les places d'une extension $L$ de $K$ qui sont au-dessus de celles de $S$ et $T$.

Soit $H_{S}^{T}$ la pro- $p$-extension abélienne $S$-ramifiée et $T$-décomposée maximale de $K_{\infty}$. On note $X_{S, \infty}^{T}=\operatorname{Gal}\left(H_{S}^{T} / K_{\infty}\right)$. On rappelle que $S$-ramifiée et $T$-décomposée signifie que seules les places de $S$ peuvent se ramifier dans cette extension et que les places de $T$ sont totalement décomposées. Pour une place infinie, "ramification" signifiera "complexification", tandis que "décomposition" sera synonyme de "noncomplexification". Par maximalité de $H_{S}^{T} / K_{\infty}$, l'extension $H_{S}^{T} / K$ est galoisienne et on obtient une action de $G \operatorname{sur} X_{S, \infty}^{T}$ par conjugaison, qui induit une structure de $\Lambda$-module sur $X_{S, \infty}^{T}$.

On munit $G$ de sa filtation entière par les sous-groupes $G_{n}$ (voir page 822) et note $K_{n}$ le sous-corps de $K_{\infty}$ fixé par $G_{n}$. On introduit aussi $\varphi_{=}=\operatorname{Gal}\left(H_{S}^{T} / K\right)$ et $\varphi_{n}=\operatorname{Gal}\left(H_{S}^{T} / K_{n}\right)$. Les notations prennent place dans le treillis suivant :

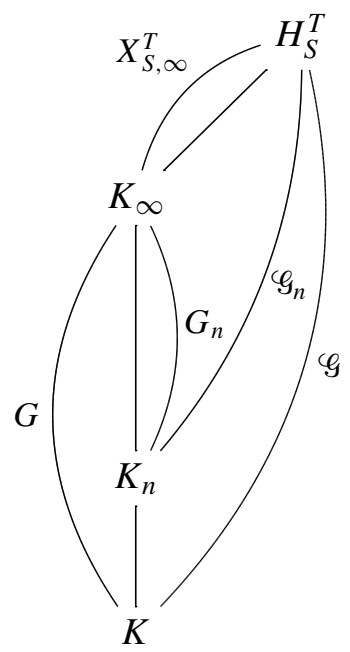


Un autre moyen de récupérer le module $X_{S, \infty}^{T}$ est de le construire par limite projective. Pour tout $n$, on va désigner $\operatorname{par} X_{S, n}^{T}$ le groupe de Galois de la pro$p$-extension abélienne $S$-ramifiée et $T$-décomposée maximale de $K_{n}$. Le groupe $X_{S, \infty}^{T}$ se retrouve alors comme la limite projective des $X_{S, n}^{T}$ pour les applications de restriction. Chaque $X_{S, n}^{T}$ étant muni d'une structure de $\mathbb{Z}_{p}\left[G / G_{n}\right]$-module, compatible avec les applications de restriction, la structure de $\Lambda$-module de $X_{S, \infty}^{T}$ est héritée de cette construction.

La proposition suivante autorise à introduire les invariants $\rho_{S}^{T}, \mu_{S}^{T}$ et $r_{S}^{T}$ du module d'Iwasawa $X_{S, \infty}^{T}$ (voir définition 1.4). On parlera aussi d'invariants de l'extension $K_{\infty} / K$.

Proposition 3.1. Le $\Lambda$-module $X_{S, \infty}^{T}$ est de type fini.

Démonstration. Le résultat peut se voir sur le diagramme du lemme 4.3 de [Jannsen 1989] mais on en donne une preuve plus directe ici. On oublie les indices $S$ et $T$ dans cette preuve.

On obtient la suite exacte

$$
H_{2}\left(G, \mathbb{F}_{p}\right) \rightarrow H_{1}\left(X_{\infty}, \mathbb{F}_{p}\right)_{G} \rightarrow H_{1}\left(\mathscr{G}, \mathbb{F}_{p}\right)
$$

en appliquant la suite exacte d'inflation-restriction à coefficients dans $\mathbb{F}_{p}$ à la suite exacte $0 \rightarrow X_{\infty} \rightarrow \mathscr{G} \rightarrow G \rightarrow 0$.

Le groupe abélien $H_{2}\left(G, \mathbb{F}_{p}\right)$ est de type fini du fait que $G$ est analytique. Ce terme de gauche étant également tué par $p$, il est fini. Concernant le terme de droite, l'extension $H_{S}^{T} / K$ est non-ramifiée en dehors de l'ensemble fini $\Sigma$, composé des places de $S$ et des places ramifiées dans $K_{\infty} / K$. Le groupe $\mathscr{G}$ est donc un quotient de $G_{\Sigma}$ : groupe de Galois de la pro- $p$-extension maximale de $K$ qui est non-ramifiée en dehors des places de $\Sigma$. D'après la théorie du corps de classes $G_{\Sigma}$ est de type fini donc $\mathscr{G}$ aussi. Le terme de droite est ainsi lui aussi fini et on en déduit que le terme médian, $\left(X_{\infty}\right)_{G} / p$, est fini. D'après le lemme de Nakayama (voir [Balister et Howson 1997]), $X_{\infty}$ est de type fini sur $\Lambda$.

Les formules asymptotiques. La théorie du corps de classes interprète le module $X_{S, n}^{T}$ comme le $p$-groupe des $T$-classes $S$-infinitésimales de $K_{n}$ (voir [Jaulent 1986]). Le module $X_{S, \infty}^{T}$ étant construit à partir des $X_{S, n}^{T}$, on retrouve des informations sur les groupes des classes généralisées à l'étage $n$ à partir du module à l'infini. C'est le principe de montée-descente de la théorie d'Iwasawa.

Lorsque $G \simeq \mathbb{Z}_{p}, S=\varnothing$ et $T$ est l'ensemble des places réelles de $K$, ce procédé donne l'un des tous premiers résultats d'Iwasawa dans son étude des $\mathbb{Z}_{p^{-}}$ extensions. Il obtient l'évolution asymptotique du nombre de $p$-classes le long d'une $\mathbb{Z}_{p}$-extension de corps de nombres et fait intervenir les invariants d'Iwasawa du module à l'infini correspondant (voir [Serre 1966]). Ce résultat a été, par la 
suite, généralisé par Jaulent au cas des $p$-groupes des $T$-classes $S$-infinitésimales, toujours le long d'une $\mathbb{Z}_{p}$-extension de corps de nombres [Jaulent 2005].

Dans le cas d'un groupe $p$-valué, le résultat suivant réalise la descente de $X_{S, \infty}^{T}$ à $X_{S, n}^{T}$. Il nous informe que le comportement asymptotique des $G_{n}$-coïnvariants $\left(X_{S, \infty}^{T}\right)_{G_{n}}$ de $X_{S, \infty}^{T}$ est proche de celui des $X_{S, n}^{T}$.

Proposition 3.2. On a

$$
\begin{aligned}
\#\left(\left(X_{S, \infty}^{T}\right)_{G_{n}} / p^{n}\right) & =\#\left(X_{S, n}^{T} / p^{n}\right) p^{O\left(n p^{n(d-1)}\right)}, \\
\operatorname{dim}_{\mathbb{F}_{p}}\left(\left(X_{S, \infty}^{T}\right)_{G_{n}} / p\right) & =\operatorname{dim}_{\mathbb{F}_{p}}\left(X_{S, n}^{T} / p\right)+O\left(p^{n(d-1)}\right), \\
\operatorname{rk}_{\mathbb{Z}_{p}}\left(X_{S, \infty}^{T}\right)_{G_{n}} & =\operatorname{rk}_{\mathbb{Z}_{p}}\left(X_{S, n}^{T}\right)+O\left(p^{n(d-1)}\right) .
\end{aligned}
$$

Démonstration. On oublie les indices $S$ et $T$ dans cette preuve et on se concentre sur la première équivalence.

La suite exacte d'inflation-restriction à coefficients dans $\mathbb{Z} / p^{n} \mathbb{Z}$, appliquée à la suite exacte

$$
0 \rightarrow X_{\infty} \rightarrow \mathscr{G}_{n} \rightarrow G_{n} \rightarrow 0
$$

conduit à

$$
H_{2}\left(G_{n}, \mathbb{Z} / p^{n} \mathbb{Z}\right) \rightarrow\left(X_{\infty}\right)_{G_{n}} / p^{n} \rightarrow \varphi_{n}^{a b} / p^{n} \rightarrow H_{1}\left(G_{n}, \mathbb{Z} / p^{n} \mathbb{Z}\right) \rightarrow 0 .
$$

Le corollaire 2.4 appliqué à $M=\mathbb{Z}_{p}$ nous indique que le cardinal de $H_{i}\left(G_{n}, \mathbb{Z} / p^{n} \mathbb{Z}\right)$ évolue en $p^{O\left(n p^{n(d-1)}\right)}$. On en déduit que

$$
\#\left(\left(X_{\infty}\right)_{G_{n}} / p^{n}\right)=\#\left(\varphi_{n}^{a b} / p^{n}\right) p^{O\left(n p^{n(d-1)}\right)} .
$$

Il reste donc à comparer $\varphi_{n}^{a b}$ à $X_{n}$. On s'appuie sur ce qui est fait dans le théorème 13.13 de [Washington 1997] pour $G \simeq \mathbb{Z}_{p}, S=\varnothing$ et $T$ l'ensemble des places réelles de $K$.

La situation galoisienne nous informe que $X_{n}$ s'obtient comme le quotient de $\varphi_{n}^{a b}$ par son sous-groupe $\mathscr{D}_{n}$, engendré par l'inertie des places hors de $S$ et la décomposition des places de $T$. Soit $D_{v_{n}}$ l'un des sous-groupes d'inertie ou de décomposition non triviaux qui interviennent. Le groupe $D_{v_{n}}$ est alors associé à une place $v_{n}$ de $K_{n}$ au dessus d'une place $v$ de $K$ qui n'est pas totalement décomposée dans $K_{\infty} / K$. On a une injection de $D_{v_{n}}$ dans $G_{n}$, qui nous informe que le nombre minimal de générateurs de $D_{v_{n}}$ est inférieur à $d$ pour $n$ assez grand. Le $p$-rang de $D_{v_{n}}^{a b}$ est donc inférieur à $d$. On en déduit que le $p$-rang de $\mathscr{D}_{n}$ est borné par $d$ fois le cardinal de l'ensemble des places $v_{n}$ que l'on considère. La place $v$ n'étant pas totalement décomposée, le lemme qui suivant de voir que le nombre de places $v_{n}$ au dessus de $v$ évolue en $O\left(p^{n(d-1)}\right)$. 
Lemme 3.3 [Hachimori et Sharifi 2005, lemme 4.2]. Soit $v$ une place de $K$ qui $n$ 'est pas totalement décomposée dans $K_{\infty} / K$. On note $V_{n}$ l'ensemble des places de $K_{n}$ divisant $v$. Alors

$$
\#\left(V_{n}\right)=O\left(p^{n(d-1)}\right) .
$$

Démonstration. On fixe une place $w$ de $K_{\infty}$ au dessus de $v$. La place $v$ n'étant pas totalement décomposée et $G$ étant sans torsion, on en déduit que le sous-groupe de décomposition $G_{v} \subset G$, associé à $w \mid v$, est de dimension $d_{v}>0$. On note $G_{v, n}$ la filtration de $G_{v}$ induite par la filtration $G_{n}$ de $G$. La proposition 1.3 nous informe que, pour $n$ assez grand, il existe des constantes $C$ et $C^{\prime}$ telles que

$$
\left(G: G_{n}\right)=C p^{n d} \quad \text { et } \quad\left(G_{v}: G_{v, n}\right)=C^{\prime} p^{n d_{v}} .
$$

Mais

$$
\#\left(V_{n}\right)=\left(G / G_{n}: G_{v} / G_{v, n}\right)=\frac{C}{C^{\prime}} p^{n\left(d-d_{v}\right)} .
$$

Finalement, comme les places $v$ à considérer sont en nombre fini, on obtient $\#\left(\mathscr{D}_{n} / p^{n}\right)=p^{O\left(n p^{n(d-1)}\right)}$ et

$$
\#\left(\varphi_{n}^{a b} / p^{n}\right)=\#\left(X_{n} / p^{n}\right) p^{O\left(n p^{n(d-1)}\right)} .
$$

La seconde équivalence se démontre en utilisant les mêmes arguments, le corollaire 2.3 remplaçant corollaire 2.4. La preuve de la dernière assertion est similaire, la suite exacte d'inflation-restriction étant à prendre à coefficients dans $\mathbb{Z}_{p}$.

On est alors en mesure de voir que le comportement asymptotique des $p$-groupes des classes $X_{S, n}^{T}$ est contrôlé par les invariants d'Iwasawa de l'extension. Le résultat suivant est le théorème 0.1 de l'introduction :

Corollaire 3.4. Sous les hypothèses de cette section, on a les trois identités:

$$
\begin{aligned}
\operatorname{rk}_{\mathbb{Z}_{p}}\left(X_{S, n}^{T}\right) & =\rho_{S}^{T}\left(G: G_{n}\right)+O\left(p^{n(d-1)}\right), \\
\#\left(X_{S, n}^{T} / p^{n}\right) & =p^{\left(\rho_{S}^{T} n+\mu_{S}^{T}\right)\left(G: G_{n}\right)+O\left(n p^{n(d-1)}\right)}, \\
\operatorname{dim}_{\mathbb{F}_{p}}\left(X_{S, n}^{T} / p\right) & =\left(\rho_{S}^{T}+r_{S}^{T}\right)\left(G: G_{n}\right)+O\left(p^{n(d-1)}\right) .
\end{aligned}
$$

Démonstration. La proposition 3.2 relie les quantités se rapportant à $X_{S, n}^{T}$ aux coïnvariants $\left(X_{S, \infty}^{T}\right)_{G_{n}}$. Les deux dernières assertions découlent alors du théorème 2.1. Pour la première assertion, il faut substituer au théorème 2.1 le théorème $1.10 \mathrm{de}$ [Harris 2000], qui affirme que

$$
\operatorname{rk}_{\mathbb{Z}_{p}}\left(X_{S, \infty}^{T}\right)_{G_{n}}=\rho_{S}^{T}\left(G: G_{n}\right)+O\left(p^{n(d-1)}\right) .
$$


Les formules de réflexion. A l'imitation de ce qui est fait dans [Jaulent 2005], [Jaulent et Maire 2003] ou [Maire 2005], on va utiliser les théorèmes de réflexion de Gras [1998, 2003] pour obtenir des informations sur les invariants relatifs au couple $(S, T)$ par rapport à ceux relatifs au couple $(T, S)$, lorsque $S \cup T$ contient l'ensemble des places $p$-adiques de $K$. Ces informations traduisent une dualité entre la ramification et la décomposition. La théorie de Kummer joue un rôle central dans les formules de réflexion, c'est pourquoi la présence de racines de l'unité est nécessaire.

On introduit les notations suivantes :

Définition 3.5. On note $\mathrm{Pl}_{p}$ l'ensemble des places $p$-adiques de $K$ et $\mathrm{Pl}_{\infty}^{\mathbb{R}}$ celui de ses places réelles.

Pour $S$ un ensemble fini de places de $K$, on note $S_{0}$ l'ensemble des places finies de $S, S_{\infty}$ l'ensemble de ses places infinies et $S_{p}$ l'ensemble de ses places $p$-adiques.

- On note $s_{\infty}^{\mathbb{R}}$ le nombre de places réelles de $S$ et $s_{0}$ le nombre de places finies de $S$. Si $S$ ne contient que des places finies, on notera $s$ à la place de $s_{0}$.

- On note $s_{0}^{\text {dec }}$ le nombre de places de $S_{0}$ totalement décomposées dans $K_{\infty} / K$. On le notera aussi $s^{\text {dec }}$ si $S$ ne contient que des places finies.

- On pose $\delta_{S}=\sum_{v \in S_{p}}\left[K_{v}: \mathbb{Q}_{p}\right]$ le degré $p$-adique en $S$.

La formule de réflexion sur laquelle on se base dans un premier temps est la suivante :

Théorème 3.6 [Gras 2003, théorème I.4.6]. Soit $p$ un premier et $K$ un corps de nombres contenant $\mu_{p}$, de signature $\left(r_{1}, r_{2}\right)$. On se donne $S$ et $T$ deux ensembles finis disjoints de places de $K$ vérifiant $\mathrm{Pl}_{p} \cup \mathrm{Pl}_{\infty}^{\mathbb{R}} \subset S \cup T$. On a alors la formule suivante, concernant les groupes des classes généralisées associés à $K$ :

$$
\operatorname{dim}_{\mathbb{F}_{p}}\left(X_{S}^{T} / p\right)-\operatorname{dim}_{\mathbb{F}_{p}}\left(X_{T}^{S} / p\right)=s_{0}-t_{0}+\delta_{S}-r_{1}-r_{2}+\delta_{2, p} s_{\infty}^{\mathbb{R}},
$$

où $\delta_{2, p}=1$ si $p=2$ et 0 sinon.

Cas $p=2$. On commence par traiter le cas $p=2$. On remarque tout d'abord que les places à l'infini n'ont aucune incidence sur l'invariant $\rho$.

Proposition 3.7.

$$
\rho_{S}^{T}=\rho_{S_{0}}^{T_{0}} \text {. }
$$

Démonstration. Il est clair que $X_{S, \infty}^{T}=X_{S_{0}, \infty}^{T \cup \mathrm{Pl}_{\infty}-S_{\infty}}$. La théorie du corps de classes donne alors une suite exacte

$$
\bigoplus_{T_{\infty} \cup \mathrm{Pl}_{\infty}-S_{\infty}} \mathbb{Z} / 2 \mathbb{Z} \rightarrow X_{S_{0}, \infty}^{T_{0}} \rightarrow X_{S_{0}, \infty}^{T \cup \mathrm{Pl}_{\infty}-S_{\infty}} \rightarrow 0
$$

qui conduit au résultat car le premier terme est de $\Lambda$-torsion.

Les invariants d'Iwasawa de l'extension $K_{\infty} / K$ vérifient : 
Théorème 3.8. Pour $p=2$ et $\mathrm{Pl}_{p} \cup \mathrm{Pl}_{\infty}^{\mathbb{R}} \subset S \cup T$, on $a$

$$
\rho_{S}^{T}+r_{S}^{T}+\frac{\delta_{T}}{2}+t_{0}^{\mathrm{dec}}+\frac{t_{\infty}^{\mathbb{R}}}{2}=\rho_{T}^{S}+r_{T}^{S}+\frac{\delta_{S}}{2}+s_{0}^{\mathrm{dec}}+\frac{s_{\infty}^{\mathbb{R}}}{2} .
$$

Démonstration. Le théorème 3.6 nous indique que pour tout $n$,

$$
\begin{aligned}
\operatorname{dim}_{\mathbb{F}_{p}}\left(X_{S, n}^{T} / p\right)-\operatorname{dim}_{\mathbb{F}_{p}}\left(X_{T, n}^{S} / p\right) & \\
& =s_{0}\left(K_{n}\right)-t_{0}\left(K_{n}\right)+\delta_{S}\left(K_{n}\right)-r_{1}\left(K_{n}\right)-r_{2}\left(K_{n}\right)+s_{\infty}^{\mathbb{R}}\left(K_{n}\right) .
\end{aligned}
$$

Il faut remarquer ici que l'on a

$$
\begin{aligned}
r_{1}\left(K_{n}\right)+r_{2}\left(K_{n}\right) & =\frac{1}{2}\left(r_{1}\left(K_{n}\right)+2 r_{1}\left(K_{n}\right)+r_{1}\left(K_{n}\right)\right) \\
& =\frac{1}{2}\left(\delta_{S}\left(K_{n}\right)+\delta_{T}\left(K_{n}\right)+s_{\infty}^{\mathbb{R}}\left(K_{n}\right)+t_{\infty}^{\mathbb{R}}\left(K_{n}\right)\right) .
\end{aligned}
$$

Le passage à la deuxième ligne se justifie grâce à l'hypothèse $\mathrm{Pl}_{p} \cup \mathrm{Pl}_{\infty}^{\mathbb{R}} \subset S \cup T$. La quantité $\operatorname{dim}_{\mathbb{F}_{p}}\left(X_{S, n}^{T} / p\right)-\operatorname{dim}_{\mathbb{F}_{p}}\left(X_{T, n}^{S} / p\right)$ est alors égale à

$$
s_{0}\left(K_{n}\right)-t_{0}\left(K_{n}\right)+\frac{\delta_{S}\left(K_{n}\right)}{2}-\frac{\delta_{T}\left(K_{n}\right)}{2}+\frac{s_{\infty}^{\mathbb{R}}\left(K_{n}\right)}{2}-\frac{t_{\infty}^{\mathbb{R}}\left(K_{n}\right)}{2} .
$$

Asymptotiquement, on a

$$
\delta_{S}\left(K_{n}\right)=\delta_{S}\left(G: G_{n}\right) \quad \text { et } \quad s_{0}\left(K_{n}\right)=s_{0}^{\operatorname{dec}}\left(G: G_{n}\right)+O\left(p^{n(d-1)}\right),
$$

car le lemme 3.3 permet de négliger les places qui ne sont pas totalement décomposées. Le groupe $G$ est sans torsion donc les places de $S_{\infty}$ sont totalement décomposées dans $K_{\infty} / K$. On en déduit que $s_{\infty}^{\mathbb{R}}\left(K_{n}\right)=s_{\infty}^{\mathbb{R}}\left(G: G_{n}\right)$. Les mêmes considérations pour $T$ conduisent à la formule

$$
\begin{aligned}
\operatorname{dim}_{\mathbb{F}_{p}}\left(X_{S, n}^{T} / p\right)- & \operatorname{dim}_{\mathbb{F}_{p}}\left(X_{T, n}^{S} / p\right) \\
& =\left(s_{0}^{\mathrm{dec}}-t_{0}^{\mathrm{dec}}+\frac{\delta_{S}}{2}-\frac{\delta_{T}}{2}+\frac{s_{\infty}^{\mathbb{R}}}{2}-\frac{t_{\infty}^{\mathbb{R}}}{2}\right)\left(G: G_{n}\right)+O\left(p^{n(d-1)}\right) .
\end{aligned}
$$

On obtient la formule de l'énoncé en comparant avec le comportement asymptotique de $\operatorname{dim}_{\mathbb{F}_{p}}\left(X_{S, n}^{T} / p\right)-\operatorname{dim}_{\mathbb{F}_{p}}\left(X_{T, n}^{S} / p\right)$ donné par le corollaire 3.4.

Cas $p \neq 2$ et $\mu_{p} \subset K$. La complexification des places réelles ne produit que de la 2-torsion dans les $p$-groupes $X_{S}^{T}$. Comme $p$ est différent de 2 dans cette section les places réelles ne jouent aucun rôle. On peut donc considérer ici que $S$ et $T$ contiennent uniquement des places finies.

Dans ce paragraphe, on fait l'hypothèse que $\mu_{p}$, le groupe des racines $p$-ièmes de l'unité, est inclu dans $K$. Le corps $K$ est alors totalement imaginaire et on obtient : 
Théorème 3.9. Lorsque $\mu_{p} \subset K$ avec $p$ impair et sous l'hypothèse que $\mathrm{Pl}_{p} \subset S \cup T$, on a la formule suivante concernant les invariants $\rho$ et $r$ :

$$
\rho_{S}^{T}+r_{S}^{T}+\frac{\delta_{T}}{2}+t^{\mathrm{dec}}=\rho_{T}^{S}+r_{T}^{S}+\frac{\delta_{S}}{2}+s^{\mathrm{dec}} .
$$

Démonstration. Sous ces hypothèses, le terme de droite de la formule du théorème 3.6 devient

$$
s\left(K_{n}\right)-t\left(K_{n}\right)+\delta_{S}\left(K_{n}\right)-\frac{1}{2}\left[K_{n}: \mathbb{Q}\right] .
$$

La relation $\left[K_{n}: \mathbb{Q}\right]=\delta_{S}\left(K_{n}\right)+\delta_{T}\left(K_{n}\right)$, provenant de l'hypothèse $\mathrm{Pl}_{p} \subset S \cup T$, ainsi que le lemme 3.3, qui permet de négliger les places qui ne sont pas totalement décomposées, donnent alors l'évolution

$\operatorname{dim}_{\mathbb{F}_{p}}\left(X_{S, n}^{T} / p\right)-\operatorname{dim}_{\mathbb{F}_{p}}\left(X_{T, n}^{S} / p\right)=\left(s^{\mathrm{dec}}-t^{\mathrm{dec}}+\frac{\delta_{S}}{2}-\frac{\delta_{T}}{2}\right)\left(G: G_{n}\right)+O\left(p^{n(d-1)}\right)$.

On conclut en invoquant le corollaire 3.4, qui donne l'évolution asymptotique de $\operatorname{dim}_{\mathbb{F}_{p}}\left(X_{S, n}^{T} / p\right)-\operatorname{dim}_{\mathbb{F}_{p}}\left(X_{T, n}^{S} / p\right)$.

Lorsque l'extension $K_{\infty} / K$ contient toutes les racines $p$-primaires de l'unité, les informations obtenues seront plus précises. On traite donc ce cas dans le paragraphe suivant.

Cas cyclotomique. On suppose toujours dans cette section que $\mu_{p} \subset K\left(\mu_{4} \subset K\right.$ si $p=2$ ), et également que $K_{\infty} / K$ contient la $\mathbb{Z}_{p}$-extension cyclotomique de $K$, soit $K\left(\mu_{p}\right) \subset K_{\infty}$.

Ces hypothèses assurent que, pour $k$ assez grand, le corps $K_{k+n}$ contient les racines $p^{n}$-ièmes de l'unité. En effet, $G$ est produit semi-direct du groupe de Galois de la $\mathbb{Z}_{p}$-extension cyclotomique de $K$, noté $\Gamma$, par un sous-groupe distingué $H$ de $G$. Prenons $k$ assez grand pour que $G_{k}$ soit $p$-saturé, de telle sorte que $G_{k+n}=G_{k}^{p^{n}}$. Pour tout $n$, les racines $p^{n}$-ièmes de l'unité sont fixées par $H$ et par $\Gamma^{p^{n}}$, donc par $G_{k}^{p^{n}}$. Autrement dit, $\mu_{p^{n}} \subset K_{k+n}$. On fixe cette constante $k$ dans la suite, en remarquant qu'elle est nulle dans le cas d'un groupe uniforme.

On aura besoin d'un certain nombre de notations qui servirons à énoncer le théorème fondamental de cette partie, qui exprime une lien entre le groupe des classes associé à la $S$-ramification, $T$-décomposition d'une part, et le radical kummerien associé à la $T$-ramification, $S$-décomposition d'autre part.

On pourra se référer à [Jaulent 1998] pour les notations de la théorie $p$-adique du corps de classes. La notation $\mathfrak{m}$ vaut pour un certain diviseur, construit sur les places de $S\left(K_{k+n}\right)$. Le module $\mathscr{R}$ est le $p$-adifié des idéles principaux de $K_{k+n}$ et $\mathscr{R}_{\mathfrak{m}}$ est son sous-module des idéles congrues à 1 modulo $\mathfrak{m}$. On appelle $\mathscr{E}_{\mathfrak{m}}^{T}$ le $p$-adifié des $T$-unités de $K_{k+n}$ qui sont congrues à 1 modulo $\mathfrak{m}$. Comme expliqué dans [Jaulent et Maire 2003], le diviseur $\mathfrak{m}$ a été choisi de telle sorte que le quotient d'ordre $p^{n}$ du $p$-groupe des $T$-classes de rayon $\mathfrak{m}$ de $K_{k+n}$, noté $\mathrm{Cl}_{\mathfrak{m}}^{T}$, 
soit isomorphe au quotient d'ordre $p^{n}$ du groupe de Galois de sa pro- $p$-extension abélienne $S$-ramifiée $T$-décomposée maximale $X_{S}^{T}$, et que le radical kummérien du quotient de l'extension duale, $T$-ramifiée et $S$-décomposée, soit donné par $\operatorname{Rad}_{\mathfrak{m}}^{T}$. Enfin, $\mathfrak{R}_{\mathfrak{m}}^{T}\left[p^{n}\right]$ est un pseudo-radical qui permet de faire le lien entre les deux suites du théorème suivant et qui est défini par

$$
\mathfrak{R}_{\mathfrak{m}}^{T}\left[p^{n}\right]=\left\{p^{n} \otimes x \in p^{-n} \mathbb{Z}_{p} / \mathbb{Z}_{p} \otimes \mathscr{R}_{\mathfrak{m}} \mid x \in \mathscr{F}^{T} \mathscr{g}^{p^{n}}\right\},
$$

où $\mathscr{F}$ est le $p$-groupe des idèles de $K_{k+n}$ et $\mathscr{g}^{T}$ son sous-groupe des $T$-idèles.

Théorème 3.10 [Jaulent et Maire 2003, théorème 4]. A chaque étage $K_{k+n}$ de la tour $K_{\infty} / K$, on a les suites exactes du miroir:

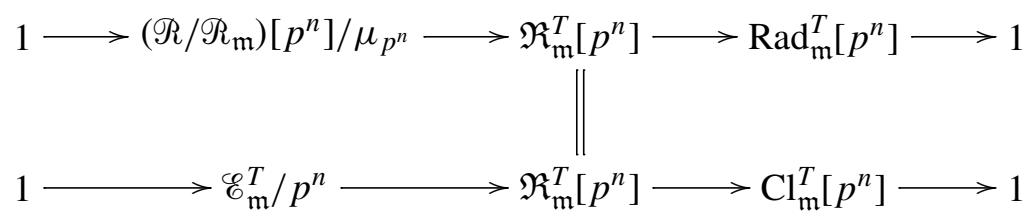

Le corollaire suivant résulte du calcul des cardinaux des termes des suites exactes du miroir, conjointement au corollaire 3.4. Il donne le théorème 0.2 annoncé en introduction.

Corollaire 3.11. On suppose que $\mu_{p} \subset K$ et que $K_{\infty}$ contient la $\mathbb{Z}_{p}$-extension cyclotomique de $K$. On a les formules suivantes, lorsque $\mathrm{Pl}_{p} \subset S \cup T$, concernant les invariants associés à l'extension $K_{\infty} / K$ :

$$
\rho_{S}^{T}+\frac{\delta_{T}}{2}=\rho_{T}^{S}+\frac{\delta_{S}}{2}, \quad r_{S}^{T}=r_{T}^{S} \quad \text { et } \quad \mu_{S}^{T}=\mu_{T}^{S} .
$$

Démonstration. Dans cette démonstration, les modules qui interviennent sont attachés au corps $K_{k+n}$.

- Tout d'abord, on fait apparaître les invariants d'Iwasawa en regardant les cardinaux des termes de droite des deux suites. En effet, $\mathrm{Cl}_{\mathfrak{m}}^{T}$ étant un groupe fini, on en déduit, d'après la théorie du corps de classes, que

$$
\#\left(\mathrm{Cl}_{\mathfrak{m}}^{T}\left[p^{n}\right]\right)=\#\left(\mathrm{Cl}_{\mathfrak{m}}^{T} / p^{n}\right)=\#\left(\mathrm{Cl}_{S}^{T} / p^{n}\right)=\#\left(X_{S}^{T} / p^{n}\right) .
$$

D'autre part, utilisant la théorie de Kummer,

$$
\#\left(\operatorname{Rad}_{\mathfrak{m}}^{T}\left[p^{n}\right]\right)=\#\left(\operatorname{Rad}_{S}^{T}\left[p^{n}\right]\right)=\#\left(\operatorname{Hom}\left(X_{T}^{S} / p^{n}, \mu_{p^{n}}\right)\right)=\#\left(X_{T}^{S} / p^{n}\right) .
$$

Les arguments conduisant au corollaire 3.4 peuvent être modifiés pour tenir compte du décalage de $k$ et conduisent à la formule :

$$
\#\left(X_{S, k+n}^{T} / p^{n}\right)=p^{\left(\rho_{S}^{T} n+\mu_{S}^{T}\right)\left(G: G_{k+n}\right)+O\left(n p^{n(d-1)}\right)} .
$$


Les invariants $\rho_{S}^{T}, \mu_{S}^{T}$ et $\rho_{T}^{S}, \mu_{T}^{S}$ interviennent donc dans les cardinaux de $\mathrm{Cl}_{\mathfrak{m}}^{T}\left[p^{n}\right]$ et de $\operatorname{Rad}_{\mathfrak{m}}^{T}\left[p^{n}\right]$ respectivement.

Il reste à calculer les cardinaux des termes de gauche. On s'appuie sur ce qui est fait dans [Jaulent et Maire 2003].

- Le lemme d'approximation nous permet de voir que $\left(\mathscr{R} / \mathscr{R}_{\mathfrak{m}}\right)\left[p^{n}\right] \simeq u^{S} / p^{n}$, où $U^{S}$ désigne le produit sur les places de $S\left(K_{k+n}\right)$ des complétés $p$-adiques des unités locales :

$$
u^{S} / p^{n} \simeq \prod_{v \in S_{p}\left(K_{k+n}\right)} \mathbb{Z} / p^{n} \mathbb{Z}^{\left[K_{k+n, v}: \mathbb{Q}_{p}\right]} \prod_{v \in S\left(K_{k+n}\right)} \mu_{p^{n}}
$$

La partie racines de l'unité contribue en $p^{O\left(n p^{n(d-1)}\right)}$. En effet, comme $K_{\infty} / K$ contient la $\mathbb{Z}_{p}$-extension cyclotomique, aucune place n'est totalement décomposée dans $K_{\infty} / K$. On fait alors appel au lemme 3.3 pour conclure que $\#\left(S\left(K_{k+n}\right)\right)=$ $O\left(p^{n(d-1)}\right)$.

Quant au produit restant, son cardinal est donné par $p^{\delta_{S} n\left(G: G_{k+n}\right)}$. Le quotient par les racines globales de l'unité ne change rien (cardinal en $p^{O(n)}$ ) et on en déduit que

$$
\#\left(\left(\mathscr{R} / \mathscr{R}_{\mathfrak{m}}\right)\left[p^{n}\right] / \mu_{p^{n}}\right)=p^{\delta_{S} n\left(G: G_{k+n}\right)+O\left(n p^{n(d-1)}\right)} .
$$

- Le dernier cardinal à calculer et celui de $\mathscr{E}_{\mathfrak{m}}^{T} / p^{n}$. On a $\#\left(\mathscr{E}_{\mathfrak{m}} T / p^{n}\right)=\#\left(\mathscr{E}^{T} / p^{n}\right) p^{O(n)}$ car les $\mathbb{Z}_{p}$-modules $\mathscr{E}^{T}$ et $\mathscr{E}_{\mathfrak{m}}^{T}$ sont de même rang (pour vérifier cela on peut regarder l'injection $\mathscr{E}^{T} / \mathscr{E}_{\mathfrak{m}}^{T} \hookrightarrow \prod_{v \mid \mathfrak{m}} U_{v} / U_{v}^{\mathfrak{m}_{v}}$ ) et leur torsion est composée de racines globales de l'unité, dont le cardinal évolue en $p^{O(n)}$. On trouve le cardinal voulu en appliquant le théorème de Dirichlet sur les $T$-unités qui donne

$$
\#\left(\mathscr{E}^{T} / p^{n}\right)=p^{\frac{1}{2}[K: \mathbb{Q}] n\left(G: G_{k+n}\right)+O\left(n p^{n(d-1)}\right)} .
$$

- Finalement, mettant ces calculs ensemble, on trouve les formules

$$
\rho_{S}^{T}+\frac{1}{2}[K: \mathbb{Q}]=\rho_{T}^{S}+\delta_{S} \quad \text { et } \quad \mu_{S}^{T}=\mu_{T}^{S} .
$$

La formule symétrique de l'énoncé concernant $\rho$ provient alors de l'hypothèse $\mathrm{Pl}_{p} \subset S \cup T$, qui implique $[K: \mathbb{Q}]=\delta_{S}+\delta_{T}$.

- En recoupant avec les théorèmes 3.8 et 3.9, on obtient l'égalité entre $r_{S}^{T}$ et $r_{T}^{S}$.

On peut aussi déduire de ce résultat une formule de réflexion concernant le $\mathbb{Z}_{p^{-}}$ rang des modules $X_{S, n}^{T}$

Corollaire 3.12. Sous les mêmes hypothèses, on a

$$
\operatorname{rk}_{\mathbb{Z}_{p}}\left(X_{T, n}^{S}\right)-\operatorname{rk}_{\mathbb{Z}_{p}}\left(X_{S, n}^{T}\right)=\left(\frac{\delta_{T}}{2}-\frac{\delta_{S}}{2}\right)\left(G: G_{n}\right)+O\left(p^{n(d-1)}\right) .
$$

Démonstration. Le corollaire 3.11 donne un lien entre $\rho_{S}^{T}$ et $\rho_{T}^{S}$. On sait, d'après le corollaire 3.4, que l'invariant $\rho$ contrôle le $\mathbb{Z}_{p}$-rang de $X_{n}$, d'où le résultat. 
Calcul des invariants. Dans [Jaulent et Maire 2003], les formules de réflexion débouchent sur le calcul de l'invariant $\rho_{S}^{T}$ dans le cas CM, ainsi que sur des inégalités concernant les invariants $\mu_{S}^{T}$ lorsque $S$ et $T$ varient. Nous allons exhiber des résultats de ce type dans le cadre non-commutatif.

On suppose dans cette section que $p \neq 2$ ou que $K$ est totalement imaginaire. On fait aussi l'hypothèse que $K_{\infty} / K$ contient la $\mathbb{Z}_{p}$-extension cyclotomique, ce qui assure les deux propriétés essentielles suivantes :

- Aucune place finie de $K$ n'est totalement décomposée dans $K_{\infty} / K$.

- L'extension $K_{\infty} / K$ vérifie la condition de Leopoldt faible.

La condition de Leopoldt faible exprime que le groupe d'homologie

$$
H_{2}\left(G_{S}\left(K_{\infty}\right), \mathbb{Z}_{p}\right)
$$

est nul dès lors que $S$ contient les places $p$-adiques, avec $G_{S}\left(K_{\infty}\right)$ qui désigne le groupe de Galois de la pro- $p$-extension $S$-ramifiée maximale de $K_{\infty}$. Il est démontré dans [Nguyen-Quang-Do 1984] que cette condition est vérifiée en présence de la $\mathbb{Z}_{p}$-extension cyclotomique.

On va utiliser une formulation équivalente de cette condition, en terme de rang d'unités, que l'on peut trouver dans [Maire 2011, proposition 4.9]. On donne ici les grandes lignes de la démonstration. Pour un ensemble fini $S$ de places de $K$ contenant les places $p$-adiques et les places ramifiées dans $K_{\infty} / K$, on note $K_{S}$ la pro- $p$-extension maximale $S$-ramifiée de $K$ et $G_{S}(K)=\mathrm{Gal}\left(K_{S} / K\right)$. Le corps $K_{\infty}$ est alors inclu dans $K_{S}$ et on note $X_{S}=\operatorname{Gal}\left(K_{S} / K_{\infty}\right)^{a b}=G_{S}\left(K_{\infty}\right)^{a b}$. La condition $H_{2}\left(G_{S}\left(K_{\infty}\right), \mathbb{Z}_{p}\right)=0$ permet de relier le $\Lambda$-rang de $X_{S}$ à la caractéristique d'Euler-Poincaré de $G_{S}(K)$ qui est connue. On en déduit ensuite une formule pour $\operatorname{rk}_{\mathbb{Z}_{p}}\left(X_{S, n}\right)$ grâce à la première formule du corollaire 3.4, que l'on relie au défaut de Leopoldt $\mathscr{E}_{S, n}=\operatorname{ker}\left(\mathscr{E}_{n} \rightarrow \mathcal{U}_{n}^{S}\right)$ via la théorie du corps de classes. On rappelle que $\mathscr{E}_{n}$ est le tensorisé par $\mathbb{Z}_{p}$ du groupe des unités globales de $K_{n}$ et que $u_{n}^{S}$ désigne le produit des $p$-adifiés des unités locales en toutes les $S$-places de $K_{n}$ (voir les notations avant le théorème 3.10).

On en déduit l'expression suivante de la condition de Leopoldt faible,

$$
\operatorname{rk}_{\mathbb{Z}_{p}}\left(\mathscr{E}_{S, n}\right)=O\left(p^{n(d-1)}\right) .
$$

Cette condition est bien évidemment vérifiée si on admet la conjecture de Leopoldt pour les étages $K_{n}$.

Indépendance en $T$ et en $S-S_{p}$. Dans le cas de la $\mathbb{Z}_{p}$-extension cyclotomique, les invariants $\rho_{S}^{T}$ et $\mu_{S}^{T}$ ne dépendent que de $S_{p}$. L'argument, provenant de la théorie du corps de classes, est que les $p$-adifiés des sous-groupes de décomposition des places au-dessus de $T$ et d'inertie des places de $S-S_{p}$ dans $X_{S, \infty}$ sont des $\mathbb{Z}_{p^{-}}$ modules de rang au plus 1 . C'est toujours vrai dans le cas général mais les places 
à considérer sont alors en nombre infini ce qui empêche de conclure. Il faut alors redescendre à $X_{S, n}^{T}$ pour démontrer :

Théorème 3.13. $\quad \rho_{S}^{T}=\rho_{S_{p}}, \quad \mu_{S}^{T}=\mu_{S_{p}} \quad$ et $\quad r_{S}^{T}=r_{S_{p}}$.

Démonstration. Comme remarqué juste avant, les sous-groupes de décomposition et d'inertie des places de $T \cup S-S_{p}$ dans $X_{S, n}$ sont de $p$-rang au plus 1 . Ces sous-groupes sont asymptotiquement au nombre de $O\left(p^{n(d-1)}\right)$ car les places en question ne sont pas totalement décomposées dans $K_{\infty} / K$ (lemme 3.3). On peut conclure dans ce cas que

$$
\#\left(X_{S, n}^{T} / p^{n}\right)=\#\left(X_{S_{p}, n} / p^{n}\right) p^{O\left(n p^{n(d-1)}\right)}
$$

et que

$$
\operatorname{dim}_{\mathbb{F}_{p}}\left(X_{S, n}^{T} / p\right)=\operatorname{dim}_{\mathbb{F}_{p}}\left(X_{S_{p}, n} / p\right)+O\left(p^{n(d-1)}\right) .
$$

Le corollaire 3.4 permet d'en déduire les égalités annoncées.

Calcul de $\rho_{S}^{T}$. On calcule l'invariant $\rho_{S}^{T}$ lorsque les corps $K_{n}$ sont totalement réels ou $\mathrm{CM}$ pour tout $n$, toujours en présence de la $\mathbb{Z}_{p}$-extension cyclotomique.

Pour le cas CM, on note $\tau$ la conjugaison complexe et $\hat{S}=S \cap S^{\tau}$. On a :

Théorème 3.14. (i) Si $K_{\infty}$ est totalement réel, alors

$$
\rho_{S}^{T}=0 .
$$

(ii) Si $K_{n}$ est CM pour tout $n$, alors

$$
\rho_{S}^{T}=\frac{\delta_{\hat{S}}}{2}
$$

Démonstration. Le théorème 3.13 permet de se limiter au calcul de $\rho_{S}$ pour $S$ un ensemble de places $p$-adique de $K$.

On obtient des informations sur $\rho_{S}$ à l'aide de la formule

$$
\operatorname{rk}_{\mathbb{Z}_{p}}\left(X_{S, n}\right)=\rho_{S}\left(G: G_{n}\right)+O\left(p^{n(d-1)}\right)
$$

du corollaire 3.4. La théorie du corps de classes relie cette quantité à un problème de plongement d'unités grâce à la suite exacte

$$
\mathscr{\mathscr { E }}_{n} \rightarrow \mathcal{U}_{n}^{S} \rightarrow X_{S, n} \rightarrow X_{n} \rightarrow 0 .
$$

Les modules $X_{n}$ sont les $p$-groupes des classes classiques donc sont de $\mathbb{Z}_{p}$-torsion. On en déduit

$$
\operatorname{rk}_{\mathbb{Z}_{p}}\left(X_{S, n}\right)=\operatorname{rk}_{\mathbb{Z}_{p}}\left(\operatorname{coker}\left(\mathscr{E}_{n} \rightarrow U_{n}^{S}\right)\right) .
$$

C'est ce dernier conoyau que l'on va calculer. 
(i) On commence par traiter le cas totalement réel. On a

$$
\operatorname{rk}_{\mathbb{Z}_{p}}\left(\operatorname{coker}\left(\mathscr{E}_{n} \rightarrow U_{n}^{S}\right)\right) \leqslant \operatorname{rk}_{\mathbb{Z}_{p}}\left(\operatorname{coker}\left(\mathscr{E}_{n} \rightarrow u_{n}^{\mathrm{Pl}_{p}}\right)\right) \text {. }
$$

Asymptotiquement, le $\mathbb{Z}_{p}$-rang du noyau de cette dernière application évolue en $O\left(p^{n(d-1)}\right)$ d'après la condition de Leopoldt. Comme les corps $K_{n}$ sont totalement réels, $\operatorname{rk}_{\mathbb{Z}_{p}}\left(\mathscr{E}_{n}\right)=[K: \mathbb{Q}]\left(G: G_{n}\right)-1$ d'après le théorème de Dirichlet et $\operatorname{rk}_{\mathbb{Z}_{p}}\left(\mathcal{U}_{n}^{\mathrm{Pl}}{ }_{p}\right)=[K: \mathbb{Q}]\left(G: G_{n}\right)$. On en tire que le $\mathbb{Z}_{p}$-rang de ce dernier conoyau évolue en $O\left(p^{n(d-1)}\right)$, donc que $\rho_{S}=0$.

(ii) On suppose maintenant que $K_{n}$ est CM pour tout $n$ et on commence par traiter le cas où $S$ est stable par $\tau$.

La composante $\mathbb{Z}_{p}$-libre de l'image de $\mathscr{E}_{n} \rightarrow \mathcal{U}_{n}^{S}$ est contenue dans la partie + des unités locales. On a ici

$$
\operatorname{rk}_{\mathbb{Z}_{p}}\left(\mathscr{E}_{n}\right)=r_{2}\left(G: G_{n}\right)-1 \quad \text { et } \quad \operatorname{rk}_{\mathbb{Z}_{p}}\left(\cup_{n}^{\mathrm{Pl}_{p}}\right)^{+}=r_{2}\left(G: G_{n}\right) .
$$

La condition de Leopoldt permet de conclure que

$$
\begin{aligned}
\operatorname{rk}_{\mathbb{Z}_{p}}\left(\operatorname{coker}\left(\mathscr{E}_{n} \rightarrow\left(\mathcal{U}_{n}^{S}\right)^{+}\right)\right) & \leqslant \operatorname{rk}_{\mathbb{Z}_{p}}\left(\operatorname{coker}\left(\mathscr{E}_{n} \rightarrow\left(\mathcal{U}_{n}^{\mathrm{Pl}_{p}}\right)^{+}\right)\right) \\
& =O\left(p^{n(d-1)}\right) .
\end{aligned}
$$

On a alors

$$
\begin{aligned}
\operatorname{rk}_{\mathbb{Z}_{p}}\left(\operatorname{coker}\left(\mathscr{E}_{n} \rightarrow \mathcal{U}_{n}^{S}\right)\right) & =\operatorname{rk}_{\mathbb{Z}_{p}}\left(U_{n}^{S}\right)^{-}+O\left(p^{n(d-1)}\right) \\
& =\frac{\delta_{S}}{2}\left(G: G_{n}\right)+O\left(p^{n(d-1)}\right) .
\end{aligned}
$$

Ainsi, $\rho_{S}=\delta_{S} / 2$.

A partir de maintenant, on ne suppose plus que $S$ est stable par $\tau$. Pour une place $v$ d'un corps CM, l'action de $\tau$ nous informe qu'une unité d'image triviale dans $U_{v}$ est aussi d'image triviale dans $U_{v^{\tau}}$. Notons $\breve{S}=S \cup S^{\tau}$ de telle sorte que pour tout $n$,

$$
\mathscr{E}_{S, n}=\operatorname{ker}\left(\mathscr{E}_{n} \rightarrow u_{n}^{S}\right) \quad \text { et } \quad \mathscr{E}_{\check{S}, n}=\operatorname{ker}\left(\mathscr{E}_{n} \rightarrow u_{n}^{\check{S}_{n}}\right)
$$

sont égaux.

Le diagramme suivant permet de comparer coker $\left(\mathscr{E}_{n} \rightarrow U_{n}^{S}\right)$ à coker $\left(\mathscr{E}_{n} \rightarrow u_{n}^{\check{S}}\right)$, qui est connu car $\check{S}$ est stable $\operatorname{par} \tau$ :

$$
\begin{gathered}
0 \longrightarrow \mathscr{E}_{S, n} \longrightarrow \mathscr{E}_{n} \longrightarrow u_{n}^{S} \longrightarrow \operatorname{coker}\left(\mathscr{E}_{n} \rightarrow u_{n}^{S}\right) \longrightarrow 0 \\
0 \longrightarrow \mathscr{E}_{\check{S}, n} \longrightarrow \mathscr{E}_{n} \longrightarrow u_{n}^{\check{S}} \longrightarrow \operatorname{coker}\left(\mathscr{E}_{n} \rightarrow u_{n}^{\check{S}}\right) \longrightarrow 0 .
\end{gathered}
$$


On a donc

$$
\begin{aligned}
\operatorname{rk}_{\mathbb{Z}_{p}}\left(\operatorname{coker}\left(\mathscr{E}_{n} \rightarrow u_{n}^{S}\right)\right) & =\operatorname{rk}_{\mathbb{Z}_{p}}\left(u_{n}^{S}\right)-\operatorname{rk}_{\mathbb{Z}_{p}}\left(\cup_{n}^{\check{S}}\right)+\operatorname{rk}_{\mathbb{Z}_{p}}\left(\operatorname{coker}\left(\mathscr{E}_{n} \rightarrow u_{n}^{\check{S}}\right)\right) \\
& =\left(\delta_{S}-\delta_{\check{S}}+\frac{\delta_{\check{S}}}{2}\right)\left(G: G_{n}\right)+O\left(p^{n(d-1)}\right) \\
& =\left(\frac{\delta_{\hat{S}}-\delta_{\check{S}}}{2}+\frac{\delta_{\check{S}}}{2}\right)\left(G: G_{n}\right)+O\left(p^{n(d-1)}\right) \\
& =\frac{\delta_{\hat{S}}}{2}\left(G: G_{n}\right)+O\left(p^{n(d-1)}\right),
\end{aligned}
$$

d'où $\rho_{S}=\delta_{\hat{S}} / 2$.

Calcul de $\mu_{S}^{T}$ et $r_{S}^{T}$. Les calculs sur l'invariant $\mu$ contenus dans [Jaulent et Maire 2003] sont encore valables ici et peuvent être étendus à $r$. Nous les retraçons succintement, les démonstrations étant identiques à celles dans le cas de la $\mathbb{Z}_{p}$-extension cyclotomique.

On se place dans le cadre cyclotomique, c'est-à-dire que l'on suppose que $K$ contient les racines $p$-ièmes de l'unité $\left(\mu_{4}\right.$ si $\left.p=2\right)$ et que $K_{\infty} / K$ contient la $\mathbb{Z}_{p}$-extension cyclotomique. Cette condition est ici nécessaire pour obtenir des informations sur $\mu$ et $r$ via les formules de réflexion.

Proposition 3.15. Sous ces hypothèses, on a:

$$
\mu_{S}^{T}=\mu_{S_{p}}=\mu_{\mathrm{Pl}_{p}-S_{p}} \quad \text { et } \quad r_{S}^{T}=r_{S_{p}}=r_{\mathrm{Pl}_{p}-S_{p}} .
$$

(ii) Si de plus $K_{n}$ est $C M$ pour tout $n$ et $S_{p}$ est stable par conjugaison complexe, alors

$$
\mu_{S}^{T} \leqslant \mu \quad \text { et } \quad r_{S}^{T} \leqslant r
$$

où $\mu$ et $r$ sont les invariants correspondant au cas $S=T=\varnothing$.

Démonstration. (i) On utilise le théorème 3.13 et le corollaire 3.11 pour écrire :

$$
\mu_{S}^{T}=\mu_{S_{p}}=\mu_{S_{p}}^{\mathrm{Pl}_{p}-S_{p}}=\mu_{\mathrm{Pl}_{p}-S_{p}}^{S_{p}}=\mu_{\mathrm{Pl}_{p}-S_{p}},
$$

ainsi que les mêmes égalités pour $r$.

(ii) Les hypothèses permettent d'utiliser la preuve du théorème 13 de [Jaulent et Maire 2003] sans changement.

\section{Remerciements}

Je remercie le rapporteur pour avoir pris le temps de lire et de commenter cet article, en particulier pour m'avoir signalé qu'un résultat de Howson donnait une preuve plus succincte de la première partie du théorème 2.1. Merci aussi à John Coates pour m'avoir suggéré d'étendre mes résultats sur les groupes uniformes aux groupes $p$-valués. J'exprime aussi ma gratitude envers mon directeur de thèse, 
Christian Maire, pour m'avoir fait découvrir un pan très intéressant des mathématiques, ainsi que pour son aide précieuse.

\section{Bibliographie}

[Balister et Howson 1997] P. N. Balister et S. Howson, "Note on Nakayama's lemma for compact $\Lambda$-modules”, Asian J. Math. 1:2 (1997), 224-229. MR 99f:16047 Zbl 0904.16019

[Brumer 1966] A. Brumer, "Pseudocompact algebras, profinite groups and class formations", $J$. Algebra 4 (1966), 442-470. MR 34 \#2650 Zbl 0146.04702

[Coates et al. 2003] J. Coates, P. Schneider et R. Sujatha, "Modules over Iwasawa algebras", J. Inst. Math. Jussieu 2:1 (2003), 73-108. MR 2004b:11152 Zbl 1061.11060

[Cuoco et Monsky 1981] A. A. Cuoco et P. Monsky, "Class numbers in $\mathbf{Z}_{p}^{d}$-extensions", Math. Ann. 255:2 (1981), 235-258. MR 82h:12010 Zbl 0437.12003

[Dixon et al. 1999] J. D. Dixon, M. P. F. du Sautoy, A. Mann et D. Segal, Analytic pro-p groups, $2^{\mathrm{e}}$ éd., Cambridge Studies in Adv. Math. 61, Cambridge University Press, 1999. MR 2000m:20039 Zbl 0934.20001

[Gabriel 1962] P. Gabriel, "Des catégories abéliennes”, Bull. Soc. Math. France 90 (1962), 323 448. MR 38 \#1144 Zbl 0201.35602

[Gras 1998] G. Gras, “Théorèmes de réflexion”, J. Théor. Nombres Bordeaux 10:2 (1998), 399-499. MR 2002g:11154 Zbl 0949.11058

[Gras 2003] G. Gras, Class field theory: From theory to practice, Springer, Berlin, 2003. MR 2003j: 11138 Zbl 1019.11032

[Hachimori et Sharifi 2005] Y. Hachimori et R. T. Sharifi, "On the failure of pseudo-nullity of Iwasawa modules”, J. Algebraic Geom. 14:3 (2005), 567-591. MR 2006a:11138 Zbl 1085.11054

[Harris 1979] M. Harris, " $p$-adic representations arising from descent on abelian varieties", Compositio Math. 39:2 (1979), 177-245. MR 80j:14035 Zbl 0417.14034

[Harris 2000] M. Harris, "Correction to: " $p$-adic representations arising from descent on abelian varieties" [Compositio Math. 39 (1979), no. 2, 177-245; MR0546966 (80j:14035)]”, Compositio Math. 121:1 (2000), 105-108. MR 2001b:11050

[Howson 2002] S. Howson, "Euler characteristics as invariants of Iwasawa modules", Proc. London Math. Soc. (3) 85:3 (2002), 634-658. MR 2004c:11202 Zbl 1036.11053

[Jannsen 1989] U. Jannsen, "Iwasawa modules up to isomorphism", pp. 171-207 dans Algebraic number theory, édité par J. Coates et al., Adv. Stud. Pure Math. 17, Academic Press, Boston, MA, 1989. MR 93c:11095 Zbl 0732.11061

[Jaulent 1986] J.-F. Jaulent, L'arithmétique des l-extensions, thèse d'État, Université de FrancheComté Faculté des Sciences, Besançon, 1986. MR 88j:11080 Zbl 0601.12002

[Jaulent 1998] J.-F. Jaulent, "Théorie $l$-adique globale du corps de classes", J. Théor. Nombres Bordeaux 10:2 (1998), 355-397. MR 2002c:11152 Zbl 0938.11052

[Jaulent 2005] J.-F. Jaulent, "Généralisation d'un théorème d'Iwasawa”, J. Théor. Nombres Bordeaux 17:2 (2005), 527-553. MR 2007e:11132 Zbl 1176.11052

[Jaulent et Maire 2003] J.-F. Jaulent et C. Maire, "Sur les invariants d'Iwasawa des tours cyclotomiques", Canad. Math. Bull. 46:2 (2003), 178-190. MR 2004f:11118 Zbl 1155.11353 
[Lazard 1965] M. Lazard, "Groupes analytiques p-adiques", Inst. Hautes Études Sci. Publ. Math. 26 (1965), 389-603. MR 35 \#188 Zbl 0139.02302

[Lesieur et Croisot 1959] L. Lesieur et R. Croisot, "Sur les anneaux premiers noethériens à gauche", Ann. Sci. École Norm. Sup. (3) 76 (1959), 161-183. MR 22 \#54 Zbl 0092.03802

[Maire 2005] C. Maire, "Sur la dimension cohomologique des pro- $p$-extensions des corps de nombres”, J. Théor. Nombres Bordeaux 17:2 (2005), 575-606. MR 2007b:11179

[Maire 2011] C. Maire, "Plongements locaux et extensions de corps de nombres", Int. J. Number Theory 7:3 (2011), 721-738. MR 2805577 Zbl 05913797

[Nguyen-Quang-Do 1984] T. Nguyen-Quang-Do, "Formations de classes et modules d'Iwasawa", pp. 167-185 dans Number theory (Noordwijkerhout, 1983), édité par H. Jager, Lecture Notes in Math. 1068, Springer, Berlin, 1984. MR 85j:11156

[Ribes et Zalesskii 2000] L. Ribes et P. Zalesskii, Profinite groups, Ergebnisse der Mathematik (3) 40, Springer, Berlin, 2000. MR 2001k:20060 Zbl 0949.20017

[Serre 1966] J.-P. Serre, "Classes des corps cyclotomiques (d'après K. Iwasawa)", Exp. No. 174 dans Séminaire Bourbaki, 1958/1959, W. A. Benjamin, Amsterdam, 1966. Reimpression: pp. 8393 dans Séminaire Bourbaki 5, Soc. Math. France, Paris, 1995. MR 0197243

[Serre 1972] J.-P. Serre, "Propriétés galoisiennes des points d'ordre fini des courbes elliptiques", Invent. Math. 15:4 (1972), 259-331. MR 52 \#8126 Zbl 0235.14012

[Venjakob 2002] O. Venjakob, "On the structure theory of the Iwasawa algebra of a $p$-adic Lie group”, J. Eur. Math. Soc. (JEMS) 4:3 (2002), 271-311. MR 2004h:16029 Zbl 1049.16016

[Washington 1997] L. C. Washington, Introduction to cyclotomic fields, $2^{\mathrm{e}}$ éd., Graduate Texts in Mathematics 83, Springer, New York, 1997. MR 97h:11130 Zbl 0966.11047

Communicated by John H. Coates

Received 2010-07-19 Revised 2011-02-25 Accepted 2011-05-08

guillaume.perbet@univ-fcomte.fr Laboratoire de Mathématiques, UMR 6623, UFR Sciences et Techniques, 16 route de Gray, 25030 Besançon, France 


\section{Guidelines for Authors}

Authors may submit manuscripts in PDF format on-line at the Submission page at the ANT website.

Originality. Submission of a manuscript acknowledges that the manuscript is original and and is not, in whole or in part, published or under consideration for publication elsewhere. It is understood also that the manuscript will not be submitted elsewhere while under consideration for publication in this journal.

Language. Articles in ANT are usually in English, but articles written in other languages are welcome.

Required items. A brief abstract of about 150 words or less must be included. It should be self-contained and not make any reference to the bibliography. If the article is not in English, two versions of the abstract must be included, one in the language of the article and one in English. Also required are keywords and subject classifications for the article, and, for each author, postal address, affiliation (if appropriate), and email address.

Format. Authors are encouraged to use LTEX but submissions in other varieties of $\mathrm{T}_{\mathrm{E}} \mathrm{X}$, and exceptionally in other formats, are acceptable. Initial uploads should be in PDF format; after the refereeing process we will ask you to submit all source material.

References. Bibliographical references should be complete, including article titles and page ranges. All references in the bibliography should be cited in the text. The use of BibTEX is preferred but not required. Tags will be converted to the house format, however, for submission you may use the format of your choice. Links will be provided to all literature with known web locations and authors are encouraged to provide their own links in addition to those supplied in the editorial process.

Figures. Figures must be of publication quality. After acceptance, you will need to submit the original source files in vector graphics format for all diagrams in your manuscript: vector EPS or vector PDF files are the most useful.

Most drawing and graphing packages (Mathematica, Adobe Illustrator, Corel Draw, MATLAB, etc.) allow the user to save files in one of these formats. Make sure that what you are saving is vector graphics and not a bitmap. If you need help, please write to graphics@msp.org with details about how your graphics were generated.

White space. Forced line breaks or page breaks should not be inserted in the document. There is no point in your trying to optimize line and page breaks in the original manuscript. The manuscript will be reformatted to use the journal's preferred fonts and layout.

Proofs. Page proofs will be made available to authors (or to the designated corresponding author) at a Web site in PDF format. Failure to acknowledge the receipt of proofs or to return corrections within the requested deadline may cause publication to be postponed. 


\section{Algebra \& Number Theory}

Volume $5 \quad$ No. $6 \quad 2011$

Higher direct images of the structure sheaf in positive characteristic

ANDRE CHATZISTAMATIOU and KAY RÜLLING

Geometry of quiver Grassmannians of Kronecker type and applications to cluster algebras

Giovanni Cerulli Irelli and Francesco Esposito

Sur le groupe de Chow de codimension deux des variétés sur les corps finis

ALENA PIRUTKA

Sur les invariants d'Iwasawa dans les extensions de Lie $p$-adiques

Guillaume Perbet 